This item was submitted to Loughborough's Research Repository by the author.

Items in Figshare are protected by copyright, with all rights reserved, unless otherwise indicated.

\title{
The behaviour of optimal Lyapunov functions
}

PLEASE CITE THE PUBLISHED VERSION

PUBLISHER

(C) D.N. Shields

\section{PUBLISHER STATEMENT}

This work is made available according to the conditions of the Creative Commons Attribution-NonCommercialNoDerivatives 4.0 International (CC BY-NC-ND 4.0) licence. Full details of this licence are available at: https://creativecommons.org/licenses/by-nc-nd/4.0/

\section{LICENCE}

CC BY-NC-ND 4.0

REPOSITORY RECORD

Shields, Derek N.. 2019. “The Behaviour of Optimal Lyapunov Functions”. figshare. https://hdl.handle.net/2134/35647. 


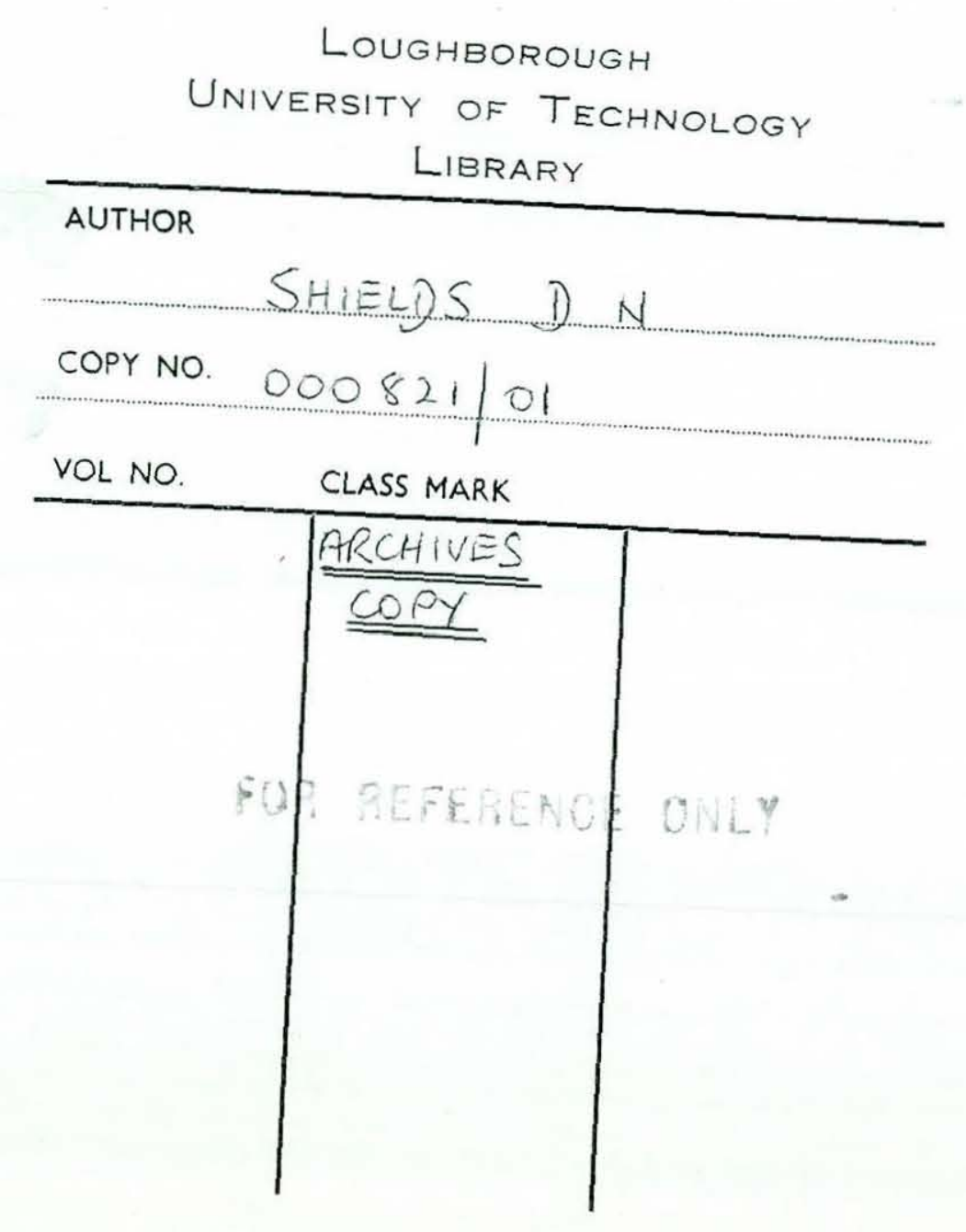





\section{TYE BEYAVIOUR OF OPTIMAI}

\section{IYYAPUNOV INUNCTIONS}

Derek Nicholas Shields BSc., D.I.S. (Loughborough)

A Doctoral Thesis

Submitted in partial fulfilment of the requirements for the award of Doctor of Philosophy of the Loughborough University of Technology.

October 1973

Supervisor: Prof. C. Storey,

Department of Mathematics.

(C) by D.N. Shields, 1973 


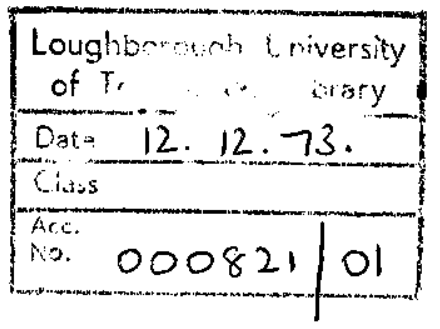




\section{Summary}

The use of Lyapunov's direct method in obtaining regions of asymtotic stability of non-linear autonomous systems is well known. This thesis is an investigation into the optimization of some function of these systems over different classes of Lyapunov functions.

In Chapter 2 bounds on the transient response of two systems are optinized over a subset of quadratic Lyapunov functions and numerical work is carried out to compare several bounds.

Zubov's equation is the subject of Chapter 3. The nonuniformity of the series-construction procedure is studied analytically and a new approach is made to the solution of the equation by finite difference methods.

Chapters 4,5 and 6 have a common theme of optimizing the RAS over a class of Lyapunov functions. Chapter 4 is restricted to optimal quadratics which are investigated analytically and numerically, two algorithms being developed. An optimal quadratic algorithm and a RAS algorithm are proposed in Chapter 5 for high order systems. Extensions are made in Chapter 6 to optimal Lyapunov functions of general degree and relay control systems and systems of Lure' form are considered. 


\section{Acknowledoments}

The author vould like to thank Professor C. Storey for his guidance and encouragement in carrying out this work. His thanks are also due to the efficient work of the LUT computing centre and his wife, Greta, for typing this thesis. 


\section{Contents}

Chapter 1.: Introduction

page

1.1 Discussion

1.2 Preliminaries 2

1.3 Basic Definitions of Stability 4

1.4 The Autonomous Case 6

1.5 The Theorems of Lyapunov, 7

1.6 A Practical RAS For The Autonomous

Case

1.7 Motivation

11

1.8 Contents of Chapters and Background Material

Chapter 2: Optimal Bounds on the Response of

Non-linear Stab1e Systems

2.1 Introduction

2.2 The Optimum $\eta$

2.3 The Minimum Condition Number, $\mu(P) \quad 24$

2.4 A Conjecture of Wiberg

2.5 An Optimum Class of Matrices

2.6 Bounds on $\mu(P) \quad 28$

2.7 Non-uniqueness of $P$ in Form 2.2.2 30

2.8 Numerical Optimum of the Condition Number

2.9 C in Jordan Form

2.10 Somo Numerical Experiments

Chapter 3 : The Determination of Stability

Regions by Zubov's Approach

3.1. Introduction

3.2 The Main Theorem

3.3 The Construction Procedure 
3.4 An Example (Zubov)

3.5 Direct Numerical Solution of The PDE 47

3.6 Numerical Examples $\quad 50$

3.7 Recent Methods 52

Chapter 4 : Optima1 Quadratic Lyapunov. Functions

4.1 Introduction $\quad 58$

4.2 Examples on the Determination of :60

Optimal Quadratics and Simple RAS's

4.3 Numerical Determination of a RAS

and an Optimal Quadratic 72

4.4 Numerical Results 77

4.5 Optimal Quadratics for a Restricted

Class of High Order systems 82

4.6. Some Numerical Results

Chapter 5: Computational Methods for Optimal

Quadratic and RAS Determination for

General Non-linear Systems

5.1 Introduction

104

5.2 An Optimal Quadratic Algorithm "

5.3 Numerical Results , 113

5.4 A Method for Quadratic RAS

Determination for High Order

Systems

Chapter 6 : General Optimal Lyapunov Functions

for Non-linear Systems Including

Those of Lure' Form and Relay

Control Systems

6.1. High Degree Lyapunov Functions for

Autonomous Non-linear Systems $\quad 130$

Numerical Examples 133 
6.2 Optimum Lyapunov Functions for

page

Relay Contro1 Systems $\quad 139$

Numerical Examples $\quad 142$

The Piecewise Linear LF. $\quad 144$

6.3 Finite Regions of Attraction for

the Problem of Lure' 148

An Optimal Quadratic for the

Infinite Sector. 149

Variation of $\rho$ with $q \quad 152$

Conclusions

164

References

170

Appendix 1

176

Appendix 2

177

Appendix 3

179

Appendix 4

132

Appendix. 5

185 


\section{CHAPTER 1}

INITRODUCTION 


\section{Chas ter 1}

\section{Introduction}

\subsection{Discussion}

The classical idea of stability originated in the motion of rigid bodies in mechanics. An equilibrium was said to be stable if a body returned to its original position after a small displacement. In the last twenty years this idea of stability has been extended considerably both in depth and scope, and powerful tools now exist to treat the stability of a large number of dynamic motions or systems. The most striking development has been the direct method of Lyapurov with its many theoretical and applicative aspects. This thesis is mainly concerned with 'optimum problems' in the use of soicalled Iyapunor functions to find estimates of transient response and of the domain of attraction of nonlinear autonomous differential equations. 


\subsection{Preliminaries}

In what follows the usual notations for vectors and matrices in n-dimensional Euclidean space will apply throughout (See $(14))$.

Elements of $E^{n}$ will be denoted by $x, y$ etc and will be treated as column vectors, although written as rows in long hand i.e.

$$
\underline{x}=\left(x_{1}, x_{2}, \ldots \ldots, x_{n}\right)
$$

$\|\cdot\|$ is the Euclidean norm defined as

$$
\|\underline{x}\|^{2}=\underline{x}^{T} \underline{x}=\sum_{i=1}^{n}\left|x_{i}\right|^{2}
$$

The elements of matrices $A, B$ etc. will be denoted by $a_{i, j}$ and $b_{i, j}$ respectively.

$E^{n}$ may also be called the statespace or phase space depending upon the nature of $x$. For $n=2$ we may write $x=(x, y)$.

We will be concerned with the vector differential equation

$$
\dot{x}=\frac{d x}{d t}=\underline{e}(\underline{x}, t)
$$

where $x \in E^{n} ; t$ is an independent parameter, usually the time; and $f \in E^{n}$, whose components $f_{i}(x, t)$ are functions of $x_{i}$ and $t$ (specifically $\underline{f}$ is the map f: $E^{n} \times R \rightarrow E^{n}$, $R$ the real Iine). 
The form of 1.1 .1 is quite general and it can be consiajered as a systern of $n$ pirst order equations. The important nth order scalar equation,

$$
y^{(n)}= \pm\left(y^{(n-1)}, y^{(n-2)}, \cdots, y^{(1)}, y, t\right)
$$

where

$$
\mathrm{y}^{(n)}=\frac{\mathrm{d}^{\mathrm{n}} \mathrm{y}}{\mathrm{dt} \mathrm{t}^{\mathrm{n}}} \quad 1.2 .1
$$

is reducible to this form by defining

$$
\begin{aligned}
& x_{1}=y \\
& \dot{x}_{1}=x_{2} \\
& \dot{x}_{2}=x_{3} \\
& \quad \cdot \dot{x}_{n-1}=x_{n} \\
& \left.\dot{x}_{n}=x_{\left(x_{n}\right.}, x_{n-1}, \ldots, x_{1}, t\right)
\end{aligned}
$$

If $\underline{f}$ in 1.1 .1 is independent of $t$ we have

$$
\underline{\dot{x}}=\underline{\underline{x}}(\underline{x})
$$

an autonornous systen with special properties (Șee later or Zubov (1)).

If the right hand side of 1.1 .1 is continuous and the existence and uniqueness of solutions is assured together with their continuous dependence on initial values, $f$ rill be said to be of class $\mathrm{E}, \pm \in E$. Let $\left(\underline{x}_{0}, t_{0}\right)$ be the initial values and let $\underline{f} \in \mathbb{E}$, then define $z\left(t, \underline{x}_{Q}, t_{0}\right)$ as the solution of 1.1 .1 , i.e. $\frac{d z}{d t}\left(t, \underline{x}_{0}, t_{0}\right)=\underline{E}\left(\underline{z}\left(t, \underline{x}_{0}, t_{0}\right), t\right) \quad 1.2 .4$.

with $z\left(t_{0}, x_{0}, t_{0}\right)=\underline{x}_{0}$

The singular or equilibrium points of 1.1 .1 are the constant solutions $Z\left(t, \underline{x}_{0}, t_{0}\right)=\underline{x_{0}}$ or, equivalently, the solutions $\underline{x}$ satisfying $\underline{\underline{x}}(\underline{x}, \dot{t})=0$. 
By simple transition of co-ordinates any singular point may be brought to the origin, $\underline{x}=0$. Henceforth, we assume $\underline{f}(\underline{o}, t)=0$ and that the origin is an isolated singular point (i.e. no other such point exists in a neighbourhood of $x=0$ ). Define the $(n+1)$ - dimensional space of quantities $(x, t)$ as the motion space, then a motion of 1.1 .1 is the continuous path formea by the set $\left(\underline{z}\left(t, \underline{x}_{0}, t_{0}\right), t\right)$. A trajectory is the projection of this path onto the phase space and a half trajectory is a trajectory defined for some $t \geqq t_{0}$ ( or $t \leqq t_{0}$ ).

1.3. Basic Definitions of Stability in the Sense of Lyapunov

Denote by $\mathrm{R}(\mathrm{h})$ the region $\mathrm{R}(\mathrm{h}):\{\underline{x} /\|\mathrm{x}\| \leq \mathrm{h}\}$, or more generally, let the set $\left\{(x, t) /\|\underline{x}\| \leq h, t \geq t_{0}\right\}$ be denoted by $R\left(h, t_{0}\right)$. Suppose in $R\left(h, t_{0}\right)$

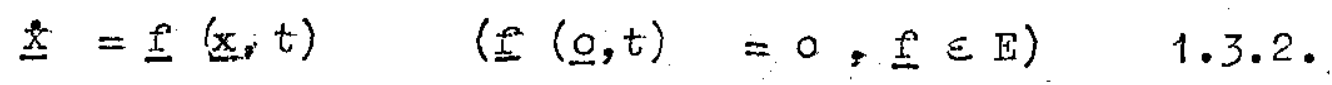

Then two definitions are basic to Lyapunov's direct method: Def. 1.3 .1

The origin of the differential equation ( $d_{0}$ e.) 1.3.2. is said to be stable if there exists for any $\epsilon>0$ a number Srosuch that.

$\| \underline{x}$ l $<\delta$

implies

$\left\|\underline{Z}\left(t, \underline{x}_{0}, t_{0}\right)\right\|<\epsilon, \forall t \equiv t_{0}$ 
Def. 1.3.2

The origin of the d.e. 1.3.2 is said to be asymptotically stable (a.s.) if it is stable and there exists a. $\delta_{0}$ such that for

follows

$$
\|\underline{x}\| \| \delta_{0}, \delta_{0}>0
$$

$$
\lim _{t \rightarrow \infty}\left(t, \underline{x}_{0}, t_{0}\right)=0
$$

If $1 \cdot 3.3$ holds for all $\underline{x}_{0} \in E^{n}$ in Def. 1.3.2 the origin is said to be a.s. in the whole. Further definitions are given in Lefshetz (14), Zubov (1) etc., including instability definitions. Good critical treatments of these and other definitions are given in Hahn (15) and Lehnigk (17).

Finally, the domain of attraction (DOA) of an a.s. system 1.3 .2 is the set $U$ defined by $U\left(t_{0}\right)=\left\{\underline{x}_{0} / \operatorname{Iim}_{t \rightarrow \infty}\left(t, \underline{x}_{0}, t_{0}\right)=0\right\} 1.3 .4$. 
For the autonomous case $U$ is indepencient of $t_{0}$.

1.4. The Autoromous Case

The main systen in the following chapters is system 1.2 .3 namely,

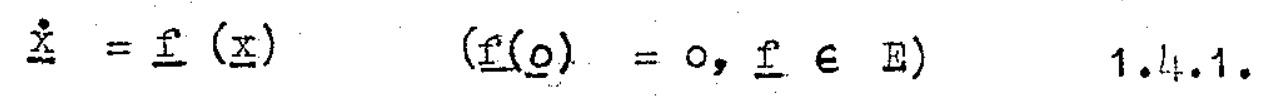

Two forms of 1.4.1, one particular and one Eeneral, are the following:

a) the Iinear system.

$$
\dot{x}=A x \quad 1.4 .2
$$

where $A$ is an $n x n$ matrix wich is said to be stable if its eigenvalues, $\lambda_{i}, 1=1, n$, have negative real parts. The system is callea significant if $\operatorname{Re}\left(\lambda_{j}\right) \neq 0$

b) The system

$$
\ddot{x}=A \underline{x}+g(\underline{x})=\underline{I}(\underline{x})
$$$$
1.4 .3 .
$$

where $g(x)$ possesses a convergent power series expansion about the origin whose terms are of degree wo and greater. Here, $A \underline{x}$ is calied the first approximation or Iinear part of 1.4.3. A can be regarded as the Jacobian of $f$ at $x=0$,

$$
A=\frac{\partial f(\underline{x})}{\partial \underline{x}} \mid \underline{x}=0 .=\left\{\left.\frac{\partial f_{i}^{i}}{\partial x_{j}^{j}}\right|_{x=0}\right\} 1.4 .4 .
$$




\subsection{The Theorems of Lyapunov}

The second method of Lyapunov attempts to determine the stability of the equilibrium without prior knoitledge of the solutions of differential equations. It introduces the idea of a certain function called a Iyapunov function which possesses properties analogous to those of the total energy of a dissipative dynamic system. The energy in the latter is positive and non-increasing near a stable equilibrium. Formally, let $V(x)$ be a continuous scalar function defined in some region $R(h)$ and possessing continuous first partial derivatives. Then the following definitions and theorems are pertinent to the autonomous system 1.4.1 (See Hahn (15) for general case of system 1.3.2)

Def. 1.5 .1$.

The function $V(\underline{x})$ is positive (negative) definite if in some region $R\left(h_{1}\right)$,

$$
\begin{aligned}
& V(\underline{x})>0 \cdot(<0) \text { and } V(\underline{0})=0 . \text { If } \\
& V(\underline{x}) \Rightarrow 0 \quad(\underline{0}) \text { and } V(\underline{0})=0 . \text { it is }
\end{aligned}
$$

positive (negative) semi-derinite.

$V(x)$ is called strictly nositive definite if

$V(\underline{x})>0$ for $\underline{x} \in \mathbb{E}^{n}, \underline{x} \neq 0$; ard radially

unibounded if " $\|$ In $\rightarrow \infty$ implies. $V(x) \rightarrow \infty$. If in sone $R(h), V(x)$ is positive definite and its total derivative $\dot{V}$, where $\dot{V}=Z V^{\top} \underline{\underline{I}}(\underline{x})=\sum_{i=1}^{n} \frac{\partial v}{\partial x_{i}} f_{i}(\underline{x})$, 
is negative semi-definite, it is called a Lyapunov function (LT) for system 1.1.1.

\section{Theorem 1.5.1.}

The equilibrium of system 1.4.1. is

(a) stable if there exists a Lyapunov function and

(b) a.s. If $V(\underline{x})$ is positive definite and $\dot{V}$ is

negative definite with respect to 1.4 .1 .

Theoren 1.5.2. (Barbasin (18))

The equilibriurn of 1.4 .1 is a.s. if

(a) $V(x)$ is positive definite and

(b) $\dot{V}$ is negative semi-definite and does not vanish identically on any non-trivial trajectory of 1.4 .1 . These two theorems are purely local in character and give little information as to the size of the actual stability regions. In this respect the folloming theorem is of great practical importance. Theorem 1.5.3. (Lefshetz (14))

Trajectories of 1.4 .1 which start "from a region

$D$ containing the origin will be a...*. if there exists

a function $V(\underline{x})$ with the properties:

(a) $v(x)$ is positive def. in $D$,

(b) $\dot{V}$ with respect to system 1.4 .1 is at least ner. semi-definite,

(c) $\dot{V}(x) \neq 0$ on anj trajectory of 1.4 .1 in $D$ excent $x=0$,

* (An a.s. trajectory is one originating from some initial $\underline{x}_{0} \in D$, an RAS ) 
(d) $\nabla V(x) \neq 0$ in D except for $x=0$,

(e) one of the level surfaces $V=$ constant bounds $D$.

Let $V(\underline{x})=c$ be a level surface bounding a region $D$,

$D: \quad\{\underline{x} / V(\underline{x})<c, c=0\}$

Henceforth, the recion $I_{\max }$ bounded by the surface $V(\underline{x})=c_{\max }$, where $c_{\max }$ denotes the largest $c$ for which proporties (a) to (e) hold, will be called the region of asmmtotically stability (RAS) of the Iytounov function $V(x)$ for the system 1.4 .1 . If this RAS is untounded then 1.4 .1 is a.s. in the whole.

From Theorem $A 1.1$ we see that the level $V(x)$ surfaces will be closed and hence bounded in some neighbour cood of the origin.

Theorem 1.5.L. (Linear Case)

The linear systern 1.4 .2 is a.s. Iff $A$ is a stability matrix.

Theorern 1.5 .5 .

The origin of 1.4 .2 is a.s. iff there exists a positive definite symetric matrix $\mathrm{P}$ winch is the unique solution of the Iyapunov matrix equation.

$A^{P} P+P A=-Q$ 1.5 .1

for any positive definite symnetric $Q$. (The theorem also holds with $P$ and $Q$ p.d. hermitian matrices). 
Theorem 1.5.6

If the stability of the first approximation of 1.4 .3

is significant then the stability behaviour of the linear part (1.4.2) and the complete system (1.4.3) are the same.

For less restrictive conditions on $g(\underline{x})$ in 1.4 .3 . see Hahn (15) or Lehnigk (17): A comprehensive treatment of the matrix equation 1.5 .1 is given by Bannett (20).

1.6. A Pracitical DAS For the Autonomous Case Let $A$ be a stability matrix in systen 1.4 .3 . Then a general class of Iyapunov functions for this syten can be generated as follows. Let $V(x)$ be of the form

$$
V(x)=\underline{x}^{\top} \mathrm{P} \underline{x}+V_{0}(\underline{x}) \quad 1.6 .1 \text {. }
$$

where $P$ is a unique solution of 1. 5.1. for some positive definite $Q$ and where $V_{0}(x)$ can be exparded. as a Taylor series with terms of degree three and greater. Sirce

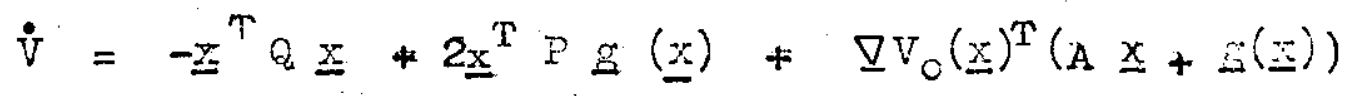

$$
1.6 .2
$$

by the ascumptions on $E(x)$ and $V_{0}$ and the properties of quadratic forms, $f<0$ in $R(h)$ for $z \neq 0$ and $h$ snall. We have

Theoren 1.6.1

Let $\mathrm{E}_{\mathrm{V}}$ be the set

$$
\left.E_{\mathrm{V}}:\{\underline{X} / \underline{X})=0, X \neq 0\right\} \quad 1.6 .3
$$

Then the RAS indicated by the Iyapunov function (II) $V(\underline{x})$ of 1.6 .1 is siven by $D$ where

$$
D:\{x / V(x)<0 \min \} \quad 1.6 . \therefore
$$


and where

$$
\mathrm{C}_{\min }=\operatorname{minV}(\underline{\mathrm{x}}), \underline{x} \in \mathrm{I}_{\mathrm{v}} \quad 1.6 .4
$$

Appendix 1 (A1) gives some useful definitions on the closed contours of Lyapunov functions.

\subsection{Motivation}

Two main probleris are inherent in using Lyapunov methods to find regions of a.s. of autonomous differential equations,

(a) the construction of a suitable LF,

(b) the determination of the RAS indicated by that LF. Many methods exist to solve (a) (see Tait (37) and

- Brockett (38) for bibliographies). Some were developed for a specific form of differential equation, others were more general. Hewit (2) has developed computer algorithms for their construction and compared their RAS's. The main notivation of this thesis has been that whereas a great deal of research has centred on constructing LF's little attention has been paid to finding the 'best' LF of a given class. Here, 'best' need not be interpreted solely in terms of the RAS but also in terms of the transient response or some other function of the system. Emphasis has therefore been placed on finding 'optimum results' where possible and in showing what properties if any, these 'optimal Lyapunov functions' possess.

Some attention has been centred on finding analytic results for simple specific cases, which have given a lead to the dovelopment of numerical algorithms needed to study more complex cases. 
1.8 Contents of Chapters and Background Material

Many authors, including Kalman and Bertram (16),

Vogt (19), Zubov (1) and Wiberg (23), have used the fact that if $V(\underline{x})$ is a Lyapunov function for the autonomous system 1.4 .3 (or the more general system 1.3 .2 ) giving asymptotic stability, then minimizing the expression $(-\dot{V} / v)$,

$$
\alpha=\min _{x \in R(h)}(-\dot{V} / V)
$$

for some sufficiently small region $R(h)$, implies the inequality

$$
v(\underline{x}(t)) \leq e^{-\alpha t} v(\underline{x}(0))
$$

The quantity $\alpha^{-1}$ may be interpreted as the largest timeconstant over the region $R(h)$ of the phase space and is therefore a figure of merit of the system.

In Chapter 2 we extend some work of Wiberg (23) and maximize $\alpha$ over a sub-class of quadratic Lyapunov functions (with given $R(h)$ ) for the system 1.4 .3 and the more general system

$$
\underline{x}=A \underline{x}+G(\underline{x}, t) \underline{x}+\underline{u}(t) \quad \text { (A stable) }
$$

An optimizing condition is found when A is in companion form (CF) with real eigenvalues and some useful bounds are proposed, the latter being tested by numerical work. Some numerical work is also conducted in determining whether some bounds of Vogt (19) are useful in locating the real parts of the eigenvalues of $A$.

In Chapter 3 we consider Zubov's (1) partial differential equation (PDE) for the autonomous system 1.4.3,

$$
\sum_{i=1}^{n} \frac{\partial V}{\partial x_{i}} f_{i}=-\phi(1-v)
$$

A wealth of numerical experience on the application of its 
solution by the series procedure (mainly by Hewit (2), Margolis and Vogt (4), Rao and De Sarker (5), and $\mathrm{Yu}$ and Vongsuriya (6)) has shown that the RAS's of the high degree LF's are often inferior to those of lower degree. No analytic study of this non-uniformity has yet been attempted.

In the first part of this chapter a system showing this non-uniformity is investigated analytically and an important question emerges concerning the raggion of convergence of the series-l.yapunov function. In the second part we look at an alternative way of solvine the PDE by finite-difference methods. By using polar co-ordinates an initial value problem results and solution by a CrankNicholson-type difference scheme is possible. Numerical examples show that the solution breaks down near the DOA boundary. Some other methods are also considered.

Chapters 4,5 and 6 have a common thene in that for the system of the form 1.4 .3 , and also for relay systems in chapter 6, the problem of maximizing the RAS over a class of Lyapunov functions is considered. The stated problem is

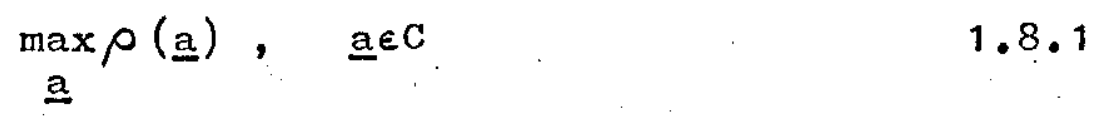$$
\max \rho(\underline{a}), \quad \underline{a} \in \mathrm{C}
$$$$
\text { a }
$$

where $C$ is a parameter class determining the LF and $\rho$ (a) is a measure of the size of the RAS

$$
\mathrm{v}(\underline{\mathrm{x}}, \underline{\mathrm{a}})<\mathrm{v}_{\mathrm{m}}(\underline{\mathrm{a}}) \quad \mathbf{1 . 8} .2
$$

where

$$
\mathrm{V}_{\mathrm{m}}(\underline{\mathrm{a}})=\min _{\underline{x}} \mathrm{~V}(\underline{\mathrm{x}}, \underline{\mathrm{a}})
$$


subject to

$$
\dot{\mathrm{v}}(\underline{\mathrm{x}}, \underline{a})=0, \underline{x} \neq 0 \quad 1.8 .4
$$

The main difficulty is the RAS determination of finding $V_{m}$ in 1.8.2. Research on the subject is divided into two main camps of either treating 1.8 .3 analytically as an equality-constrained optimization problem, or geometrically, as a tangency between two hypersurfaces, $\mathrm{V}(\underline{\mathrm{x}}, \underline{\mathrm{a}})=\mathrm{V}_{\mathrm{m}}(\underline{\mathrm{a}})$ and $\dot{\mathrm{V}}(\underline{\mathrm{x}}, \underline{\mathrm{a}})=0$. Rodden (3) gave a method for the latter which Hewit (2) has improved and applied to some second order systems. Hewit (2) has used the method to optimize the average radius of the RAS for a number of LF's determined by his construction procedures for the methods of Zubov, Szegö, Ingwerson and Krasovski.

Advocates of the equality-optimization approach have. been in the main Szegö (52), Geiss $(50,51)$, Julich (57), Muddle (58) and Lapidus and Berger (49). They all use the penalty function method. For instance, Geiss, Julich and Szégö use either

$$
F_{1:}=v+\pi \dot{v}^{2} /\|x\|^{2}
$$

or

$$
F_{2}=v+K \dot{V}^{2}
$$

and for increasing $K$ minimize $F_{1}$ or $F_{2}$ via some powerful minimization technique such as Davidon-Fletcher-Powell (60), thus reducing 1.8 .3 to a sequence of unconstrained minimizations which hopefully converge to the constrained minima (Fiacco and McCormick (59) discuss convergence). Once $V$ has been chosen therefore, the problem is taken out of the realm of Lyapunov theory and into that of non-linear optimization where new powerful algorithms can be applied. Variations 
are the choice of penalty function and the minimization method.

In Chapter 4 the class of Lyapunov functions is that of quadratics, $V=\underline{x}^{T}{ }_{x}$, with $P$ determined through the matrix equation 1.5 .1 for $Q$ p.d. Due to the complexity of the RAS determination, analytic results in aplying problem 1.8.1 to various systems have been scarce. Geiss (50) found an optimal quadratic for a second order Duffing equation but the example gives little insight into the nature of the problem. It is surprising that the numerical algorithms developed so far for maxinizing $\rho(\underline{a})$ for a given $L F$ are based on 1ittle understanding of the relationship between the optima1 RAS boundary, $V=V_{m}$ say, and its constraint contour, $\dot{v}=0$. (Wilson (61) gives a topological account of the V-contours but the constraint contour is not investi. gated). In Chapter 4 five systems are studied analytically as far as possible through the Lagrange equations. Although only two optimal quadratics are obtained, sufficient insight is gained on which to base a numerical investigation. An efficient and accurate algorithm is developed for RAS determination for a restricted class of second order systems, and optimal quadratics are found via Powell's (33) conjugate gradient algorithm for a number of systems. Extension to higher order systems is made. The analytic and confirming numerical results exhibit an 'equal tangency property', namely, that for many systems a subset of quadratics exist such that their RAS boundaries have at least two points of contact (not radially symmetric) with their constraint contours, $\dot{v}(\underline{x})=0$.

In Chapter 5 two algorithms are proposed. The first is an optimal quadratic algorithm based on an idea of 
Davidson and Kurak (47) who reduce the optimum quadratic problem to one of a constrained optimization which they solve via use of Rosenbrock's (32) method. The proposed algorithm takes into account the work of Chapters 2 and 4 and replaces Rosenbrock's hill-climber by a variation of the Complex method of Box (55). A comparison is made between the two methods for 2 second order and 4 third order systems, showing the proposed method is superior.

The second algorithm incorporates some features of the previous algorithm and determines the precise RAS for a quadratic LF via a special penalty function of Miele (62) (reference is made to one of the four authors) which is minimized by the Fletcher Powel1 (60) method of conjugate directions: The drawback of many penalty function methods noteably those of Julich (57) and Lapidus (49) - is that no automatic method is proposed to find the 'global' minimum. An exception is that of Geiss (50) who encloses a possible RAS boundary, $\underline{x}^{T}{ }^{T} \underline{x}=V_{m}$, with an $n$-dimensional box inside which $\dot{V}$ is determined at random points. However the method is time consumming. The proposed method finds the 'global' minimum of the penalty function automatically and has good convergence.

Chapter 6 is an extension of Chapter 4 in that the optimal properties of general Lyapunov functions are investigated. The work is divided into three sections which correspond to the three different systems considered; the Eeneral autonomous system 1.4 .3 , a relay control system and a system of Lure' form.

The problem in 1.8 .1 of maximizing $p(\underline{a})$ for a $L F$ of. degree mv 


$$
v=\sum_{i=2}^{m y} v_{i} \quad\left(v_{i} \text { poly. of deg. i }\right) \quad 1.8 .5
$$

has been considered by Szegö (52) and Hewit (2) and others. However, due to the number of independent parameters involved for a LF of degree mv $(2+(m v+5)(m v-2) / 2)$ Iittle numerical experience has resulted, even on comparing their RAS's. Szegö (52) proposed solving the RAS problem of 1.8 .3 and 1.8 .4 via a penalty function approach using the Fletcher-Powell (60) minimization routine. He then maximizes $r$, the distance of the nearest point of the RAS boundaxy, $\mathrm{V}=\mathrm{V}_{\mathrm{m}}$, to the origin, over the co-efficients of the $v_{i}$ terms by Porell's (33) method. Disappointingly, only an optimal quadratic is obtained and that for a simple example where a global search for the minimum in 1.8 .3 is not required. In Chapter 6 the problen is investigated fully via Rodden's (3) method and Nelder and Mead (34) Simplex optimization on $\rho$ (a) (average radius). The 2 mth degree $I F$ of the form

$$
v_{2 m}=\prod_{k=1}^{m} x^{T} P_{k} \underline{x}
$$

is also considered. The optimal RAS's are compared and a multiple tangency phenomenon is exhibited for a number of second order systems.

In the following section we extend some work of Weissenberger $(48,66)$ for the relay system

$$
\underline{x}=A x+\underline{b} \operatorname{sgn} \sigma, \quad \sigma=\underline{a}^{T} \underline{x}
$$

who showed that under certain conditions LF's of the forms:

$$
\text { a) } v=\underline{x}^{T} P \underline{x}+\left|\underline{d}^{T} \underline{x}\right|
$$


and
b) $v=\sum_{i=1}^{m}\left|c_{i}^{T} x\right|$

could be used. A number of second order systems are studied numerically for LF (a) showing 'equal tangency properties'. Using this property an optimum RAS is found analytically for LF (b).

In the final section a connection between the work of Walker and McClamroch (73) and that of Veissenberger (72) is found concerning an optimal quadratic for the Lure' system

$$
\underline{x}=A \underline{x}+\underline{b} \operatorname{sen} \sigma, \quad \sigma=\underline{c}^{T} \underline{x}
$$

where the sector condition

$$
0<f(\sigma) / \sigma<\mathrm{K}
$$

is satisfied only for some region $\sigma_{2} \leqslant \sigma \leqslant \sigma_{1}$. Some extensions are considered.

The computing times given in this thesis are all in terms of mill/secs. (ICL 1905) and serve only as a comparison, all other conditions being equal. All programs were written in FORTRAN 4 and only a listing of the optimal quadratic algorithm of $\mathrm{C} 5^{*}$ is included. Several programs were written using graph plotter routines to trace the required Lyapunov contours, points on which were joined by straight line segments. Diagrams and tables are included in the text for continuity, whereas the figures appear at the back of each chapter and, as far as is convenient, in numbered ordex. 


\section{CHAPTHE 2}

OPTIMAL BOUNDS ON THE RISSPONSE OF

NON-LINEAR STABLE SYSTENS. 


\section{Chapter 2}

\section{Optimal Bounds on The Response of Non-linear Stable Systems.}

\subsection{Introduction}

In the design of a control system it is useful to predict a conservative bound on the response of the system, which takes into account noise and perturbation effects, or to predict a crude approximation to the domain of attraction.

Consider the two systems

$$
\begin{aligned}
& \underline{\dot{x}}=\mathrm{A} \underline{x}+\mathrm{G}(\underline{\mathrm{x}}, t) \underline{\mathrm{x}}+\underline{\mathrm{u}}(t) \quad 2.1 .1 \\
& =\underline{f}(\underline{x}, t) \quad(\underline{f} \in \mathbb{E}) \\
& \underline{\dot{x}}=\mathrm{A} \underline{\mathrm{x}}+\underline{g}(\underline{\mathrm{x}}) \\
& 2 \cdot 1 \cdot 2
\end{aligned}
$$

and

with A stable.

In the former Gx is regarded as the non-linear or perturbation term and $y$ the input to the system; both are assumed to be bounded,

$$
\begin{array}{llll}
\|\mathrm{u}(\mathrm{t})\| \leq \mathrm{c}_{1}, & \forall & \mathrm{t} & 2.1 .3 \\
\|G(x, t)\| \leq \mathrm{c}_{0}, & \forall & t & 2.1 .4
\end{array}
$$

We assume that no real $y$ exists such that $A: G(y, t)=0$ inside some $R(h)$, then the origin wiIl be an isolated singularity. System 2.1 .2 is that of 1.4 .3 .

$$
\begin{aligned}
& \text { For both systems choose the LF } \\
& \quad \mathrm{V}=\underline{x}^{\mathrm{T}} \mathrm{P}
\end{aligned}
$$$$
2.1 \cdot 5
$$

where $p$ solves

$$
A^{\mathrm{T}} \mathrm{P}+\mathrm{PA}=-\mathrm{Q} \quad 2.1 .6
$$

and where $Q$ and hence $P$ magr be general positive definite symmetric matrices. Then for 2.1 .1 


$$
\dot{V}=-\underline{x}^{T} Q \underline{x}+2\left(\underline{x}^{T} G(\underline{x}, t)+\underline{u}^{T}(t)\right) P \underline{x} \quad 2.1 .7
$$

and for 2.1 .2

$$
\dot{\mathrm{v}}=-\underline{\mathrm{x}}^{\mathrm{T}} \mathrm{Qx}+2 \underline{x}^{\mathrm{T}} \mathrm{Pg}(\underline{\mathrm{x}}) \underline{\mathrm{x}} \quad 2.1 .8
$$

Folloring Kalman and Bertram (16) we use several matxix inequalities and the Schwartz inequality to give, respectively

$$
\dot{v} \leq\left(-\pi+2 c_{0} \sqrt{\mu}\right) v+2 c_{1} \sqrt{M(p) v} \quad 2.1 .9
$$

and

$$
\dot{V} \leq(-\pi+2 \mu\|\underline{g}(x)\| /\|x\|) v \quad 2.1 .10
$$

Here $\lambda(A)$ denotes an eigenvalue of $A, M(A)=\max \operatorname{Re} \lambda(A)$ and $m(A)=\min \operatorname{Re} \lambda(A)$. Also

$$
n=\min _{\underline{x} \neq 0 x^{2} Q x}
$$

and

$$
\begin{aligned}
& \quad \mu=\mu(P)=M(P) / m(P) \text {. It can be shown that }(16) \\
& \Pi=m(P-1 Q) .
\end{aligned}
$$

Changing the variable in 2.1 .9 to $\sqrt{V}$, by dividing by $\sqrt{v}$, and assuming $\|g\| \leq c_{2}\|x\|$, ( $c_{2}$ constant) the two equations $2.1 .9 / 2.1 .10$ can be integrated to give the bounds

$$
\sqrt{V}(t) \leq \sqrt{V}(0) \exp (-\alpha t)+\frac{c_{1}}{\alpha} M(P)(1-\exp (-\alpha t))
$$

$$
2.1 .13
$$

and

$$
v(t) \leq v(0) \exp (-\beta t)
$$

where

$$
\alpha=\eta / 2-\sqrt{\mu_{c_{0}}}=\sqrt{\mu}\left(\frac{\Omega}{2 \sqrt{\mu}}-c_{0}\right) \quad 2.1 .15
$$

and

$$
\beta=\eta / 2-\mu_{c_{2}}=\mu\left(\frac{\Omega}{2 \mu}-c_{2}\right) \quad 2.1 .16
$$


Finally, by use of the inequality

$$
\|x\|^{2} m(P) \leq \underline{x}^{T} P \underline{x} \leq M(P)\|\underline{x}\|^{2} \quad 2.1 .17
$$

the two bounds in $V(t)$ above give respectively

$$
\begin{array}{r}
\|\underline{x}(t)\| \leq \sqrt{\mu}\left(\|\underline{x}(0)\| \exp (-\alpha t)+\frac{\left.c_{1}(1-\exp (-\alpha t))\right)}{2.1 .13}\right.
\end{array}
$$

and

$$
\|x(t)\| \leq \sqrt{\mu}\|x(0)\| \exp (-\beta t)
$$

which imply, if $\alpha>0, \beta>0$, that

$$
\|\underline{x}(t)\| \leq \sqrt{\mu} \max \left(\|\underline{x}(0)\|, c_{1} / \alpha\right)
$$

and

$$
\|\underline{x}(t)\| \leq \sqrt{\mu \|} \underline{x}(0) \|
$$

(Note for brevity we have written $\underline{x}(t)=\underline{z}\left(t, \underline{x}_{0}, t_{0}\right)$, $t_{0}=0$ from 1.2.4). The above work follows that of Wiberg (23) with some corrections, namely the bound 2.1.18. A crude RAS from the bounds is given in A3. We add that if $c_{o}$ is given á priori too large, $\alpha$ may be negative which destroys the bound; but if $\underline{G}(x, t) \rightarrow 0$ as $\| \underline{x} \rightarrow 0$ then the bound 2.1 .18 will always hold in some region $R(h)$ for sufficiently small $h$.

Generally, $\alpha^{-1}$ and $\beta^{-1}$ behave as time constants for the respective systems in some region $R(h)$, where $c_{0}$ and $c_{2}$ are considered fixed. As $\mu$ and $\eta$ are complex functions of $Q$, obtaining the analytic maximum of $\alpha$ or $\beta$ is difficult and we resort to the following sub-optimum in each case:
a) $\max \eta$ over $\bar{Q}$ then
b) minimize $\mu$ over a subspace of $\bar{Q}$,

where $\bar{Q}$ is the space of p.d.s. matrices.

Problem (a) was first solved by Lewis and Tausky (24). Problem (b) arises because $Q$ and $P$ giving the 
maximum $\eta$ are not unique, and is the main content of this chapter.

Section 2.2 generalizes some previous work of Wiberg (23). The results of the remaining sections are believed to be new.

\subsection{The Optimum}

$$
\text { Assume A has linear elementary divisors (21), }
$$

i.e there exists a transformation matrix $s$ such that

$$
\mathrm{S}^{-1} \mathrm{AS}=\mathrm{C} \quad 2.2 .1
$$

where $C$ is a diagonal matrix of eigenvalues of $A$ and the columns of $s, \underline{s}_{i}, i=1, n$, are their eigenvectors chosen so that $\left\|\underline{s}_{i}\right\|=1, a 11 i$.

Select $P$ as

$$
\mathrm{P}=\left((\mathrm{SD})(\mathrm{SD})^{*}\right)^{-1}=(\mathrm{SD})^{-*}(\mathrm{SD})^{-1}
$$

$$
2.2 .2
$$

where $*$ denotes conjugate transpose and $D=\operatorname{diag}\left(\mathrm{d}_{1}, \mathrm{~d}_{2}, \ldots\right.$ $\left.\ldots . . ., d_{n}\right)$ is an arbitrary diagonal matrix rith $d_{i} \neq 0$. Then $P$ is a p.d. hermitian matrix and substitution into 2.1 .6 yields

$$
\mathrm{Q}=-(\mathrm{SD})^{-*}\left(\mathrm{C}+\mathrm{C}^{*}\right)(\mathrm{SD})^{-1} \quad 2.2 \cdot 3
$$

which is also p.d. hermitian (p.d.h.) and not necessarily real. Then

$$
\begin{aligned}
\lambda\left(\mathrm{p}^{-1} \mathrm{Q}\right) & =-\lambda\left[(\mathrm{SD})\left(\mathrm{C}+\mathrm{C}^{*}\right)(\mathrm{SD})^{-1}\right] \\
& =-\lambda\left(\mathrm{C}+\mathrm{C}^{*}\right)
\end{aligned}
$$$$
2.2 .4
$$

and

$$
\begin{aligned}
m\left(p^{-1} Q\right)=\eta & =-2 M(A) \\
M\left(p^{-1} Q\right) & =-2 m(A)
\end{aligned}
$$

Vogt (19) has shown that for $P$ and $Q$ satisfying 2.1 .6 we have the inequalities

$$
\begin{array}{ll}
2 M(A) \leq-m\left(P^{-1} Q\right) & 2.2 .6 \\
2 m(A) \geq-M\left(P^{-1} Q\right) & 2.2 .7
\end{array}
$$


the former showing that $h$ in 2.2 .5 is the maximum possible. Statement 2.2 .4 shows with suitable ordering of eigenvalues that $\lambda\left(P^{-1} Q\right)=-2 R e \lambda(A)$.

For a more complete statement consider a result of Barnett (20) who showed that if A is stable, then given a p.d. Q,

$$
A=P^{-1}\left(R-\frac{1}{2} Q\right) \quad 2.2 .8
$$

where $p$ solves 2.1 .6 and $R$ is a skew-symmetric matrix found from 2.2 .8 , but which also solves

$$
A^{T}+R A=\frac{1}{2}\left(A^{T}-Q A\right) \quad 2.2 .9
$$

He gave the bound

$$
\left|\beta_{j}\right| \leq M\left(i^{-1} S\right) \quad(j=\sqrt{-1}) \quad 2.2 .10
$$

where $\lambda_{j}(A)=\alpha_{j}+i \beta_{j}, j=1, \ldots, \pi$.

It easily follows from 2.2 .8 and the $P$ in 2.2.2 that

$$
2 \lambda\left(\mathrm{P}^{-1} \mathrm{R}\right)=\lambda\left(\mathrm{C}-\mathrm{C}^{*}\right)=2 \operatorname{Im} \lambda(\mathrm{A})
$$

showing that equality also holds in 2.2.10. Infact with suitable ordering of eigenvalues

$$
\lambda(\mathrm{A})=\lambda\left(\mathrm{p}^{-1} \mathrm{~s}\right)-\frac{1}{2} \lambda\left(\mathrm{p}^{-1} \mathrm{Q}\right)
$$

Finally, if $A$ is real, $P$ in 2.2 .2 may be taken real. For the $\underline{S}_{i}$ appear, if complex, in conjugate pairs. Suppose $\underline{S}_{i}$ and $\underline{s}_{j}$ are such a pair, then choose $d_{i}=d_{j}$. Then $\exists$ asymetric permutation matrix $T(30)\left(i . e \cdot T^{T}=T^{-1}=T\right.$ ) such that

$$
\text { then } \quad \begin{aligned}
\overline{S D} & =S I T \\
\overline{S D}^{\mathrm{T}} & =(S D)^{*}=T(S D)^{\mathrm{T}} \quad \text { and } \\
\mathrm{P}^{-1} & =(S D)(S D)^{*} \\
& =(S D) T(S D)^{\mathrm{T}}
\end{aligned}
$$

Hence $\mathrm{P}^{-1}$, and thus $\mathrm{P}$, are symmetric and thus real. 


\subsection{The Minimum Condition Number, (P)}

The matrix $P$ in 2.2 .2 contains essentially $n-1$

arbitrary parameters to a multiplicative constant. Regarding $\mu$ as a function of $P$ and thus $D$, problem (b) is restated as

$$
\min _{\mathrm{D}} \mu(\mathrm{P}) \quad 2.3 .1
$$

This quantity $\mu$ is called the $\mathrm{p}$-condition number of $\mathrm{p}$ and is related to the condition number $K(x)$ of a general matrix $\mathrm{X}$ defined as (Bauer (22).)

$$
K(\mathrm{X})=\|\mathrm{x}\|\|\mathrm{x}-1\|
$$

$$
\text { where } \begin{array}{rlr}
\|x\| & =\sup _{x \neq 0}\|x\| /\|x\| & 2.3 .7 \\
& =\left(M\left(X^{*} x\right)\right)^{\frac{1}{2}} & 2.3 .3
\end{array}
$$

For Hermitian $x, \mu(x)=K(x)$ and for $P$ in 2.2 .2

$$
\begin{aligned}
K(P)=K(P-1) & =K\left((S D)(S D)^{*}\right) \\
& =K\left((S D)^{*}(S D)\right) \\
& =K^{2}(S D)
\end{aligned}
$$

Thus $\min _{D} \mu(P)=\min _{D} K^{2}(S D)$ $2 \cdot 3 \cdot 10$

Now define $|X|$ as the matrix whose $i, j$ th element is $\left|x_{i, j}\right|$. Such a matrix is called non-negative. We say $X$ has checkerboard sign distributions (CSD) if matrices $\mathbb{F}_{1}$ and $E_{2}$ exist such that $S=E_{1}|S| E_{2}$ with $\left|E_{1}\right|=\left|E_{2}\right|=I$. We shal1 need the following theorem due to Bauer (22).

Theorem 2.3

For the matrix. norm $\|\cdot\|$ in 2.3 .8

$$
\min _{D} K(S D) \leq\left\||S| \mid s^{-\pi}\right\|
$$

with equality holding if both $S$ and $S^{-1}$ have CSD.

This theoren gives a useful bound on the minimum in $2 \cdot 3 \cdot 10$ 
2.4 A Conjecture of Viberg (23)

Wiberg conjectured that $\mu(P)$ is minimized if $D=I$ (i.e $\left\|s_{j}\right\|=1$ ). Although this is true for $n=2$ it is false for $n>2$.

Choose $A=S^{-1} \mathrm{CS}$ where

$$
s=\left[\begin{array}{ccc}
1, & 1 / \sqrt{2}, & 1 / \sqrt{6} \\
0, & 1 / \sqrt{2}, 2 / \sqrt{6} \\
0, & 0,1 / \sqrt{6}
\end{array}\right] \quad \mathrm{C}=\operatorname{diag}(-1,-2,-3)
$$

With $D=I, \mu(P)=K^{2}(S D)=32.52$

Now

$$
S^{-1}=\left[\begin{array}{rrr}
1 & -1 & 1 \\
0 & \sqrt{2} & -2 \sqrt{2} \\
0 & 0 & \sqrt{6}
\end{array}\right]
$$

Since both $S$ and $S^{-1}$ have CSD, by Theorem 2.3

$$
\begin{aligned}
\min _{D} K(S D) & =\left\||S|\left|S^{-1}\right|\right\| \\
& =(3+\sqrt{10})
\end{aligned}
$$

Then $\mu(P)=K^{2}(S D)=(3+\sqrt{10})^{2}=37.274$

which disproves the conjecture.

\subsection{An Optinum Class of Matrices}

The diagonal matrix $D$ giving the upper bound in 2.3.11 is given in $A 3$ and would appear a better choice than $D=I$. A natural question is what form must $A$ have in 2.1 .1 and 2.1 .2 so that $S$ has $\mathrm{CSD}$. We have:

\section{Theoren 2.5}

If $A$ is a stable matrix in compaion . form with distinct real eigenvalues then $S$ and $S^{-1}$ may bo chosen to have CSD. 


\section{Proof}

For Ain companion form (CF)

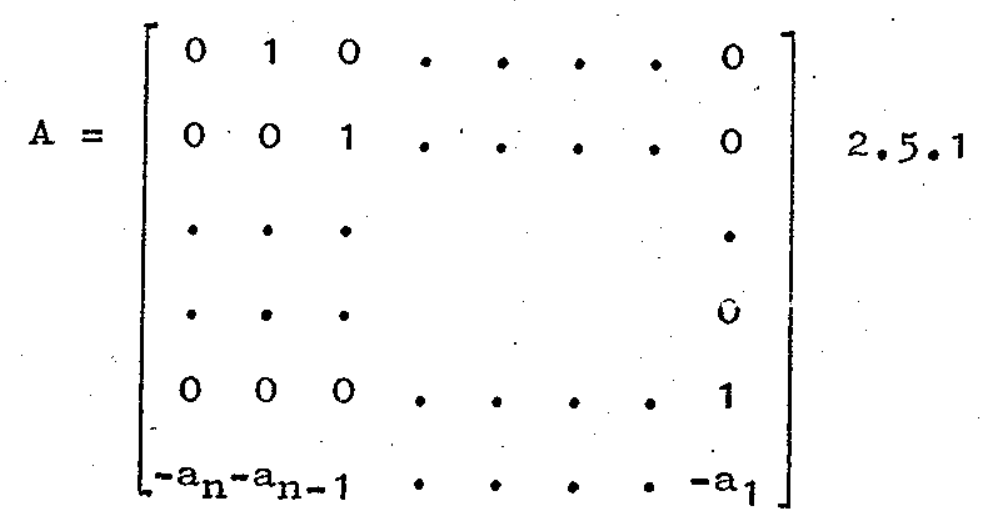

with $\lambda_{i}(A)=-\alpha_{i}<0, i=1, \ldots n$, we choose $s=v_{n}$ (and disregard unit columns for convenience) where $V_{n}$ is the Van der Monde matrix (30)

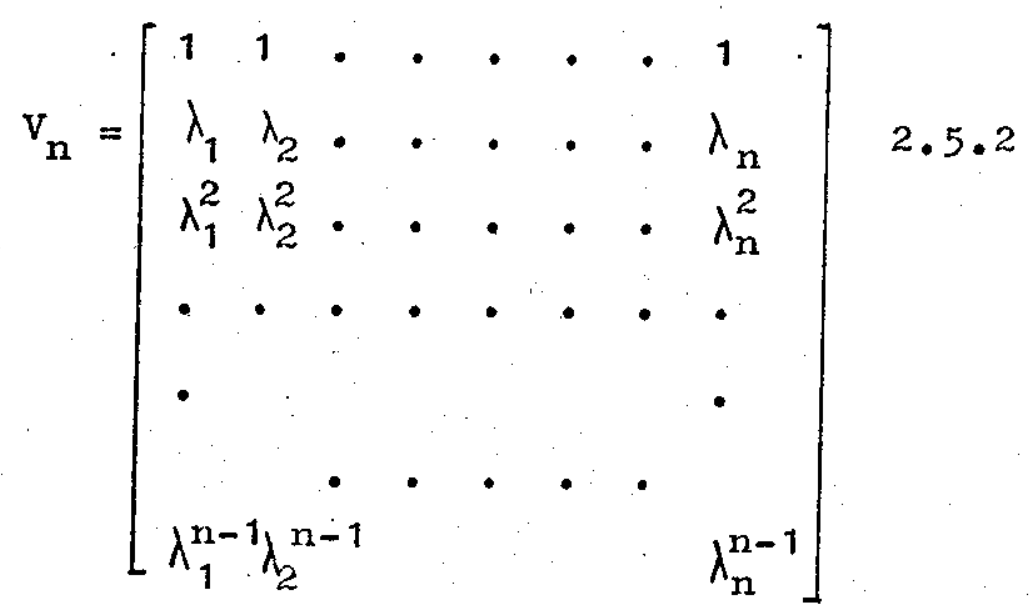

$v_{n}$ is clearly CSD.i.e.

$$
\mathrm{E}_{1} \mathrm{v}_{\mathrm{n}}=\left|\mathrm{v}_{\mathrm{n}}\right|
$$

with $E_{1}=\operatorname{diag}\left(1,-1,1, \ldots,(-1)^{n-1}\right)$

To show that $\mathrm{v}_{\mathrm{n}}^{-1}$ isss let $\sigma_{\mathrm{m}}$, where

$$
\begin{aligned}
\sigma_{\mathrm{m}} & =\sigma_{\mathrm{m}}\left(\mathrm{y}_{1}, \mathrm{y}_{2}, \ldots, \mathrm{y}_{\mathrm{n}}\right), \quad 1 \leq \mathrm{m} \leq \mathrm{n} \\
& =\sum \mathrm{y}_{\mathrm{v}_{1}} \mathrm{y}_{\mathrm{v}_{2}} \cdots \mathrm{y}_{\mathrm{n}} \\
\sigma_{\mathrm{o}} & =1
\end{aligned}
$$

be the $m^{\text {th }}$ elementary symmetric function of any $n$ variables $\mathrm{y}_{i}(30), e \cdot g \cdot \sigma_{1}=\sum^{n} \mathrm{y}_{i}$ and $\sigma_{n}=\prod^{n} \mathrm{y}_{i} \cdot$ Also define

$$
\sigma_{\mathrm{m}}^{\mathrm{p}}=\sigma_{\mathrm{m}}^{\mathrm{p}}\left(\mathrm{y}_{1}, \mathrm{y}_{2}, \ldots \mathrm{y}_{\mathrm{p}-1}, \mathrm{y}_{\mathrm{p}+1}, \ldots \mathrm{y}_{\mathrm{n}}\right)
$$

as the $m^{\text {th }}$ elementary symmetric function of the $y_{i}$ with $y_{p}$ missing. Then the $i, j^{\text {th }}$ element of $v_{n}^{-1}, v_{i j}^{-1}$, is given 
by $(28)$

$$
v_{i j}^{-1}=(-1)^{j-1} \frac{\sigma_{11-j}^{i}\left(\lambda_{1}, \lambda_{2}, \ldots, \lambda_{n}\right)}{\prod_{k \neq 1}^{n}\left(\lambda_{K}-\lambda_{i}\right)} \quad 2.5 .5
$$

Numerically stable formulae exist to invert $V_{n}$ (39)

Assume that $0_{i=1} \alpha_{1}<\alpha_{2} \quad \ddot{m}<\alpha_{n}$ and let

$$
\left.r_{i}=\prod_{j=1}^{i=1}\left(\alpha_{i}-\alpha_{j}\right)\right]_{j=i+1}^{n_{j}}\left(\alpha_{j}-\alpha_{i}\right)>0 \quad 2.5 .6
$$

Then in terms of $\alpha_{i}, v_{n}^{-1}$ may be written

$$
\mathrm{V}_{\mathrm{n}}^{-1}=\mathrm{RQ}
$$

with $R=\operatorname{diag}\left(\frac{1}{x_{1}},-\frac{1}{r_{2}}, \cdots, \frac{(-1)^{n-1}}{r_{n}}\right)$

and

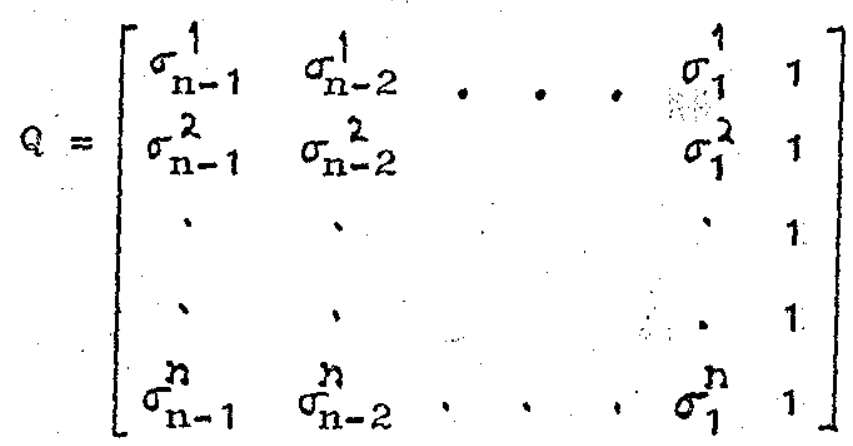

with $q_{i, j}=\sigma_{n-j}^{i}\left(\alpha_{1}, \ldots, \alpha_{n}\right)>0$. Clearly, all elements of $Q$ are non-negative and

$$
\left.\mathrm{v}_{\mathrm{n}}^{-1}=\mathrm{E}_{1} \mid \mathrm{v}_{\mathrm{n}}^{-1}\right\}=\mathrm{E}_{1} \mid \mathrm{RQ}
$$

with $E_{1}$ from 2.5.4. Hence the theorem is proved.

In general the CSD property does not hold. It is not satisfied, for exaple, when $A$ is in $C F$ with complex $\lambda(A)$. In such cases one resorts to a non-linear programming technique to minimize $\mu$ (section 2.8 ) or chooses some upper bound such as that of Bauer, 2.3.11. In this respect some simple bounds can be found. 


\subsection{Bounds on $\mu(1)$}

The best bound found by the author was from a result of Marcus and Haynsworth(27). They showed

$$
K(p)=\mu(p) \leq \frac{1+\sqrt{1-D_{1}}}{1-\sqrt{1-D_{1}}}
$$

where

$$
D_{1}=a(p) /\left(\frac{t(p)}{n}\right)^{n}
$$

and $d(P), t(P)$ are the determinant and trace of $P$ respectively. Let

$$
F(P)=\frac{1+\sqrt{1-D_{1}}}{1-\sqrt{1-D_{1}}}
$$

then in general $F(P) \neq F\left(P^{-1}\right)$ and two bounds are possible in 2.6.1. Using the arithmetic-geometric mean inequality (2.6.12), each bound is minimized, for the choice of $p$ in 2.2 .2 , when $D=I$ and $d_{i}=\left\|r_{i}\right\|$ respectively and we have

$$
\mu\left(\mathrm{P}^{-1}\right) \leq F\left(\mathrm{P}^{-1}\right)
$$$$
2.6 .4
$$

and

$$
\mu(P) \leq F(P)
$$

where

$$
\begin{aligned}
D_{1}\left(p^{-1}\right) & =|d(s)|^{2} / \prod_{i=1}^{n}\left\|s_{i}\right\|^{2} \\
& =|d(s)|^{2}
\end{aligned}
$$

and

$$
D_{1}(p)=\left(|d(s)|^{2} \prod_{i=1}^{n}\left\|\underline{r}_{i}\right\|^{2}\right)^{-1}
$$

Here $\underline{r}_{i}=$ row $i$ or $\mathrm{s}^{-1}$.

The only difficult calculation in 2.6 .6 or 2.6 .7 is $d(s)$. Simplification arises with $A$ in $C F$ for then

$$
\begin{aligned}
& a\left(v_{n}\right)=\prod_{j<j}\left(\lambda_{j}-\lambda_{i}\right) \text { and } \\
& D_{1}\left(p^{-1}\right)=\prod_{i<j}\left(\left|\lambda_{j}-\lambda_{i}\right| 2\right) \prod_{j=1}^{n}\left(\sum_{j=1}^{n} \mid \lambda_{i} 2(j-1)\right) 2.6 .8
\end{aligned}
$$




$$
D_{1}(P)=\prod_{j<j}\left|\lambda_{j}-\lambda_{i}\right|^{2} / \prod_{j=1}^{n}\left(\sum_{j=1}^{n}\left|\sigma_{n-j}^{j}\right|^{2}\right) \quad 2.6 .9
$$

(The latter follows because in 2.5.7 $|\mathrm{d}(\mathrm{R})|=\left|\mathrm{a}\left(\mathrm{v}_{\mathrm{n}}\right)\right|^{-2}$ )

Note that equality occurs in 2.6 .1 for $n=2$.

A looser bound than 2.6 .1 is proved as follows.

Consider $P$ in 2.2 .2 with unit vectors $\underline{s}_{i},\left\|\underline{s}_{i}\right\|=1$, and $\mathrm{D}=\mathrm{I}$. Then let $\mathrm{u}_{i}, i=1, \ldots, \mathrm{n}$, be the eigenvectors of $\mathrm{T}\left(\mathrm{T}=\mathrm{S}^{*} \mathrm{~g}\right)$ ordered as $\mathrm{u}_{1} \geq \mathrm{u}_{2} \geq \ldots \geq \mathrm{u}_{\mathrm{n}}$. Since $\mu(p)=K(T)$,

$$
K(T)=\frac{u_{1}}{u_{n}}
$$

If $u$ is any eigenvalue of $T$, by Gershgorin's Theorem (30)

$$
|u-| s_{i}{ }^{*} \underline{s}_{j}||=|u-1| \leq \rho_{i}, i=1, n
$$

where

Then

$$
\rho_{i}=\sum_{j \neq j}^{n}\left|t_{i, j}\right|=\sum_{j \neq 1}^{n}\left|s_{i}^{*} \underline{s}_{j}\right|
$$

$$
u_{1} \leq 1+\max _{i} \rho_{i}=1+\bar{\rho}
$$

Now

$$
\begin{aligned}
\dot{d}(T) & =\prod_{j=1}^{n} u_{i=1}^{i} \\
& =u_{n} \prod_{j=1} u_{i}
\end{aligned}
$$

Using the Arithmetic-Geometric mean inequality

$$
\left(T^{n} T_{i}\right)^{\frac{1}{n}}=G_{n} \leq A_{n}=\left(\sum_{i}^{n} u_{i}\right) / n \quad 2.6 .12
$$

we have, since $\sum u_{i}=n$,

$$
d(T) \leq u_{n} \frac{\left(n-u_{n}\right)^{n-1}}{n-1}
$$

$$
\leq u_{n}\left(\frac{n}{n-1}\right)^{n-1}
$$

Substitution of 2.6 .11 and 2.6 .13 into 2.6 .10 yields the bound

$$
\mu(P) \leq \frac{1}{T d(s) T^{2}}\left[\frac{n}{n-1}\right]^{n-1}(1+\bar{\rho}) \quad 2.6 .14
$$


used Gersherorin's inequality to obtain

$$
\mu(P) \leq \frac{1+\bar{\rho}}{1-\bar{\rho}}
$$

To show the bound is sometimes invalid consider $A$ and $S$ where

$$
A=-\left[\begin{array}{ccc}
1 & 12 / 5 & 24 / 6 \\
0 & 2 & 0 \\
0 & 0 & 3
\end{array}\right] \quad S=\left[\begin{array}{lll}
1 & 12 / 13 & 12 / 13 \\
0 & 5 / 13 & 0 \\
0 & 0 & 5 / 13
\end{array}\right]
$$

for which $S^{-1} A S=\operatorname{diag}(-1,-2,-3) \cdot \operatorname{clear1y}, \bar{\rho}=24 / 13>1$ and the bound becomes negative.

\subsection{Non-uniqueness of $P$ in the Form 2.2.2}

The form of $\mathrm{p}$ in 2.2 .2 which satisfies the condition

$$
\eta=m\left(P^{-1} Q\right)=-2 M(A) \quad 2.7 .1
$$

is not generally unique. We only need consider A stable and in the form

$$
A=\left[\begin{array}{ccc}
-a & 1 & 0 \\
-1 & - \\
1 & \\
0 & 1 & A_{1}
\end{array}\right] \quad 2.7 .2
$$

where $A_{1}$ is $(n-1) X(n-1)$ such that $S_{1}^{-1} A_{1} S_{1}^{-1}=C_{1}$. Let $M(A)=-a, M\left(A_{1}\right)=-a_{1}$ and $m\left(A_{1}\right)=-a_{2} \quad\left(a<a_{1}\right)$. $P$ and $Q$ are of the form

$$
P=\left[\begin{array}{ccc}
1 & 1 & 0 \\
--1 & - & - \\
0 & 1 & P_{1}
\end{array}\right] \quad Q=\left[\begin{array}{ccc}
2 a & 1 & 0 \\
-1 & 1 & - \\
0 & 1 & Q_{1}
\end{array}\right]
$$

where $A_{1}^{T} P_{1}+P_{1} A_{1}=-Q_{1}$. Then condition 2.7 .1 is met with. $\mathrm{P}_{1}^{-1}=\left(S_{1} D_{1}\right)\left(S_{1} D_{1}\right)^{*}$ for which $h\left(Q_{1}\right)=m\left(P_{1}^{-1} Q_{1}\right)=2 a_{1}$ and 
$M\left(P_{1}^{-1} Q_{1}\right)=2 a_{2}$. By varying $P_{1}$ and $Q_{1}$ and noting that $\eta$ depends continuously on the elements of $P_{1}$, a $P_{1}$ not generally of the form 2.2 .2 can be found such that

$$
2 \mathrm{a}<\mathrm{m}\left(\mathrm{P}_{1}^{-1} \mathrm{Q}_{1}\right)<2 \mathrm{a}_{1}
$$

Then we still have $\mathrm{m}\left(\mathrm{P}^{-1} \mathrm{Q}\right)=2 \mathrm{a}$, if $\mathrm{a}<\mathrm{a}_{1}$, but $\mathrm{p}$ not of the form 2.2.2.

\section{Example}

Choose

$$
A=\left[\begin{array}{cccc}
-a & 1 & 0 & 0 \\
- & - & - & - \\
0 & 1 & 0 & 1 \\
0 & 1 & -1 & -1
\end{array}\right] \quad \lambda=-a,-\frac{1}{2}(1 \mp \sqrt{3} i)
$$

Hexe, the only real minimizing $p_{1}$, to a constant factor, is

$$
P_{1}=\sqrt{\frac{1}{5}}\left[\begin{array}{ll}
1 & \frac{1}{2} \\
\frac{1}{2} & 1
\end{array}\right], \quad \mu\left(p_{1}\right)=3
$$

but with the choice

$$
p_{1}=\frac{1}{2}\left[\begin{array}{ll}
3 & 1 \\
1 & 2
\end{array}\right]
$$

$M\left(P_{1}^{-1} Q_{1}\right)=1+1 / \sqrt{ } 5$ and $m\left(P_{1}^{-1} Q_{1}\right)=1-1 / \sqrt{ } 5$ and 2.7 .4 is satisfied if $2 a<1-1 / \sqrt{ } 5$.

A1so

$$
\mu(\mathrm{P})=\frac{\sqrt{5}+1}{\sqrt{5}-1}<3
$$

and this $P_{1}$ gives smaller values of $\alpha$ or $\beta$ in $2.1 .15 / 16$. Thus problem (b) does not necessarily give 'best' suboptima of $\alpha$ or $\beta$.

The arbitrariness of the form 2.2 .2 is due to the somewhat non-unique transformation $S$ in 2.2 .1 . It can generate a subspace of p.d matrices ( $P$ ) giving the same 
value of $\eta$. To see this, consider a solution $P$ of 2.1 .6 , for a given p.d.s $Q$, in terms of the $\underline{s}_{j}$ and $\lambda_{i}$ of $A(31)$

$$
\mathrm{p}=\left(\mathrm{S}^{*}\right)^{-1} \mathrm{P}_{1} \mathrm{~S}^{-1} \quad 2.7 .5
$$

where

$$
\left(p_{1}\right)_{j}=-s_{i}^{*}{ }^{*} \underline{s}_{j} /\left(\bar{\lambda}_{i}+\lambda_{j}\right) \quad 2.7 .6
$$

Let $\eta_{p}=m\left(p^{-1} Q\right)$, then $p_{0}$ arid $Q_{0}$ also solve 2.1.6, with $D$ arbitrary and diagonal, where $P_{0}=(S D)^{-*} P_{1}(S D)^{-1}$ and $Q_{0}=(S D)^{*}\left(S^{*} Q S\right)(S D)^{-1}$. Further

$$
\mathrm{P}_{0}^{-1} \mathrm{Q}_{\mathrm{O}}=(\mathrm{SD}) \mathrm{P}_{1}^{-1} \mathrm{~S}^{*} \mathrm{QS}(\mathrm{SD})^{-1}
$$

and due to similarity properties

$$
\begin{aligned}
\lambda\left(P_{0}^{-1} Q_{0}\right) & =\lambda\left(P_{1}^{-1} S^{*} Q S\right)=\lambda\left(S P_{1}^{-1} s^{*} Q\right) \\
& =\lambda\left(P^{-1} Q\right)
\end{aligned}
$$

Then $n_{p}=n_{p_{o}}$

\subsection{Numerical Optimum of the Condition Number}

In view of the fact that for $n=2$, the $P$ of the form 2.2 .2 which minimizes $\mu$ is real, it is reasonable to conjecture that this holds generally. To test the conjecture, Powell's conjugate gradient algorithm (A4) was used to minimize $\mu(p)$ over the $N$-dimensional space of elements of $D$, where for

(a) real $\mathrm{P}, \mathrm{N}=\mathrm{n}-\mathrm{k}-1$;

(b) hermitian $P, N=\mathrm{n}-1$;

where $\lambda_{j}=r_{j} \mp_{i s_{j}}, j=1,2, \ldots, k$

$\lambda_{j}=-r_{j}<0, j=2 k+1, \ldots, n$

are the eigenvalues of $\mathrm{A}$. The bounds $2.3 .11,2.6 .4$ and 2.6 .5 were also calculated.

Table 2.1 shows some results for a number of third and fourth order matrices (A1 to A10). The average number. of function evaluations of $\mu(p)$ for an accuracy of $10^{-3}$ 
TABLE 2.1

\begin{tabular}{|c|c|c|c|c|c|c|c|c|c|}
\hline $\begin{array}{l}\text { MATRIX A } \\
\text { (LAST ROW ONLY } \\
\text { GIVIEN IF A IS IN } \\
\text { C.I.) }\end{array}$ & 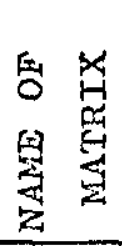 & $F(P)$ & $F\left(P^{-1}\right)$ & $\||s| \mid s-1\|$ & $\left\{\begin{array}{l}p=\left(s s^{4}\right)^{-1} \\
\left\|s_{i}\right\|=1 \\
\text { (WIBERG) }\end{array}\right.$ & $\begin{array}{l}\text { MIN } \mu(P) \\
P \text { REAL }\end{array}$ & $\mathbb{N}$ & MIN $\mu(P)$ & $\mathbf{H}$ \\
\hline $\begin{array}{rrr}-8 & 1 & 5 \\
4 & -4 & 2 \\
-18 & 5 & 7\end{array}$ & \multirow[t]{2}{*}{ A1 } & \multirow[t]{2}{*}{$\begin{array}{r}.1208 \\
\times \quad 10^{4}\end{array}$} & \multirow[t]{2}{*}{$\begin{array}{l}14799 \\
\times 10^{3}\end{array}$} & \multirow[t]{2}{*}{$\begin{array}{l}.19261 \\
\times 10^{3}\end{array}$} & \multirow[t]{2}{*}{$\begin{array}{l}.14435 \\
\times 10^{3}\end{array}$} & \multirow[t]{2}{*}{$\begin{array}{l}.14345 \\
\times 10^{3}\end{array}$} & \multirow[t]{2}{*}{1} & \multirow[t]{2}{*}{$\begin{array}{l}.14345 \\
\times 10^{3}\end{array}$} & \multirow[t]{2}{*}{2} \\
\hline$\lambda=-2 \mp 4 i,-1$ & & & & & & & & & \\
\hline $\begin{array}{rrr}-33 & -16 & -72 \\
24 & 10 & 57 \\
8 & 4 & 17\end{array}$ & \multirow[t]{2}{*}{ A2 } & \multirow[t]{2}{*}{$\begin{array}{l}.58282 \\
\times 10^{5}\end{array}$} & \multirow[t]{2}{*}{$\begin{array}{l}.17661 \\
\times 10^{8}\end{array}$} & \multirow[t]{2}{*}{$\begin{array}{r}.2789 \\
\times 10^{5}\end{array}$} & \multirow[t]{2}{*}{$\begin{array}{r}.3238 \\
\times \quad 10^{5}\end{array}$} & \multirow[t]{2}{*}{$\begin{array}{l}26178 \\
\times 10^{5}\end{array}$} & \multirow[t]{2}{*}{2} & \multirow[t]{2}{*}{ SAME } & \multirow[t]{2}{*}{2} \\
\hline$\lambda=-1,-2,-3$ & & & & & & & & & \\
\hline $\begin{array}{rrr}-4 & -1 & -1 \\
-2 & -4 & -1 \\
0 & -1 & -4 \\
\end{array}$ & \multirow[t]{2}{*}{ A3 } & \multirow[t]{2}{*}{$\begin{array}{l}.29242 \\
\times 10^{4}\end{array}$} & \multirow[t]{2}{*}{$\begin{array}{l}.21561 \\
\times 10^{3}\end{array}$} & \multirow[t]{2}{*}{$\begin{array}{l}.22456 \\
\times 10^{3}\end{array}$} & \multirow[t]{2}{*}{$\begin{array}{l}.21304 \\
x \cdot 10^{3}\end{array}$} & \multirow[t]{2}{*}{$\begin{array}{l}.200781 \\
\times 10^{3}\end{array}$} & \multirow[t]{2}{*}{2} & \multirow[t]{2}{*}{ SAME } & \multirow[t]{2}{*}{2} \\
\hline$\lambda=-3,-3,-6$ & & & & & & & & & \\
\hline$-101,-103,-3$ & \multirow[t]{2}{*}{ A4 } & \multirow[t]{2}{*}{$\begin{array}{l}.242 \\
\times \quad 10^{4}\end{array}$} & \multirow[t]{2}{*}{$\begin{array}{r}.3165 \\
\times \quad 10^{3}\end{array}$} & \multirow[t]{2}{*}{$\begin{array}{l}.23774 \\
\times 10^{3}\end{array}$} & \multirow[t]{2}{*}{$\begin{array}{l}.26098 \\
\times 10^{3}\end{array}$} & \multirow[t]{2}{*}{$\begin{array}{l}.231718 \\
\times 10^{3}\end{array}$} & \multirow[t]{2}{*}{1} & \multirow[t]{2}{*}{$\begin{array}{l}.231756 \\
\times 10^{3}\end{array}$} & 2 \\
\hline$\lambda=-1,-1 \mp 10 i$ & & & & & & & & & \\
\hline$-200,-202,-102$ & A5 & 93.085 & 48.984 & 50.886 & 42.269 & 41.7843 & 1 & 41.7854 & 2 \\
\hline$\lambda=-100,-1 \mp i$ & & & & & & & & & \\
\hline
\end{tabular}

* Derogatory 
TABLE 2.1 (contd.)

\begin{tabular}{|c|c|c|c|c|c|c|c|c|c|}
\hline$-4,-10,-10,-5$ & A6 & $\begin{array}{r}.54903 \\
\times 10^{7}\end{array}$ & $\begin{array}{l}19123 \\
\times 10^{5}\end{array}$ & $\begin{array}{r}.37229 \\
\times 10^{4}\end{array}$ & $\begin{array}{r}.34319 \\
\times 10^{4}\end{array}$ & $\begin{array}{l}.315129 \\
\times 10^{4}\end{array}$ & 2 & $\begin{array}{l}.315984 \\
\times 10^{4}\end{array}$ & 3 \\
\hline \multicolumn{10}{|l|}{$\lambda=-2,-1,-1 \mp i$} \\
\hline$-10,-18,-15,-8$ & \multirow{2}{*}{$\begin{array}{r}\text { A7 } \\
84 i \\
\end{array}$} & \multirow[t]{2}{*}{$\begin{array}{l}.485 \\
\times \quad 10^{3}\end{array}$} & \multirow[t]{2}{*}{$\begin{array}{r}2255 \\
\times \quad 10^{3}\end{array}$} & \multirow[t]{2}{*}{$\begin{array}{l}.20411 \\
\times 10^{3}\end{array}$} & \multirow[t]{2}{*}{$\begin{array}{l}15029 \\
\times 10^{3}\end{array}$} & \multirow[t]{2}{*}{$\begin{array}{l}.14704 \\
\times 10^{3}\end{array}$} & \multirow[t]{2}{*}{2} & \multirow[t]{2}{*}{$\begin{array}{r}.14704 \\
\times 10^{3}\end{array}$} & \multirow[t]{2}{*}{3} \\
\hline$\lambda=-5.94,-1,-.532 \mp 1$ & & & & & & & & & \\
\hline$-202,-402,-304,-103$ & \multirow[t]{2}{*}{ A. 8} & \multirow[t]{2}{*}{$\begin{array}{l}19598 \\
\times 10^{5}\end{array}$} & \multirow[t]{2}{*}{$\begin{array}{l}.96673 \\
\times 10^{3}\end{array}$} & \multirow[t]{2}{*}{$\begin{array}{l}.44055 \\
\times 10^{3}\end{array}$} & \multirow[t]{2}{*}{$\begin{array}{r}.3453 \\
\times 10^{3}\end{array}$} & \multirow[t]{2}{*}{$\begin{array}{l}34353 \\
\times 10^{3}\end{array}$} & \multirow[t]{2}{*}{2} & \multirow{2}{*}{$\begin{array}{r}34354 \\
\times \quad 10^{3}\end{array}$} & \multirow[t]{2}{*}{3} \\
\hline$\lambda=-100,-1,-1 \mp i$ & & & & & & & & & \\
\hline$-24,-50,-35,-10$ & \multirow[t]{2}{*}{ A9 } & \multirow[t]{2}{*}{$\begin{array}{r}.13794 \\
\times 10^{12}\end{array}$} & \multirow[t]{2}{*}{$\begin{array}{r}.33835 \\
\times 10^{8}\end{array}$} & \multirow[t]{2}{*}{$\begin{array}{l}.27130 \\
\times 10^{6}\end{array}$} & \multirow[t]{2}{*}{$\begin{array}{l}.35344 \\
\times 10^{6}\end{array}$} & \multirow[t]{2}{*}{$\begin{array}{r}27130 \\
\times 10^{6}\end{array}$} & \multirow[t]{2}{*}{3} & \multirow[t]{2}{*}{$\begin{array}{l}27130 \\
\times 10^{6}\end{array}$} & \multirow[t]{2}{*}{3} \\
\hline$\lambda=-1,-2,-3,-4$ & & & & & & & & & \\
\hline$-202,-206,-107,-4$ & \multirow[t]{2}{*}{ A 10} & \multirow[t]{2}{*}{$\begin{array}{r}.7066 \\
\times 10^{6}\end{array}$} & \multirow[t]{2}{*}{$\begin{array}{c}.63339 \\
\times 10^{4}\end{array}$} & \multirow[t]{2}{*}{$\begin{array}{r}13486 \\
\times 10^{4}\end{array}$} & \multirow[t]{2}{*}{$\begin{array}{c}16222 \\
\times 10^{4}\end{array}$} & \multirow[t]{2}{*}{$\begin{array}{r}12751 \\
\times 10^{4}\end{array}$} & \multirow[t]{2}{*}{1} & \multirow[t]{2}{*}{$\begin{array}{c}.12747 \\
\times 10^{4}\end{array}$} & \multirow[t]{2}{*}{3} \\
\hline$\lambda=-1 \mp 10 i,-1 \mp i$ & & & & & & & & & \\
\hline
\end{tabular}


in the minimum was 54 and 76 for $N=2$ and $N=3$ respectively.

Comparison of the minimum $\mu$ for real and complex $P$ indicates that the conjecture may be true; certainly no advantage is gained by minimizing over the higher dimensional space of hermitian $P$ as

of the two bounds $F(P)$ and $F\left(P^{-1}\right)$, the lattex gave better results and, except for matrices $A_{1}, A_{3}$, and $A_{5}$, both were inferior to Bauer's bound. Choosing $\mu$ with $D=I$ (Wibetg) gave some better bounds than that of Bauer (6 against 4 ), but as shown in theory, the latter gave the exact minimum for matrix $A_{9}$.

The examples were chosen with differing eigenvalue spreads and, for most, the minima for $\mu$ seem quite large. One might therefore expect quite crude estimates of the system response, independant of the non-linear terms in 2.1 .1 and 2.1 .2 .

\subsection{C in Jordan Form}

When the elementary divisors of $A$ are non-linear the analysis in 2.2 remains the same except that $C$ is now in Jordan form and the relation 2.2 .5 is replaced by $(\operatorname{vogt}(19))$

$$
-2 M(A)-\epsilon \leq \eta \leq-2 M(A)+\epsilon
$$

with $\epsilon>0$ as small as desired. In theory one has to find the Jordan transformation S. In practice it is simpler to perturb the elements of A slightly, and one can replace $A$ by $\bar{A}$ where $\vec{A}=A-D$ and $D$ a diagonal matrix with sufficiently small positive elements, $d_{i}$, such that $M(\bar{A})<0$ and the eigenvalues $\lambda(\bar{A})$ are distinct. From 
continuity arguments, the optima of $\eta$ and $\mu$ for $\bar{A}$ will differ only slightly from those of A. Sometimes, nonlinear divisors cause little bother since round off errors in computation render the divisors linear (see example A3 Table 2.1)

\subsection{Some Numerical Experiments}

Prior to the work of this chapter the optimization techniques of Rosenbrock and Powell were applied to determine the minima of the quantities: (a) $s\left(P^{-1} Q\right)$, (b) $\mu(p),(c)-\eta$ and (d) $-\eta^{2}$ hs; the latter being important quantities in 2.1 .15 and 2.1 .16 . The quantity $s\left(P^{-1} Q\right)$ is the spread of $\mathrm{P}^{-1} \mathrm{Q}$,

$$
s\left(P^{-1} Q\right)=M\left(P^{-1} Q\right)-m\left(P^{-1} Q\right)
$$

which is a bound on $M(A)-m(A)$, the spread of Re $\lambda_{(A)}$. An algorithm for minimizing (a) to (d) over p.d.s $Q$ is given in A3. The main motivation was to test the usefulness of the bounds 2.2 .6 and 2.2 .7 in locating Re $\lambda(A)$.

Table 2.2 shows some results applied to third order matrices, $u_{i}, i=1,3$ being the eigenvalues of $\frac{1}{2} p^{-1} Q$ $\left(u_{1}=u_{2}=u_{3}\right)$. Here $s\left(P^{-1} Q\right)$ is minimized and average computation time was $110 \mathrm{mill} / \mathrm{sec}$ (I.C.L 1905) for 300 function evaluations ( $F E$ ). In each case $Q=I$ initially. The bounds for matrices $\mathrm{A}_{3}, \mathrm{~B}_{3}$ and $\mathrm{B}_{4}$ are good whereas for $A_{1}, A_{2}$ and $B_{5}$ there was a tendency for two $u_{i}$ to becone equal thus destroying the bound. This was particularly so for conjugate $\lambda(A)$ and sugrests some theoretical reason for occuring. In ali cases the minimizing Q was non-unique. The results of minimizing quantities (b) to (d) are shown graphically in Fig 2.1 to 2.3 by plotting $h / 2$ vs $\mu$ at stages in the mininization. Three matrices ( $A_{1}$ to $A_{3}$ ) 
TABIE 2.2

Minimization of $s\left(\mathrm{P}^{-1} \mathrm{Q}\right)$

\begin{tabular}{|c|c|c|c|c|}
\hline $\begin{array}{l}\text { A } \\
\text { (LAST ROW GIVEN IF } \\
\text { A IN CF }(2.5 .1))\end{array}$ & $\begin{array}{l}\text { NAME OF } \\
\text { MATRIX }\end{array}$ & $\lambda(\mathrm{A})$ & $\begin{array}{r}\frac{1}{2} \lambda\left(P^{-1} Q\right) \\
u_{1}, u_{2}, u_{3}\end{array}$ & $\begin{array}{c}\frac{1}{2} s\left(P^{-1} Q\right) \\
300 \text { FUNCTEON } \\
\text { EVALUATTONS }\end{array}$ \\
\hline $10^{-6}, 0.01002,-.2001$ & B1. & $-10^{-4},-.1,-.1$ & $1002, .0998,6 \times 10^{-6}$ & .09988 \\
\hline $10^{4}, 1110.0,-111.0$ & $\mathrm{~B} 2$ & $-100,0,-10,0,-1$ & $100.35,10.64, .006$ & $100 \cdot 35$ \\
\hline A3 TABLE 2.1 & A3 & $-6,-3,-3$ & $5.004,3.0042,2.9959$ & 3.0034 \\
\hline$\cdot \cdot$ & A1 & $-2 \mp 4 i,-1$ & $2.9906,2.4906, .019$ & 2.4716 \\
\hline A2 & $\mathrm{A} 2$ & $-1,-2,-3$ & $3.003,2.997,10^{-4}$ & 3.002 \\
\hline-10 & & & & \\
\hline $\begin{array}{rrr}3 & -3 & 0 \\
2 & 1 & -1 \\
\end{array}$ & B3 & $-10,-3,-1$ & $9.9899,3.024, .9957$ & 8.0942 \\
\hline $\begin{array}{ccc}-1 & 0 & -.01 \\
-0.1 & -1 & 0 \\
0 & -1 & -1 \\
\end{array}$ & B4. & $-1.1,-.95 \div \frac{.053 i}{2}$ & $1.102, .949, .949$ & .09153 \\
\hline$-200,220,-21$ & B5 & $-10 \mp 101,-1$ & $10.46,10.46, .089$ & 10.371 \\
\hline
\end{tabular}


are taken from table 2.1 .

Consider maximizing $\eta$ for $A_{3}$ (Fig 2.3). Initially $\eta=4.8$ and $\mu=1.54$, but after $300 \mathrm{FE} s, \eta=6.00$ and $\mu=4.0 \times 10^{3}$ which give an $\alpha$ inferior to that of Table 2.1. However, assuming $c_{0}$ constant in 2.1.15, initially, $\alpha=\Omega / 2-\sqrt{\mu} c_{0}=2.4-\sqrt{1.54 c_{0}}$. Taking values from Table 2.1, $\alpha=3.0-200.78 c$, which is grossly inferior. This casts some doubt on the usefulness of problem (b). The matrix $\mathrm{A}_{2}$ causes some lack in convergence as in Table 2.2. The quantity $\eta^{2} / \mu$ determines the size of the crude RAS and optimum values were superior to those calculated from Table 2.1 . Finally, the use of the algorithm seems infeasible in locating the spread $M(A)-m(A)$ by minimizing $s\left(P^{-1} Q\right)$, due to high computing time (100 times greater for $n=3$ than straight calculation of $\lambda(A)$ ) and the lack of convergence to the minima. 

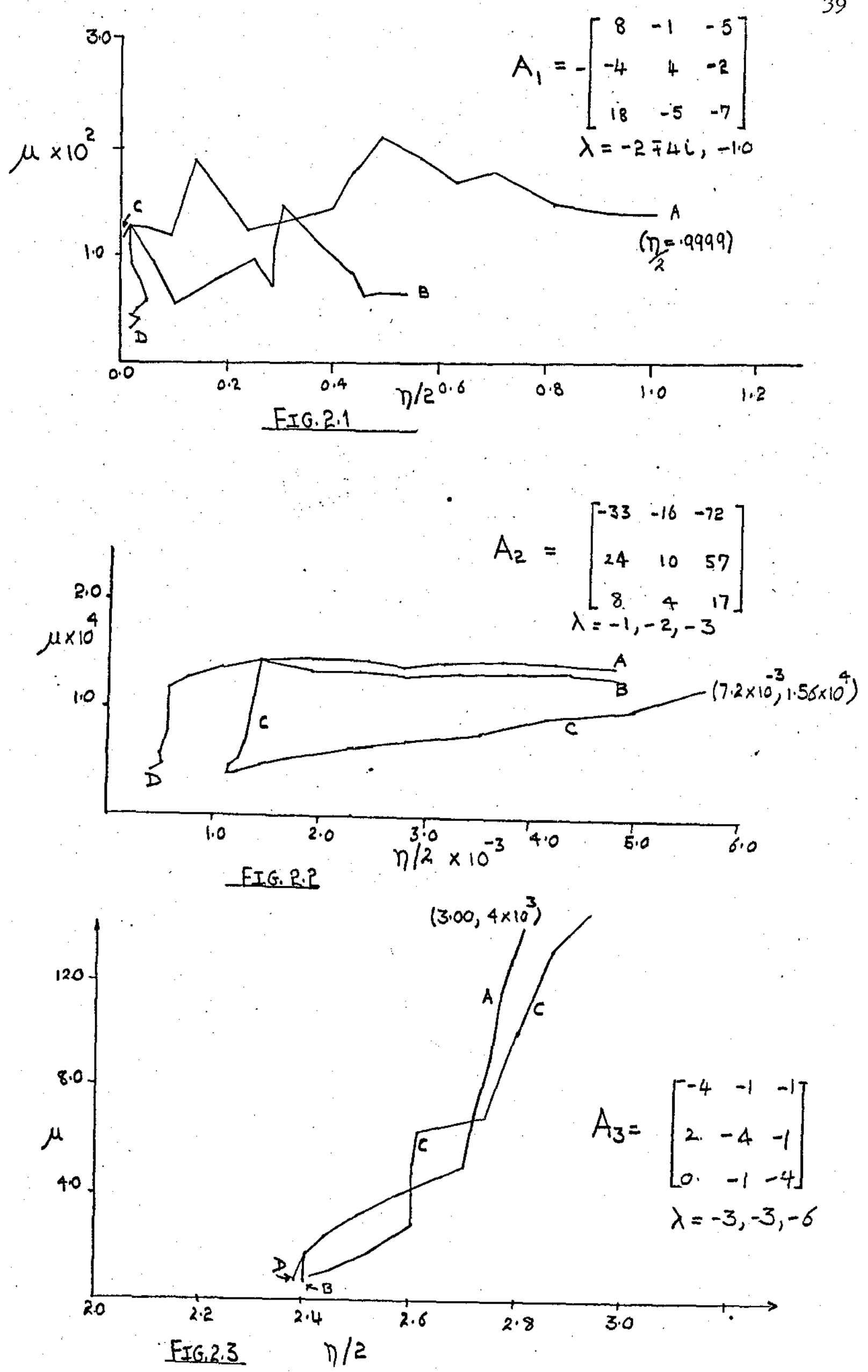
A: $\min (-n)$
$B: \min \left(-\eta^{2} / \mu\right)$
C: $\min \left[M\left(P^{-1} Q\right)-m\left(P^{-1} Q\right)\right]$
Di $\min \mu(P)$ 
CHAPTER 3.

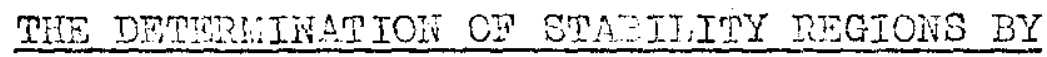
ZUBOV'S APEOONCHA 


\section{Chaver 3}

\section{The Determination of Stability Regions}

\section{by Zubov's aporoach}

\subsection{Introguction}

Although applicable to the theory of aymamic systens in seneral, the work of zubov (1) has shown greatest use in the detemination of regions of asylptotic stability (RAS) of autonomous non-inear differential equations. It reduces the choice of a Lrapunov function to the solution of a partial differential equation (PDE), the exact solution or which determines the precise D.O.A.

\subsection{The Hain Theoren}

Consider the autononous differential equation (d.e.)

$$
\underline{\dot{x}}=\underline{f}(\underline{x})
$$

where $\underline{\underline{E}}(\underline{0})=0$ and $\underline{\underline{E}} \in \mathbb{E}, \quad \underline{x} \in E^{n}$ and where $\underline{x}=0$ is an asymptotically stable equilibriun point. Then the core of zubov's treatment lies in the following theorem (1).

Theorem 3.1 .

Let $\mathrm{U}$ be an open region containing the orisin and $\vec{U}$ itis closure.

Then a necessary and sufficient conition for $U$ to be the comain of attraction (DCA) of syster 3.2.1. is the existence of two functions $W(\underline{x})$ and $\psi(\underline{x})$ with the properties:

a) $(\underline{x})$ is defined and continuous in $U$

b) $\psi(\underline{x})$ is positive definite and continuous in $E^{n}$ 
c) $0<w(\underline{x})<1 \quad$ for $\underline{x} \in U, \underline{x} \neq 0$

d) if $\mathrm{y} \in \mathrm{B}(\mathrm{B} \equiv \overline{\mathrm{U}}-\mathrm{U})$ then $\lim \mathrm{W}(\underline{\mathrm{x}})=1$ $x \rightarrow y$ and if $\|\underline{x}\| \rightarrow \infty$ for $\underline{x} \in U, \lim W(\underline{x})=1$ $\|\underline{x}\| \rightarrow \infty$

e) $\frac{d W}{d t}=\dot{W}=\sum_{i=1}^{n} \frac{\partial W_{i}}{\partial x_{i}} f_{i}=-\psi(\underline{x})(1-W(\underline{x}))\left(1+\|\underline{\underline{I}}\|^{2}\right)^{\frac{1}{2}}$ By assuming the $f_{i}$ terms are bounded the factor $\left(1+\|\underline{e}\|^{2}\right)^{\frac{1}{2}}$ in $(e)$ may bo removed giving the main PDE $\dot{W}(\underline{x})=\sum_{i=1}^{n} \frac{\partial W}{\partial x_{i}} \cdot f_{i}=-\phi(\underline{x})(1-w(\underline{x}))$

where $W$ satisfies (c), which we call the regular equation.

By defining another p.d. function

$$
V(\underline{x})=-\ln (1-W(\underline{x}))
$$

3.2.2 may be transformed into

$$
\dot{v}=\sum_{i=1}^{n} \frac{\partial v_{i}}{\partial x_{i}} f_{i}=-\phi(\underline{x})
$$

which we call the modified equation. The solutions if and $V$ of 3.2 .2 and 3.2 .3 for arbitrary $\phi(\underline{x})$ then give for the boundary of the D.O.A., B, either of the sets

$$
W(\underline{x})=1
$$$$
3.2 .4
$$

ox

$$
v(\underline{x})=\infty
$$$$
3.2 \cdot 5
$$

In what follows we concentrate on 3.2 .3 for convenience (P.D.E. 3.2.2 can be treated similarly). 


\subsection{The Construction Procedure}

We make the following assunptions:

(a) the components of $\underline{I}$ may be expanded as convercient power series about $\underline{x}=0$.

i.e.

$$
f_{i}(x)=\sum a\left(i_{1}, i_{2}, ., i_{n}\right)_{x_{1}} i_{1} x_{2} i_{2} \ldots x_{n}{ }^{i_{n}}
$$

(b) the linear part is asymptotically stable.

In all but the simplest cases the analytic solution for $\checkmark$ of 3.2 .3 is impossible. Consequently, Zubov proposed the following procedure.

Express $\mathrm{V}$ and $\phi$ as power series of arbitrary degree $m$ and I respectively, and write

$$
\begin{array}{lll}
v^{m}=v_{2}+v_{3}+\cdots & +v_{m} & 3.3 .5 \\
\phi^{1}=\phi_{2}+\phi_{3}+\cdots & +\phi_{1} & 3.3 .6
\end{array}
$$

where $v_{i}, \phi_{i}$ are homogereous polynomials of degree $i$ and $\phi_{2}$ is positive definite.

Substitute these series for $v, \phi$ and $f$ into 3.2 .3 and equate coefficients of like-powers. There then results a set of simultaveous equations for the coefficients of the powers of the $v_{i}$ terms, which may be solved successively for those of $V_{2}, V_{3}$, onwards. Since $\phi_{2}$ is p.d. $V_{2}$ is by (b) above and termination of the series, 3.3 .5 for any finite $m$ mill result in an $m$ - th degree Lyapunov function (2). An RAS, with bounciary $V^{m}=C_{m}$, can then be obtained with recourse 
to theorem 1.6.1.

A vealth of experience on the determination of stability regions, (2) - (6), has show that the sexies procedure may be non-uniformly convergent in that higher degree Iyamunov function san result in inferior RAS 's. It is difficult to show why or how this happens in general, but the following example throws some light on the issue.

\subsection{An Examnle (Zubov)}

The system is

$$
\begin{aligned}
& \dot{x}=-x+2 x^{2} y \\
& \dot{y}=-y
\end{aligned}
$$

whose D.O.A. is $x y<1$

Write 3.3 .5 as

$$
v^{m}(x, y) \quad=\sum_{i=2}^{m} \sum_{j=1}^{i+1} \text { aij } x^{i-j+1} y^{j-1}=\sum_{i=2}^{m} v_{i}
$$

and choose

$$
\phi=2\left(x^{2}+y^{2}\right)
$$

3.4 .2 .

Then substitution into $3 \cdot 2 \cdot 3$. Eives

or

$$
\frac{\partial V^{m}}{\partial x}\left(-x+2 x^{2} y\right)+\frac{\partial V^{m}}{\partial y}(-y)=-2\left(x^{2}+y^{2}\right)
$$

$$
-\sum_{i=2}^{m} i, v_{i}+2 x^{2} y \sum_{i=2}^{m} \frac{\partial v_{i}}{\partial x}=-2\left(x^{2}+y^{2}\right) \quad 3.4 \cdot 3 .
$$

Equating coeffts. of like powers and putting $u=x y$ gives the $2 m-t h$ dog. Iyapunov Function (IF), 


$$
v^{2 m}=y^{2}+x^{2}\left(1+u+u^{2} \cdot \cdot+u^{m-1}\right) \quad 3.4 \cdot 4
$$

Assuning $|u|<1$, and taling the limit, actualiy gives the analytic solution

$$
v=y^{2}+\frac{x^{2}}{1-x y}
$$

However nurierical calculation of the RAS's for various $m$ by the method of Rodden (3) gives the RAs boundaries in wis. 3.1. ( $m=51$ was near to the overelow value of $\mathrm{m}$ in computation).

The average radius $(1.14)$ for $m=50$ is slightly smaller than that for the quadratic (1.24), and the procedure is highly non-uniform. Considering the stability boundaries for $m$ odd and $m$ even seperately, We appear to have uniforn convergence in each case, those for m oda being superior. Analysing the example further indicates why this should happen. For $\mathrm{m}=2$ and $\mathrm{m}=4$ analytic calculations give the following RAS boundaries and tangency points,

$$
\begin{aligned}
& v^{4}=y^{2}+x^{2}(1+u)=\frac{4 \sqrt{3}}{9} \\
& \text { Tan. pts, }-\left(-\sqrt{\frac{2}{\sqrt{3}}}, \sqrt{\frac{2}{3 \sqrt{3}}}\right)
\end{aligned}
$$

and:

$$
v^{8}=y^{2}+x^{2}\left(1+u+u^{2}+u^{3}\right)=.93913
$$

with Tan. pts. $\mp(-1.0642,-.68486)$ 
These are also the largest closed contours and $\mathbb{V}$, the gradient of $\mathrm{V}$, vanishes at the respective tangency points.

We now show that for any even $m$ there are points where $Z v^{2 m}(x)=0$ for $u>-1$, and that the RAS boundaries Iie inside $|u|<1$.

From 3.4 .4 the gradient vanishes when

$$
\frac{\partial v^{2 m}}{\partial x}=v_{x}^{2 m}=x(2 P(u)+Q(u))=0
$$

and

$$
\frac{\partial v^{2 m}}{\partial y}=v_{y}^{2 m}=2 u+x^{4} Q(u)=0 \quad 3.4 .9
$$

where

$$
P(u)=\left(1-u^{m}\right) /(1-u)>0, u>-1 \quad 3.4 .10
$$

and

$$
Q(u)=1+2 u+3 u^{2} \cdots+(m-1) u^{m-2} .
$$

Clearly $\exists$ a $u,-1<u<0$, such that

$$
\begin{aligned}
F(u) & =2 P(u)+Q(u)=0 \\
& =2+3 u+4 u^{2}+\cdots+(m+1) u^{m-1}
\end{aligned}
$$$$
3.4 .11
$$

since $F(0)=2$ and $F(-1)<0$.

If $F(\tilde{u})=0$ then 3.4 .11 implies by 3.4 .10 that

$$
Q(\bar{u})=-2 P(\bar{u})<0
$$

Then with $u=\bar{u}$, equation 3.4 .9 vill vanish for some $x=\vec{x} \neq 0$ because this implies

$$
\overline{\mathrm{x}}^{4}=\frac{-2 \bar{u}}{\mathrm{Q}(\overline{\mathrm{u}})}=\frac{\overline{\mathrm{u}}^{2}}{\mathrm{P}(\overline{\mathrm{u}})}>0
$$

Thus, $\exists$ at least two radially symmetric points where

$$
Z V=0, \text { namely } \mp(\bar{x}, \bar{u} / \bar{x})
$$


Let $v^{2 m}(x, y)=C_{n}$ be the larsest closed RAS boundary. Then it lies in $u>-1$. For otherwise $\exists$ two distinct pts., $\left(x_{1}, y_{1}\right),\left(x_{2}, y_{2}\right)$ say, in common with $x y=-1$ and this boundary. By 3.4.4, at these points.

$$
\mathrm{v}^{2 \mathrm{~m}}\left(\mathrm{x}_{1}, \mathrm{y}_{1}\right)=\mathrm{y}_{1}^{2}=\mathrm{v}^{2 \mathrm{~m}}\left(\mathrm{x}_{2}, \mathrm{y}_{2}\right)=\mathrm{y}_{2}{ }^{2}
$$

then $y_{1}=y_{2} \Rightarrow x_{1}=x_{2}$, a contradiction. Finally the boundary lies inside $u<1$ since this is the D.O.A.

Consider now m odd. Summing the geometric series in the $u$ terms in 3.4 .4 gives

$$
v^{2 m}=y^{2}+x^{2}\left(1-u^{m}\right) /(1-u) \quad 3.4 .12
$$

the contours of which will always be closed since it is p. a. and tadialy uncounded. Hence there is no restriction to the convergence of the RAS. to the DOA for increasing in odd.

\section{Coursent}

Let $R$ be the region of convergence or the series 3.5.5 with partial sum $\mathrm{V}^{\mathrm{m}}$. We have shom an example where $R(|u|<1)$ is a subset of the DOA, where some truncations ( $m$ even) always give inferior RAS's, and where others ( $m$ odd) give better ones but converge sowly to the DOA ( $\mathrm{m}=51$, still poor?). We ask ' is convergence of the RAS to the DOA always non-uniform if $R \subset U$ ?' Ho arswer has jet been forthcoming. 


\subsection{Direct Nunerical Solution of the P.D.E.}

Due to the non-uniformity of the Zubov procedure and the infeasibility of solving the series solution for large $n(2)$, even though fomulation is possible (7), it seems reasonable to Iook for other mothode of solving 3.2.3. In this section we consider solving the P.D.E. by a finite difference method. We restrict the problem to tro dinensions. Write system $3 \cdot 2 \cdot 1$. as

$$
\begin{aligned}
& \dot{x}=f(x, y) \\
& \dot{y}=g(x, y)
\end{aligned}
$$

Then we rite the P.D.E.'s 3.2 .2 and 3.2 .3 as one equation.

$$
\frac{\partial V}{\partial x} f+\frac{\partial V}{\partial y}=-\phi+[\phi V]+3.5 .2 .
$$

where[ ] is incluaed for 3.2 .2 only, the regular equation.

From a classification point of view, 3.5 .2 is a

linear PDE solvable by the method of characteristics

Unfortunately this leads us back to the solution of the system trajectories.

ConsequentIy, we express 3.5.2 in polar co-ordinates

$$
\frac{\partial V}{\partial r} \cdot F(r, \theta)+\frac{\partial V}{\partial \theta} \cdot G(r, \theta)=\underset{3.5 .2}{-} \underset{3}{-\phi}+[\phi V]
$$

where $F=(f \cos \theta+g \sin \theta)$

$$
G=\frac{1}{r}(\dot{g} \cos \theta-r \sin \theta)
$$


Consider the mesh in Diag. 1.

where

$\mathbf{r}_{i}=i n, i=1,2, \ldots$

$\theta_{j}=(j-1) k, j=1, \ldots, N+1$

$k=2 \pi / N, \theta_{1}=\theta_{i+1}$

and $V_{i, j}=V(i h,(j-1) k)$

\section{Diag. 1}

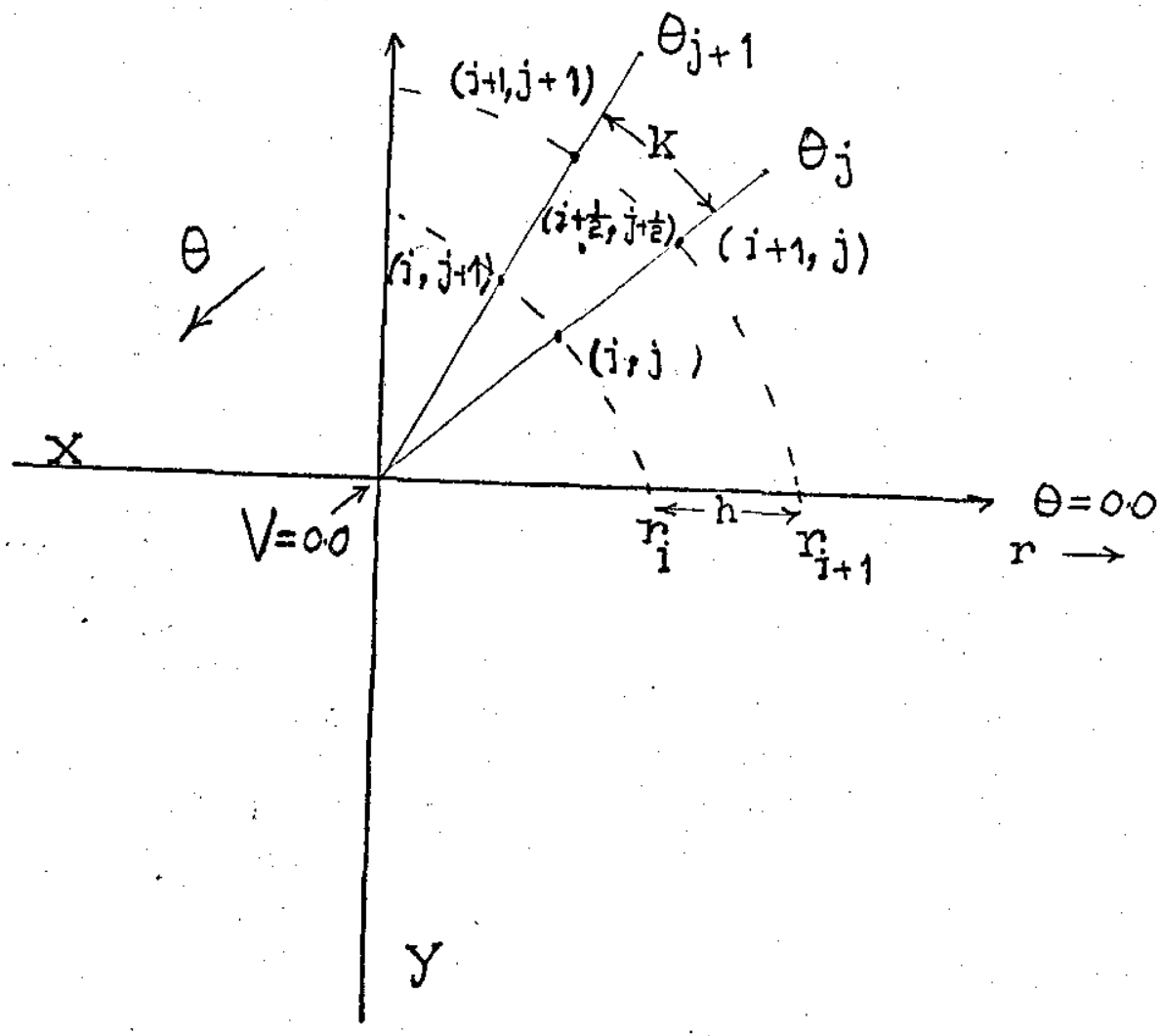


Choosing $\phi=\lambda\left(a x^{2}+2 b x y+c y^{2}\right)^{\prime}$ and makings the approximations about the mesh point $\left(i+\frac{1}{2}, j+\frac{1}{2}\right)$ $\left(\frac{\partial V}{\partial r}\right) \bumpeq \frac{1}{2 h}\left(\left(v_{i+1, j}+v_{i+1, j+1}\right)-\left(v_{i, j}+v_{i, j+1}\right)\right)+o(h, k)$ $\left(\frac{\partial V}{\partial \theta}\right) \bumpeq \frac{1}{2 i \Sigma}\left(\left(v_{i, j+1}+v_{i+1, j+1}\right)-\left(v_{i, j}+v_{i+1, j}\right)\right)+o(k, h)$ substitution into 3.5 .3 gives the following CrankNicholson type difference equations (9) on neglecting second order terms in $h$ and $k$,

$$
v_{i+1, j^{a}}+v_{i+1, j+1} b_{j}=c_{j} \quad 3.5 .6
$$

where $\quad v_{N+1}=v_{1}$

and

$$
\begin{array}{ll}
a_{j}=F-p G-z & 3.5 .7 \\
b_{j}=F+p G-z & 3.5 .8 \\
c_{j}=v_{i, j}\left(b_{j}+[2 z]\right)+v_{i, j+1}\left(a_{j}+[2 z]\right)-4 z \\
p=h / k, \quad z=h \phi / 2
\end{array}
$$

The terms in [] are zero for the modified egn. ( $\phi, F$ and $G$ are evaluated at $\left(i+\frac{1}{2}, j+\frac{1}{2}\right)$ in 3.5 .7 and 3.5 .8$)$. Since $\mathrm{V}(0,0)=0$, we can assume

$$
v\left(r_{1}, \theta_{j}\right)=V(h, j k)=\epsilon, \quad j=1, \ldots, N \quad 3.5 .10
$$

for initial conditions $(\epsilon>0$, small $)$, or, make

$$
v(h, j k)=v_{2}(h, j k) \quad j=1, \ldots, N
$$

$\mathrm{v}_{2}$ being obtained fron the series 3.3.5.

We have an initial value problem (9) and by writing 3.5 .6 as a linear system of equations

$$
\mathrm{AV}_{\mathrm{i}+1}=\mathrm{c}
$$


where $c=\left(c_{1}, c_{2}, \quad, c_{N}\right)$,

$$
V_{i+1}=\left(v_{i+1,1}, \ldots \ldots . v_{i+1, N}\right)
$$

and $A$ anif $x$ coefficient matric, an efficient conputer algorithm results for treir solution (A2).

\subsection{Numerical Exarnles}

The following examples show features of the method.

Exarinle 3.6.1

The system is.

$$
\begin{aligned}
z & =-x+y+x\left(x^{2}+y^{2}\right) \\
\because \quad \dot{y} & =-y-x+y\left(x^{2}+y^{2}\right) \\
\text { With } \phi & =2 x^{2}, N=100, n=.0125, r_{1}=.25
\end{aligned}
$$

Solution of the rezular equation gave $V=1.0000$ for all mesh points on $r=1.0$. The analytic solution is $V=r^{2}$ giving $r^{2}=1.0$. for $B$. Witr $\phi$ changed to $\phi=10 x^{2}+y^{2}, V=1.0000$ again on $\left(1 \cdot 0, e_{j}\right)$. Constructing the $V$ contours shows they are no longer concentric circles as above but ellipses, approaching $x^{2}=1$ as $x \rightarrow 1$. Fig. 3.2 shows variation of $V$. With $\theta$ for fixed $r$. Note how the numerical solution of $\mathrm{V}$ becomes negative for $r>1$, indicating that the analytic solution is not defined for sucin $r$. Exarane 3.62 .

A Van dor Pol equation.

$\dot{x}=y$

$\dot{y}=-x-y+x^{2} y$

Fir. 3.5 shoms attempted constructions of some $V$ contours from mesh values, for the regular equation, fith $h=.0125$, 
$r_{1}=25, \phi=r^{2}$, and $n=100$. Singularitics

occured at the DOA boundary, $B$, as in 3.6.1, witis $\mathrm{V}$

taking on large negative values $(i \rightarrow-\infty)$

Choice of nesh was important since

$s=y=0$ for $\theta=0$. If $\theta_{j+\frac{j}{2}}=0$ for some $j$,

the method breaks down.

\section{Examp le 2.5.3}

The system is $3 \cdot 4 \cdot 1 \cdot$ with $\phi=2\left(x^{2}+2 x y+2 y^{2}\right)$

and initialiy $v\left(r_{1}, \theta\right)=v_{2}=\phi / 2, r_{2}=.0125$

$r_{1}=-25$ and $N=100$, errors between the numerical

and analytic results were less than $10^{-4}$ for the refrular equation, for $x<1 \cdot 375$. As $r \rightarrow 1 \cdot l_{\text {, the }}$ $\mathrm{V}_{1}, \mathrm{y}_{\mathrm{j}} \rightarrow-\infty$ renderine computed values useless.

The reason is found in the analytic solution.

$V=1-e^{-\left(y^{2}+x^{2} /(1-x y)\right)}$

since $\mathrm{V} \longrightarrow-\infty$ as $\mathrm{xy} \longrightarrow 1+$

Fig. 3.3 shows a construction of the $V$ contours, while Fig. 3.4 , the variation of $V$ along a ray to $B$ for various $\lambda$

\section{Comment}

If numerical values are correct the mesh points at which $V_{i, j}<1$ lie in $U$, the DOA. Unfortunately the method breaks down near the DOA boundary and the values at such mesh points, $v_{i, j}$, are such that $v_{i, j} \rightarrow-\infty$. The accuracy of other points where $v_{i, j}<1$ is then decreased. 
Suitable choice of $\lambda$ is also a problem. Large values of $\lambda$ means $v \rightarrow 1$ guickly (Mnalytically, if $V$ solves $\dot{V}=-\phi$ then $\hat{V}=\lambda v$ solves $\dot{\hat{V}}=-\lambda \phi)$ and conversely for $\lambda$ mall (rig.3.4). ivumericaliy, a criterion such as

$$
0<1-v_{i, j}<10^{-t}
$$

gives a point 'near' $D$. In gereraj. $t$ will cepend on $\lambda$. Eirally, we add that other differerce schemes for solving 3.5 .3 have given similar results.

\subsection{Recent hethods}

It is pertinent in view of section 3.6 to mention other nethods of solving Zubov's PDI 3.2.2/3.2.3. Eumand and Sarlos (1968) (10), treat 3.2.3 and syster 3.2 .1 as two differential equations. Using Lie series, the two equations in question,

$$
\begin{array}{ll}
\dot{z}=x(x) & 3.7 .1 . \\
\dot{r}=-\phi(x) & 3.7 .2 .
\end{array}
$$

with $\phi p$. dor., are integrated wi th reverse time from an initial point $\underline{x}(0)$ near the origin (typically $\mathrm{V}(\mathrm{x}(0))=0.0$ ) until $\mathrm{V}>\mathrm{K}$, where $\mathrm{K}$ is a lange positive number $\left(e . \tilde{s} \cdot F_{=}=20\right)$. Hopefully the final point $x(t)$ is near $\mathrm{D}$. The proceduro is repeated for a series of initial points untic i is traced out. Formanit and Ii (1972) (13) have ertended the nethod and fit an alfobraic curve to the rinal set of points by a pattern classification alsorithr. 
Troch (1972) (12), with conoutation efficiency in mindinterrates systen 3.7 .1 and $3.7 .2 \mathrm{bJ}$ analogue conputer. The toruination criterion $V>K$ cetermines a point on $\mathrm{D}$ ana a more accurate oicitaz computation interrates a trajectory thirugin tinis point. The trajectory (or a series of such) will trace out $\mathbf{B}$.

\section{Commont}

From Iinited computer experience we founa burnand Sarios's metnod suffered from two drawbacks:

a) although accurate, cormuter time was high for the Lie series computation,

b) caiculation of the recursive terms (10) in the Lie series ras infeasible for complex r.r.s. of 3.7 .1$.

As a criticism of both inethods we ask, is the aciditional solution of 3.7 .2 realiy necessary since the criterion $V>k$ (or $\cdot 1-V<\epsilon$, where $V=1-0^{-V}$ ) is scownat arbitrary as a criterion for 'nearness' to B? The non Iyapunov method of Davidson and Cowan (1969) (11), restricted to $n=2$, gives a partial answer. For their criterion they use the function

$$
V\left(\underline{x}\left(t_{0}\right)\right)=\| \underline{X}\left(t_{0}\|-\| \underline{X}\left(t_{0}+\underline{T}\right) \|\right.
$$

where $T$ is the pericuic time (ror Iimit cycle) or an upper bound (for nocal type systerns). Equation 3.7.1 is interrated by a fourth orcer Runce Kutta formula and 
a point satisfying $V(\underline{x}(t))=0$ determines

a point on $D(13)$.

Finsily, it is questionable whether any of the arove

metiocis are useful for $\mathrm{n}>2$ in view of the computation tine involved. 


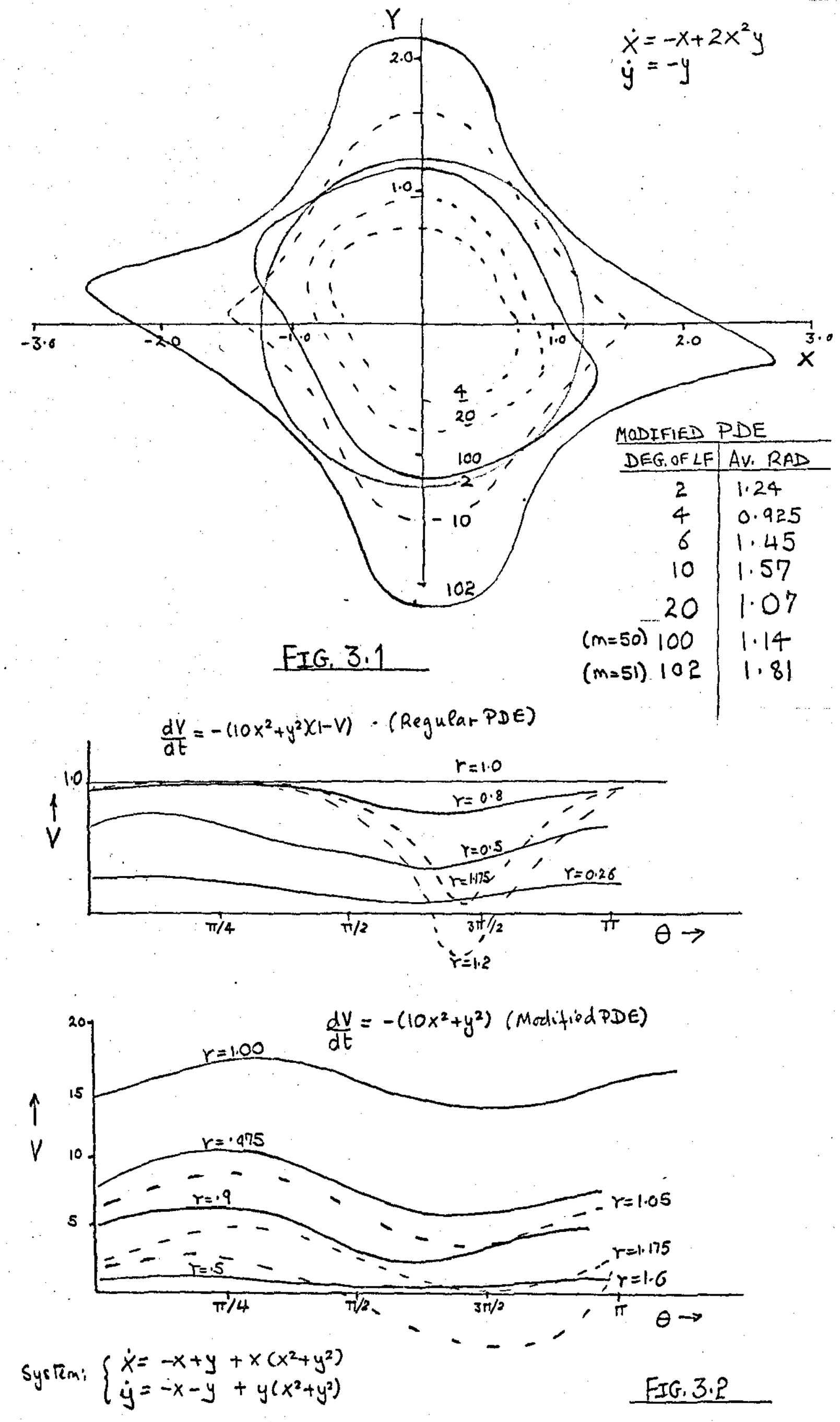


56

VARIATION OF $V$ ALONG RAY $\theta=41^{\circ} 40^{\prime}$ FOR VARIOUS $\lambda$.

REGULAR DE $\frac{d v}{d t}=-\phi(1-v)$

SYSTEM:

$$
\begin{aligned}
& \dot{x}=-x+2 x^{2} y \\
& \dot{y}=-y
\end{aligned}
$$
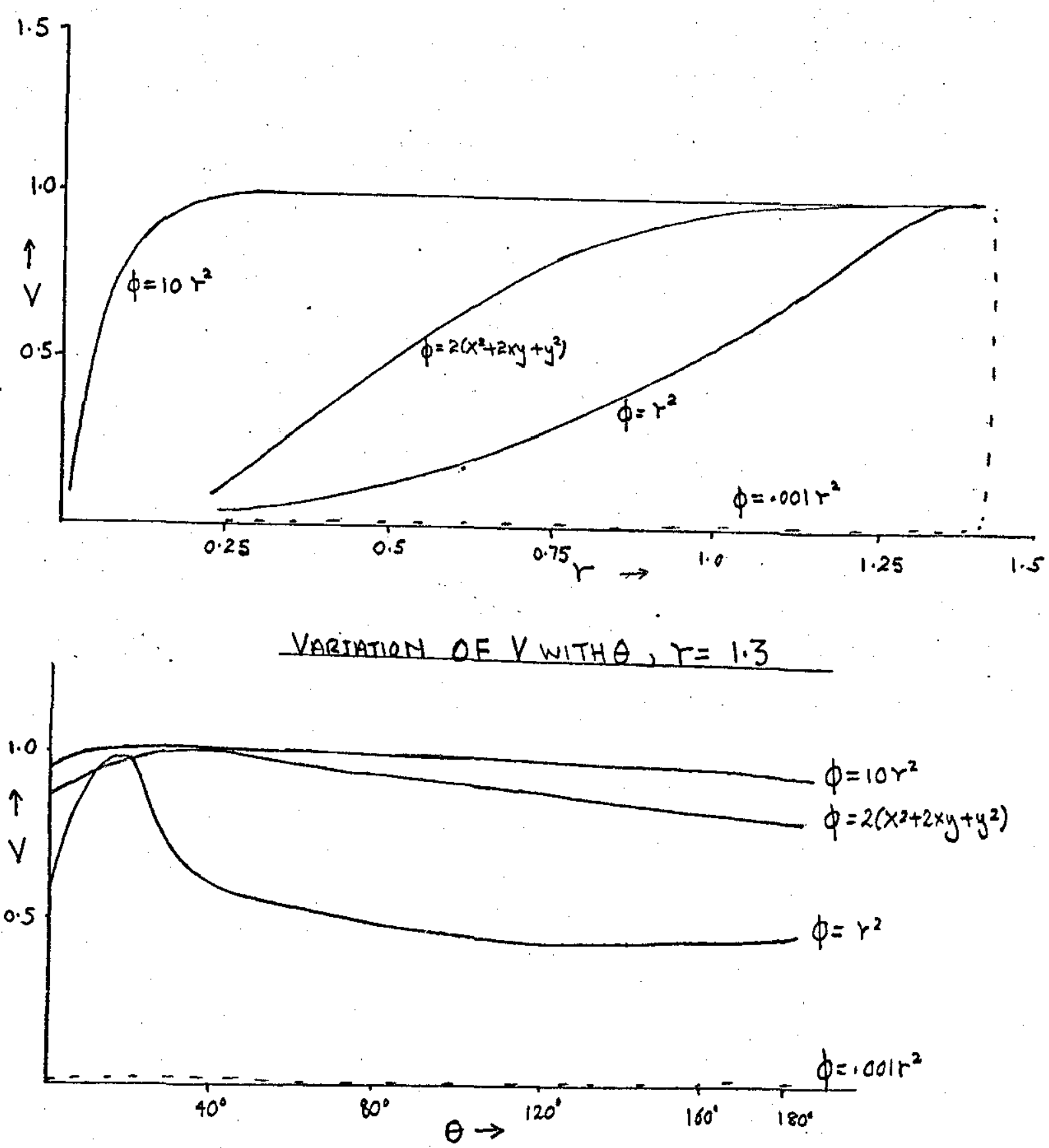

FIG. $3 \cdot 4$ 


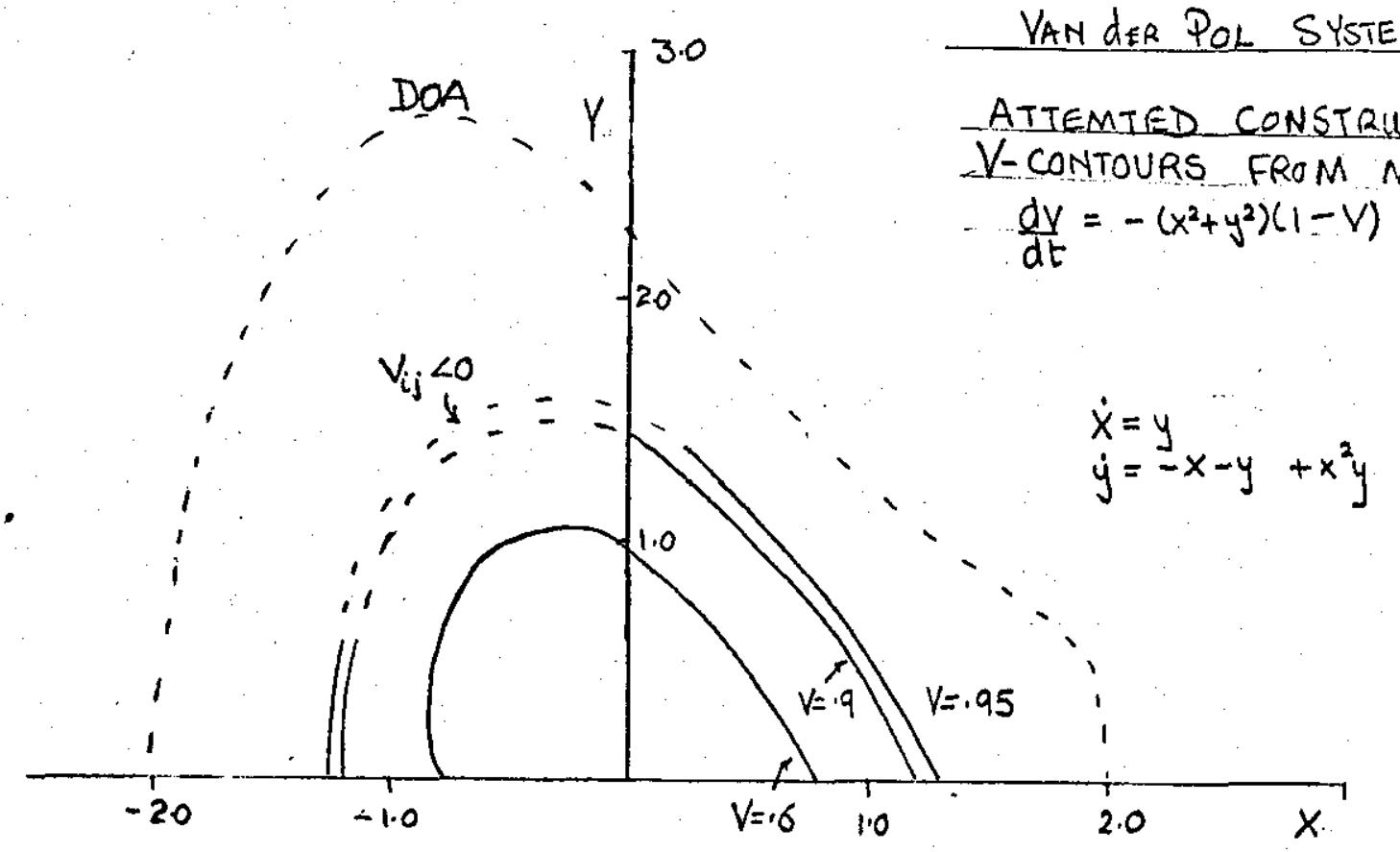

FIG 3.5

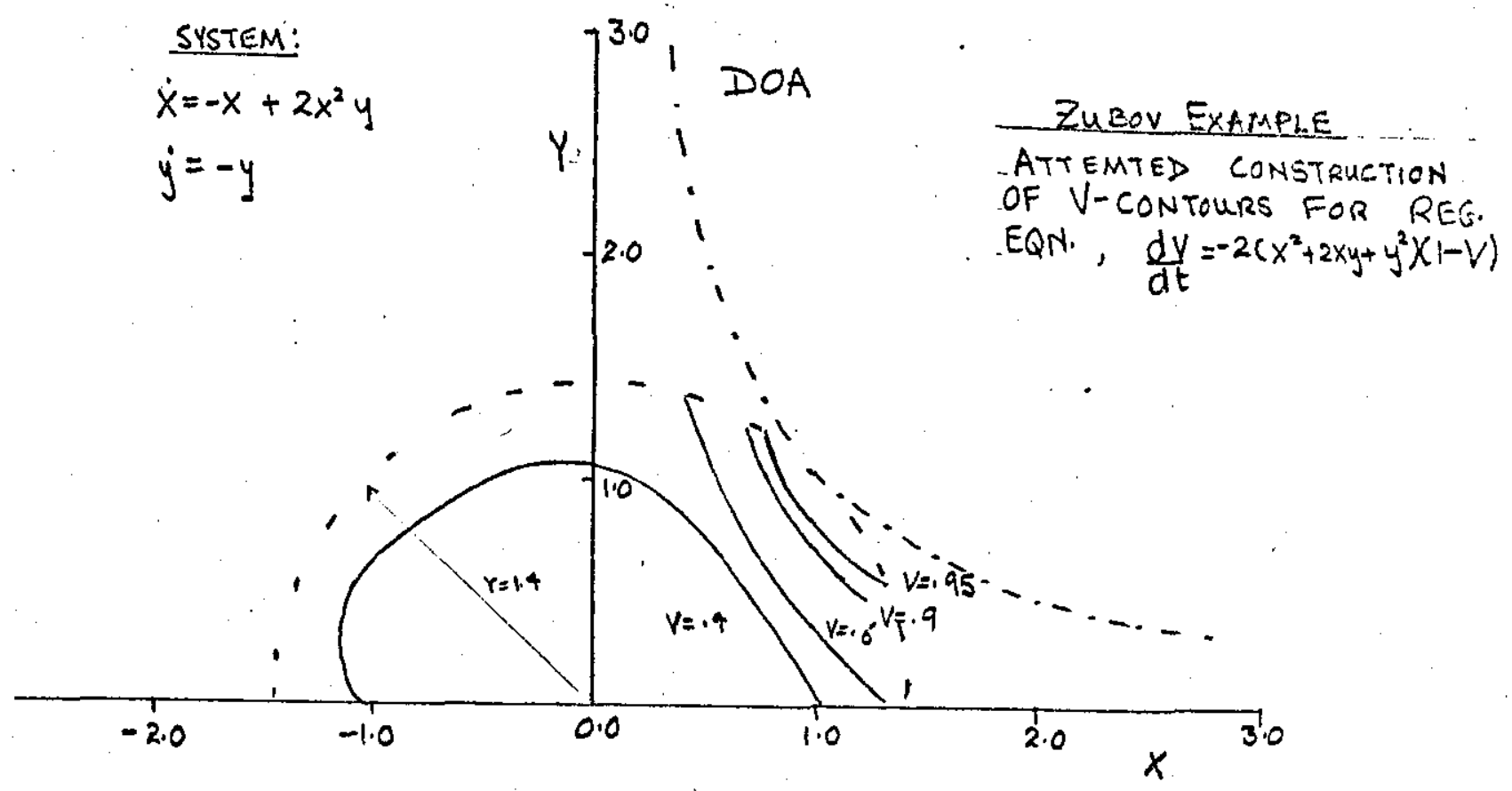

FIG. 3.3 
CHAPTER 4

OPTIMAL QUADRATIC LYAPUNOV FUNCTIONS 


\section{CHAPTER 4}

\section{Optimal Quadratic Lyapunov Functions}

\subsection{Introduction}

We will be concerned with the autonomous system 1.4 .3 name Iy,

$$
\dot{x}=A \underline{x}+g(\underline{x})
$$

where $A$ is stable and $B$ is expandable as a power series with terms of at least degree two in $x_{1}$, the coefficients of $x$

A quadratic IF for $4.1 .1, V(\underline{x})=x^{T}{ }_{P}$, is determined via Theorem 1.6.1 whose RAS is given by

$$
\underline{x^{T}} P \underline{x}<V_{m}
$$

where

$$
V_{m}=\min _{x} V(\underline{x}) \text { with } \underline{x} \in E_{V}
$$

Here

$$
E_{V}:\left(x / \dot{N}=-x^{T} Q x+2 x^{T} P g(x)=0, x \neq 0\right) \quad 4.1 .4
$$

and $Q$ is any p.a.s. matrix such that

$$
\mathrm{A}^{\mathrm{T}} \mathrm{P}+\mathrm{PA}=-\mathrm{Q} \quad 4 \cdot 1 \cdot 5
$$

Let $\rho(V)$ be a measure of the size of the RAS, 4.1.2.

Then a problem inherent in this chapter is to maximize $\rho(v)$ over the class of quadratic LF's,

$$
\max _{Q} \rho(V)
$$$$
4 \cdot 1 \cdot 6
$$

subject to

$$
Q>0(Q p . d . s)
$$$$
4.1 .7
$$

( $\rho$ will be some function of $P$ and this $Q ; p=\rho(P)=\rho(Q)$ ) The two usual choices of $\rho$ are the generalized volume, which for an ellipsoidal region is 


$$
\rho(P)=\pi v_{m}^{n / 2} / Q(P)^{\frac{1}{2}}, \quad 4.1 .8
$$

and the numerical average radius

$$
\rho(P)=\left(\sum_{i=1}^{N} Z_{i}\right) / N
$$

where the $\mathrm{Z}_{i}$ are points (usually equally spaced) lying on the RAS boundary. i.e. $V\left(y_{i}\right)=V_{m}$. (For a general $I F$ we have $\rho(v)=\int_{S} w(\underline{x}) d v$, a general volume measure with $s$ the RAS, $w(x)$ a weighting factor and $d v$ a volume element).

The optimum problem of 4.1.6. is highly nonlinear due to the associated RAS determination and researchers have therefore concentrated on the numerical side of its solution. The works of Weissenberger (48), Geiss (51), Szego (52) and Lapidus (49) have this emphasis and depend upon the formulation of 4.1 .3 as variants of the constrained minimization problem

$$
\min _{x \in 0} V(x)=v_{m}
$$

subject to

$$
\dot{V}(x)=0
$$

They chose various numerical optimization techniques to solve the problem.

The following sections of this chapter fill a need

in that for various systems either an optimal quadratic is found analytically or some useful RAS is determined. Some new properties are found which are confirmed to hold for general and higher order systems by efficient numerical algorithms. 


\subsection{Examples on the Determination of Optimal Quadratics and} Simple RAis's

It is well known that necessary conditions for a solution of the constrained problem 4.1.10 are the $(n+1)$ Lagrange equations in the coefficients $x_{i}$ and the Lagrange multiplier $\lambda$

$$
\begin{array}{rll}
\nabla V(x)+\lambda_{\nabla \dot{V}}(\underline{x}) & =0 & 4.2 .1 \\
\dot{V}(x) & =0 & 4.2 .2
\end{array}
$$

Geometrically, if $x^{*}$ and $\lambda^{*}$ satisfy these equations then $\nabla V\left(\underline{x}^{*}\right)=-\lambda^{*} \underline{V} \dot{V}\left(\underline{x}^{*}\right)$, which implies that the contours $V(\underline{x})=V\left(\underline{x}^{*}\right)$ and $\dot{V}(\underline{x})=0$ are 'tangential" at $x=x^{*}$. Consequently, 4.2.1 will be called the tangency equation and $x^{*}$ a tangency point. of all such $\underline{x}^{*}, x^{*} \neq 0$, we require the one minimizing $V$ on $4.2 .2 .$, i.e. the global minimum. This will be called the valid tangency point (In some cases no valid tangency exists e.g. $\forall<0, x \neq 0$; then $p$ is unbounded and $E^{n}$ the DOA) The equations 4.2 .1 and 4.2.2 also hold for a general LF but valid tangency points may exist for which $\nabla V\left(\underline{x}^{*}\right)=0$.

Analytically, 4.2.1. implies the $n-1$ independent equations

$$
\frac{v_{x_{1}}}{\dot{v}_{x_{1}}}=\frac{v_{x_{2}}}{\dot{v}_{x_{2}}}=\cdot \cdot \frac{v_{x_{n}}}{\dot{v}_{x_{n}}}
$$

with

$$
v_{x_{1}}=\frac{\partial v}{\partial x_{1}}
$$

For descriptive convenience we call the contour $\dot{v}(\underline{x})=0, x \neq 0$, (i.e. $E_{v}$ ) the constraint contour and as its 
components we use the intuitive definition as the subsets of $E_{V}$ which form continuous connected curres $(n=2)$ or surfaces $(n=3)$. If two components of $\mathrm{E}_{V}$ are radially symmetric (RS) we will call this one RS component and likewise for tangency points. In the examples which follow we choose pas the volume (or area) measure.

Example 4.2 .1

Consider the system from Zubov (1) in 3.4.1, nanely,

$$
\begin{aligned}
& \dot{x}_{1}=-x_{1}+2 x_{1}{ }^{2} x_{2} \\
& \dot{x}_{2}=-x_{2}
\end{aligned}
$$

with DOA $x_{1} x_{2}<1$. Any p.d. quadratic is a IF for this system so let $V=a x_{1}^{2}+2 b x_{1} x_{2}+c x_{2}^{2}$ with $a>0, a c-b^{2}>0$. Then 4.2 .2 gives

$$
\dot{V}=2\left(-V+2 x_{1}^{2} x_{2}\left(a x_{1}+b x_{2}\right)\right)=0 \quad 4.2 .4
$$

and after some manipulation 4.2 .3 gives

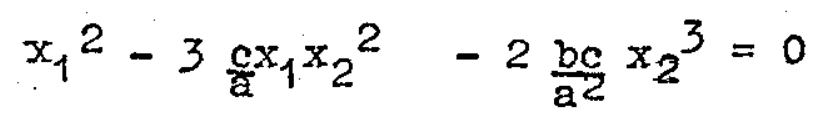

Let $u=x_{1} / x_{2}$, then dividing 4.2 .5 by $x_{2}{ }^{3}$ gives the tangency equation

$$
u^{3}-3 \frac{c u}{a}-2 \frac{b c}{a^{2}}=0 \quad 4: 2.6
$$

It is well known that the standard cublc $u^{3}+p u^{+} q=0$ has real roots if $p<0$ and $4 p^{3}+27 q^{2}<0$. In this case they are given by

$$
u=x_{1} / x_{2}=2 \sqrt{\frac{c}{a}} \cos (1) \quad 4.2 .8
$$

where $1=\frac{1}{3} \cos ^{-1}(z), \quad I=\frac{1}{3}\left(2 \pi+\cos ^{-1}(z)\right)$ 
with $z=b / \sqrt{a c}$. Substituting 4.28 into 424 gives

$$
\bar{x}_{2}{ }^{2}=(a / c) \frac{\frac{1}{2} z\left(4 \cos ^{2}(1)+4 z \cos (1)+1\right)}{8 \cos ^{2}(1)(2 \cos (1)+z)}
$$

and thus

$$
V_{m}=\frac{(a c)^{\frac{1}{2}}\left(4 \cos ^{2}(1)+4 z \cos (1)+1\right)^{2}}{8 \cos ^{2}(1)(2 \cos (1)+z)} \quad 4.2 .10
$$

The $\operatorname{area} \rho(V)=\rho(a, b, c)$ of the RAS is

$$
\rho=\frac{\pi}{8} \frac{\left(4 \cos ^{2}(1)+4 z \cos (1)+1\right)^{2}}{\sqrt{1-z^{2}} \cos ^{2}(1)(2 \cos (1)+z)} \quad 4.2 .11
$$

which, interestingly, is a function of one parameter, $z$.

For p.d. V we reguire $|z|<1$ and a>0. Given such $a z$, by varying $a, b$ and $c$ to satisfy $z=b /(a c)^{\frac{1}{2}}$, an infinite number of quadratics exist all giving the same area for $p$. Since this is true for the optimizing $z, z^{*}$ say, the optimal quadratic is in this sense non unique. It can be shown that $z^{*}=1 / \sqrt{ } 2$ giving $\rho=4 \pi$.

By inspection of 4.2 .4 the lines $x_{1}=0, x_{2}=0$ and. $a x_{1}+b x_{2}=0$ separate the phase space into regions where $R S$ components for $E_{v}$ lie. Further, we have for

a) $z \leq 0,1$ RS component and one tangency point.

b) $z>0,2$ RS components and 2 tangency points. (See Fig. 4.1). Also $\rho$ is small for $z<0$ and increases to $4 \pi$ with increasing $z$ until $z=z^{*}$, thereafter decreasing. The valid tangency point for $0<z</ \sqrt{2}$ lies on a $R S$ component in $x_{1} x_{2}>0$, whereas for $\frac{1}{\sqrt{2}}<z<1$, on a RS component in $x_{1} x_{2}<0$, When $z=z^{*}=1 / \sqrt{2}$ we have the important property that two valid tangency points exist and thus an optimal quadratic boundary $V=V_{m}$ touches both $R S$ components. 
The re are an infinitenumber of such boundaries satisfying $\sqrt{2} b=\sqrt{a c}$. Which, in the limit, sweep out an open region. Fig. 4.2 shows some of these together with two loci obtained by eliminating the variable quantity $(a / c)^{\frac{1}{2}}$ between 4.2 .8 and 4.2 .9 giving a locus of tangency points as

$$
x_{1} x_{2}=\frac{\left(4 \cos ^{2}(1)+4 z \cos (1)+1\right)}{\cos (1)(2 \cos (1)+z)}
$$

For $z=z^{*}$, the two divalues of 1 are $I_{1}=\pi / 12$ and $I_{2}=7 \pi / 12$ which give the loci $x_{1} x_{2}=\sqrt{3}-1$ and $x_{1} x_{2}=-(\sqrt{3}+1)$ respectively. Since a quadratic RAS is a convex region an estimate of the DOA is given by points satisfying $x_{1} x_{2}>-\sqrt{3}-1$ and $x_{1} x_{2}<\sqrt{3}-1$. A better estimate are the loci of extreme points on the major and minor axes given by $\left(R_{1} \cos \theta_{1}, R_{1} \sin \theta_{1}\right)$ and $\left(R_{2} \cos \theta_{2}, R_{2} \sin \theta_{2}\right)$ where for $b>0$ $R_{1}=\sqrt{\frac{2}{b^{3}}\left(2 b^{2}+1+\sqrt{1+4 b^{2}}\right)}, \quad R_{2}=\sqrt{\frac{2}{b^{3}}\left(2 b^{2}+1-\sqrt{1+4 b^{2}}\right)}$ and $\theta_{1}=(\pi+a) / 2, \quad \theta_{2}=a / 2, \quad a=\tan ^{-1}\left(2 b /\left(1-2 b^{2}\right)\right)$

\section{A System of Special Form}

A simple quadratic RAS may be determined for a second order system of the form 4.1.1. With $g(x)$ an homogeneous polynomial and $A$ of the form

$$
A=\left[\begin{array}{cc}
0 & 1 \\
-\alpha & -\beta
\end{array}\right] \text { with } \beta^{2}-4 \alpha<0 \quad(\alpha, \beta>0)
$$

For then a p.d.s. $P$ exists such that ${ }_{A}^{T} P+P A=-\lambda P$, namely, to a constant factor

$$
P=\left[\begin{array}{cc}
\alpha & \beta / 2 \\
\beta / 2 & 1
\end{array}\right], \lambda=\beta
$$


Equations 4.2 .2 and the tangency equation then give

$$
\dot{V}=-\lambda v+2 \underline{x} \mathbb{E}(\underline{x}) \quad 4.2 .14
$$

and

$$
\frac{v_{x_{1}}}{v_{x_{2}}}=\frac{\left(x^{T P g}\right) x_{1}}{\left(x^{T} P_{2}\right) x_{2}}
$$

The significance is that 4.2 .15 reduces to an homogeneous polynomial in $x_{1}$ and $x_{2}$ as in 4.2 .5 with reduction to a polynomial in $\left(\frac{x_{1}}{x_{2}}\right)$. Its roots determine straight lines as in 4.2.8, and tangency points exist where such lines intersect the constraint contour, $\dot{V}=0$. An example is the Van der Pol equation:

\section{Example 4.2.2.}

The system may be written

$$
\begin{aligned}
& \dot{x}_{1}=x_{2} \\
& \dot{x}_{2}=-\epsilon\left(1-x_{1}^{2}\right) x_{2}-x_{1} .
\end{aligned}
$$

Its DOA isalimit cycle region depending upon $\epsilon>0$. Choosing $P$ in $4.2 .13,4.2 .14$ and 4.2 .15 reduce to

$$
\ddot{x}_{1}^{2} x_{2}\left(x_{1}+2 x_{2}\right)-\left(x_{1}{ }^{2}+\epsilon x_{1} x_{2}+x_{2}^{2}\right)=0 \quad 4.2 .16
$$

and

$$
\epsilon x_{1}{ }^{3}+x_{1}^{2} x_{2}\left(4-\epsilon^{2}\right)-3 \epsilon x_{1} x_{2}^{2}-4 x_{2}^{3}=0 \quad 4.2 .17
$$

where $V=x_{1}{ }^{2}+E x_{1} x_{2}+x_{2}{ }^{2}$. Substituting $z=x_{1} / x_{2}+\frac{R}{3}$

$(R=4 / E-\epsilon)$ in 4.2 .17 gives the standard cubic

$$
\mathrm{z}^{3}+\mathrm{pz}+\mathrm{q}=0
$$

For real roots $4 p^{3}+27 q^{2}<0$ which holds when $\epsilon<2$ (here $\beta^{2}-4 \alpha=\epsilon^{2}-4$ ). The roots are given as 


$$
x_{1}=x_{2}\left(2 \cos (I) \sqrt{9+R^{2}}-R\right) / 3
$$

Where $I=\frac{9}{3} \cos ^{-1}(T)$ or $I=\frac{1}{3}\left(2 \pi \mp \cos ^{-1}(T)\right)$

and $T=-\frac{\left(2 R^{3}-27 \epsilon\right)}{2\left(9+R^{2}\right)^{3 / 2}}$

It appears there are 2 RS components of $\mathrm{E}_{V}$ for a given $\in$ and only two possible RS tangeney points (or values of 1 ).

Substitution of 4.2 .18 into 4.2 .16 gives at tangency

$$
V_{m}=\frac{\left(a^{2}+\epsilon a+1\right)^{2}}{a(\epsilon a+2)}
$$

with $d=\frac{1}{3}\left(2 \cos (1)\left(9+R^{2}\right)^{\frac{1}{2}}-R\right)$. Valid values of $I$ must give $d(\epsilon d+2)>0$. When $\epsilon=1$, for example, two values of $I$ are $57^{\circ} 44^{\prime}$ and $62^{\circ} 16^{\prime}$ giving $V_{m}=1.74$, the valia boundary value, and $V_{\mathrm{m}}=2.14$ respectively.

The optimum quadratic is impossible to obtain analytically and is found numerically. (See fig. 4.5(a) and $4.5(b))$

The RAS is a reasonable estimate of the DOA.

\section{Example 4.2.2.}

Another practical example is the Duffing equation

$$
\begin{aligned}
& \dot{x}_{1}=x_{2} \\
& \dot{x}_{2}=-\alpha x_{1}-\beta x_{2}+\epsilon x_{1} 3
\end{aligned}
$$

with $\alpha, \beta_{1} \varepsilon>0$. The singular points are $P_{1}(0,0)$ and $P_{2}$ $\left(\sqrt{\frac{\alpha}{\epsilon}}, 0\right), P_{3}\left(-\sqrt{\frac{\alpha}{\varepsilon}}, 0\right)$

Consider the particular case $\alpha=\beta=\epsilon=1.0$. By choosing

$$
Q=2 \cdot\left[\begin{array}{ll}
a & b \\
b & c . l
\end{array}\right]
$$

in 4.1.5. With $Q$ p.d a general quadratic IF is found to be

$$
V=R x_{1}{ }^{2}+2 a x_{1} x_{2}+(a+c) x_{2}{ }^{2}
$$


with $R=2(a-b)+c$. The constraint and tangency equatIons give

$$
\dot{v}=2\left[-\left(a x_{1} 2+2 b x_{1} x_{2}+c x_{2}{ }^{2}\right)+x_{1}^{3}\left(a x_{1}+x_{2}(a+c)\right)\right]=0.4 \cdot 2 \cdot 20
$$

and

$$
\begin{aligned}
& -2\left[x_{1}{ }^{2}\left(R b-a^{2}\right)+x_{1} x_{2}(R c-a(a+c))+x_{2}{ }^{2}(a c-b(a+c))\right] \\
& +x_{1}{ }^{2}\left[x_{1}{ }^{2}\left(R(a+c)-4 a^{2}\right)-x_{1} x_{2} 6 a(a+c)-3 x_{2}{ }^{2}(a+c)^{2}\right]=0 \\
&
\end{aligned}
$$

As a first estimate, 4.2.14 and 4.2.15 give us, with $V=x_{1} 2+x_{1} x_{2}+x_{2}{ }^{2}$,

$$
\dot{v}=-v+x_{1} 3\left(x_{1}+2 x_{2}\right) \quad 4.2 .22
$$

and

$$
x_{2}\left(x_{1}+x_{2}\right)=0
$$

The only RS tangency point corresponds to $\mathrm{x}_{2}=0$ in the latter i.e. $\mp(1,0,0)$. It gives as RAS $V<1$, owith $\rho=$ $2 \pi / \sqrt{3}$.

Trying various values of $a, b$, and $c$ and solving 4.2.20 and 4.2.21 for the RS tangency points - which involves the real roots of a 4 th degree polynomial - it is found that (a) only one RS component of $\mathrm{E}_{\mathrm{V}}$ and one RS tangency point exists and that $(b) \rho(P)$ increases when $a=$ const. and b, $c \rightarrow 0$. In the limit 4.2 .20 and 4.2 .21 give with $v=a\left(2 x_{1} 2+2 x_{1} x_{2}+x_{2}^{2}\right)$,

$$
\dot{\nabla}=\operatorname{ax}_{1} 2\left(x_{1}{ }^{2}+x_{1} x_{2}-1\right)=0
$$

and

$$
a^{2}\left[2 x_{1}\left(x_{1}+x_{2}\right)-x_{1}^{2}\left(2 x_{1} 2+6 x_{1} x_{2}+3 x_{2} 2\right)\right]=0
$$


Clearly, $x_{1}=0$ is not a trajectory of the system except for $\underline{x}=0$.

Thus theorem 1.5.3. may be used to give an RAS. From 4.2.24 $x_{2}=\frac{1-x_{1} 2}{x_{1}}$ and use in the last equation gives F $(1,0)$ as the valid RS tangency point with $\rho(P)=2 \pi$ and with RAS boundary $2 x_{1} 2+2 x_{1} x_{2}+x_{2}^{2}=2.0$. (Numerical worls confirms that this is the unique ontimal boundary). Fig. 4.3 shows the boundary in relation to its constraint contour.

The example gives as an optimizing Q

$$
Q=\left[\begin{array}{ll}
1 & 0 \\
0 & 0
\end{array}\right]
$$

which is p.s.d. Using the same $Q$ a simple RAS boundary is easily obtained for the general case of system $S 3$ as

$$
v=x_{1}^{2}\left(\alpha+\beta^{2}\right)+2 x_{1} x_{2}+x_{2}^{2}=\frac{2 \beta \alpha}{\epsilon}
$$

with $\rho=2 \alpha \pi \beta / \epsilon$. Thus we see that increasing $\beta$, the damping coefficient, will increase the area of stability, as will decreasing $\epsilon$ the forcing term coefficient.

Example 4.2.4 (The n-Dimensional Case)

System $S 3$ is a particular case of the $n-d i m e n s i o n a l$

$$
\underline{x}=\left[\begin{array}{cccccc}
0 & 1 & 0 & \cdot & \cdot & 0 \\
0 & 0 & 1 & \cdot & \cdot & 0 \\
\cdot & \cdot & \cdot & \cdot & \cdot & \cdot \\
-a_{1}-a_{2}-a_{3} & \cdot & \cdot & -a_{n}
\end{array}\right] \underline{x}+\epsilon\left[\begin{array}{c}
0 \\
0 \\
\cdot \\
\cdot \\
0 \\
x_{1}^{3}
\end{array}\right]
$$


with A stable. A RAS is obtained by solving 4.1.5. With $Q=\underline{e}_{1} \underline{e}_{1}{ }^{T}$ where $e_{1}=(1,0,0, \ldots, 0)$. It can be shown (see Lefshetz (43)) that $P$ is $p . d$. if the matrix $C=\left(e_{1}, A^{T} e_{1}, \ldots ., \Delta^{n-1} e_{1}\right)$ has rank $n$.

For this $A, C=I$ and hence $P$ is p.d. (Smith (53) gives a method for the determination of the $p_{i j}$ )

Consider the case for $n=3$ where for A stable the following Routh-Hurwitz conditions must hold (17)

$$
a_{3}>0, a_{3} a_{2}-a_{1}>0, a_{1}>0
$$

For the moment put $\epsilon=1.0$ then the required $P$ is

$$
P=\left[\begin{array}{ccc}
a_{1} d+a_{2} & a_{2} d^{3} & 1 \\
\cdot & \frac{d\left(a_{3}^{3}+a_{1}\right)}{a_{3}^{2}} & d \\
\cdot & 0 & \frac{d}{a_{3}}
\end{array}\right]_{4.2 .26}^{\frac{1}{a_{1}}}, d=\frac{a_{3}^{2}}{\left(a_{3} a_{2}-a_{1}\right)}>0
$$

With $V=\underline{x}^{T} P \underline{x}$ the constraint equation is

$$
\dot{v}=-x_{1} 2\left(1-\frac{1}{a_{1}}\left(x_{1}^{2}+d x_{1} x_{2}+\frac{a}{a_{3}} x_{1} x_{3}\right)\right)=0 \quad 4.2 .27
$$

Of the tangency equations, the equation

$$
\frac{v_{x_{2}}}{v_{x_{3}}}=\frac{\dot{v}_{x_{2}}}{\dot{v}_{x_{3}}}
$$

gives $x_{2}=-a_{3} x_{1}$. The other equation

$$
\frac{v_{x_{1}}}{v_{x_{2}}}=\frac{\dot{v}_{x_{1}}}{\dot{v}_{x_{2}}}
$$


is more complex. However since $\dot{V}$ in 4.2 .27 is nonvanishing on a non-trivial trajectory of the system, the only component of interest is

$$
\left.x_{3}=\frac{a_{3}}{d} \frac{\left(a_{1}\right.}{x_{1}}-x_{1}-d x_{2}\right)
$$

Substituting the latter and the result of 4.2 .28 into 4.2.29 we easily obtain an equation in $x_{1}$ only, reducing to $x_{1}^{4}=a_{1}\left(a_{3} a_{2}-a_{1}\right)$. Thus the valid RS tangency point is. $\mp \underline{\underline{x}}$, where

$$
\begin{aligned}
& \bar{x}_{1}=\left(a_{1}\left(a_{3} a_{2}-a_{1}\right)\right)^{\frac{1}{4}} \\
& \bar{x}_{2}=-a_{3} \bar{x}_{1} \\
& \bar{x}_{3}=\frac{\bar{x}_{1}^{3}}{a_{1} a_{3}}\left(a_{1}+\bar{x}_{1} 2\left(a_{3}-1\right)\right)
\end{aligned}
$$

Also $v_{m}=\left(a_{1} / d\right)^{\frac{1}{2}}$ and since $d(P)=\left(\frac{d a_{1}}{a_{3}^{2}}\right)^{2} \frac{1}{8 a_{1}^{3}}$, the determinant of $P$,

$$
\rho(P)=\pi 2 \sqrt{2} \quad a_{3}^{2}\left(\frac{a_{1}^{5}}{d^{7}}\right)^{\frac{1}{4}}
$$

For any $\epsilon$ an RAS is obtained with becourse to the following theorem which holds when $g(x)=\epsilon g_{k}(x)$; with $g_{k}$ homogeneous of degree $k$.

\section{Theorem 4.1.}

If $V=\underline{T} P_{X}=V_{m}$ is a quadratic boundary for 4.1 .1 which has a tan. pt. at $\underline{x}=\underline{x}^{*}$ for $\epsilon=1.0$, then for any $\epsilon>0,4.1 .1$. has a tangency point $x=x^{*} / \epsilon^{\frac{1}{k-1}}$ which lies on the boundary $v=v_{m} / \epsilon^{\frac{2}{k-1}}$. 
Proof:

$$
\begin{aligned}
& \text { For system } 4.1 .1 \text { with } g=\varepsilon_{I_{k}} \text { we have } \\
& \dot{v}\left(\underline{x} / \varepsilon^{\left(\frac{1}{k-1}\right)}\right)=\frac{1}{\varepsilon^{\left(\frac{2}{k-1}\right)}}\left(-\underline{x}^{T} Q \underline{x}+2 \varepsilon^{\left(\frac{k}{k-1}\right)} \underline{T}^{T} P_{\Phi_{j k}}\left(\underline{x} / \varepsilon^{\frac{1}{k-1}}\right)\right.
\end{aligned}
$$

and since $g_{k}(\underline{x})$ is of degree $k$

$$
\dot{v}\left(x / \varepsilon^{\left(\frac{1}{k-1}\right)}\right)=\frac{1}{\varepsilon^{\left(\frac{2}{k-1}\right)}} \dot{V}(x)
$$

Let $\underline{W}(\underline{x})=\underline{\nabla V}(\underline{x})+\lambda \underline{\nabla} \dot{V}(\underline{x})$, then

$$
\begin{aligned}
& \underline{W}\left(\underline{x} / \varepsilon^{\left(\frac{1}{k-1}\right)}\right)=\frac{1}{\varepsilon^{\left(\frac{1}{k-1}\right)}}\left[P \underline{x}+\lambda\left(-Q \underline{x}+\varepsilon^{\left(\frac{k}{k-1}\right)} P_{B_{B_{k}}}\left(\underline{x} / \varepsilon^{\left(\frac{1}{k-1}\right)}\right)\right.\right. \\
& \left.+\varepsilon J^{T}\left(\tilde{n}_{\mathrm{n}_{\mathrm{k}}}\left(x / \varepsilon^{\left(\frac{1}{k-1}\right)}\right)_{\mathrm{I}}\right)\right]
\end{aligned}
$$

Since $\left.J \mathscr{G}_{k}(x)\right)$, the Jacobjan of $\mathfrak{g}_{\mathrm{l}}$, is of degree $k-1$ we have

$$
\mathbb{W}\left(x / \varepsilon^{\left(\frac{1}{k-1}\right)}\right)=\frac{1}{\varepsilon^{\left(\frac{1}{k-1}\right)^{(x)}}} \quad 4.2 .29 b
$$

Hence if $\underline{W}(\underline{x})=0$ and $\dot{V}(\underline{x})=0$ are satisfied fox $\varepsilon=1$, $\underline{x}=\underline{x}$ and some $\lambda=\lambda^{*}$, they are satisfied for all $\varepsilon>0$.

For the example above only one tangency point exists for $\varepsilon>0$ and thus occurs at $\underline{x}=\bar{\mp} \underline{\underline{x}} / \sqrt{\varepsilon}$. 
82

\section{Example $4,2.5$}

As a final example consider a generalization of system $\$ 1$

$$
\begin{aligned}
\dot{x}_{1} & =-x_{1}+\varepsilon\left(\begin{array}{c}
i_{1} \\
x_{1}
\end{array}\right)\left(\begin{array}{c}
i_{2} \\
x_{2}
\end{array}\right) \cdot\left(\begin{array}{c}
i_{n} \\
x_{n}
\end{array}\right)=-x_{1}+g_{1} \\
\dot{x}_{2} & =-x_{2} \\
& \vdots \\
& : \\
\dot{x}_{n} & =-x_{n}
\end{aligned}
$$

with $i_{1}>1$. Consider a simple quadratic LF

$$
V=\underline{x}^{T} \underline{x}=\sum_{j=1}^{n} x_{j} 2 a_{j}, a_{j}>0
$$

The constraint and tangency equations reduce to

$$
\dot{v}=2\left(-v+a_{1} \epsilon x_{1} g_{1}\right)=0
$$

and the $(n-1)$ equations

$$
x_{j}^{2}=\frac{a_{1} x_{1}^{2}}{\left(1+i_{1}\right)}\left\{\frac{i_{j}}{a_{j}}\right\} \quad j=2, \ldots, n
$$

$4.2 \cdot 31$

Substitution of 4.2 .31 into 4.2 .30 gives an eqn. in $x_{1}$ from which the tangency points follow. Then $V_{m}$ is given a.s.

$$
v_{m}=a_{1}\left[\sum_{j=1}^{n}\left(\frac{a_{j}}{a_{1}}\right)^{i}\right]^{\frac{1}{\left(\sum_{j=1}^{\left.i_{j}-1\right)}\right.} \cdot k}
$$


where

$$
\begin{aligned}
& K=\frac{\left(1+\sum_{j=1}^{n} i_{j}\right)}{\left(1+i_{1}\right)}\left[\frac{\left(1+\sum_{j=1}^{n} i_{j}\right)\left(1+i_{1}\right)^{\left(\frac{1}{2} \sum_{j=2}^{n} i_{j}-1\right)}}{\epsilon \prod_{j=2}^{n}\left(i_{j}\right)^{i_{j} / 2}}\right]^{m} \\
& m=2 /\left(\sum_{j=1}^{n} i_{j}-1\right)
\end{aligned}
$$

and for the volume $\rho(D)=\frac{\pi_{m}^{v_{m}}}{\sqrt{\Gamma^{T}{ }_{j}}}$

The example shows that for some choices of the $i_{j}$ the volume becomes unbounded. For, select

$$
n=2, \quad i_{1}=2, i_{2}=2, \epsilon=2 \text {. }
$$

The system is

$$
\begin{aligned}
& \dot{x}_{1}=-x_{1}+2 x_{1}{ }^{2} x_{2}{ }^{2} \\
& \dot{x}_{2}=-x_{2}
\end{aligned}
$$

and $V=K d_{1}\left(\frac{d_{2}}{d_{1}}\right)^{2 / 3}$ with $\rho=\pi K\left(\frac{d_{2}}{d_{1}}\right)^{1 / 6}$

Put $a_{1}=1.0$ and let $a_{2} \rightarrow \infty$; then $\rho \rightarrow \infty$ but the quadratic boundary is of poor shape approaching the straight line $x_{2}=0$. The actual DOA is $x_{1} 2_{2}<3 / 2$.

The system $S 5$ is useful for test purposes since its DOA is always an open region given by

$$
i_{1}^{i_{1}-1} x_{2}^{i_{2}} \cdot x_{n}^{i_{n}}\left(i_{1}-1\right)+\left(1-\sum_{1}^{n} i_{j}\right)<0 .
$$




\subsection{Numerical Determination of $A$ RAS and an Ontimal Quadratic}

Clearly, finding the optimum RAS for a given class of LF's even as simple as quadratics is difficult analytically. In viev of the work in the previous section a more comprehensive study of second order systems is needed to investigate the relationship between the optimum quadratic boundary, the number of valid tangency points and the components of the constraint contour. Consequentlys a special algorithm, both efficient and accurate, was developed to study the restrictive class of system

$$
\begin{aligned}
& \dot{x}_{1}=f\left(x_{1}, x_{2}\right)=f_{1}+f_{2}+\cdots+f_{n f} \\
& \dot{x}_{2}=g\left(x_{1}, x_{2}\right)=g_{1}+g 2+\cdots+g_{n f}
\end{aligned}
$$

Where $f_{i}, g_{i}$ are homogeneous of degree $i$ in $x_{1}, x_{2}$ and the linear part is assumed stable. The algorithm considers a general LF of the usual form

$$
V\left(x_{1}, x_{2}\right)=v_{2}+v_{3}+\cdot \cdot+V_{I n V} 4.3 .2
$$

where $v_{i}=\sum_{j=1}^{i+1} a_{i j} x_{1}^{i-j+1} x_{2}^{j-1}, v_{2}=\underline{x}^{T} P \underline{x}$ and $P$ solves 4.1 .5 . Consider $V$ and $\dot{V}$ in polar coordinates $(r, \theta)$ then

$$
v(r, \theta)=\sum_{i=2}^{m v} \Psi_{i}(\theta) r^{i}
$$

where

and

$$
\Psi_{i}(\theta)=\sum_{j=1}^{i+1} a_{i j} \cos (\theta)^{i+j-1} \sin (\theta)^{j-1}
$$

$$
\dot{\mathrm{V}}=\mathrm{r}^{2} \sum_{i=2}^{\mathrm{m}} \phi_{\mathrm{i}}(\theta) \frac{i}{\mathrm{r}}-2, \mathrm{~m}=\mathrm{m}_{\mathrm{V}}-\mathrm{n} \mathrm{f}-1
$$

where for 
$i \leqslant m v, \phi_{i}=\sum_{j=1}^{\min (i-1, n f)}\left(\frac{\partial v_{i+1-j}}{\partial x_{1}} \cdot f_{j}+\frac{\left.\partial v_{i+1-j} \cdot s_{j}\right)}{\partial x_{2}}\right.$

$i>m v, \phi_{i}=\sum_{j=i+1-m v}^{\min (i-1, n f)}\left(\frac{\partial v_{i}+1-j}{\partial x_{1}} \cdot f_{j}+\frac{\left.\partial v_{i+1-j} \cdot g_{j}\right)}{\partial x_{2}}\right.$

where for example $f_{j}=f_{j}(\cos \theta, \sin \theta)$ and

$\frac{\partial v_{j}}{\partial x_{1}}=\sum_{j=1}^{i} a_{i j}(i+1-j) \cos (\theta)^{i-j i n}(\theta)^{j-1}$

$\frac{\partial V_{j}}{\partial x_{2}}=\sum_{j=2}^{i+1} a_{i j}(j-1) \cos (\theta)^{j+1-j} \sin (\theta)^{j-2}$

These equations hold by comparing like povers of $r$. Given $\theta$, a point on the contour $\dot{v}=0$ is then found by solving, via 4.3.5, an algebraic equation of degree $m-2$ in $x$ for the root of smallest macmitude, $\bar{r}$ say. At the point $(\bar{r}, \theta), V=V(\bar{r}, \theta)$ and $\dot{V}(\bar{r}, \theta)=0$.

Thus the problem 4.1 .10 reduces to

Where

$$
\begin{array}{cc}
\min _{\theta} V(\bar{r}, \theta) & 4.3 .9 \\
\sum_{i=2}^{m} \phi_{i}(\theta) \bar{r}^{i-2}=0 & 4.3 .10
\end{array}
$$

Henceforth we restrict 4.3 .1 and 4.3 .2 such that $m=m v+n f-1 \leq 6$ so that 4.3 .10 is at most a polynomial of degree 4 in $\bar{r}$ (iTote $\phi_{2}, \psi_{2} \neq 0, \forall \theta$ for $Q$ positive definite) We now describe the main subalgorithms: 
Root Finding Algorithm

Solution of 4.3 .10 for its roots $r_{1}(i=1, m-2)$ is possible by iterative techriques (Froberg (8)) but for $\mathrm{m} \leqslant 6$ ali roots are obtalnable analytically; those of third and fourth degree being solved by Cardan's and Descarte's method respectively (see Froberg (8)).

When searching along the constraint contour $(\dot{V}=0)$ for tangency it is necessary to keep successive solutions of 4.3 .10 on the same component. This is done by finding the root of smallest magnitude, $\vec{x}$, of the same sign as the previous root, $r_{0}$ say. It is also convenient to replace a large or complex root by some upper bound, Rmax say. Thus $\left|r_{j}\right|>\operatorname{Rmax}$ for some $\theta$ indicates that no point on $\dot{V}=0$ lies along the ray $(r, \theta)$ except $r=0$.

Similar considerations apply when solving

$$
\sum_{2}^{m v} \psi_{i} r^{i}=c
$$

for a point on $\mathrm{V}=$ constant say.

A general flow diagram is indicated in Fig. 4.12 where $I V$ is an indicator set to $\circ$ or 1 and $\epsilon$ a small number testing the leading coefficient of 4.3 .10 for zero value. (typically $\epsilon=10^{-6}$ ).

\section{Direct Search Algorithm}

Problem 4.3 .9 can now be regarded as finding the minimum of a scalar function $V(\theta)=V(\bar{r}(\theta), \theta)$. Starting from some initial point $\left(r_{0}, \theta_{0}\right)$ on $\dot{V}=0$, a sequence of values $\theta_{j}$ is determined. Let $v_{j}=v\left(\theta_{j}\right)=v\left(\bar{r}_{j}, \theta_{j}\right)$ 
and s1 be a suitable step length. Then Fig. 4.13 gives the flow diagram which derives 3 values of $\theta, \theta_{1}<\theta_{2}<\theta_{3}$, Which bracket $\theta$, the value giving a relative minimum of $V(\theta), V^{*}$ say. A predicted minimum at $\theta \mathrm{m}$ is found via a weII known quadratic fit (see I.C.I monograph (41)) procedure, which can be repeated on the best three values of $\theta_{m}, \theta_{1}, \theta_{2}, \theta_{3}$ that bracket the minimun (not now equaliy spaced). Convergence is obtained when either a) $\left|\theta_{m}-\vec{\theta}_{m}\right|<10^{-t}$, where $\theta_{m}$ and $\bar{\theta}_{m}$ are sucessive predicted minima of $\theta^{*}$ or $\left.\mathrm{b}\right)$ the number of repetitions exceeds some integer, I say; whichever comes first (typically $I=5$ and $t=3$ ). Finally, an upperbound is placed on the step length s1 of Smax which if exceeded restarts the initial search for the bracketing values. (Smax $=.15$ rad. is suitable).

\section{Algorithm for RAS Determination}

The main steps in finding a RAS for the IF 4.3 .2 are as follows:

(a) with $s=\pi / N \quad(N=10$ say $)$ let $\theta_{j}=j s, j=0,1, \ldots, N$. Determine $\min _{j} V\left(\theta_{j}\right)$ $=\min _{j} V\left(\vec{r}_{j}, \theta_{j}\right)$ and let $\theta^{*}$ be the min$\operatorname{imizing} \theta_{j}$ If all $\left|r_{j}\right| \geq R \max$ increase $N$ and repeat (a) otherwise (b).

(b) with $\theta_{0}=\theta^{*}\left(\dot{V}\left(\bar{r}^{*}, \theta^{*}\right)=0.0\right)$ find the nearest $\theta_{\mathrm{m}}$ via the direct'search algorithm. Then $\left(\vec{r}_{m}, \theta_{m}\right)$ is a tangency point and $V=V\left(\bar{r}_{m}, \theta_{m}\right)=V_{m}$ a possible RAS boundary. 
Choose a conservative boundary (since tan. pt. not exact) of $v=v\left(r, \theta_{m}\right)=\bar{v}_{m}$ with $r=\bar{r}_{m}(1-\gamma)$ $(\gamma=.01$ in practice). Test tan. pt. via step (c).

(c) Select $s_{1}=2 \pi / N 1$. For $\theta_{j}=\theta_{m}+j s_{1}, j=1$, -, $\mathrm{N} 1$, find $\bar{r}_{j}$ the valia root of $\sum_{i=2} \psi_{i}\left(\theta_{j}\right) r^{i}=\bar{v}_{m}$. Calculate $\dot{V}_{j}=\dot{v}\left(\bar{r}_{j}, \theta_{j}\right)$. If for some $j, \dot{v}_{j-1}<0$ but $\dot{v}_{j}>0.0$ tangency is invalid; put $\theta^{*}=\theta_{j}$ and repeat $(b)$. Otherwise (d).

(d) Find $\rho(v)$, plot contours of $v=v_{m}$ or $\dot{V}=0$ if required.

Step (a) insures a valid initial point on $\dot{V}=0$ near the origin and (c) insures a valid tangency (typicaliy, $\mathrm{N1}=50$ ). Also the search in (c) is reduced if the smallest root of opposite sign to $\bar{r}_{j}$ is found, then $s_{1}=\pi / N 1$ with $N_{1}=25$ say. The algorithm, called REGION, was programmed in FORTRAN IV and determines RAS's of quite general LF's.

\section{Optimal Quadratic Algorithm}

Algorithm REGION, in conjuction with Powell's conjugate gradient algorithm (33), was used to obtain optimal quadratics for system 4.3.1. The constraint on $Q(Q$ p.d.) was avoided by maximizing $\rho$ over an upper triangular matrix $L$ s.t. $Q=I^{\top} I+\epsilon I, \epsilon>0$. Thus

$$
L=\left[\begin{array}{ll}
1 & t_{1} \\
0 & t_{2}
\end{array}\right], \quad Q=\left[\begin{array}{lr}
1 & t_{1} \\
t_{1} & t_{1}^{2}+t_{2}^{2}
\end{array}\right] \geqslant 0
$$


Considered as a function of $t_{1}$ and $t_{2}, \rho\left(t_{1}, t_{2}\right)$ was found by REGION for any $\left(t_{1}, t_{2}\right)$ which was a function value input to Povell's routine. Fig. 4.14 shows the interaction of the various routines.

\subsection{Numerical Results}

In view of Example 4.2.1. and the results that follow we make the following definition w.r.t. measure $\rho(v)$.

\section{Def 4.4}

An asymptotically stable system has property $A$ w.r.t. an optimal quadratic $(O Q)$ and its (RS) constraint contour if a) at least two valid (RS) tangency points exist or b) only one (RS) valid tangency point exists, there being only one tangency point for any general quadratic. (Note, an $O Q$ having two RS tangency points but only one RS valid tangency point would not satisfy property $A)$.

System $S 1$, satisfying (a), and $S 3$, satisfying (b) with $\alpha=\beta=\epsilon=1.0$, were shown to have this property. In what follows the property will hold to a certain accuracy in that if $\underline{x}_{1}$ is valid tangency point and $x_{2}$ any other then the property holds if

$$
\frac{\left|V\left(\underline{x}_{1}\right)-V\left(\underline{x}_{2}\right)\right|}{V\left(x_{1}\right)}<\epsilon \quad\left(\epsilon=10^{-3} \text { say }\right)
$$

In order to generate systems with say $k$ components of $\mathrm{E}_{\mathrm{V}}$, and thus $\mathrm{k}$ possible tangency points, consider the system 


$$
\begin{aligned}
& \dot{x}_{1}=x_{2} \\
& \dot{x}_{2}=-\alpha x_{1}-\beta x_{2}+f_{k}\left(x_{1}, x_{2}\right) \quad \text { 4.4.1. }
\end{aligned}
$$

$\left(f_{k}\right.$ homog. deg. $\left.k\right)$. Then $E_{V}$ consists of points $(r, \theta)$ where

( $\mathrm{p}_{i j}$, elements of $P$ )

$$
{ }_{r}^{(k-1)}=\frac{\underline{y}^{\top} Q_{L}}{2\left(p_{21} y_{1}+p_{22} y_{2}\right) f_{k}}=\frac{G(\theta)}{F\left(y_{1}, y_{2}\right)}
$$

with $y_{1}=\underset{k+1}{\cos \theta,} y_{2}=\sin \theta$. Clearly, $w$ may be factored as $F=b \prod_{j=1}^{K+1}\left(y_{2}-a_{j} y_{1}\right) ;$ and by suitable choice of $a_{j}$ (real or conf. complex) the lines $y_{2}=$ aj $y_{1}$ partition $\mathrm{E}^{2}$ into regions in which either $1,2, \ldots, k$ or $k+1$ (RS) components of $\mathrm{E}_{\mathrm{V}}$ lie for $\mathrm{k}$ odd (even).

System 56 (Davies (44))

$$
\begin{aligned}
& \dot{x}_{1}=6 x_{2}-2 x_{2}^{2} \\
& \dot{x}_{2}=10 x_{1}-x_{2}+4 x_{1}^{2}+2 x_{1} x_{2}+4 x_{2}^{2}
\end{aligned}
$$

s6

The singular points are $P_{1}(0,0)$ and $P_{2}(0,2.5)$. The DOA is a limit cycle region, $\left(x_{1}-.5\right)^{2}+x_{2}^{2}<1.0$

For any quadratic the system has one component of $E_{V}$ but Fig. 4.4. shows an initial quadratic with one valio tangency and the unique $O Q$ With two. The $O Q$ gives a poor RAS.

\section{System S2 (Van der Pol)}

Consider Example 4.2.2. With $\epsilon=1.0$. Fig. 4.5(a) shows the $O Q$ boundary in relation to the two RS components of $E_{V}$. The $O Q$ is unique with two valid tangency points. 
Fig. 4.5(b) shows the variation of the OQ with $\epsilon$. Its RAS increases both in area and elongation and reflects the change in the DOA. Property $A$ held in all cases.

\section{System S7}

$$
\begin{aligned}
& \dot{x}_{1}=x_{2} \\
& \dot{x}_{2}=-x_{1}-4 x_{2}+\frac{1}{4}\left(x_{2}-.5 x_{1}\right)\left(x_{2}-2 x_{1}\right)\left(x_{2}+2 x_{1}\right) \\
& \left(x_{2}+x_{1}\right)
\end{aligned}
$$

The singular points are $P_{1}(0,0), P_{2}(\sqrt[3]{2}, 0)$

the latter beingasaddle point .

For a general quadratic $\mathrm{E}_{\mathrm{v}}$ may have 5 components and the valid tangency alternates between two of these near the optimum. The $0 Q^{\prime}$ 's obtained via Powell for different Initial $\left(t_{1}, t_{2}\right)$, varied considerably. Three supposedy $O Q^{\prime}$ 's are shown in Fig. 4.6. Each has two valid tangency points so we might suspect a non-unique optimum, as for system S1. However, consider Fig. 4.7 which shows the contours of $\rho(\rho=$ av radius $)$ in the $t_{1}-t_{2} p$ lane. There exists an equal tangency curve - shown $A B$ - such that any point lying on it produces a quadratic with two valid tangency points. Infact the three quadratics $V_{1}$, $V_{2}$ and $V_{3}$ in Fig. 4.6 correspond to the points $P_{1}, P_{2}, P_{3}$ on this curve - tangerey cccurring on component $S$ for points near and above $A B$ and on $T$ for points below $A B$.

The reason for the bad convergence of Powell is the 'sharp corners' of the $\rho$ - contours on $A B$. For then the assumption that $\rho$ may be approximated by a quadratic, 
on which Powell is based, breaks down with a consequent loss in search direction.

In part, this expiains some results of Bream (45) who showed the inferiority of Powell compared to the simplex method of Nelder and Mead (46) when optimizing $p$ for Zubov functions obtained from series 3.2.3. For higher degree LF's this lack of convergence would be even more prominent.

The Simplex method, not relying on gradients or quadratic fits, still retains a flexible search direction even when $A B$ is reached.

System $S 7$ also exhibits a problem when using any optimization routine. Consider Fig. 4.7. With a poor initial guess, $C$ say. Searching along the gradient or coordinate drections in turn, we arrive at $E$ or $C_{1}$ on $A B$. Further improvement is only made by moving along $A B$ in an oscillatory fashion. As the valid tangency alternates between component $S$ and $T$, repeated use of step (c) is needed in algorithm REGION. This increases computer time.

In contrast to 57 , Fig. 4.8 shows the $\rho$-contours for the Van der Pol system. Again, the equal tangerncy curve is present - $A B$ - but Powell's method has little difficulty In reaching the optimum since the contours are quite smooth and $A B$ is almost parallel to the $t_{2}$ axis.

\section{System 88}

A system giving 3 components for $\mathrm{E}_{\mathrm{V}}$ is 


$$
\begin{aligned}
& \dot{x}_{1}=-2 x_{1}+x_{1} x_{2} \\
& \dot{x}_{2}=-x_{2}+x_{1} x_{2}
\end{aligned}
$$

with singular points $P_{1}(0,0)$ and $P_{2}(1,2)$. Fig. 4.9 shows three quadratic boundaries with their associated constraint contours, while Fig. 4.10, the $\rho$-contours $(\rho=$ area). In the latter $V_{1}$ corresponds to the initial point $I(1,1)$; $V_{2}$ to the point $J(\cdot 206,1 \cdot 0)$, the point reached after searching along $t_{2}=1.0$; and $v_{3}$ to the point $0(\cdot 233, \cdot 449)$, the optimal point. The fact that $I$ and $J$ lie on different sides of $A B$ is illustrated in Fig. 4.10 where $V_{1}$ and $V_{2}$ have valid tangency on different components. The point 0 lies on $A B$ and 'property $A$ ' holds. (Note, there are quadratics which have valid tangency with the third component, but these have small area).

\section{System S9}

Consider a more general system

$$
\begin{aligned}
\dot{x}_{1} & =x_{2} \\
\dot{x}_{2} & =-2 x_{1}-3 x_{2}+\frac{x_{2}^{2}}{4}+\frac{x_{1}^{2} x_{2}}{4} \\
& +x_{2}^{2}\left(x_{1}^{2}+x_{2}^{2}\right) / 4 \\
& +\left(x_{2}-x_{1} / 2\right)^{2}\left(x_{2}-4 x_{1}\right)\left(x_{2}+.5 x_{1}\right)
\end{aligned}
$$

For a general quadratic IF there exists 5 components of the constraint contour of which only two are of interest near the optimum. Fig. 4.11 shows an initial quadratic, $V_{i}$, with one valid tangency point and the optimum, $v_{0}$, with two. 


\section{Comment}

Many other second order examples have shown sinilar results. Certainly, the examples shown here have in dicated that property $A$ is quite general and that it holds forpchosen as the average radius or the area.

\subsection{Optimal Quadratics for a Restricted Class of High Order} Systems

The optimal quadratic algorithm previousiy outlined can be generalized to the class of systems

$$
\dot{x}=A x+B_{2}+B_{3}+\cdots+g_{n f} \quad 4.5 .1
$$

with $n_{f} \leq 5$, by using the general polar coordinate system in $\left(r, \theta_{1}, \theta_{2}, \quad, \theta_{n-1}\right)$ derined as

$$
\begin{aligned}
& x_{1}=r c_{1} c_{2} \cdot \cdot c_{n-1} \\
& x_{2}=r c_{1} c_{2} \cdot c_{n-2} s_{n-1} \\
& \cdot \\
& x_{1}=r c_{1} c_{2} \cdot \cdots c_{n-1} s_{n-1-1} \\
& \cdot \\
& x_{n}=r s_{1}
\end{aligned}
$$

where $c_{i}=\cos \left(\theta_{i}\right), s_{i}=\sin \left(\theta_{i}\right)$ and $r=\|x\|$. Let $y_{i}=x_{i} / x, i=1$, , n. Then given $\theta_{i}(i=1, \cdot, n-1), I$ is determined, and a point $x$ on the RAS boundary, $\underline{x}^{T} P_{x}=V_{m}$, or the constraint surfa ce, $\dot{V}=0$, is found by calculating 


$$
r=\left(V_{m} / y^{i} P y\right)^{\frac{1}{2}} \quad 4.5 .3
$$

or by solving the polynominal in $r$

$$
\sum_{i=1}^{n_{f}} \phi_{i}(y) x^{i-1}=0 \quad 4.5 .4
$$

where $\phi_{1}=-\underline{Y}^{\mathrm{T}} \mathrm{Q}$ and $\phi_{i}=2 \mathrm{Y}^{\mathrm{T}} \mathrm{P} \mathrm{g}_{i}(\mathrm{y}), i>1$.

The root of smallest magnitude with a given sign of 4.5.4, $\overline{7}$ say, is found by using the ROOTV algorithm and forms the basis of a new algorithm for RAS determination, called DNREG.

For RAS and tangency point determination the problem of minimizing $V$ on the constraint contour is replaced by

$$
\min _{\mathbb{Z}} V(\bar{r}, \mathbb{Z})
$$$$
4.5 .5
$$

With $\bar{r}$ the required root of 4.5.4. The minimization is over the $n-1$ variables of $\theta_{1}$ and for this Powell's $)_{\text {method }}^{(33)}$ was used, replacing the direct search method mentioned previously. The $O Q$ was determined by maximizing $\rho$ by the Simplex Hethod of Nelder and head (46) over the $m$ - dimensional space of the elements of the upper triangular matrix $I$, with $Q=L^{T} L+\in I$ and $m=n(n+1) / 2-1$.

The algorithm was especially developed to study third order systems but is easily extended to $n>3$. In this case the steps for DNREG are those of REGION except that (a) and (c) are now 2 - dimensional searches:
a) Let $s_{1}=\pi / 2 N_{1}$
$(\mathrm{N1}=5$ say $)$
$s_{2}=2 \pi / N_{2}$
$(\mathrm{N} 2=15 \mathrm{say})$ 
With $y=y\left(\theta_{1}, \theta_{2}\right)$, let

$$
\begin{aligned}
v_{i, j} & =y\left(i s_{1}, j s_{2}\right) \\
i & =0,1, \cdot N 1 \\
j & =0,1, \cdot, N 2
\end{aligned}
$$

and determine $\min _{i, j} V\left(\underline{y}_{i, j}\right)=V\left(\bar{r}_{i, j}, Z_{1, j}\right)$ with $\mathbb{x}^{*}$ the minimizing $z_{i, j}$. If all $\left|\vec{r}_{i, j}\right| \geq R \max$ increase $N 1$ and $N 2$ and repeat (a) until some $\left|\vec{r}_{i, j}\right|<\operatorname{Rmax}$. Otherwise step (b).

(c) Tangency is valid if $\max _{\underline{x}} \dot{V}<0$ on $V(\underline{x})=V_{m}$. Let $Y:\left(y_{1}, y_{2}, \cdots, y_{n}\right)$ be a set of unit vectors, determined as in 4.5 .6 but with a finer mesh (N1 $=15$, N2 $=50$ say with $N=16 \times 51)$. Determine

$$
\dot{V}_{M}=\max _{j}\left[\dot{V}\left[\bar{\Psi}\left(\frac{v_{m}}{{\underline{V_{j}}}_{\mathrm{P}_{\mathbf{j}}}}\right)^{\frac{1}{2}} \underline{Y}_{j}\right]\right]
$$

If $\dot{V}_{M}<0$ go to (d) otherwise let $\mathbb{Z}^{*}$ be the maximizing $Z_{j}$ and repeat step (b).

The unit vectors were stored through the Simplex maximization, and also, step (a) was only used for the inftial quadratic, the initial y for the Powell routine being that obtained from the tangency point of the previous quadratic.

The flow diagram of Fig. 4.15 shows how the complete OQ routine was divided into subroutines for FORTRAN IV Programining.

An Initial $L$ determines an initial point of the simplex (a set ofm+1points in $m$-space) written $p_{1}=\left(t_{1}, t_{2}\right.$, .,$\left.t_{\mathrm{m}}\right)$ where 


$$
L=\left[\begin{array}{lll}
t_{1} & t_{2} & t_{4} \\
0 & t_{3} & t_{5} \\
0 & 0 & 1.0
\end{array}\right] \quad\left(t_{6}=1.0, \mathrm{~m}=5\right)
$$

The remaining $m$ points of the simplex were

$$
\begin{gathered}
p_{j+1}=\left(t_{1}, t_{2}, \cdots, t_{j-1}, t_{j}+h, t_{j+1}, \ldots t_{m}\right) \\
j=1, \ldots, m
\end{gathered}
$$

with $h$ a parameter.

In the following examples only 3 iterations ( 30 function evaluations of $\vec{r}$ ) of Powelis method were needed for step (b).

\subsection{Numerical Results}

\section{System S10}

The thiralorder

$$
\begin{aligned}
& \dot{x}_{1}=x_{2} \\
& \dot{x}_{2}=x_{3} \\
& \dot{x}_{3}=-x_{1}\left(1+x_{1}{ }^{2}\right)-2 x_{2}-x_{3}\left(1-x_{3}\right)^{2}
\end{aligned}
$$

For a general quadratic, $\underline{x}^{\top} P_{\underline{X}}=V$, there appears to be two RS tangency points and two RS components of $\mathrm{EV}$, which lie in $\mathrm{E}^{3}$ for which

$$
\left(p_{31} x_{1}+p_{32} x_{2}+p_{33} x_{3}\right)\left(x_{3}-x_{1}\right)>0 \text {. }
$$

With $h=\cdot 3$ and $N=616$ the initial quadratic boundary, with $Q=I$, was

$$
2 \cdot 5 x_{1}^{2}+5 x_{1} x_{2}+x_{1} x_{3}+3 x_{1} x_{3}+5 x_{2}^{2}+2 x_{3}^{2}=.4675
$$

with $\rho=32799$ (Vol!) and valid tangeney 


$$
\mp(\cdot 005,-.004, \cdot 523)
$$

After 61 evaluations of $\rho$ the best boundary was $\underline{x}^{\top} \bar{x} x=.847$ with $\rho=.842$ and tangency $F(.7145,-345,-.367)$, with

$$
\widetilde{\mathbf{P}}=\left[\begin{array}{ccc}
2.144 & 2.232 & .47 \\
. & 4.56 & 1.14 \\
. & . & 2.06
\end{array}\right] \quad 4.6 .1
$$

The other tan. pt. $\mp(-.062,-.091, .682)$ gave a boundary close to this of $\mathrm{V}=.856$.

In general it was found difficult to obtain the $O Q$ accurately due to the high dimension of the problem and the approximations involved. However, the example shows property A is present. Firstly, Fig. 4.16 shows sections of the initial and best quadratics in relation to their constraint contours and DOA's. The section through $x_{3}=.3677$ shows the extra point of contact of $V=V_{m}$ with $E_{V}$ for the $O Q$. Secondly, Fig. 4.17 shows how valid tangency alternates between the two possible tangency points as the maximization of $\rho$ progresses, and indicates an equal tangency surface. The RAS is a good estimate of the DOA.

System Si1 (Davidson (47))

A system possessing a limit cycle is the following:

$$
\begin{aligned}
& \dot{x}_{1}=x_{2} \\
& \dot{x}_{2}=x_{3} \\
& \dot{x}_{3}=-.915 x_{1}+x_{2}\left(1-.915 x_{1}{ }^{2}\right)-x_{3}
\end{aligned}
$$


Again there are two possible RS tangency points for a general quadratic, and two RS components of $E_{\mathrm{V}}$. With $\mathrm{h}=.1, \mathrm{~N}=616$ and an initial $\mathrm{P}$ of $\left.\mathrm{P}=(\mathrm{SS})^{*}\right)^{-1}$ (Eqn. 2.2.2) the RAS boundary was:

$$
\begin{array}{r}
24 \cdot 2 x_{1}^{2}-26 \cdot 4 x_{1} x_{2}+26 \cdot 4 x_{1} x_{3}+25 \cdot 8 x_{2}^{2}-27 \cdot 6 x_{2} x_{3} \\
+27 x_{3}^{2}=2 \cdot 07
\end{array}
$$

with $\rho=.105$ and tan. pt. $\mp(\cdot 249, \cdot 171,-\cdot 223)$. After 90 evaluations of $\rho$ the best $0 \%$ boundary was $x^{T_{P x}}=4.4856$ with $\rho=.767$ and

$$
\mathrm{p}=\left[\begin{array}{ccc}
18.1 & -15.86 & 1.88 \\
\cdot & 31.94 & -16.59 \\
\cdot & \cdot & 17.19
\end{array}\right] \quad \begin{aligned}
& \tan . \mathrm{pt} . \\
& \mp(.376, .271,-.241)
\end{aligned}
$$

The other tan. $p t$. gives the boundary $\underline{x}^{T} P x=4: 534$ which is indistinguishable, graphically, from the OQ. Fig. 4.18 shows sections through the injtial and best boundaries and constraint contours. Those through $\mathrm{x}_{3}=.158$ and $x_{3}=.241$, parallel to the $x_{1}-x_{2}$ plane, show the closeness of the $O Q$ boundary to its two components of $\mathrm{E}_{\mathrm{V}}$. In Fig. 4.19 the oscillatory effect of the valid tangency is shown again.

\section{System $\mathbf{S 1 2}$}

A system giving one RS tan. pt. is

$$
\begin{aligned}
& \dot{x}_{1}=x_{2} \\
& \dot{x}_{2}=x_{3} \\
& \dot{x}_{3}=-x_{1}-2 x_{2}-x_{3}+x_{1}{ }^{3}
\end{aligned}
$$


Which is of the form S4. Using $P$ in 4.2 .26 gives the RAS boundary

$$
3 x_{1}^{2}+4 x_{1} x_{2}+2 x_{1} x_{3}+2 x_{2}^{2}+2 x_{2} x_{3}+2 x_{3}^{2}=2.0
$$

vith $\rho=8.36$ \& tar. pt. $\div(1,-1,1)$. With $h=1$, $N=616$ and $Q=I$, initially, the best quadratic gave $\rho=$ 17.5 after 100 p-evaluations. Due to the single tangency, convergence to the optimum was rapid. The best boundary was

$$
\begin{array}{r}
89 \cdot 4 x_{1}^{2}+96 \cdot 9 x_{1} x_{2}+63 x_{1} x_{3}+89 x_{2}^{2}+48 \cdot 9 x_{1} x_{3}+52 x_{3}^{2} \\
=85 \cdot 45
\end{array}
$$

with $\tan$. pt. $\mp(1.162,-.437, .0953)$

\section{Comments}

Computation times for the 3 examples were 1000,4000 and 1800 secs. respectively (ICL1905), an average function ( $p$ ) evaluation being 16,67 and 18 secs respectively.

Comparison of computation times for second and third order system depends upon the degree and complexity of the system considered and on the values of $N 1$ and $N$ in step (c) of the algorithms. A two fold increase in N1 (with increase in accuracy) may often double computing time. For the second order case with $N 1=30$ an average evaluation took 1 sec, whereas 5 sec. for $N 1=50$.

Generally a compromise must be made between computing time and accuracy of the valid tangency point. For high order systems the validification of the latter via step (c) seems a big arawback, especially so near the optimum due to the oscillatory effect of the valid tangency and repetition of this step. 


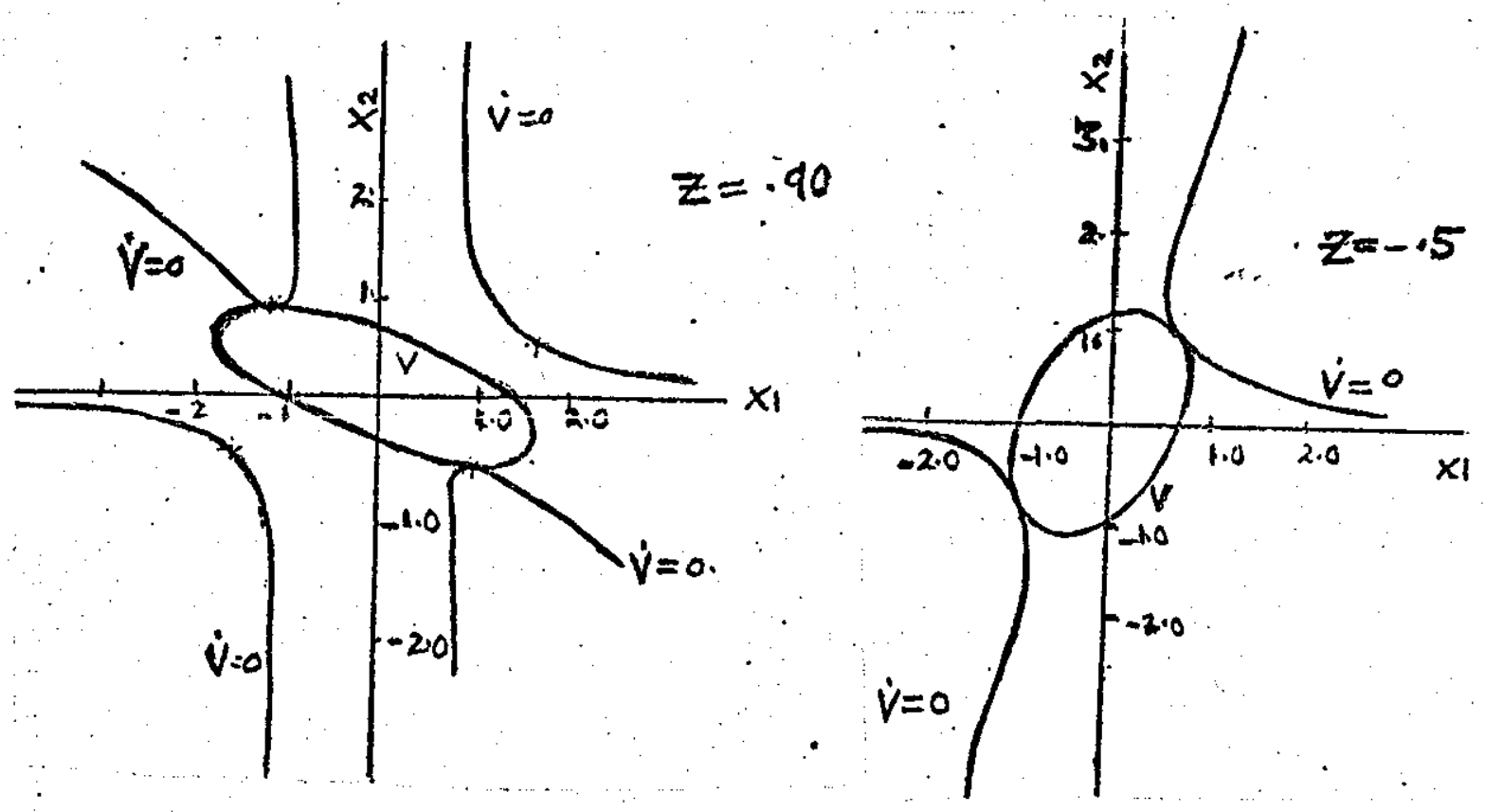

$V=0.625 x_{1}^{2}+2.0 x_{1} x_{2}+2 x_{2}^{2}=288, V=4 x_{1}^{2}-2.0 x_{1} x_{2}+x_{2}^{2}=1.24$

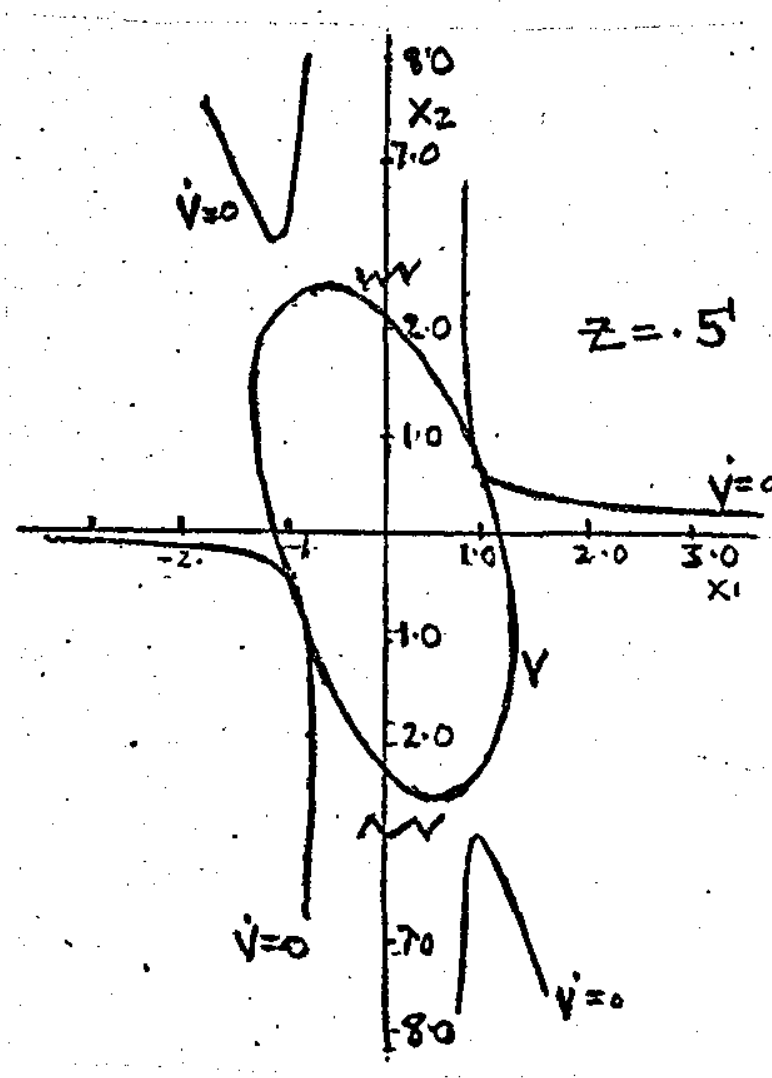

$Y=4.0 x_{1}^{2}+2 x_{1} x_{2}+x_{2}^{2}=4.5$

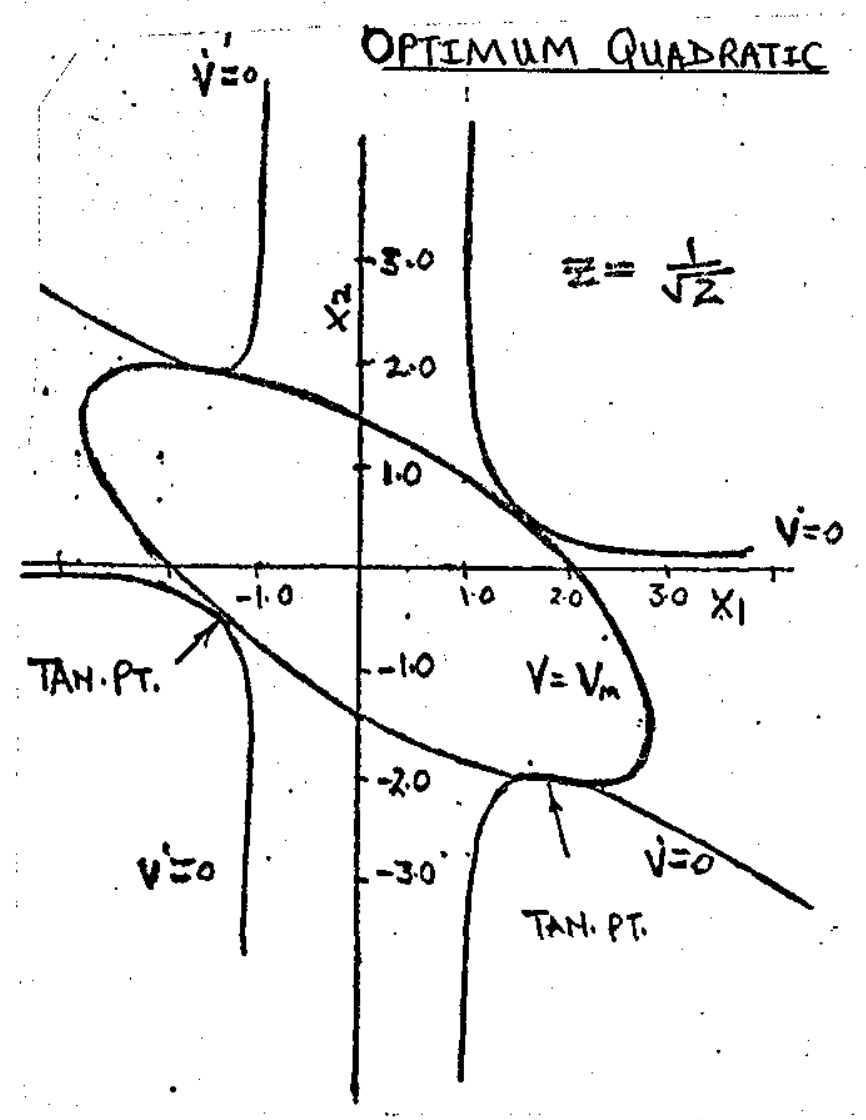

$V=x_{1}^{2}+2 x_{1} x_{2}+2 x_{2}^{2}=4 \cdot 0$

RAS BOUNDARIES AND CONSTENINT CONTOURS (ZUBOV EXPLE.) 

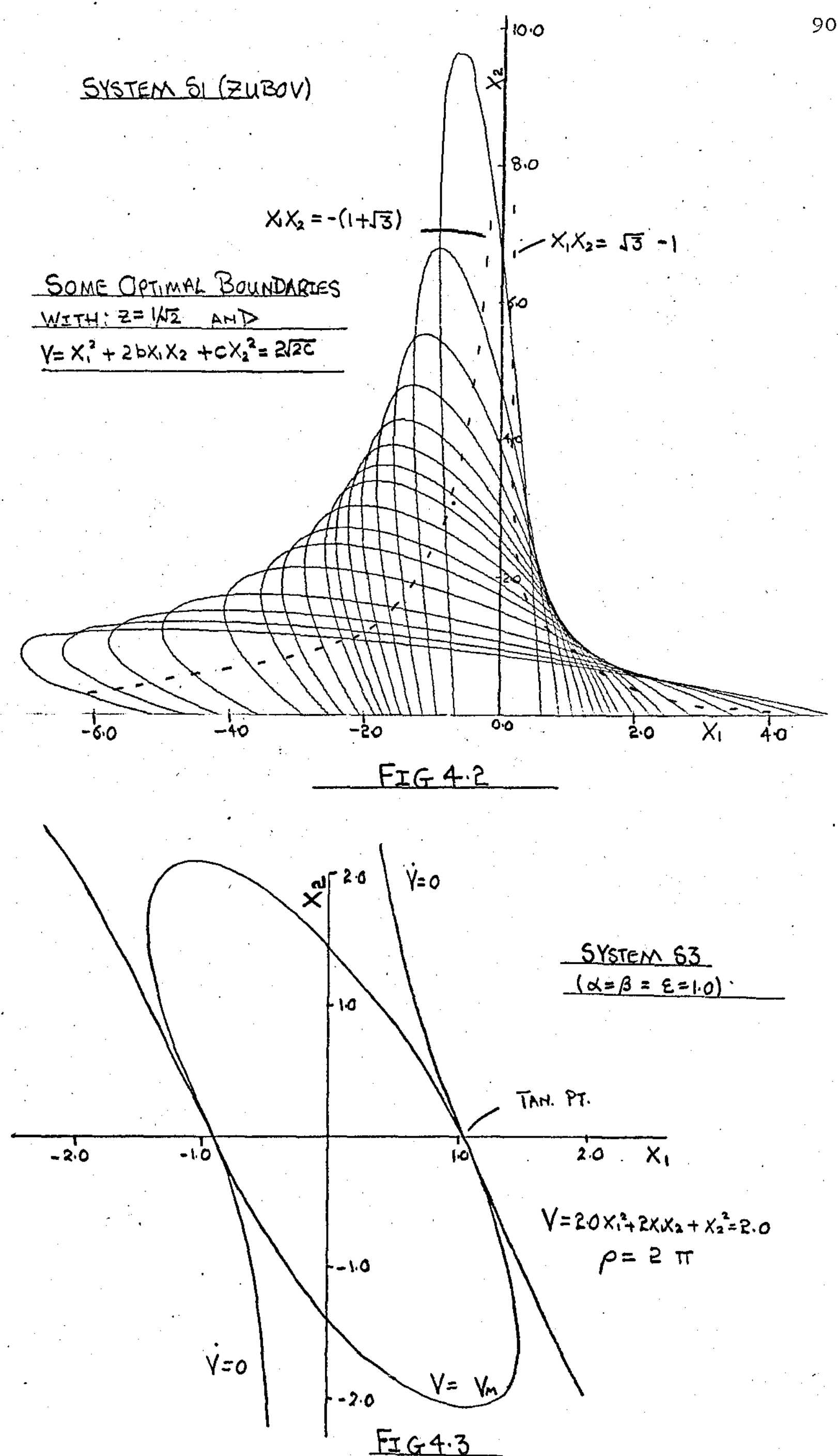
91

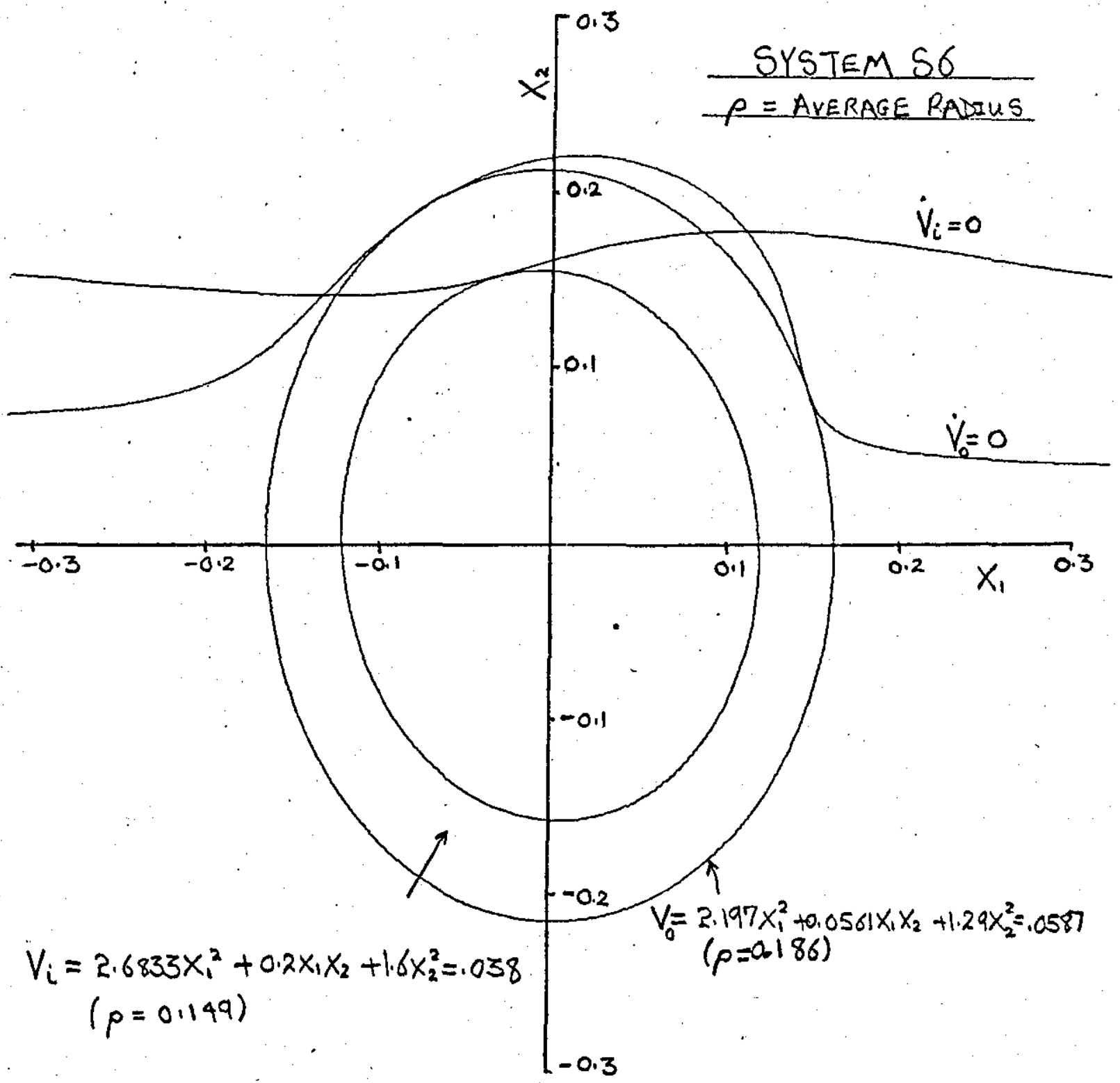

FIG. 4.4

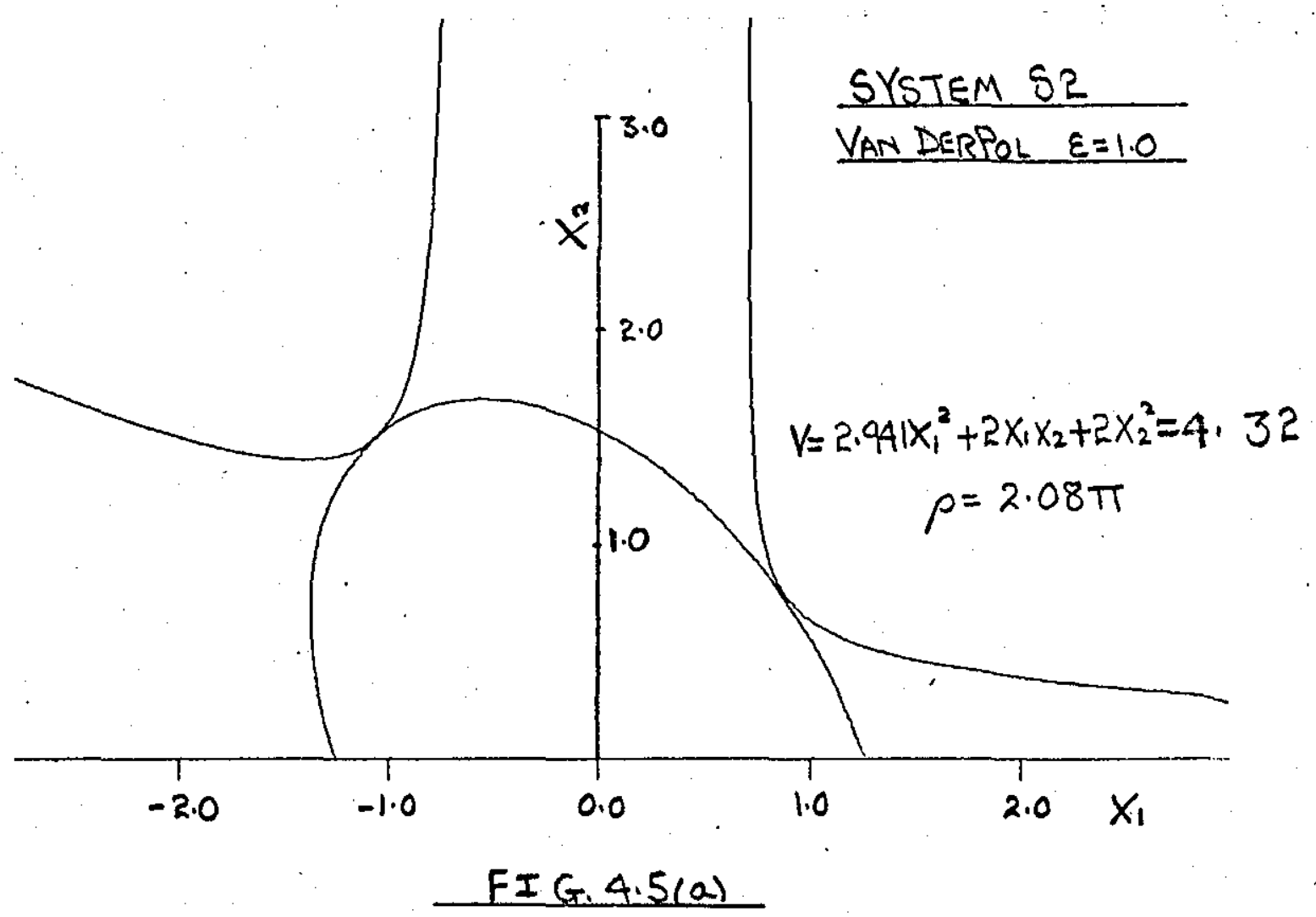



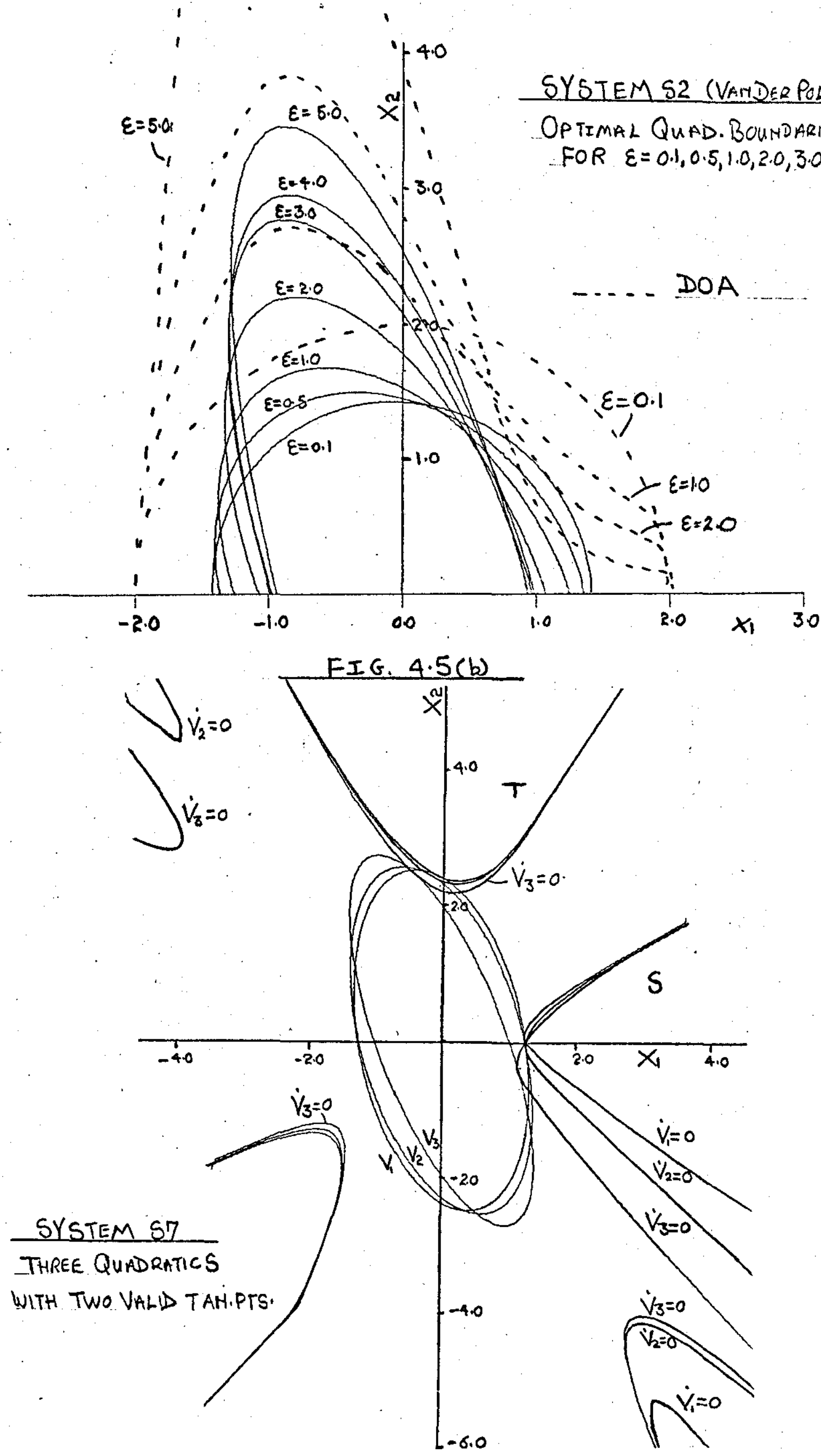

FIG. 4.6 

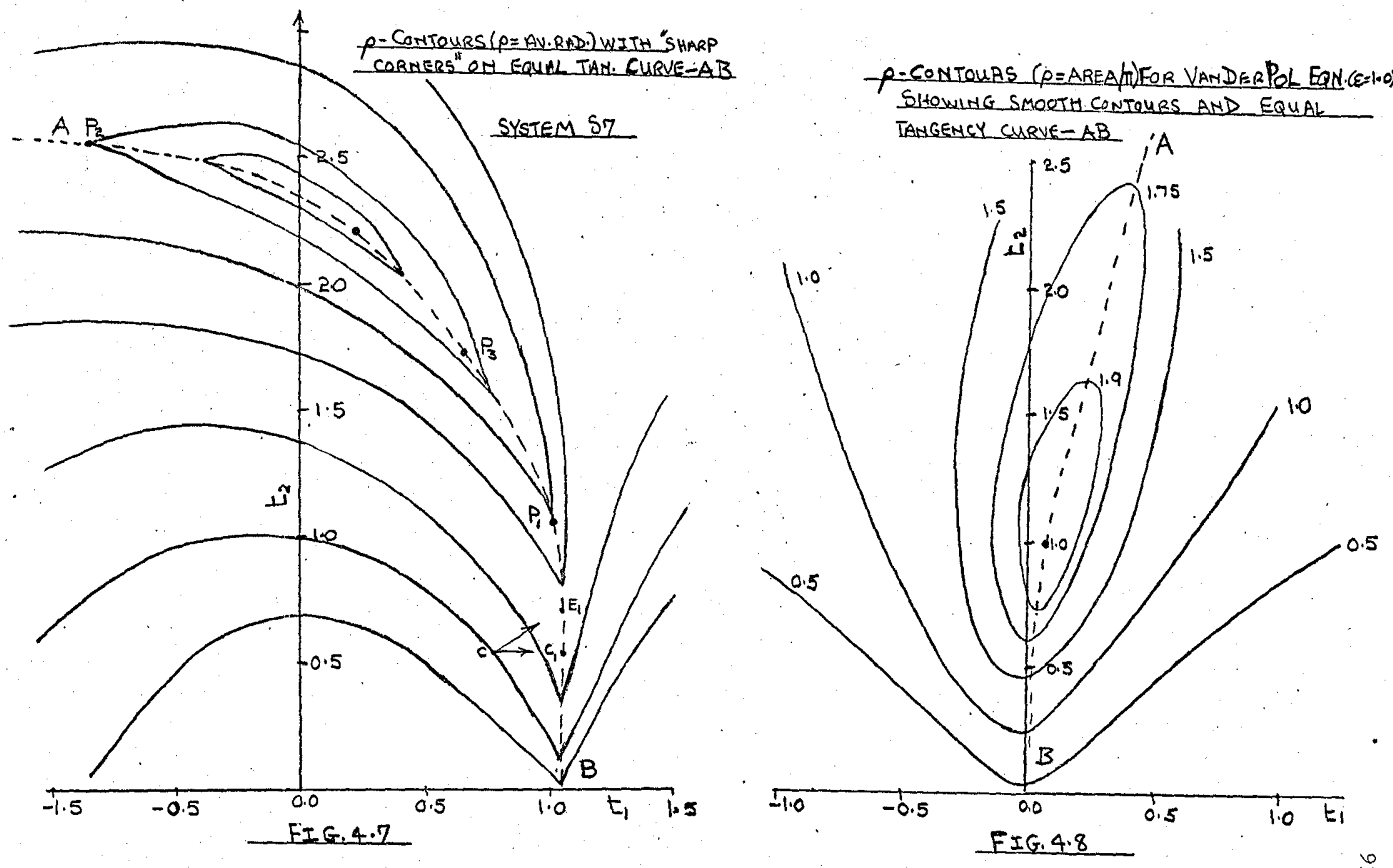


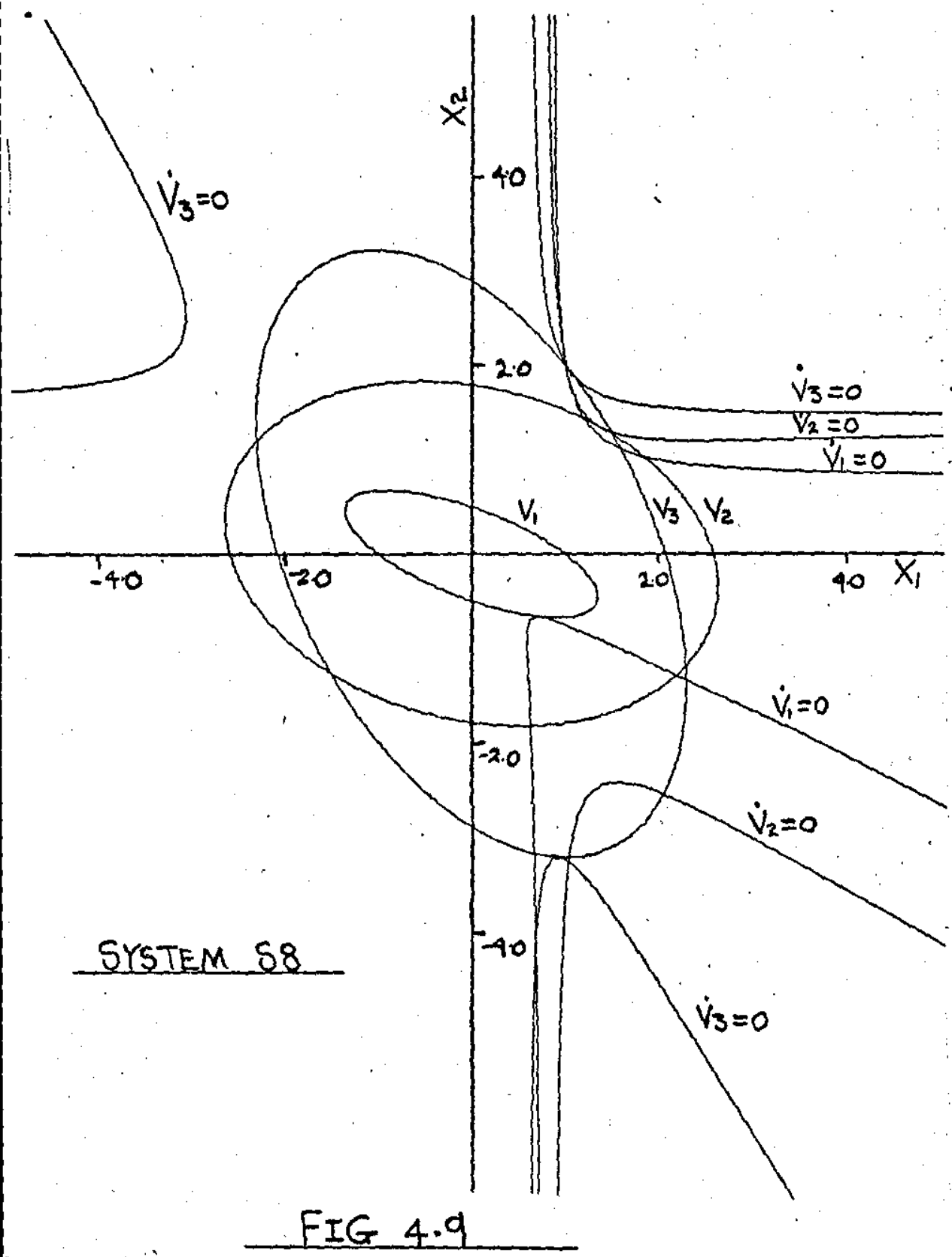




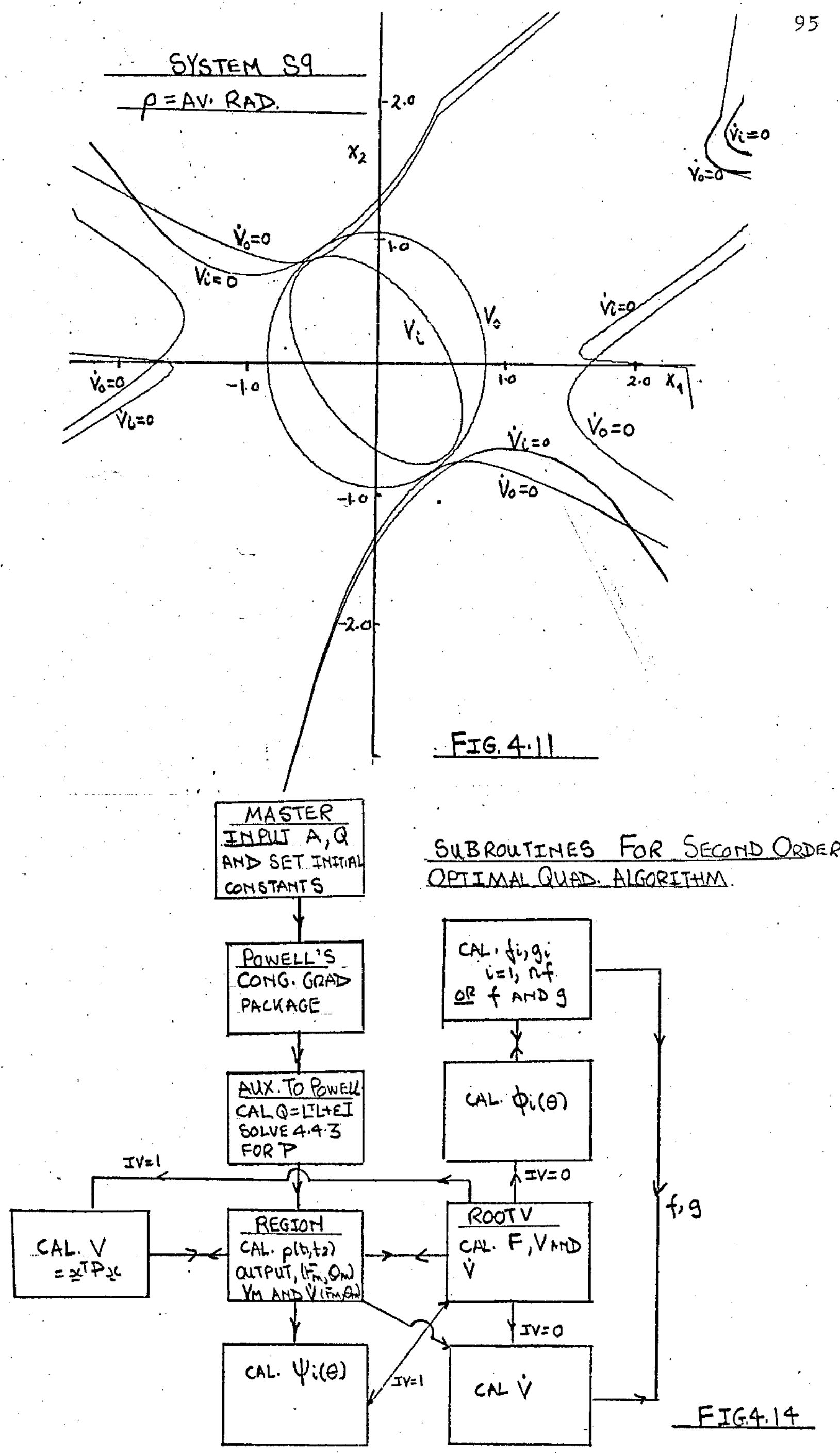




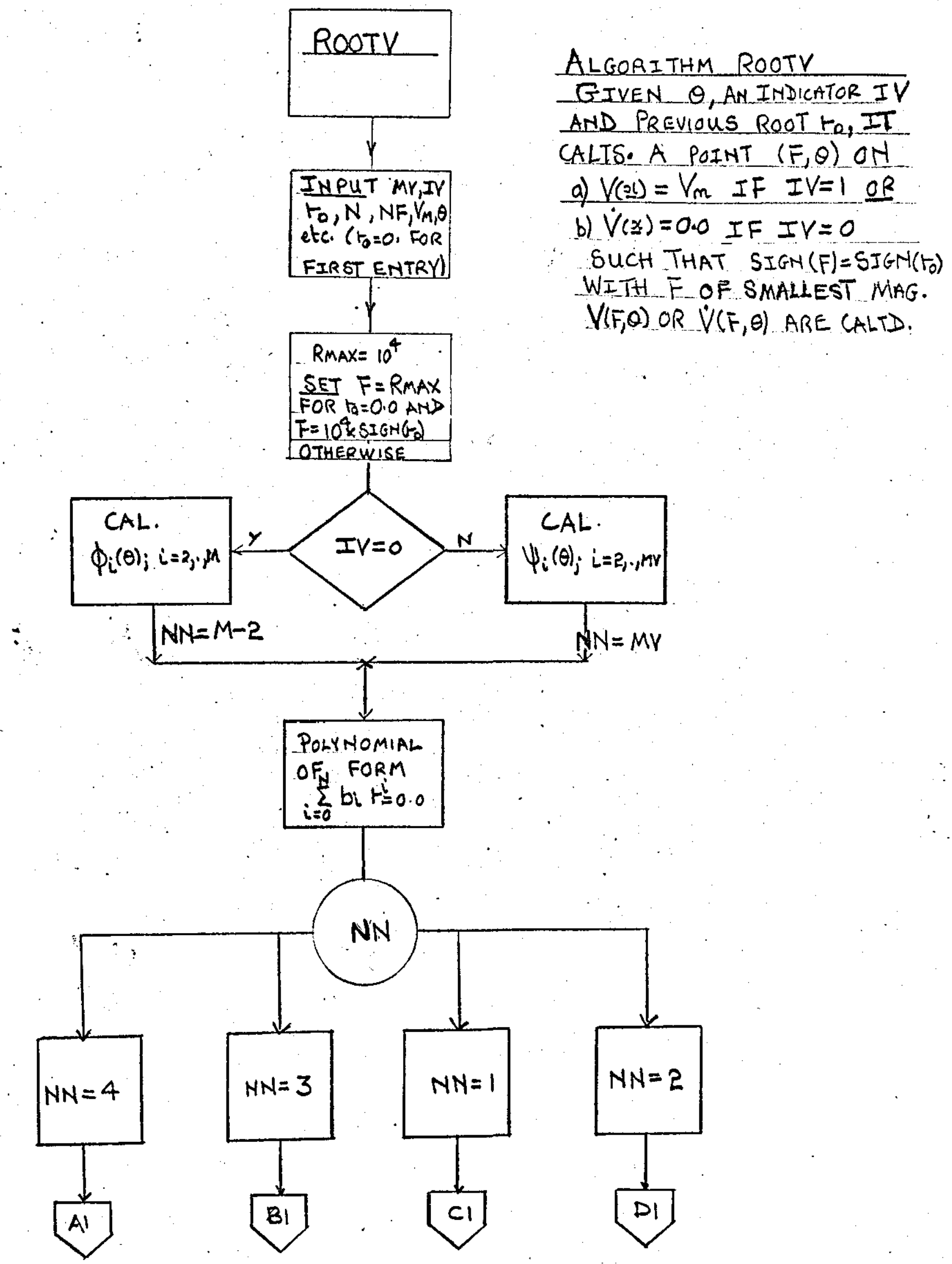

FIG.4.12 (P.T.0) 

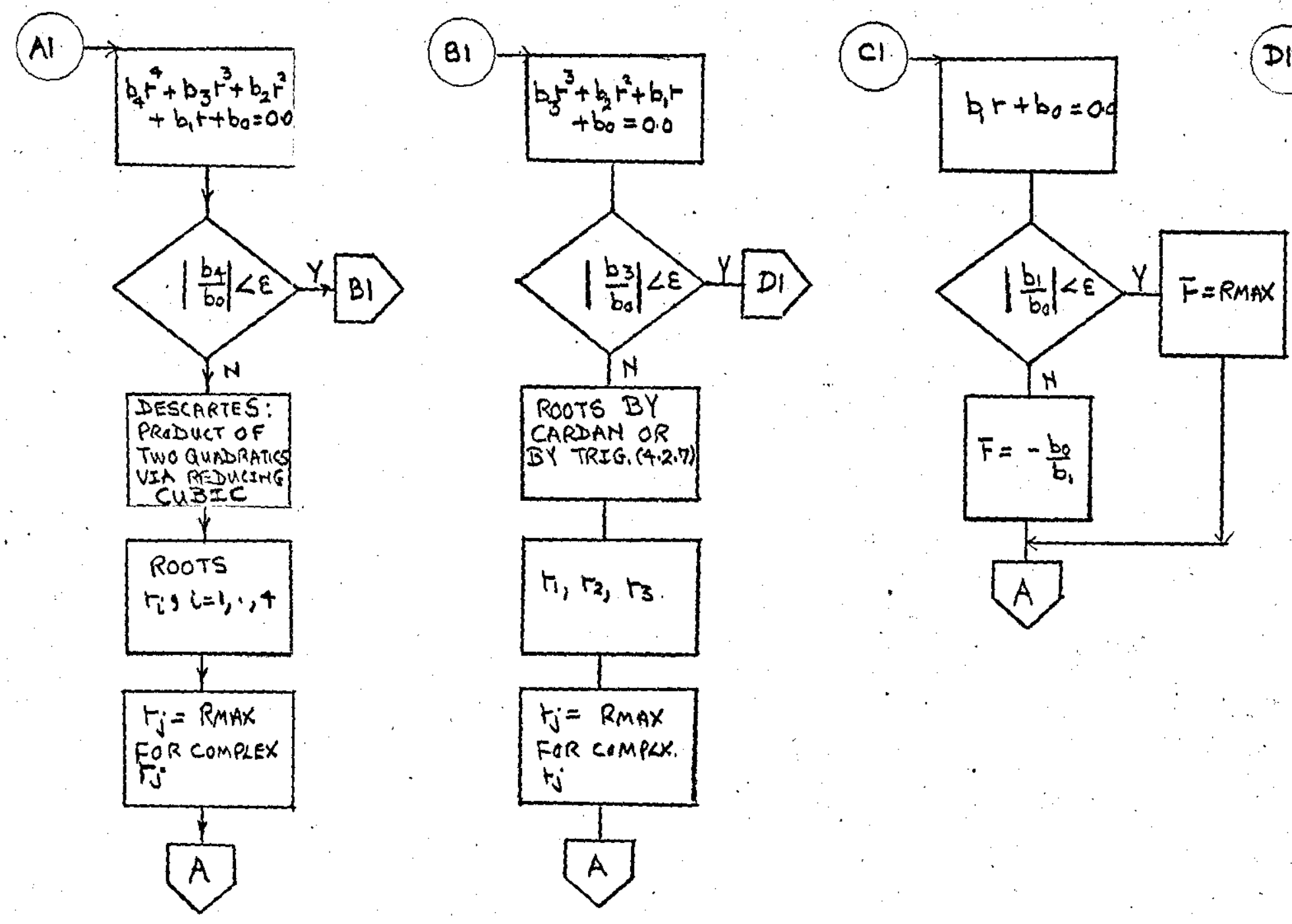

FIG. 4.12 (CONTD.)

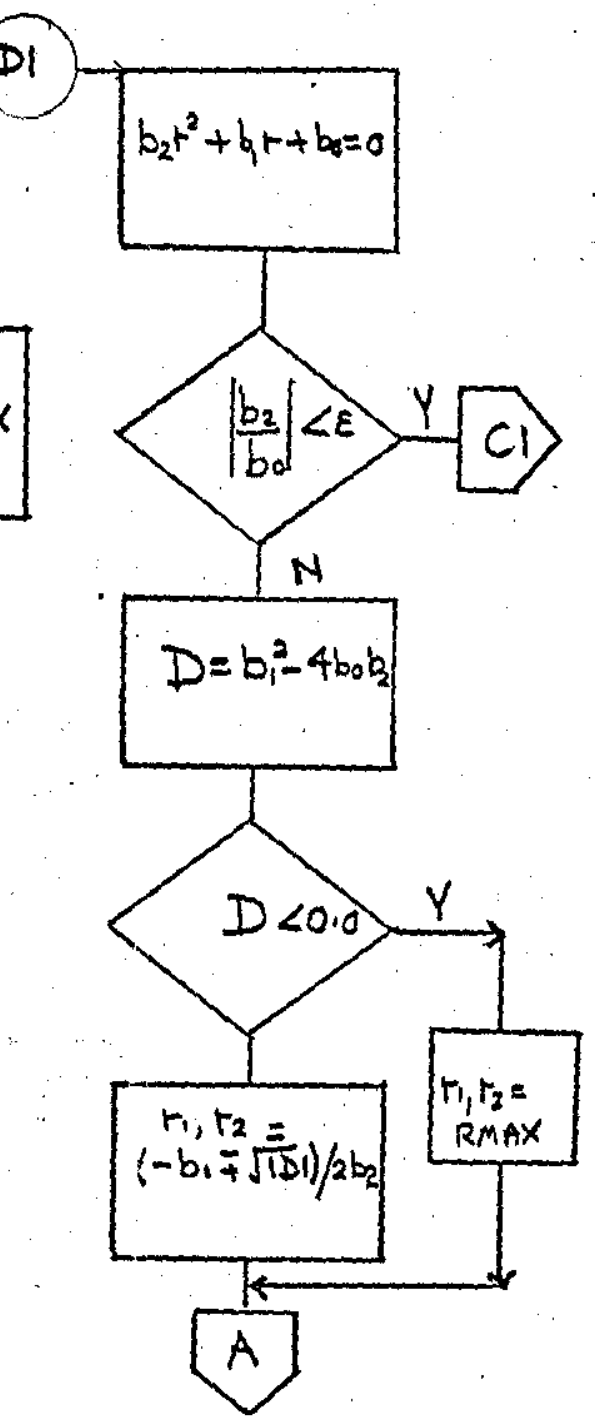




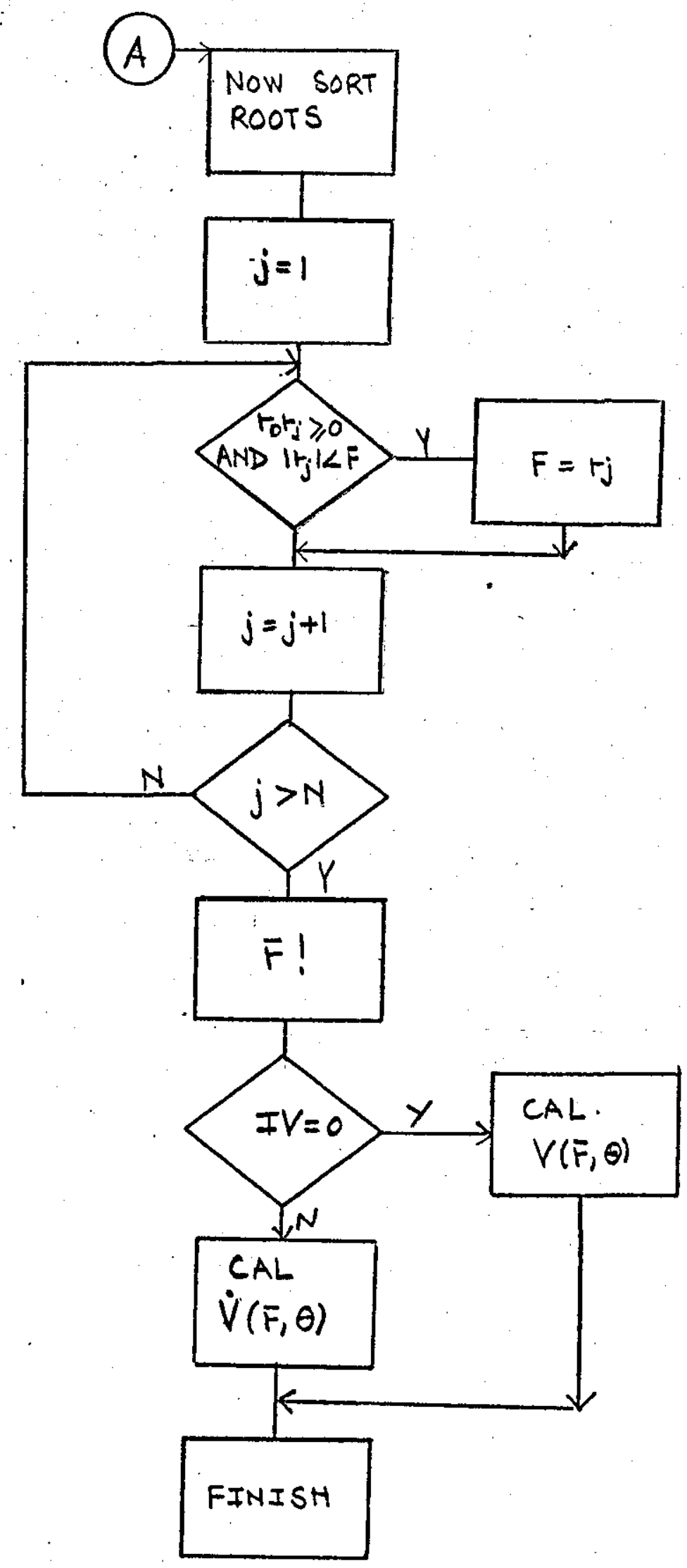

FIG. 4.12 (CONTD.) 


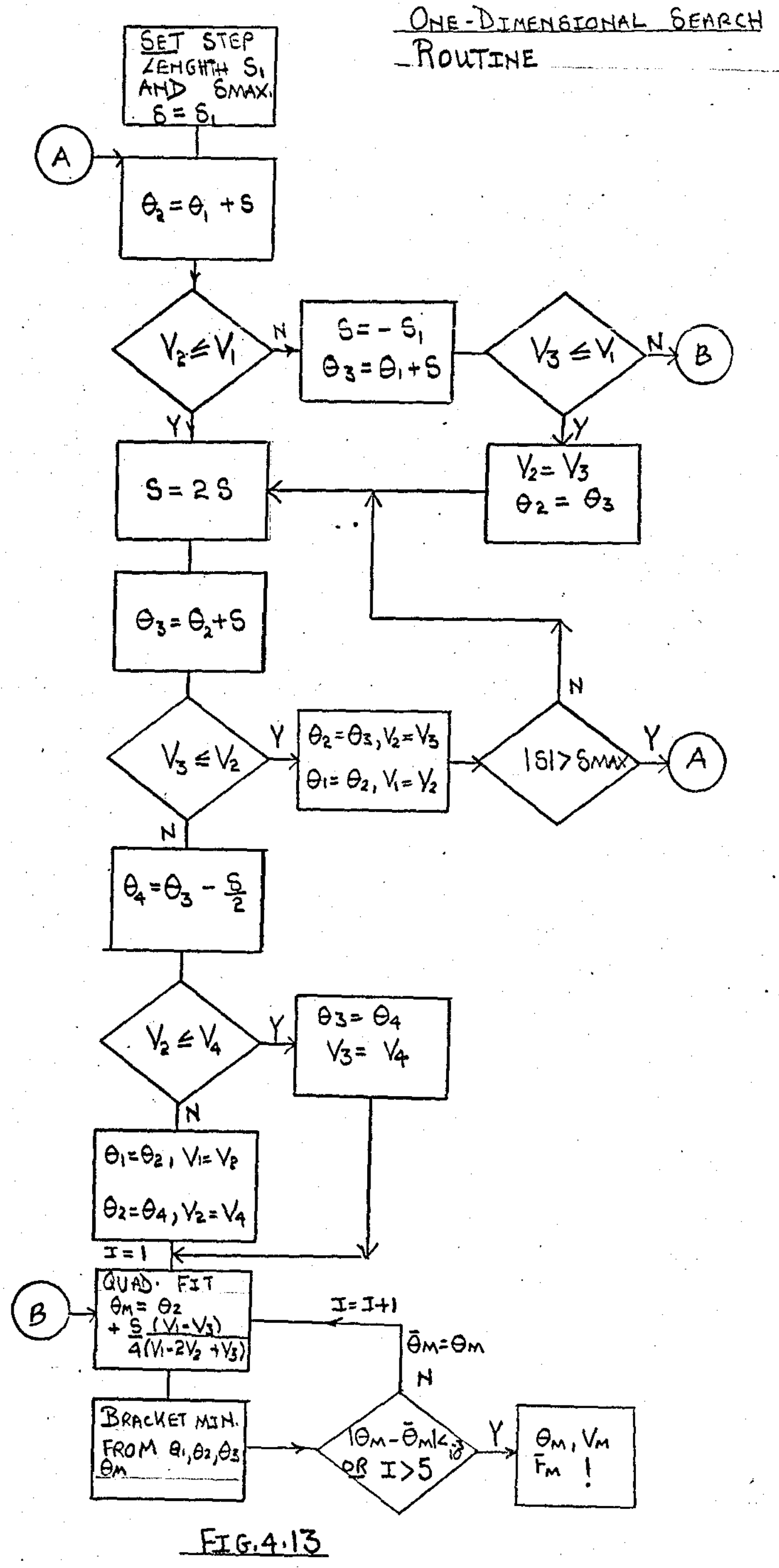




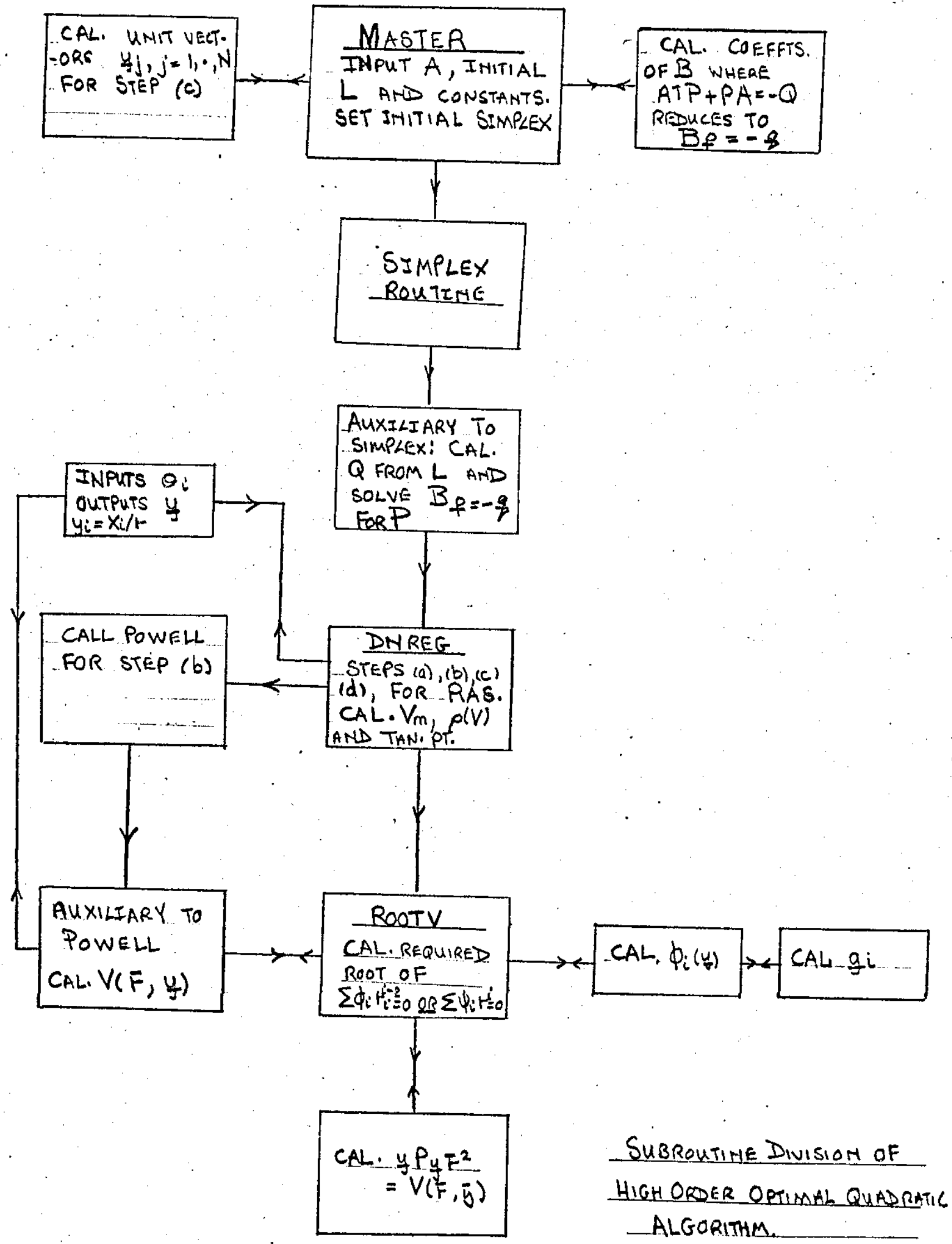

FIG 4.15 
101
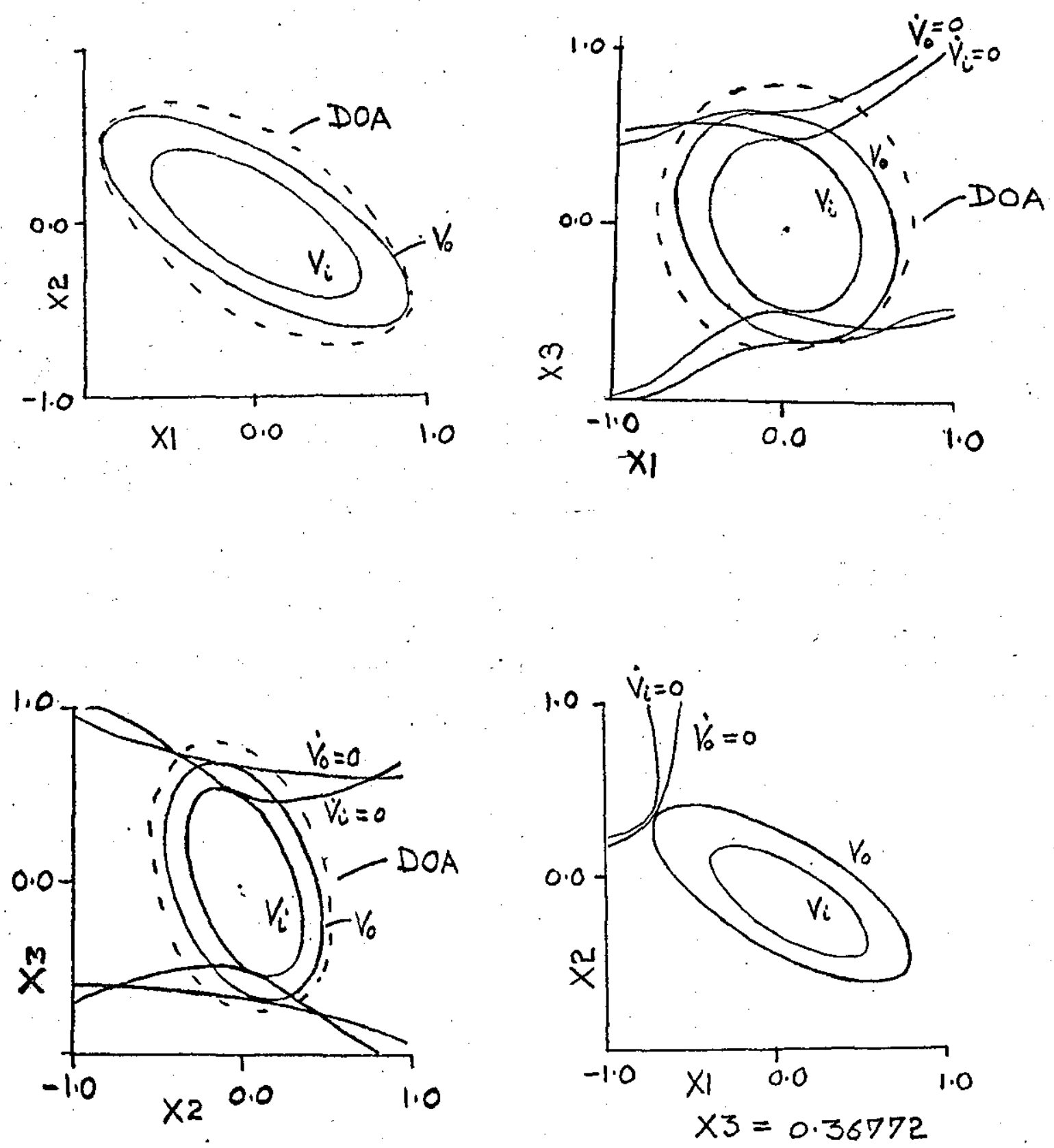

SYSTEM SO

$V_{i}, V_{0}$ initial and final bras Boundaries

FIG 4.16 

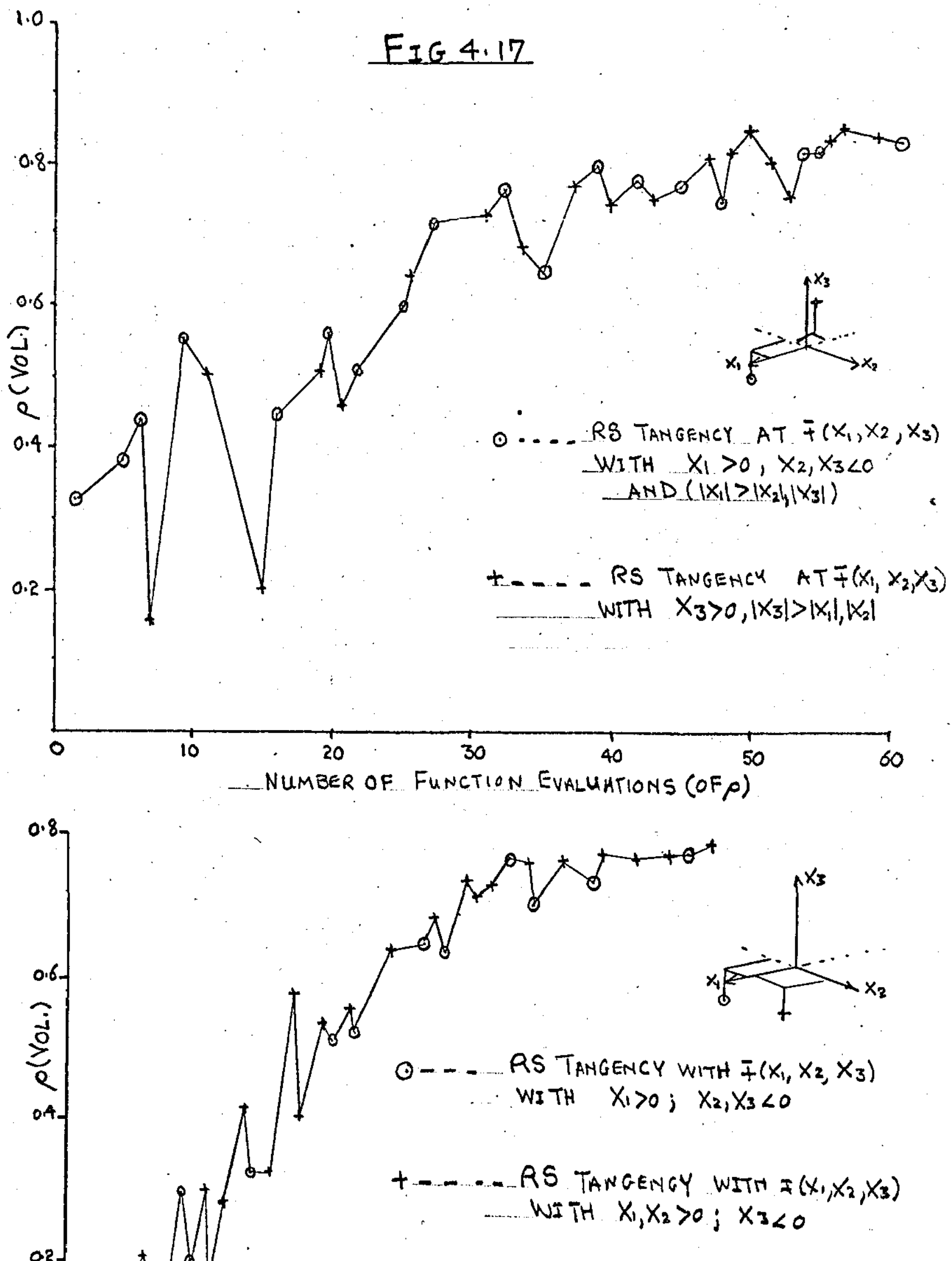

FIG 4.19 

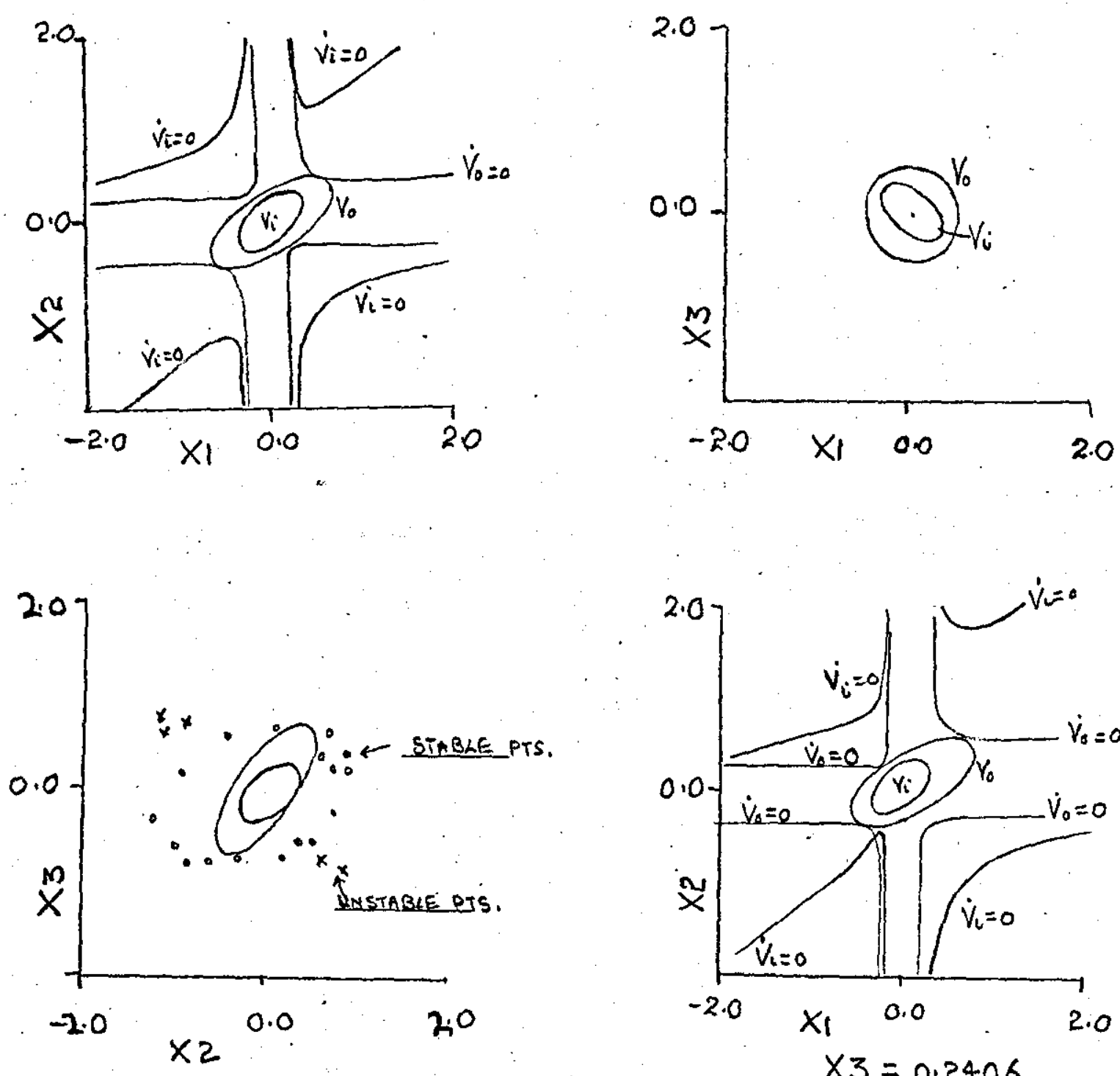

PTS. $0, x$ DETERMINED BY R. VUTTA.
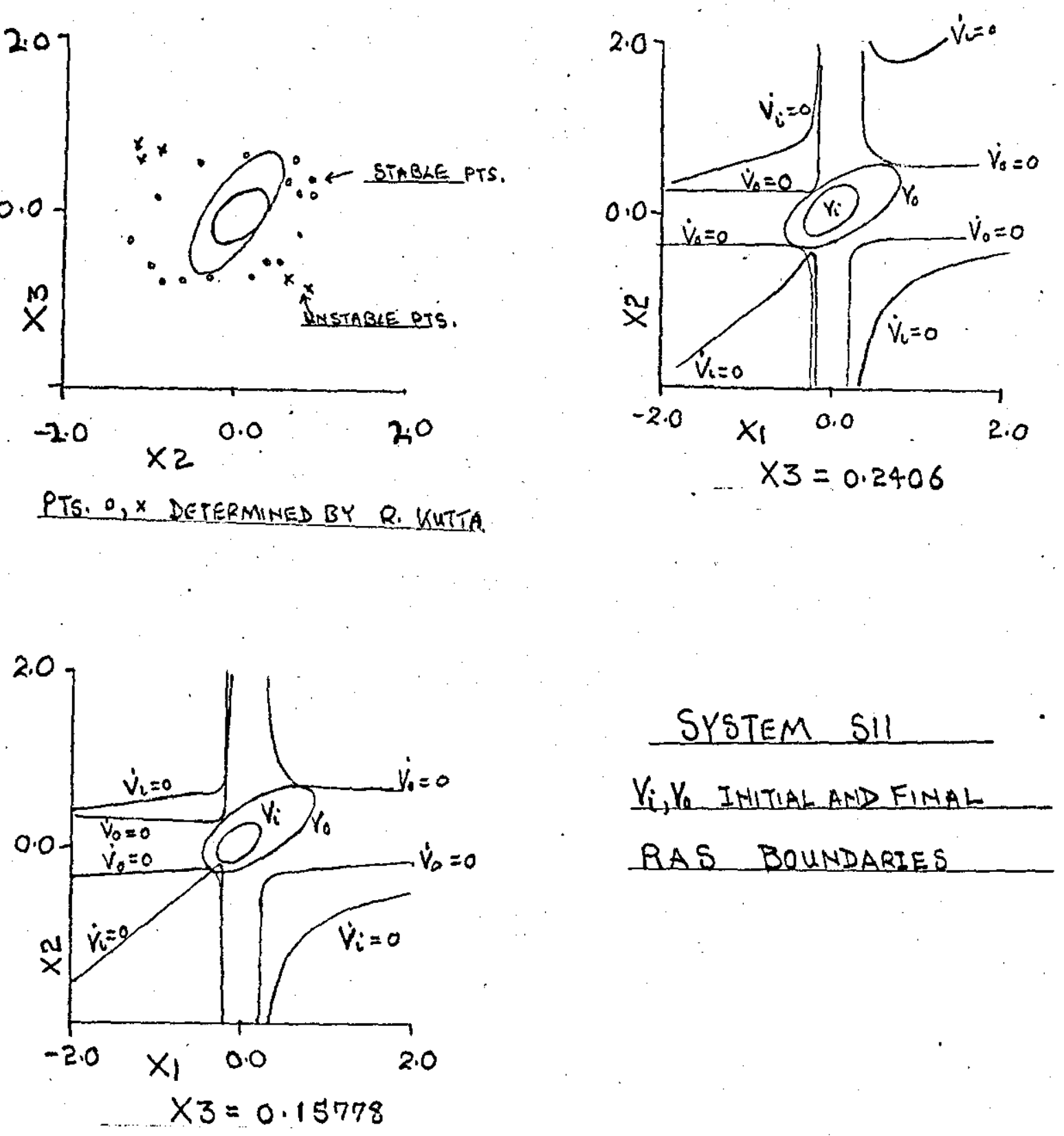


\section{CHAPTER 5}

COMPUTATIONAL MIETHODS FOR OPTIMAL

QUADRATIC AND RAS DETERMINATION

FOR GENERAL NON-IINEAR SYSTIMS. 


\section{Chapter 5}

Computationa1 Methods For Optimal Quadratic and RAS

Determination For General Non-linear Systems.

\subsection{Introduction}

In this chapter two algorithms are proposed. The first is a method for optimal quadratic determination which does not rely on penalty functions as in Geiss (51) or tangency between hypersurfaces as in Rodden (3). It is somewhat heuristic and is based on an idea of Davidson and liurak (47) who have developed a method which uses the special properties of a quadratic. The second is a method which determines an RAS for a general quadratic Lyapanov function via a penalty function approach. It autonatically finds the valid tangency point to a desired accuracy.

\subsection{An Optina1 Quadratic Algorithm}

\section{Development}

The method deals with the system

$$
\underline{x}=\underline{f}(\underline{x}) \quad(\underline{f} \in E) \quad 5.2 .1
$$

Let $A$ be the Jacobian of $\underline{f}(\underline{x})$ at $\underline{x}=0$, assumed stable. Then as in chapter 4 the class of quadratic LF's is determined as $V=\underline{x}^{T} P \underline{x}$, where $P$ solves the matrix equation $A^{T} P+P A=-Q$ for p.d.s. Q. By Theorem 1.5 .3 the region $W$ given by $W:\left(\underline{x} / \underline{T}^{P}<c\right)$, is a stability region for $c$ sufficiently small, the RAS corresponding to $c_{\max }$ Replacing $\mathrm{P}$ by $\mathrm{P} / \mathrm{C}$ the boundary of $\mathrm{W}$ may be written more conveniently as

$$
\underline{x}^{T} P \underline{x}=1
$$


which bounds the volume

$$
\rho=\pi / \sqrt{d}(P)
$$

The $O Q$ problem with respect to $\rho$ is then formulated as

$$
\min d(P)=\prod_{i=1}^{n} \lambda_{I}(P)
$$

subject to the constraints

$$
\begin{array}{lr}
\text { (1) } \mathrm{P} \text { positive def. symmetric } & 5.2 .5 \\
\text { (2) } \underline{\underline{I}}^{\mathrm{T}}(\underline{\mathrm{x}}) \mathrm{P} \underline{\mathrm{x}}<\mathrm{O}, \forall \underline{\mathrm{x}} \in \mathrm{W}, & 5.2 .6
\end{array}
$$

with $W$ now, $W:\left(\underline{x} / \underline{x}^{P} \underline{x}<1, \underline{x} \neq 0\right)$. (Note we have assumed $\|\underline{f}\|=\longrightarrow 0$ as $\|x\| \rightarrow 0)$.

Essentially, $\rho$ is optimized over the p.d.s matrices(P) while constraint (2) ensures that the boundary $\underline{x}^{T} P_{\underline{X}}=1$ is a stability boundary. The main problems are then, the choice of optimization technique and the evaluation of the constraints in (1) and (2).

\section{Constraint Bvaluation}

Since any p.d. $\mathrm{P}$ is expressible as $\mathrm{P}=\mathrm{L}^{\mathrm{T}} \mathrm{L}$ where $\mathrm{L}$, $\mathrm{d}(\mathrm{L}) \neq 0$, is an upper triangular matrix, constraint (1) is avoided by optimizing $\rho$ over L. Constraint (2) can be replaced by

$$
\dot{\mathrm{v}}_{\mathrm{M}}(\mathrm{p})<0
$$

where

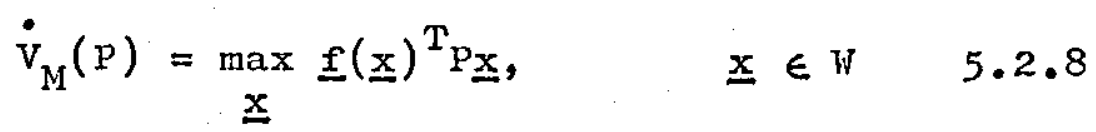

In order to evaluate the latter the following approximation is made: store pre-determined unit vectors, $Y_{j}, j=1,2, \ldots, N_{n}$, which ideally cut a closed surface 
$\left(\underline{x}^{T}{ }^{P} \underline{x}=1\right.$ or $\underline{x}^{T} \underline{x}=1$ say) at equally spaced points. A point on the surface $\underline{x}^{T} \underline{x}=1$ is then given by

$$
\frac{y_{j}}{\left\|L_{Y_{j}}\right\|}=\frac{y_{j}}{\sqrt{Y_{j}^{2} Y_{j}}} ; j=1, \ldots, N_{n}
$$

Hence

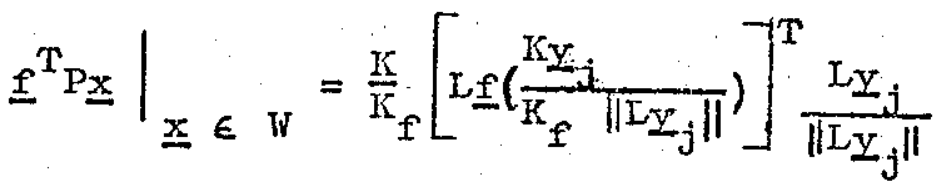

$$
\begin{aligned}
& j=1, \ldots, N_{n} \\
& \mathrm{~K}=1 ; 2, \ldots \mathrm{K}_{\mathrm{f}} \text {, }
\end{aligned}
$$

where $K_{f}$ is the number of grid points occuring along the straight line between $\underline{x}=0$ and $\underline{Y}_{j} /\left\|L_{y_{j}}\right\|$. Although $K_{f}=5$ was chosen by Davidson (see later), it was found sufficient only to evaluate $\dot{V}$ on the surface $\underline{x}^{T}{ }_{P}=1 \quad\left(K_{f}=1\right)$, mainly because of the starting procedure that follows. In short, the problem is now

$$
\min _{L} d(L) \quad(i . e \cdot \max \rho) \quad 5.2 .9
$$

subject to

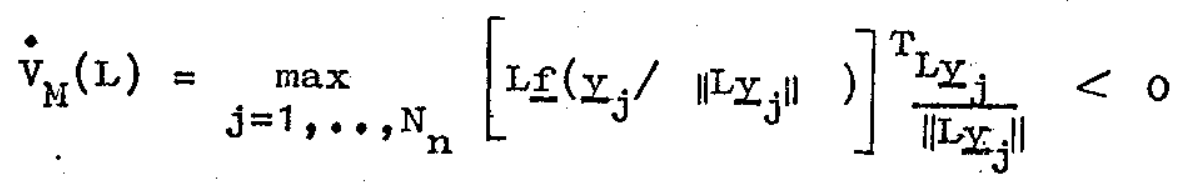

$$
5.2 \cdot 10
$$

provided the initial boundary, $\|$ Lx $\|^{2}=1$, is a stability boundary. (The situation $d(L)=0$ is rarely encountered, but $\mathrm{P}=\mathrm{L}^{\mathrm{T}} \mathrm{L}+\epsilon \mathrm{I}$ can always replace constraint $\left.(1)\right)$. The main points of the algorithm are as follows : 


\section{(a) Choice of Minimization Routine}

Box's (55) Complex method is used to minimize d(I) subject to $\dot{V}_{M(L)}<0$, and the variation used is given in A4. The method is simple to program and often works well for non-convex constraints.

(b) Initial L

In the absence of any other quadratic a natural choice is to choose $Q=I$ and solve

$$
A^{T} P_{1}+P_{1} A=-Q
$$

for $\mathrm{p}_{1}$. For many practical systems this often leads to an initial boundary, $\underline{x}^{T} p_{1} x=\epsilon>0$, being extremely eccentric which implies that the components of $\dot{V}=0$ are usually long thin surfaces. The approximation in 5.2.10 is then only accurate with an extremely fine mesh and computation time is excessive. In this case the choice $P_{1}=\left(S S^{*}\right)^{-1}$ is made from 2.2 .2 where $S$ has unit column vectors.

\section{(c) Initial Boundaxy}

If $P_{1}$ is factored as $P_{1}=L_{1}{ }^{T} L_{1}$ then

$$
\underline{x}^{T} P_{1} \underline{x}=\left\|L_{1} \underline{x}\right\|^{2}=I_{0} \quad\left(I_{0}=10^{-5} \text { say }\right)
$$

$$
5.2 .12
$$

gives a stability boundary $(\dot{\mathrm{V}}<0$ for $\|\underline{x}\|<\epsilon, \epsilon$ smal1). The vectors $\frac{y_{j}}{\left\|L_{j}\right\|}$ are calculated and stored

in $\mathrm{y}_{j}\left(j=1,2, \ldots, \mathrm{N}_{n}\right)$. A one-dimensional search is now made on 1 , starting with $1=1_{0}$, so as to 1 increase the initial boundary in 5.2.12: 


$$
\dot{V}_{M}(1)=\max _{j=1, \ldots, N_{n}}\left[L_{1} £\left(\sqrt{1} y_{j}\right)\right] T_{L_{1} Y_{j}}<0
$$

In practice a simple bisection method was adequate for maximizing 1 such that if finally $1_{2}$ and $l_{3}$ are bracketing values $-\dot{\mathrm{V}}_{\mathrm{I}}\left(1_{2}\right)<0$ and $\dot{\mathrm{V}}_{\mathrm{M}}\left(1_{3}\right)>0-$ and

$$
\left|\frac{1_{3}-I_{2}}{I_{2}}\right|<\text { er } \quad 5.2 .14
$$

the required 1 was obtained.

In detail the steps are :

(1) put $1_{1}=1_{0}\left(\right.$ if $\dot{V}_{M}\left(1_{0}\right)>0$ reduce $1_{0}$ further $)$ and with suitable $s$ put $I_{2}=1_{1}+s$

(2) If $\dot{\mathrm{V}}_{\mathrm{M}}\left(1_{2}\right)<0, s=2 \mathrm{~s}, 1_{1}=1_{2}$ and $I_{2}=1_{1}+\mathrm{s}$ are made and $(2)$ is repeated until $\dot{V}_{M}\left(I_{2}\right)>0$ (If $s>s_{\max }$, an upper bound, repeat (1) with larger s)

(3) then select $s=s / 2, I_{3}=1_{1}+s$ and if $\dot{\mathrm{V}}_{\mathrm{M}}\left(1_{3}\right)<0$ put $1_{1}=1_{3}$, otherwise $1_{2}=1_{3^{\circ}}$ Repeat (3) until 5.2.14 is satisfied. (Typically, $\mathbf{s}=.1, \mathbf{s}_{\max }=10.0$, er $=.3$; the latter being sufficiently large to give a good iaitial complex). The final boundary is $\underline{x}^{T}{ }^{T}=1_{2}$ or $\underline{x}^{T} \underline{x}=1.0$ with $p=p_{1} / 1_{2}\left(I_{2}\right.$ corresponds to $1_{1}$ in step (3))

(d) Choice of Unit Vectors

(1) A simple choice is the set of vectors from the origin to equally spaced points on the unit n-sphere via polar co-ordinates in 4.5 .1 .

(2) For highly eccentric surfaces it is advisable to transform $\underline{x}^{T} P_{1} x=1$ to $\sum \lambda_{i} \underline{z}_{i}^{2}=1$ via an orthogonal transformation $\underline{x}=T_{\underline{z}}\left(T^{T}=T^{-1}\right)$ with $T=$ 
and $\underline{z}_{i}^{T} \underline{z}_{i}=1$. Form an n-dimensional box with axes along $\underline{z}_{i}$ and sides of lengths $2 / \sqrt{\lambda_{i}\left(P_{1}\right)}$. Divide its surface into an equally spaced mesh, then the points give the required vectors when normalized. (Note that the initial vectors need not necessarily be unit vectors).

\section{(e) Initial Complex}

The initial boundary, $\underline{x}^{T_{x}}=1,($ in $(c)$ ) gives an initial feasible point

$$
\mathrm{p}_{\mathrm{o}}=\left(\mathrm{t}_{1}, \mathrm{t}_{2}, \ldots, \mathrm{t}_{\mathrm{m}}\right)
$$

where $m=n(n+1) / 2$ - the number of elements of $L, \quad P=L^{T} L$ and $L$ is written as

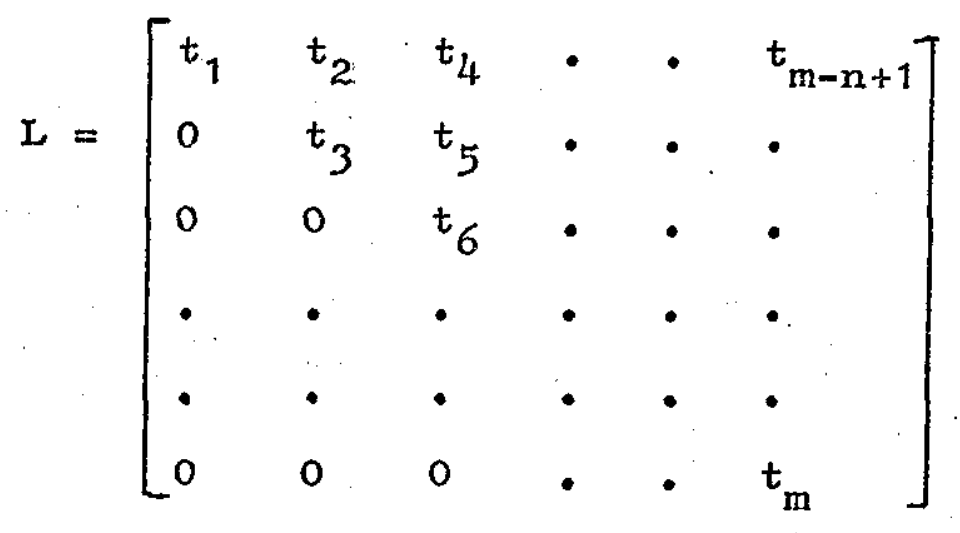

A complex of $2 m+1$ points is used, the remaining $2 \mathrm{~m}$ feasible points being obtained in the folloring way. :

Introduce an $m \times 2 \mathrm{~m}$ matrix $\mathrm{D}$, partitioned as $D:\left(I_{m}, D_{1}\right)=\left(\underline{d}_{1}, \underline{d}_{2}, \ldots, \underline{d}_{2 m}\right)$ with $I$ the $m x$ mit matrix and $D_{1}$ a matrix whose columns, $\underline{d}_{i}$ $(i=m+1, \ldots, 2 m)$, have random elements of zeros or ones. Let $s_{0}=h \max _{i}\left|t_{i}\right|$, then a tentative $j^{\text {th }}$ feasible point, $\underline{p}_{j}(j=1, \ldots, 2 \mathrm{~m})$, is determined as

$$
p_{j}=p_{0}+\underline{d}_{j} s, \quad s=s_{0}
$$


If $\dot{\mathrm{V}}_{\mathrm{M}}\left(\mathrm{p}_{j}\right)<0$ from 5.2 .10 , the point $p_{j}$ is 'reasible' and is accepted, but if $\dot{V}_{M}\left(p_{j}\right) \geq 0$ we find $\dot{\mathrm{V}}_{\mathrm{M}}\left(\mathrm{p}_{\mathrm{j}}^{*}\right)=\dot{\mathrm{V}}_{\mathrm{M}}\left(\mathrm{p}_{\mathrm{o}}-\mathrm{d}_{\mathrm{j}} \mathrm{s}\right)$. If $\dot{\mathrm{V}}_{\mathrm{M}}\left(\mathrm{p}_{\mathrm{j}}^{*}\right)<0, \mathrm{p}_{\mathrm{j}}^{*}$ is accepted, otherwise $s$ is halved and the process repeated until a feasible point is found or until $s<.01 h$, say, when the complex is deemed too small ( $h=.2$ was found practical).

It was found that when er was made too small in obtaining the initial boundary, either the resulting initial complex was also very small or that function values at points $\mathfrak{p}_{j}$ were much less than that at $\mathfrak{p}_{0}$. The idea of Box (55) and others of generating random and possibly large $\mathfrak{p}_{j}$, was impractical due to the presence of the equal tangency surface ( $c . f$. Chapter 4). A point lying on a line drawn between two feasible points on opposite sides of this surface was usually infeasible (no matter how close the points).

\section{(f) Other Features}

The unit vectors need only span a half space of $\mathrm{E}^{\mathrm{n}}, \mathrm{x}_{3}>0$ say, for then 5.2 .10 is evaluated as

$$
\dot{v}_{M}(L)=\max _{j=1, \ldots, N_{n}} \mp\left[L L^{f}\left(\mp \frac{Y_{i}}{\left\|L_{y_{j}}\right\|}\right)\right]^{T} \frac{L_{y_{j}}}{\left\|L_{y_{j}}\right\|}
$$

A quick routine is used to calculate Lx, the resulting vector being stored in $x$, thus

$$
x_{i}=\sum_{j=i}^{n} 1_{i j} x_{j}
$$

Also if $\underline{f}(-\underline{x})=-\underline{f}(\underline{x})$ computation of $\dot{V}_{M}(I)$ is halved due to radial symmetry of the contour $\dot{v}(\underline{x})=0$. 
On exit from the Complex minimization the final boundary, $\underline{x}^{T} P_{\underline{x}}=1$, is an approximation to the RAS boundary, $\quad x^{T} P_{X}=V_{M}$. The former boundary is then either verified by using a finer mesh to calculate $\dot{\mathrm{V}}_{\mathrm{M}}(\mathrm{I})$ or the exact RAS is obtained by an algorithm such as that in section 5.4 .

The number of calculations required to evaluate 5.2 .15 is effectively $N_{n}\left(\frac{3}{2^{2}}+n\left(2 c_{1}+11 / 2\right)\right)$ with $c_{1}$ the average number of calculations to evaluate each $f_{i}$. Assuming $N_{n}=c^{n}$ the approximate constraint and optimal quadratic computation time for large $n$ will vary as $c^{n}\left(\frac{n^{2} 3}{2}+2 c_{1} n\right)$ and $\frac{\mathrm{K}_{1} \mathrm{n}^{2} \mathrm{c}^{\mathrm{n}}}{2}\left(\frac{3 \mathrm{n}^{2}}{2}+2 \mathrm{c}_{1} \mathrm{n}\right)$ respectively, the optimization being over $m\left(\leftarrow \mathrm{n}^{2} / 2\right)$ parameters (in practice $5<\mathrm{c}>10)$.

The algorithm has been programmed in Fortran IV and Fig's 5.1 and 5.2 show flow diagrams for the master and connecting segments. (A listing is given in A 5).

\section{Summary of Algorithm}

For completeness we summarize the main steps of the new algorithn along side those of Davidson and Kurak's in which

$$
\dot{v}_{M}(P)=\max _{\substack{j=1, \ldots, N_{n} \\ K=1, \ldots, K_{f}}} \underline{T}^{T}\left(\frac{K y_{j}}{K_{f}{ }^{T} P_{j}^{T} Y_{j}}\right) P_{j}
$$


Step

New

\section{Davidson \& Jurak}

(1) Evaluate $A=\left.\frac{\partial \underline{x}}{\partial \underline{x}}\right|_{\underline{x}=0} \quad$ and solve

$$
A^{T} P_{1}+P_{1} A=-Q \text { for } P_{1} \text { with }
$$
a) $Q=I$
a) $Q=I$

\section{ox}

b) Choose $\mathrm{P}_{1}=\left(\mathrm{SS}^{*}\right)^{-1}$

(2) Starting with $1=1_{0}\left(=10^{-5}\right.$ say) $\max (1)$ subject to

$$
\begin{array}{l|l}
\dot{V}_{M}(1)<0 \\
(5.2 .13)
\end{array} \mid \begin{aligned}
& \dot{V}_{M}\left(P_{1} / 1\right)<0 \\
& (5.2 .16)
\end{aligned}
$$

by bisection method (in (c) by Rosenbrock (in A4)

This gives $\underline{x}^{T} \underline{x}=1$

$P=P_{1} / I_{2}=I_{1}^{T} L_{1} / I_{2}$

This gives $\underline{x}^{T} \underline{x}=1$ with

(3)

$$
\overline{\mathrm{p}}=\mathrm{p}_{1} / 1_{\max }
$$

$\mid \begin{aligned} & \text { Starting with this } \overline{\mathrm{P}} \\ & \text { min } M(\overline{\mathrm{P}}) \text { subject to } \mathrm{m}(\overline{\mathrm{P}})>0 \\ & \text { and } \dot{\mathrm{V}}_{\mathrm{M}}(\overline{\mathrm{P}})<0(5.2 .16)\end{aligned}$

(4) Starting with $\mathrm{L}=\mathrm{L}_{1} /{\sqrt{I_{2}}}_{2}$ $\min d(L)(\max \rho)$

subject to $1_{i i} \neq 0$

and $\dot{\mathrm{V}}_{\mathrm{M}}(\mathrm{L})<0$

by the Complex

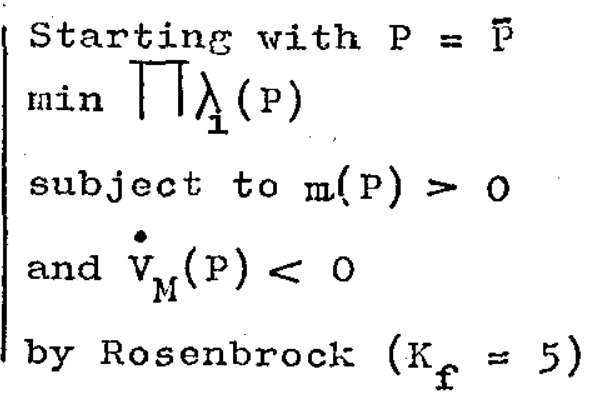

optimization routine (A4)

(5) Verify $\dot{V}<0$ in $W:\left(\underline{x} \underline{x}^{T} \underline{x}<1, \underline{x} \neq 0\right.$ ) by evaluating

a) $\dot{V}_{M}(I)$ with fine mesh

a) $\dot{v}_{M}(P)$ with fine mesh

\section{or}

b) exact RAS (Section 5.4)

Steps (3) and (4) of Davidson's alrorithm require the evaluation of the eigenvalues of $P$ (Jacobi's method used). 


\subsection{Numerical Results}

Table 5.1 shovs details of a comparison of the nev method with that of Davidson for the following systems : System $\$ 13(\mathrm{Yu}(6))$

Consider the following equations for a synchronous generator

$$
\begin{aligned}
& \dot{\mathrm{x}}_{1}=\mathrm{x}_{2} \\
& \dot{\mathrm{x}}_{2}=-\mathrm{R}-\mathrm{D \textrm {x } _ { 2 }}
\end{aligned}
$$

$$
\text { where } \quad \begin{aligned}
D & =0.0372+0.152 \cos \left(2 x_{1}+1.7744\right) \\
\text { and } \quad R & =\sin \left(x_{1}+.8872\right)-\sin (.8872) \\
& -.1291\left(\sin \left(2 x_{1}+1.7744\right)-\sin (1.7744)\right)
\end{aligned}
$$

With $Q=I, \quad I_{0}=10^{-5}$ and $\mathbb{N}_{2}=30$ the initial boundary was expanded to

$$
24.71 x_{1}^{2}+1.4524 x_{1} x_{2}+36.1 x_{2}^{2}=1.1
$$

Pig 5.3 shows property $A$ is present with the final boundary

$$
11.477 x_{1}^{2}+.928 x_{1} x_{2}+17.02 x_{2}^{2}=1.0
$$

having two noints of contact with its constraint contour. The RAS is not a good estimate of the DOA. System S14 (Hewit (2))

The system is a variation of a surge-tank system found in Hevit

$$
\begin{aligned}
\dot{x}_{1} & =x_{2} \\
\dot{x}_{2} & =-x_{1}\left(1-a\left(2+x_{1}\right)\right) /\left(1+x_{1}\right)^{2} \\
& -\frac{a}{b} x_{2}^{2}-\frac{b}{a} x_{2}\left(2 \frac{a}{b}\left(1+x_{1}\right)-1\right) /\left(1+x_{1}\right)^{2} \\
a & =.2, b=.075
\end{aligned}
$$

Fig 5.4 shows the oQ boundary has not two but three points of contact with its constraint contour, the initial quadratic having only one. ( $i . e$ the $O Q$ has 3 valid tan.pts). 
TABLI 5.1

\begin{tabular}{|c|c|c|c|c|c|c|}
\hline \multicolumn{3}{|c|}{$\begin{array}{l}D=\text { DAVIDSON \& KURA } \\
\text { TrOR D : } h=.2, I_{0}=10^{-5}\end{array}$} & , ex=0.3 & $\begin{array}{l}C=\mathrm{NEW} C \mathrm{CC} \\
\text { FOR } \mathrm{C}: 1_{0}=\end{array}$ & $\begin{array}{l}\text { ONPLEX METH } \\
=10^{-5}, \mathbf{z}=\end{array}$ & $-1.0\left(A^{4}\right)$ \\
\hline SYSTEM & $\begin{array}{l}\text { INITIAI } \\
P \text { OR } Q\end{array}$ & $N_{n}$ & $\begin{array}{l}\text { NITIAI } \\
\text { VoI }\end{array}$ & $\begin{array}{l}\text { FINAL } \\
\text { VOL }\end{array}$ & $\begin{array}{l}\text { NO. OF } \\
\text { CONETANAN } \\
\text { EVALUATIONS } \\
\left(\dot{\mathrm{V}}_{\mathrm{M}}(\mathrm{L})\right)\end{array}$ & $\begin{array}{l}\text { TIHE IN } \\
\text { MILL/SEC. }\end{array}$ \\
\hline s13 $: \begin{array}{l}\mathrm{D} \\
\mathrm{C}\end{array}$ & $Q=I$ & $\begin{array}{l}30 \\
n\end{array}$ & 0.116 & $\begin{array}{l}0.22104 \\
0.2249\end{array}$ & $\begin{array}{l}688 \\
360\end{array}$ & $\frac{350}{120}$ \\
\hline $\begin{array}{ll}\mathrm{S14} & \mathrm{D} \\
\mathrm{C} & \end{array}$ & $Q=I$ & $\frac{30}{i t}$ & $\frac{0.69 \times 10^{-}}{1}$ & $\left\{\begin{array}{l}3.169 \times 10^{-3} \\
1.179 \times 10^{-3}\end{array}\right.$ & $\begin{array}{r}332 \\
420\end{array}$ & $\begin{array}{l}280 \\
160\end{array}$ \\
\hline $\begin{array}{c:c}\mathrm{S} 15 & \mathrm{D} \\
& \mathrm{C} \\
& \mathrm{C} \\
& \\
\end{array}$ & $\begin{array}{r}Q=I \\
\text { RESTART }\end{array}$ & $\begin{array}{c}180 \\
n\end{array}$ & $\begin{array}{r}0.516 \\
1 \\
2.85\end{array}$ & $\begin{array}{l}0.960 \\
2.85 \\
3.075\end{array}$ & $\begin{array}{r}1300 \\
1400 \\
460\end{array}$ & $\begin{array}{r}2000 \\
1220 \\
780\end{array}$ \\
\hline $\begin{array}{ll}\mathrm{S16} & \mathrm{D} \\
& \mathrm{C} \\
& \mathrm{C} \\
\end{array}$ & $\begin{array}{c}Q=I \\
"\end{array}$ & $\begin{array}{r}180 \\
370 \\
\end{array}$ & $\begin{array}{r}3.5 \\
3.5 \\
\end{array}$ & 25.0 & $\begin{array}{r}1370 \\
800\end{array}$ & $\begin{array}{l}2000 \\
2000\end{array}$ \\
\hline $\begin{array}{ll}S 11 & D \\
& c\end{array}$ & $\begin{array}{c}\mathrm{P}=\left(S S^{*}\right) \\
\|\end{array}$ & $\begin{array}{c}180 \\
n\end{array}$ & .104 & $\begin{array}{l}0.26313 \\
0.383\end{array}$ & $\frac{1230}{1300}$ & $\frac{3000}{3000}$ \\
\hline $\mathrm{D}$ & $Q=I$ & 180 & 0.10 & 0.146 & 1200 & 2900 \\
\hline c & $"$ & $"$ & .1 & 0.285 & 800 & 1500 \\
\hline$c$ & $\mathrm{P}=\left(\mathrm{Ss} \mathrm{s}^{*}\right)$ & 370 & 0.104 & 0.376 & 900 & 1900 \\
\hline s17 $\mathrm{C}$ & $\mathrm{P}=\left(\begin{array}{l}\mathrm{S} \\
\mathrm{S}\end{array}\right)$ & 1240 & $.12 \times 10^{-3}$ & $165 \times 10^{-3}$ & 1040 & 5000 \\
\hline
\end{tabular}




\section{System $\$ 15$}

A system having a fixed constraint contour, $x^{T}{ }^{T}=1$, is

$$
\underline{x}=-\underline{x}+\left(\underline{x}^{T}{ }_{B x}\right) \underline{x}
$$

with DOA, $\underline{x}^{T} \mathrm{~B} x<1$. We have chosen

$$
B:=\left[\begin{array}{lll}
1 & 1 & 0 \\
1 & 2 & 1 \\
0 & 1 & 2
\end{array}\right]
$$

Step (3) of Davidson's algorithm is redundant since $P=I$ with $Q=2 I$ and Table 5.1 exhibits the poor convergence of the algorithm. In fact the new algorithm was found superior to the latter for all third order systems tried.

A reason for the inferiority of Davidson was the lack of convergence of Rosenbrock's routine on the. constrained problem. As the volume increased it was found that values of $P$ were reached, exhibiting an equal tangency surface, where a large amount of time was spent by the search directions oscillating between the boundary zones, determined by Rosenbrock's penalty function (Appendix 4), and the feasible region $\dot{V}_{M}(P)<0$. (Box (55) has noted. this phenomenon).

The new Complex method gave better convergence although it seemed to stick near the optimum. Closer inspection showed that the vector $\mathrm{y}_{j}$, giving $\dot{\mathrm{v}}_{\mathrm{M}}(\mathrm{L})$, alternated between two or more places on the constraint contour. After restarting the procedure a final volume of $\rho=3.07$ was obtained with

$$
P=\left[\begin{array}{rrr}
1.035 & 1.028 & -0.009 \\
1.028 & 2.025 & 0.992 \\
-0.009 & 0.992 & 2.003
\end{array}\right]
$$

(For the $O Q$ boundary, $\underline{x}^{T}{ } \underline{x}=1, \rho=\pi$ ). 


\section{System $\$ 16$}

The system is that of 54 (Chapter 4) with $a_{1}=1$, $a_{2}=2$ and $a_{3}=3$. Again the new method is superior. The best volume achieved by the third order algorithn of chapter 4 was $\rho=68.01$ which is superior to the results in Table 5.1 .

System $11(\mathrm{c} 4)$

For this system a lack of convergence was found for both methods which was attributed to the presence of the equal tangency surface, with two RS components of the constraint contour. For the new algoritm, whatever the initial $\mathrm{L}_{1}$ ( or $\mathrm{P}_{1}$ ), a point near this surface was obtained which tended to shrink the complex of points so that further progress was poor.

Step (3) of Davidson's method 'of minimizing $M(\bar{p})$, originally intended to make the initial boundary less eccentric and give a good starting $P$ for the final minimization of step (4), proved unsatisfactory. In many cases the procedure gave a smaller starting volume. Infact, Davidson (4) gives the $O Q$ boundary as

$$
\underline{x}^{T} P=\underline{x}^{T}\left[\begin{array}{ccc}
12.5 & -8.1 & 3.0 \\
\cdot & 20.8 & -8.5 \\
. & . & 13.4
\end{array}\right]=1.0
$$

which is incorrect (the RAS for this $p$ satisfies $x^{T} P_{x}<10^{-4}$ ) and results from the poor initial $P$ from step (3). We note that the boundary $\underline{x}^{T}{ } \underline{x}=1$ can be made less eccentric, more effectively, by minimizing $\mu(P)=M(P) / m(P)$, but in so doing the corresponding $Q$ may approach a positive semidefinite matrix with an invalid LF. However, a system where the procedure is useful is the following approximation to a relay system 


$$
\begin{aligned}
& \dot{x}_{1}=x_{2} \\
& \dot{x}_{2}=-\tanh \left(100\left(x_{1}+x_{2}\right)\right)+x_{2}
\end{aligned}
$$

with

$$
A=\left[\begin{array}{cc}
0 & 1 \\
-101 & -99
\end{array}\right]
$$

The initial (expanded) boundary for $Q=I$ is

$$
\underline{x}^{\mathrm{T}}\left[\begin{array}{cc}
1.005 & 0.0049 \\
0 & 0.00504
\end{array}\right] x=2.55 \times 10^{-5}
$$

which i.s very eccentric. After 970 constraint evaluations of $\dot{V}_{M}(\bar{P})$ in minimizing $M(P)$ we have

$$
\overrightarrow{\mathrm{p}}=\left[\begin{array}{cc}
4.96 & 1.30 \\
. & 2.42
\end{array}\right]
$$

However the choice $\mathrm{P}=\left(S \mathrm{~S}^{*}\right)^{-1}$ gives

$$
P=\left[\begin{array}{ll}
1.516 & .5413 \\
.5413 & .4844
\end{array}\right]
$$

which is a good initial estimate of the optimal $P$ with boundary

$$
\underline{x}^{T}\left[\begin{array}{cc}
4.81 & 1.33 \\
. & 2.41
\end{array}\right] \underline{x}=1.0
$$

System $\$ 17$ (Rao (5))

As a final example consider the system of a synchronous machine swinging against an infinite busbar

$$
\begin{aligned}
& \dot{x}_{1}=x_{2} \\
& \dot{x}_{2}=28.61-84.99\left(b+x_{3}\right) \sin \left(x_{1}+a\right) \\
& \quad+21.53 \sin 2\left(x_{1}+a\right) \\
& \dot{x}_{3}=0.36-0.621\left(b+x_{3}\right)+.421 \cos \left(x_{1}+a\right)
\end{aligned}
$$

The quantities $a$ and $b$ result from a shift of origin (solutions of $\dot{x}_{1}=\dot{x}_{2}=\dot{x}_{3}=0$ ) and Rao gives $a=0.478$ 
$b=1.18$ for the stable singularity. As seen in Table 5.1 convergence is very slow and no reasonable $O Q$ has been obtained. Two important points however, are raised :

(a) that an accuracy of $10^{-3}$ in cletermining $a$ and $b$ is insufficient, giving an invalid boundary for $1_{0}=10^{-5}$ when infact the boundary is valia and

(b) that spurious quadratics were obtained with $\mathrm{N}_{3}=370$ due to inadequate mesh size, the $\dot{V}=0$ contours being long thin surfaces.

The defects were remedied by more exact calculation of a and $b(a=.4779930, b=1.1816655)$ and the choice $N_{n}=1240$, with large increase in computing time. Sections of the best quadratic boundary through the co-ordinate planes and the tangency point $(0.0327,0.0229,-.0483)$ are shown in Fig 5.5 The RAS is poor.

\section{Comment}

The application of the algorithms described certainly shows that property $A$ and its associated tangency surface are present for varied practical systems and can cause problems in convergence to the OQ. For high order systems the latter also suffers from the high dimensional problem of calculating $\dot{V}_{M}(L)$ and the storage of the unit vectors. (Geiss (51) has taken 24 min. for one RAS determination for a satellite problem with $n=9$ and $m=45$ ). 


\subsection{A Method For Quadratic RAS Deternination For High Ordex}

\section{Systerns}

Given the system 5.2.1 the problem of determining a RAS for a quadratic, $V=\underline{x}^{T}{ }^{2}$, has been formulated as the constrained problem ( $\mathrm{C} 4$ )

$$
\begin{array}{ll}
\min V(\underline{x}), \underline{x} \in E_{v} & 5.4 .1 \\
\mathrm{E}_{\mathrm{v}}:(\dot{\mathrm{V}}=0, \underline{\mathrm{x}} \neq 00) & 5.4 .2
\end{array}
$$

When using a penalty function (PF) approach two main points must be considered :

1) ensuring that the PF, or sequence of PF's, approach a local minimum of 5.4 .1 excluding the trivial solution $\underline{x}=0$;

2) ensuring that, if the minimum point is not a valid tangency point, a sequence of decreasing minima is obtained which eventually give the global minimum, and thus the valid tangency point.

To satisfy these criteria an algorithm has been developed, the main points of which are the following : (a) Choice of Initial LF

Unless a particular RAS is required $P$ is chosen as

$$
P=\left(S S^{*}\right)^{-1}
$$

(b) Initial Starting Point of Minimization

A good initial starting point, $\underline{x}_{0}$, for the $P F$ minimization is found by storing unit vectors as in the complex algorithm and then expanding the boundary in 5.2 .12 until 5.214 is satisfied to a given accuracy $\left(e x=.1\right.$ say). The vector $y_{j}$ giving the maximum value of. $\dot{V}$ on $\underline{x}^{T} P_{x}=1_{2}$ is then taken as $x_{0}$. 


\section{(c) Choice of Penalty Function and Minimization Routine}

To avoid the trivial solution $\underline{x}=0$ the constraint in 5.4 .2 is posed as

$$
\dot{e}(\underline{x})=\dot{v}(\underline{x}) / v^{z}=0 \quad(z=1 \text { or } 2) \quad 5.4 .3
$$

Then a PF from Miele (62) is chosen as

$$
\begin{aligned}
W(\underline{x}) & =V(\underline{x})+\lambda e(\underline{x})+K^{2}(\underline{x}) \\
& =R(\underline{x}, \lambda)+K^{2}(\underline{x})
\end{aligned}
$$

where $\lambda_{\text {is }}$ essentially an approximation to the Lagrange multiplier and $K>0$ a penalty constant. Starting with $x=\underline{x}_{0}$, W is minimized for several cycles by the algorithm of Fletcher and Powell $(60,64)$ of conjugate directions, where a 'cycle' consists of $\mathrm{n}$ iterations of the latter with $\mathrm{K}$ and $\lambda$ kept constant. After each cycle $\lambda$ and $K$ are updated and convergence is obtained when the conditions

$$
|\theta|<\epsilon
$$

and

$$
5.4 .5
$$

$$
\|\underline{\nabla}(x, \lambda)\|<\epsilon
$$

are satisfied (typically $\epsilon=10^{-4}$ ). If however NC, the number of cycles, exceeds some upper bound (NC $>$ NIT say) the minimization is termed a failure and another initial $\underline{x}_{0}$ is sought (see A4 for updating of $\lambda$ and $K$ and Fletcher-Powell routine).

Since the Fletcher-Powell routine requires gradients of $W$ we assume $J(\underline{f})$, the Jacobian of $\underline{f}$, can be suitably calculated. Then for $z=1$

$$
\nabla V=\underline{\nabla V}+\underline{\nabla e}\left(\lambda+2 K_{e}\right)
$$


where $Z e=\frac{1}{V} 2(V \not \dot{V}-\dot{V} \square V)$

and $\quad \dot{\nabla V}=2\left(P_{\underline{I}}(\underline{x})+J(\underline{\underline{f}})^{\top} P_{\underline{x}}\right)$

Prior to the minimization, scale factors for $x, V$ and $e$ are derived $\left(\mathrm{xsc}_{i}, \mathrm{vsc}\right.$ and esc) such that at the initial point $x=x_{0}$ the scaled values ( $\left.x_{i} / x s c_{i}, v / v s c, e / e s c\right)$ give $W$ and its first and second derivatives approximately unit mamitude (Haarhoff and Buys (65)). In this case $K$ may be chosen relatively small and often kept constant throughout the minimization ( $K=20$ is suitable).

(d) Validification of Tangency Point

For convergence let $\underline{x}=\underline{\underline{x}}$ be the minimizing value of the PF for the last cycle. Since $\dot{V}(\underline{\underline{x}}) \times 0_{\text {may not be satisfied }}$ x is repeatedly multiplied by a scalar $\gamma$, close to one, until $\dot{v}\left(\gamma^{j} \underline{\underline{x}}\right)<0$ for some $j\left(e . g \gamma=1-10^{-4}\right)$. This gives $\underline{x}_{m}=\gamma^{j} \underline{x}_{\text {as }}$ a modified tangency point and a possible stability boundary of

$$
\underline{x}^{\top} \underline{x}=\underline{x}_{m}^{\top} \underline{P}_{m}=v_{m} \quad 5.4 .7
$$

The validity of the latter is tested by evaluating $\dot{V}_{M}(L)$ in 5.2 .15 with a fine mesh. If $\dot{V}_{M}(L)>0$ the maximizing vector giving $\dot{V}_{M}(L)$ is found, $z$ say, and the interval $(0,1.0)$ is successively halved until bracketing numbers $\mathrm{E}_{1}$ and $\mathrm{E}_{2}$ are found satisfying $O<\mathrm{E}_{1}<\mathrm{E}_{2}<1.0$ such that

$$
\left|\frac{V\left(E_{2} z\right)-V\left(E_{1} z\right)}{V\left(E_{2} z\right)}\right|<.1
$$

where $\bar{V}\left(E_{1} z\right)<0$ and $\dot{V}\left(E_{2} z\right)>0$. The vector $\underline{x}_{0}=E_{1} z$ determines a new starting point for the penalty function and the minimization is repeated. 
Since $V m$ in 5.4.7 decreases for successive tangency points obtained, the method converges to a valid RAS boundary. However, if it is suspected that two tangency points exist giving almost the same boundary in 5.4 .7 , then either both of these must be found or an extremely fine mesh is required to evaluate $\dot{\mathrm{V}}_{M}(\mathrm{~L})$.

\section{Comment}

A slightly quicker, but less useful, initial point $x_{0}$ in (b) may be obtained by expanding the sphere $x^{T} x=1$ instead of the boundary $\underline{x}^{T} P_{\underline{x}}=1$.

The choice of $\mathrm{PF}$ in 5.4 .4 is not arbitrary. Infact that of Miele was compared to one of Fletcher and Lill (63). They use a PF which has a stationary point at a solution of the constrained problem thus avoiding a sequential approach. With suitable q they minimize

$$
w=v-e\left[\frac{\underline{a}^{T} \underline{b}}{\underline{a}^{\mathrm{a}}}\right]+\frac{q}{2} \frac{e^{2}}{\left(\underline{a}^{\mathrm{T}} \underline{a}\right)}
$$

with $\underline{a}=\nabla e$ and $\underline{b}=\nabla V$. The second term is here a continuous approximation to the Lagrange multiplier. However, with limited experience the method was not as good as Miele's.

Fig 5.6 gives a general flow diagram for the algorithr which was programmed in Fortran IV. Table 5.2 shows some typical features when applied to the system S1, for a simple quadratic, and 511 for the best quadratic of chapter 4 , the latter showing the effect of two tangency points. Computation time seems large but this is due to the chosen values of $N_{n}, N_{2}=31$ and $N_{3}=160$, for the initial boundary. $\left(\mathrm{N}_{2}=10\right.$ and $\mathrm{N}_{3}=50$ would suffice, although giving a worse starting point $\left.x_{0}\right)$. 
TABLE 5.2

\begin{tabular}{|c|c|c|}
\hline SYSTEM & ST & $\mathrm{s} 11$ \\
\hline $\mathbf{p}$ & I & $\begin{array}{rrr}18.101 & -15.853 & 1.878 \\
. & 31.936 & -16.586 \\
. & . & 17.194\end{array}$ \\
\hline $\begin{array}{l}\text { EVALUATIONS OF } \\
\dot{\mathrm{V}}_{\mathrm{M}}(1)\end{array}$ & 8 & 8 \\
\hline FINAL 1 & 1.2 & 0.375 \\
\hline$N_{n}$ & 31 & 160 \\
\hline $\begin{array}{c}\text { CONVERGENCE VALUE : } \\
(5.4 .5)\end{array}$ & $\epsilon=10^{-4}$ & $\epsilon=10^{-4}$ \\
\hline \multirow{2}{*}{$\begin{array}{l}\text { SCALE FACTORS } \\
\left(x S C_{i}, \text { VSC, esC) }\right.\end{array}$} & \multirow{2}{*}{ NONE } & $05, .05, .053,4.996,1.4 \times 10$ \\
\hline & & $0.11, .05, .05,4.33, .029$ \\
\hline INITIAL $\mathbf{x}_{0}$ & $1.05,0.575$ & $\begin{array}{l}.229, .287, .0745 \\
-.420,-.384, .116 \\
\end{array}$ \\
\hline INITIAL $\mathrm{v}, \lambda, \mathrm{K}$ & $1.44,0.0,1.0$ & $\begin{array}{l}4.996,0.0,20.0 \\
4.33,0.0,20.0\end{array}$ \\
\hline NO. OF CYCLES & 3 & $\begin{array}{rr}5 \\
\end{array} \quad 10 \quad 3$. \\
\hline FINAL $\underline{x}$ (TAN.PT. ) & $1.0746,0.6204$ & $\begin{array}{l}.22101, .2937, .1447 \text { (INVAI } \\
+=.36841,-.2614, . .2496 \text { (VAI }\end{array}$ \\
\hline FINAL $\mathrm{V}, \lambda, \mathrm{K}$ & $\begin{array}{r}1.5365,-.769 \\
1.0\end{array}$ & $\begin{array}{l}4.630,-.36961,20.0 \\
4.467,-1.156,20.0\end{array}$ \\
\hline FINAL VOL & $\begin{array}{r}4.8271 \\
\quad(\mathrm{AREA}) \\
\end{array}$ & 0.763 \\
\hline TIME (MILL/SECS.) & 15 & 207 \\
\hline
\end{tabular}

* SECOND MINIMIZATION 
Master Segment for High Order OPTIMAL QUADRATIC ALGORITHM

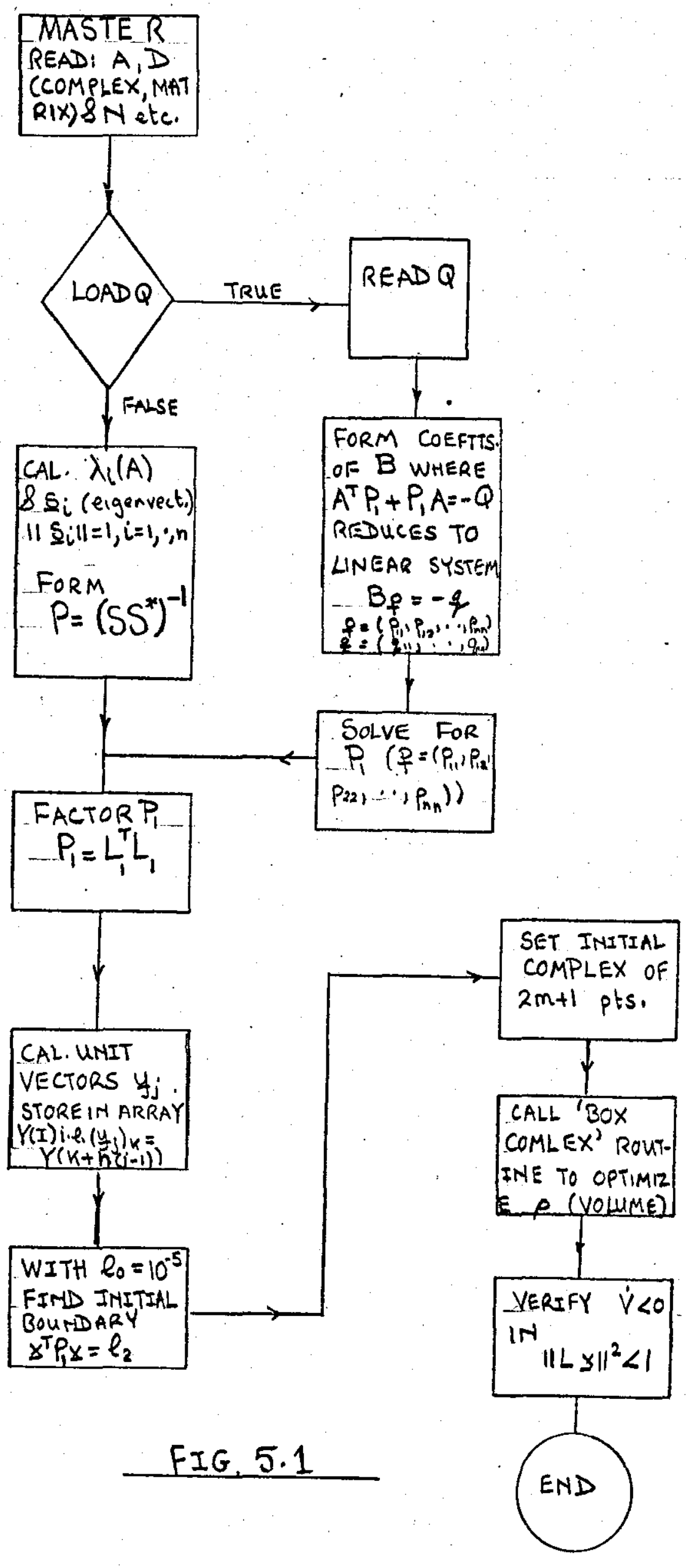


SUBROUTINE DIVISION OF HIGH ORDER OPTIMAL QUADRATIC ALGORITHM

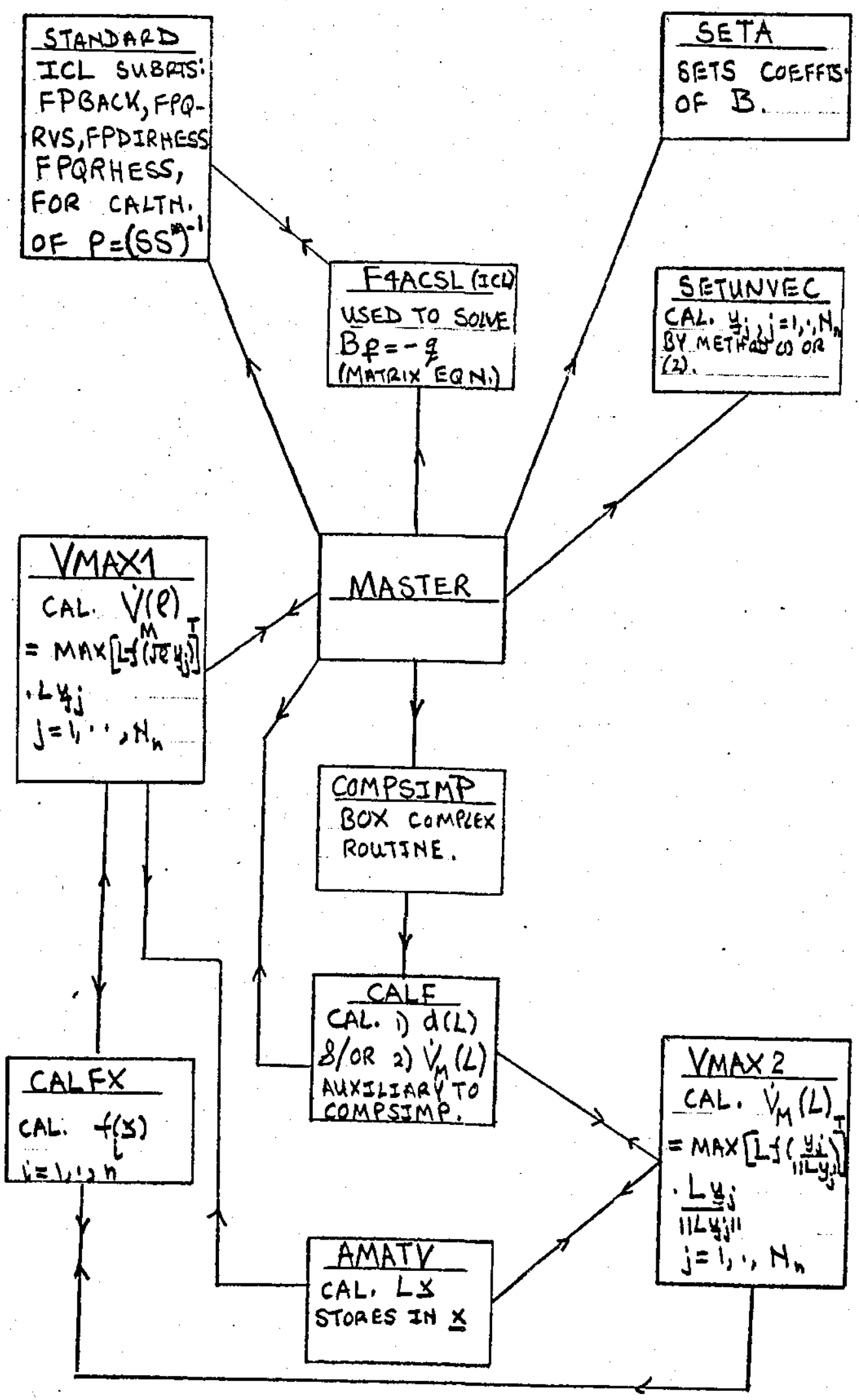

FIG. 5.2 


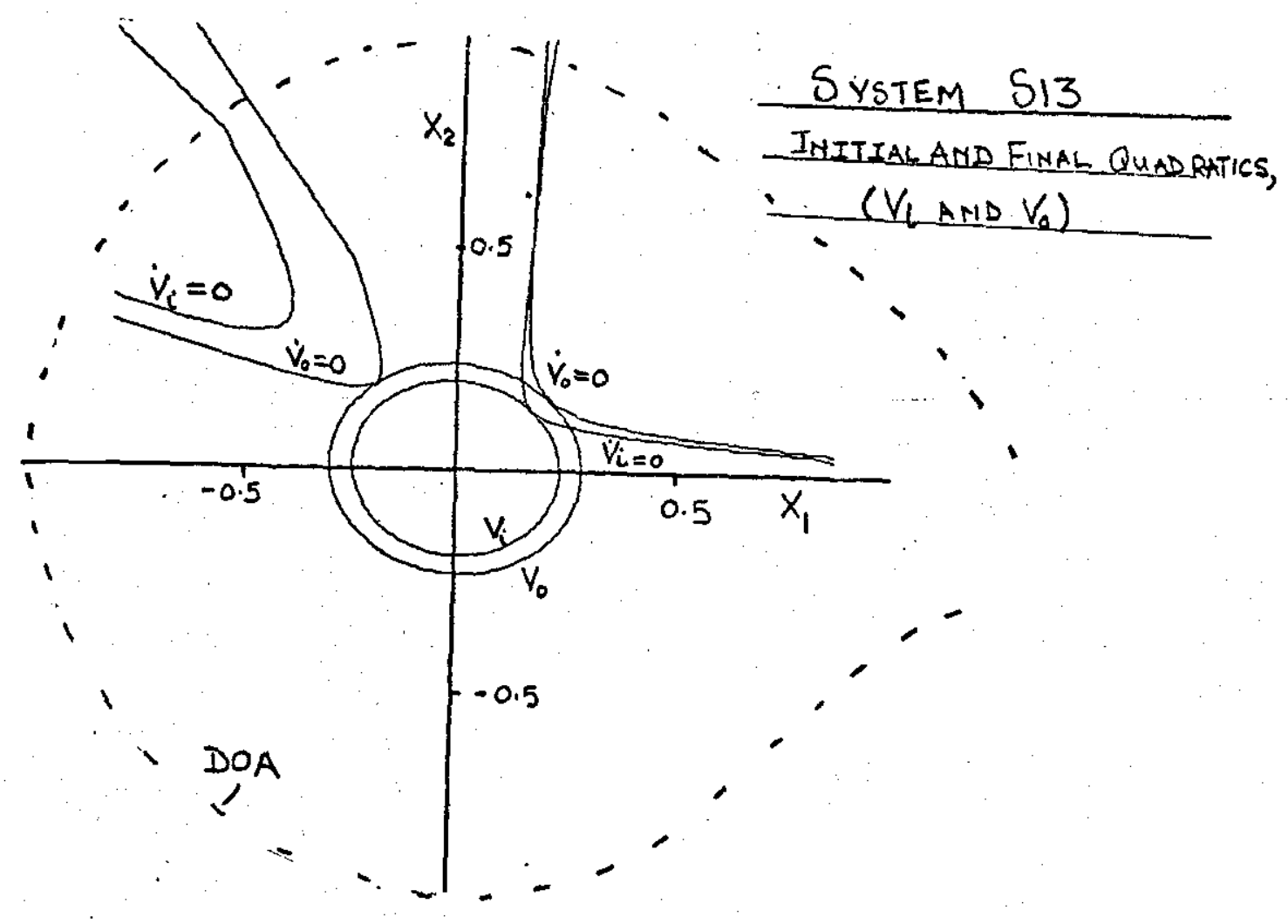

FIG. 5.3

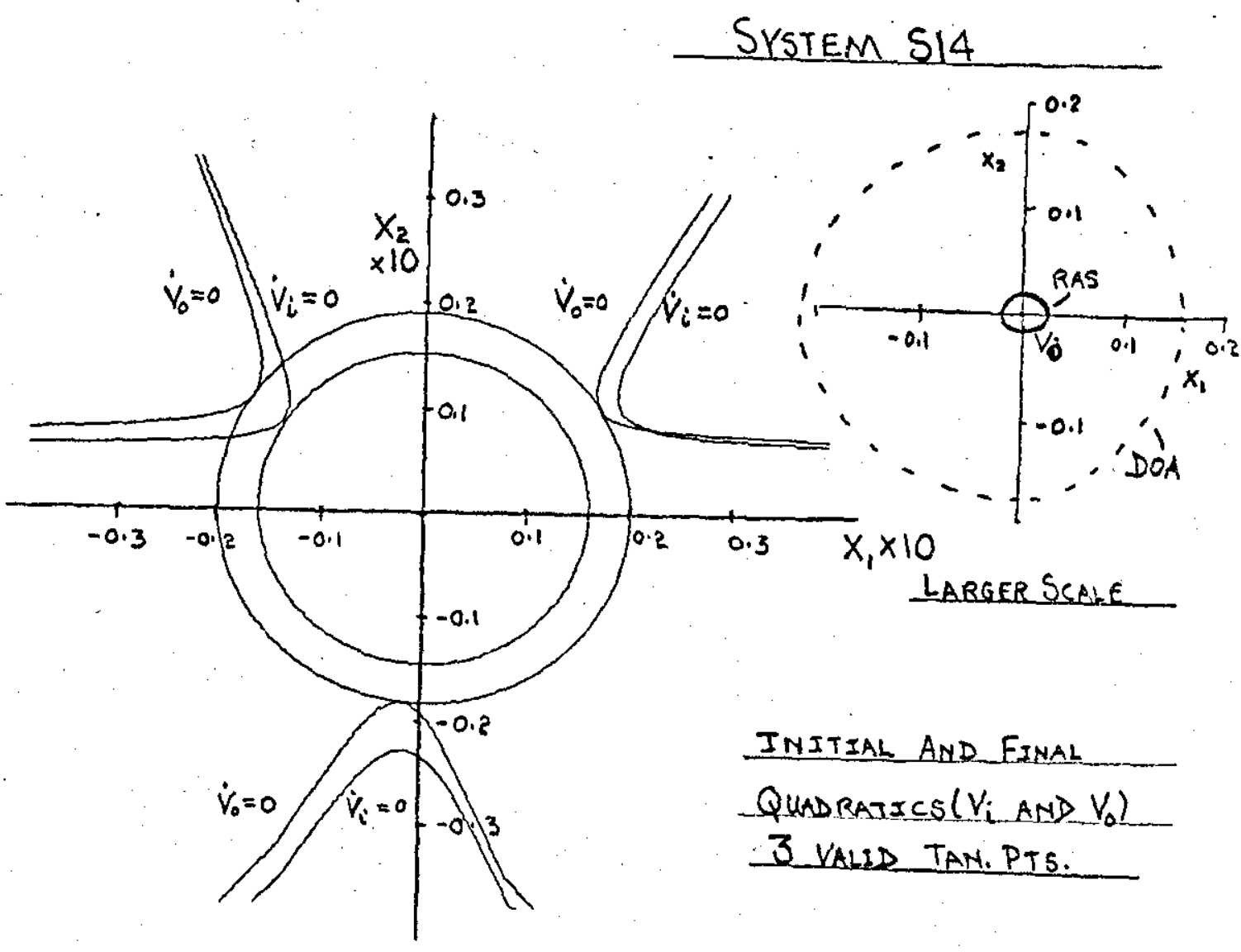

FIG. 5.4 

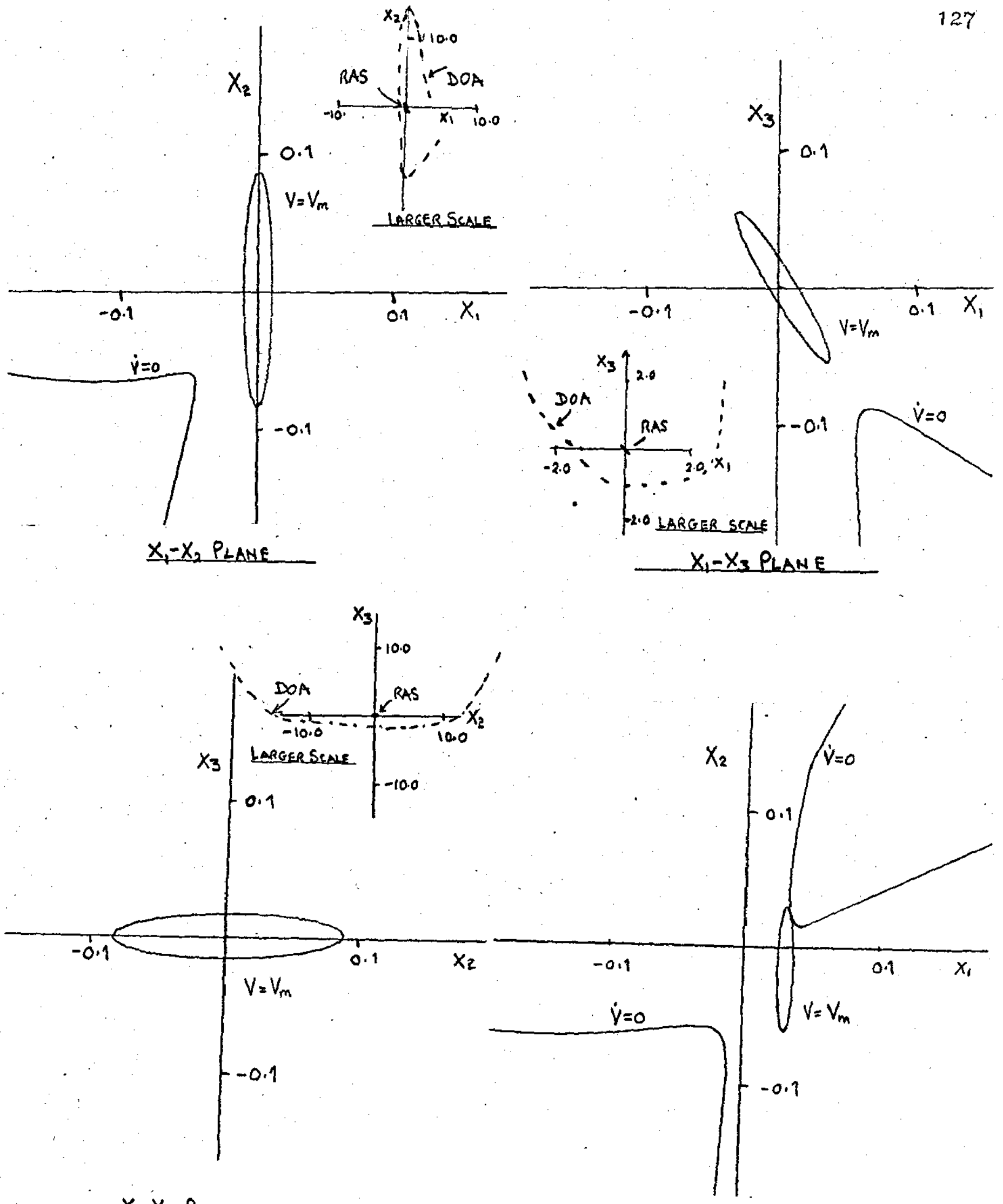

$x_{2}-x_{3}$ Pare

SECTION IHRO $X_{3}=-.0483 \| t_{0} X_{1}-X_{2}$ PLANE

SYSTEM S17 (RAO(5))

FIG. 5.5 
Flow Diag. For QUadRatic RAS Determination

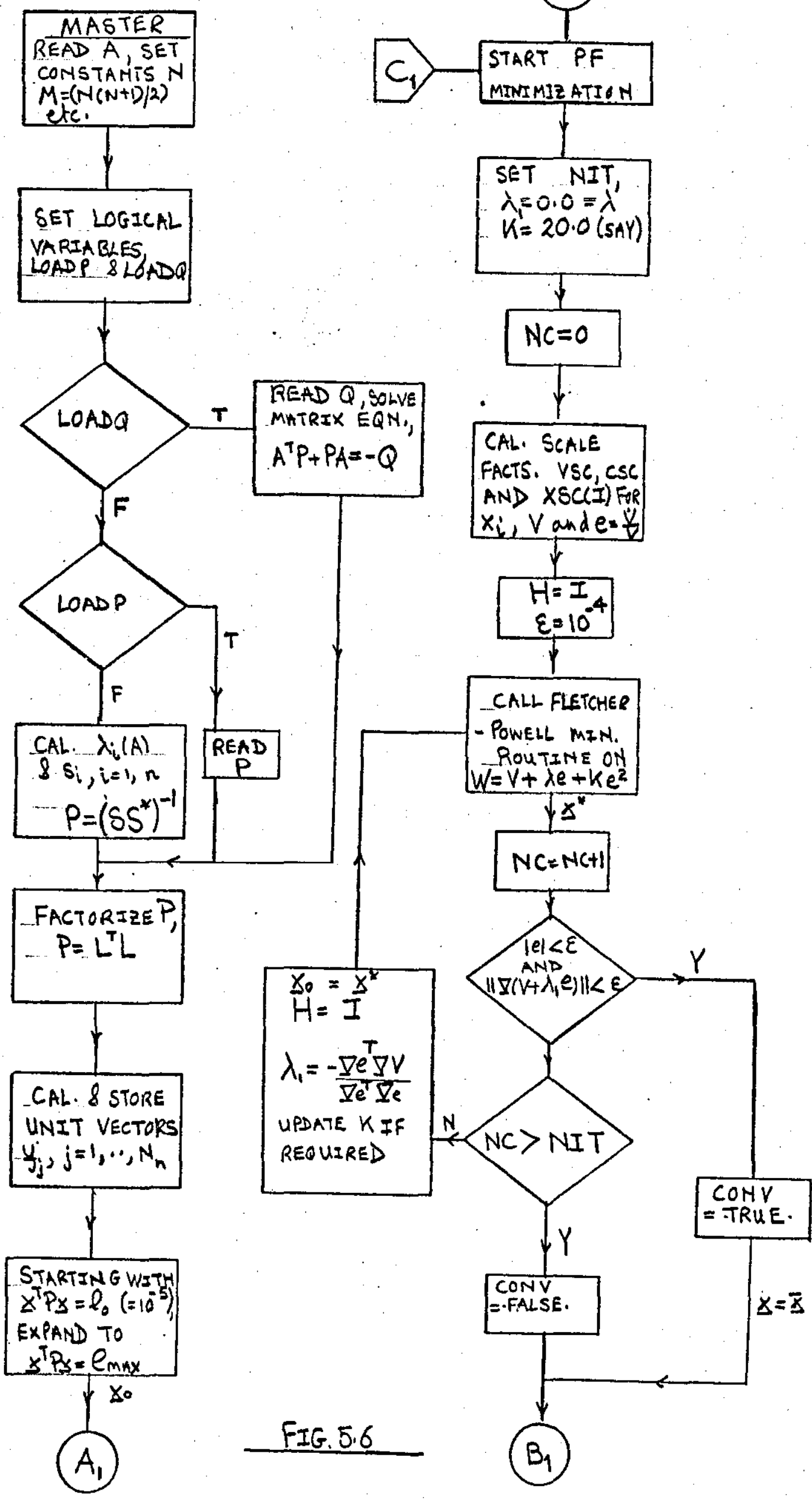




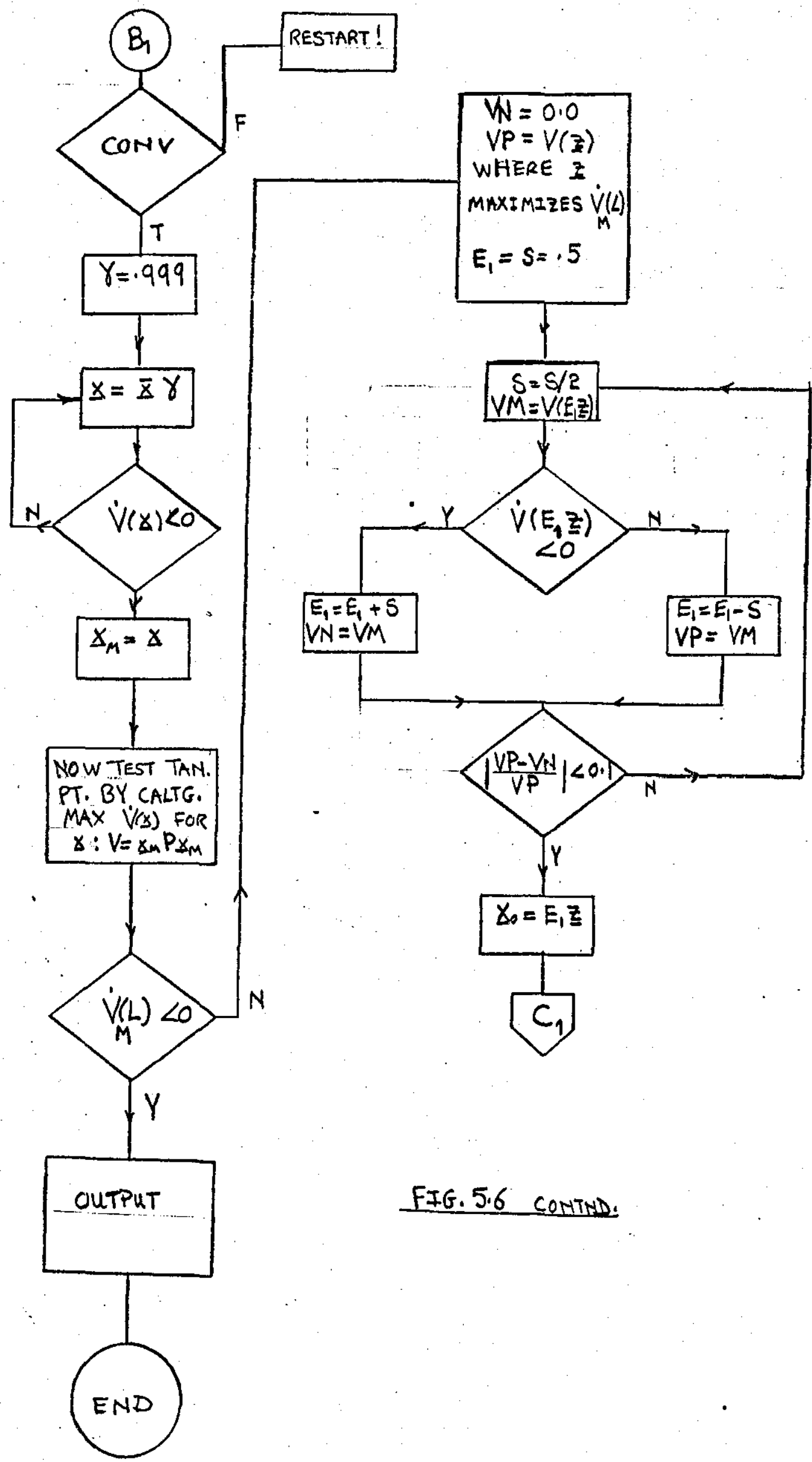


CHAPTER 6

GENERAL OPTIMAL LYAPUNOV FUNCTIONS

FOR NON-LINEAR SYSTEHS INCUUDING

THOSE OF LURE' FORM AND RELAY

CONTROL SYSTEMS. 


\section{Chapter 6}

\section{1 High Degree Lyamunov Functions for Autonomus Non-Linear} Systems.

\section{Introduction}

A natural extension of a quadratic LF for the stable system of form 4.1.1 in Chapter 4, namely,

$$
\underline{x}=A \underline{x}+g(\underline{x})
$$

is the LF of degree $\mathrm{mv}$,

$$
v=v_{2}+v_{3}+\cdots+v_{m v}
$$

$$
6.1 .2
$$

where $v_{i}$ is a homogeneous polynomial of degree $i$ and $v_{2}$, $v_{2}=\underline{x}^{T} P_{x}$, is itself a LF for the system. For second order systems we write

$$
v=\sum_{i=2}^{m v} \sum_{j=1}^{i+1} v_{i j} x_{1}^{i-j+1} x_{2}^{j-1} \quad 6.1 \cdot 3
$$

which involves to a multiplicative constant $\mathrm{m}=2+(\mathrm{mv}+5)(\mathrm{mv}-2) / 2$ parameters, $\mathrm{v}_{i j}$, which is large for $m v \geq 5(m \geq 17)$.

A less obvious class is the product of quadratics.

$$
v_{2 m v}=\prod_{k=1}^{m v} \underline{x}^{T} P_{k} \underline{x}
$$

where the $p_{1}$ are p.d.s. matrices which solve

$$
A^{T} P_{k}+P_{k} A=-Q_{k} \quad(k=1, \cdots, \text { mv })
$$

for p.d.s. $Q_{k}$. This LF has the simplicity of the quadratic and only $\mathrm{m}=2 \mathrm{mv}$ parameters need determining.

- Two questions are apparent:

a) How do the optimal RAS'S for high degree LF's compare with those of the quadratic? 
b) Are any 'equal tangency phenomena' present such as property A for optimal quadratics?

In order to give answers one must resort to numerical means as the RAS problem is intractable analytically. Henceforth we discuss some of the numerical features in determining the optimal LF's.

\section{RAS Determination and Optimization Methods}

The RAS problem in 4.1.10 and 4.1.11 of minimizing $V$ on the constraint $\dot{V}=0$ is more involved for a general LF of the form 6.1 .3 and can cause difficulty with numerical methods. The latter can be attributed to the fact that (a) $\nabla V$ may vanish at a relative minimum and contact between the boundary $\mathrm{V}=\mathrm{V}_{\mathrm{m}}$ and the constraint contour is not smooth, (b) the contours of $\mathrm{V}$ and $\dot{\mathrm{V}}=0$ may have several components and (c) $V$ may not be p.d.in $\mathrm{s}^{\mathrm{n}}$. (Note (a), (b) and (c) are not unrelated and also that points satisfying $\underline{\nabla V}(\underline{x})=0$ give tangency points)

Given the LF 6.1 .3 a RAS was obtained for a second order system via Hewit's (2) version of Rodden's method (3). In essence the main features are:

(1) a search for the constraint contour by spiralling out from the origin until a point $x_{1}$ is reached s.t. $\dot{v}\left(\underline{x}_{1}\right)>0$, followed by an accurate location of the constraint at $\underline{x}_{2}$ say.

(2) A step is taken along a vector tangential to $\dot{V}=0$ at $x_{2}$ in a direction of decreasing $V$ followed by a search along $\vec{\nabla} \dot{V}$ to relocate the constraint. This is repeated until tangency, and a possible stability boundary is found.

(3) Steps along this tentative stability boundary, $V=c$, are made and $\dot{V}(\underline{x})$ is evaluated at each step. If $\dot{v}(\underline{x})>0$ the 
previous tangency point is invalid, and having relocated the constraint, step (2) is repeated. Unless the method breaks down a conservative RAS is obtained of $V=c<V_{m}$ and its average radius is found.

To determine the optimal LF of 6.1 .3 with respect to the numerical average radius $p$, the Nelder and Mead (46) simplex method was used. The optimization was over the $m$ parameters

$$
\begin{aligned}
t_{1}, t_{2} \text { and } v_{i j}, i & =3, \ldots, \mathrm{mv} \\
j & =1, \ldots, i+1
\end{aligned}
$$

where $t_{1}$ and $t_{2}$ determine $Q$ as $Q=L^{T} L+\epsilon I$, and $P$ solves the matrix equation 4.1.5, the latter ensuring 6.1 .3 is a LF for 6.1.1. In the numerical results which follow Rodden's method continually broke down for higher degree LF's due to difficulties (a) to (c) mentioned above and necessary features were incorporated:

a) an upper bound on points, $\underline{x}_{i}$ say, which search the constraint contour in step (2), $\left\|x_{i}\right\|>R_{\max }$,

b) a test for $v\left(\underline{x}_{i}\right)>v_{\max }$ at tangency,

c) a test for a pt. $\underline{x}_{2}$ lying on $v=c$ (stability boundary), of $\left\|x_{2}\right\|>R_{\max }$ (i.e a test for an open boundary),

d) a test to discover whether step (2) of Rodden's method was repeatedly converging to the same tangency point. If any of the bounds in (a) to (c) vere satisfied the RAS function value of $p$ for Nelder \& Mead was penalized (i.e $p=$ average radius $=-10$ say). In case (d) the best tangency point out of three was found.

The LF in 6.1 .4 was investigated for the restricted class of systems of $\mathrm{C} 4$ such that, with minor modifications, the 2nd order optimal quadratic algorithm could be used. The optimization of $\rho$, the average radius, was over the $2 \mathrm{mv}$ 
values

$$
t_{k 1}, \dot{t}_{k 2} \quad ; \quad l k=1, \mathrm{mv}
$$

where $Q_{k}=L_{k} T_{k}+\epsilon I>0$ and

$$
L_{1 k}=\left[\begin{array}{ll}
1 & t_{k 1} \\
0 & t_{k 2}
\end{array}\right]
$$

As for the quadratic, a boundary $V=c$ is easily traced out for if $y$ is any vector then $V(a y)=c$ where

$$
a=\left(c / \prod_{k=1}^{m y} \prod_{k} y\right)^{\frac{1}{2 \operatorname{lnv}}}
$$

This fact also means that the complex algorithm of $\mathrm{C}_{5}$ can'be extended to this IF to study high order systems, the average radius being easily obtained from the evaluation of $\dot{V}_{M}\left(L_{k}\right)$. in 5.2 .15 .

Numerical Examples of Optimal LF's for $\mathrm{V}_{\mathrm{mv}}=\prod_{\mathrm{k}=1}^{\mathrm{mv}} \underline{x}_{\mathrm{k}}^{\mathrm{T}}$

In the examples that follow the average number of function evaluations of $\rho$ by Povell's conjugate direction. algorithm for the LF's of degree 2,4 and 6 were respectively 70,140 and 220, giving an accuracy of $10^{-2}$ or more for the optimum $\rho$ (average radius). The latter was calculated from 80 points on the boundary $\mathrm{V}=\mathrm{V}_{\mathrm{m}}$.

Table 6.1 shows a comparison of $\rho$ for 4 systems taken from $\mathrm{C}_{4}$. Some marked improvement exists for systems $\mathrm{s} 1, \mathrm{~S} 2$ and 57 where the DOA's are radially symmetric, but for $s 6$ the difference between the optimal $\rho$ for $v_{2}$ and $v_{6}$ is poor.

It was important in the optimization of $\rho$ for $v_{4}$ and $v_{6}$ not to choose all initial $L_{k}(k=1,3)$ equal $\left(L_{k}=I\right.$ say or $L_{k}=L_{0}$ where $L_{0}$ gives the optimal quadratic.) This 
latter choice usually corresponded to a local minimum where the Powell optimization tended to stick due to the two valid tan. pts. of the $O Q$ (Note the RAS's of $\mathrm{V}^{\mathrm{m}}(\underline{\mathrm{x}}$ ) and $V(x)$ are the same).

Fig. 6.1 shows the relationship between the optimal boundaries of $\mathrm{V}_{2}, \mathrm{~V}_{4}$ and $\mathrm{V}_{6}$ and their constraint contours for system S1. For the $4^{\text {th }}$ degree $L F$ there are 2 valid $R S$ tangency points and 3 RS constraint components, whereas for the $6^{\text {th }}$ degree the numbers are 2 and 4 respectively. As for the quadratic case, these optimal LF's vere non-unique. From numerical evidence it seems highly probable that at the exact optima for $V_{4}$ and $V_{6}$ there are 3 and 4 RS valid tangency points respectively. Interestingly, the non-convex shapes of the higher degree boundaries resemble those of the DOA more clearly.

Similar considerations apply to the corresponding boundaries for system S7, shown in Fig. 6.2, with an equal tangency property holding for $v_{4}$ and $v_{6}$ with two $R S$ valid tangency points and 3 and 5 RS constraint components respectively. (There is almost a third point of contact of $\mathrm{v}_{6}=\mathrm{v}_{\mathrm{m}}$ with $\dot{\mathrm{V}}_{6}=0$ ). All the optimal boundaries pass through the singular point $(1,2)$ of system $\mathrm{S7}$. 
TABLE 6.1

\begin{tabular}{|c|c|c|c|}
\hline \multirow{2}{*}{ SYSTEN } & $\mathrm{m}=2$ & $\mathrm{~m}=4$ & $\mathrm{~m}=6$ \\
\hline S1 & $\mathrm{v}_{2}$ & $\mathrm{v}_{4}$ & $\mathrm{v}_{6}$ \\
\hline (ZUBOV) & 1.883 & 2.219 & 2.292 \\
\hline S2 & & & \\
\hline VAN.POL) & 1.44 & 1.51 & 1.57 \\
\hline S6 & & & \\
\hline (DAVIES $)$ & 0.1857 & 0.1873 & 0.1874 \\
\hline S7 & & & 3.21 \\
\hline
\end{tabular}

Numerical Ixamples of Optimal LF's Fox $V$ in Series Form $(6.1 .3)$

An obvious initial choice for the coefficients $v_{i j}$ in 6.1 .3 for $\mathrm{mv}>2$ is

a) for $V_{m v}$ select as initial values for

$$
\begin{aligned}
v_{i j}, \quad j & =2, \ldots, \quad m v-1 \\
j & =1, \ldots, i+1
\end{aligned}
$$

those of the optimal. LF of degree $\mathrm{mv}-1$ and

b) put $v_{m v j}=0.0, j=1, \ldots, m v+1$

or c) put $v_{\mathrm{mvj}} \neq 0.0$, " "

The choice of a) and b) was unsatisfactory since the optimization started on an equal tancency surface and convergence was poor. Although the other choice is better a) and c), arbitrarily selecting all coefficients of the same magnitude proved as good. 
For convenience we write the LF in 6.1 .3 as $V_{2}, 3, \ldots$, nv' then Figures 6.3 to 6.6 show the behaviour of the optimal LF's with respect to the following systens:

\section{System S2 (Van dor PoI)}

Since the DOA for the system is radially symmetric it was reasonable to choose the $L F$ accordingly and so the forms $\mathrm{v}_{2}, \mathrm{v}_{2,4}$ and $\mathrm{v}_{2,4,6}$ vere investigated for which $m=2,7$ and 14 respectively. The simplex optinization was terminated when either the difference between function values of the simplex were less than $10^{-3}$ in magnitude or when $I$, the number of function evaluations exceeded an upper bound $I_{\text {MAX, }}$ the latter beine 50,190 and 290 for the respective cases.

For the IF $\mathrm{V}_{2,4}$ convergence was obtained in 184 function evaluations (FE's) but not without many of the breakdowns of Rodden ((a) to (d)), this being the case for the following systems as we11. Valid tangency alternated between 3 distinct RS points near the optimum and is reflected in Fig. 6.3 where the optimal boundary has 3 RS valid tangency points in contact with its constraint contour, the latter having 2 RS components which are unlike those of the quadratic.

For the LF $V_{2,4,6}$ convergence was very poor in that $\rho$ tended to stick to a value near the optimum, which was in part due to the high dimension of the space of parameters $a_{i j}(m=14)$ for which the Simplex method becomes inefficient. However, after several restarts an accurate optimum was obtained and Fig. 6.3 shows that the optimal boundary, on which 3 valid RS tangency points lie, gives a good RAS 
both in shape and size. The corresponding table shows the large computing time for $\mathrm{v}_{2,4,6^{\circ}}$

\section{System $\$ 18$}

Consider the system

$$
\begin{aligned}
& \dot{x}_{1}=x_{2} \\
& \dot{x}_{2}=-x_{1}\left(1-x_{1}^{2}\right)-x_{2}\left(1-x_{2}^{2}\right)
\end{aligned}
$$

with singular points $(0,0)$ and $\mp(1,0)$.

Investigating the same LF's shows the situation is somewhat different, since for all LF's, only one RS constraint component exists near the origin and the respective optimal boundaries all have two RS points of contact with the latter (Fig. 6.4). As for the previous system the average radius of the $6^{\text {th }}$ degree LF is only fractionally better than that of the $4^{\text {th }}$ and the RAS's are poor compared to the DOA.

\section{System 519}

A system with a non-symmetric DOA is.

$$
\begin{aligned}
& \dot{x}_{1}=x_{2} \\
& \dot{x}_{2}=-x_{1}\left(1+x_{2}\right)-x_{2}\left(1-x_{2}^{2}\right)
\end{aligned}
$$

In this case the LF's $V_{2}, V_{2,3}$ and $V_{2,3,4}$ were investigated. Fig. 6.5 shows the optimal boundaries of the $3^{\text {rd }}$ and $4^{\text {th }}$ degree curves having 3 points of contact with their constraint contours (the non-optinal ones having one or two). Their respective RAS's have the usual failing for systems with open DOA's, being very poor. 


\section{System S13 (Yu (6))}

The system is that in 5.3 and caused some difficulty in convergence of the average radius to the optimum for $\mathrm{V}_{2,3,4}$. This is shown in Fir. 6.6 where al though the optimal boundary for $v_{2,3}$ has almost 3 points of contact with its constraint contour, that of $v_{2,3,4}$ has one. Also, due to continued breakdown of the Rodden method (mainly tangency points giving open boundaries), the latter required 6000 mill/sec for $59 \mathrm{FE}$ 's after restarting near the optimum. However, some marked improvement is shown in the RAS.

\section{Comment}

It is evident that higli degree optimal LF's do in some cases give much improved RAS's (systems s1, s7, s2, S19), but the equal tangency phenomenon which is also present and contributes to lack of convergence, thereby making computing time excessive, offsets this advantage.

All systems studied had two or more valid tangency points for the optimal LF's of degree greater than two. The direct relationship between the number of tangency points and valid tangency points is complicated by the multiple components of both the $\mathrm{v}_{\mathrm{mv}}=$ const. and $\dot{\mathrm{v}}_{\mathrm{mv}}=0$ contours. Depending on the system, the number of tanfency points, valid tangency points and constraint components tend to increase with mv. 


\subsection{Optimum Lyapunov Junctions For Relay Control Systems}

\section{Introduction}

The application of Lyapunov's direct method to differential equations with discontinuous right hand sides has been studied in particular by Alimov (69), Ansonov (68) and Veissenberger $(48,66)$. In this section ve shall extend some work of the latter and consider the relay system

$$
\dot{x}=A \underline{x}+\underline{b} \sin \sigma, \quad \sigma=\underline{a}^{T} \underline{x} \quad 6.2 .1
$$

where

$$
\operatorname{sgn} \sigma=\left\{\begin{array}{l}
+1, \sigma>0 \\
\epsilon, \sigma=0, \quad-1 \leq \epsilon \leq 1 \\
-1, \sigma<0
\end{array}\right.
$$

We assume, as for most practical systems, that

$$
\underline{d}^{\mathrm{T}} \underline{\mathrm{b}}<0 \quad 6.2 .3
$$

which implies two types of motion: repular syitching in which trajectories in $\sigma>0$ connect on $\sigma=0$ with trajectories in $\sigma<0$, and sliding, in which segments of the trajectories lie in the space $\sigma(\underline{x})=0$. A complete discussion of these motions is given in reference (48) and we mention that the main definitions and theorems - including Theorem 1.5.3 - of Iyapunov's direct method are applicable to relay systems provided the stability of the two motions are treated separately.

For regular switching the motion is described by 6.2.1, while for sliding, which occurs for $\left|\underline{d}^{T} A x\right|<\left|\underline{d}^{T} \underline{b}\right|$ on $\underline{d}^{T} x=0$, by the linear system

$$
\dot{x}=\bar{x} \underline{x}
$$


where

$$
\vec{A}=\left(I-\frac{\underline{b \underline{d}^{T}}}{\underline{d}^{\mathrm{T}}}\right) A
$$

By considering an orthogonal transformation from $x$ to $y$ via

$$
y=G \underline{x} \quad\left(G^{T}=G^{-1}\right) \quad 6.2 .6
$$

such that $y_{n}$ is normal to the sliding plane, $\sigma=0$ i.e

$$
(0,0, \ldots, 0,1)=\mathrm{Gd} /\|\underline{\mathrm{d}}\|
$$

the system 6.2 .4 may be written

$$
\dot{\mathrm{y}}=\mathrm{G} \overline{\mathrm{A}} \mathrm{G}^{\mathrm{T}} \mathrm{y} \quad 6.2 .7
$$

Further, since $\dot{y}_{n}=0$, by defining a new vector $\underline{z}$ as $\underline{z}=\left(y_{1}, y_{2}, \ldots, y_{n-1}\right)$, we obtain

$$
\underline{\mathbf{z}}=\mathrm{A}^{\prime} \underline{\mathrm{z}}
$$$$
6.2 .8
$$

where $A^{\prime}$ is the $(n-1) x(n-1)$ matrix obtained by deleting the last row and column of $\mathrm{G}_{\bar{A}}{ }^{\mathrm{T}}$.

Two candidates for a IF for 6.2 .1 are

a) the piecerise quadratic LF

$$
\begin{aligned}
V & =\underline{x}^{T} P \underline{x}+\int_{0}^{\sigma} \operatorname{sgn} s d s \\
& =\underline{x}^{T} \underline{x}+\left|\underline{d}^{T} \underline{x}\right|
\end{aligned}
$$

for which $\dot{V}=\underline{x}^{T} \underline{x}+\underline{K}^{T} \underline{x} \operatorname{sgn} \sigma+\underline{d}^{T} \underline{b} \quad 6.2 .10$

where $\quad Q=A^{T} P+P A$

and $\quad \underline{K}=A^{T} \underline{d}+2 P \underline{b}$

(By $6.2 .3 \mathrm{~V}>0$ and $\dot{\mathrm{V}}<0$ for some $R(\mathrm{~h})$, h small, $\sigma \neq 0$ ). Here Weissenbexcer has shown that $V$ is a LF for 6.2 .1 iff the symetric $P$ has the property 


$$
A^{\prime} P^{\prime}+P^{\prime} A^{\prime}=-Q^{\prime}<O^{\prime} \quad 6.2 .11
$$

where $A^{\prime}$ is from 6.2 .8 and $P^{\prime}$ is formed by deleting the last row and column of $\mathrm{GPG}^{\mathrm{T}}$. Here, $\mathrm{V}$ may still be a LF even though $P$ is indefinite and/or $A$ is unstable.

b) the piecevise linear IF

$$
v=\sum_{i=1}^{m}\left|c_{i}^{\mathrm{T}} \mathbf{2}\right| 6.2 .12
$$

originally used by Rosenbrock (70), where for $6.2 .1 \underline{c}_{i}=\underline{d}$ for some $i$ and, to ensure $y$ is p.d. the vectors $\varepsilon_{j}$ span $E^{n}$. The contours of $\mathrm{V}=$ constant are composed of at most $2^{\mathrm{m}}$ hyperplanes whose normals satisfy

$$
\underline{n}_{j}=\sum_{i=1}^{m} \bar{t}_{i}, \quad j=2, \ldots, 2^{\mathrm{m}}
$$

Then since 6.2 .1 is linear for $\sigma>0$, by Rosenbrock's analysis, if

$$
\underline{x}^{\mathrm{T}} \underline{\mathrm{n}}_{i} \leqslant 0
$$$$
6.2 .13
$$

at each vertex of the face whose normal is $\underline{n}_{i}$, for all faces of $\mathrm{V}=\mathrm{c}$, then $\mathrm{V}<\mathrm{c}$ is an estimate of the RAS provided 6.2 .13 also holds for sliding.

\section{The Piecewise Quadratic LF}

An RAS determination for the LF 6.2 .9 is essentially the same as in 6.1 of minimizing $V$ on the constraint contour $\dot{V}=0$ from 6.2 .10 , where in the plane $\sigma=0$ the latter is discontinuous and given by

$$
\dot{v}=\underline{x} \underline{\underline{x}}+\underline{K x}+\underline{d}^{T} \underline{b}=0
$$

Weissenberger (48) has given a second order example where he optimizes the area of the RAS for LF 6.2.9. He produces 
an optinal boundary having 2 RS points of contact with its constraint, but goes no further. Here we explore the situation more fully.

Since points on $\dot{V}=0$ are easily determined by solving a quadratic, the second order algorithr of chapter 4 is again applicable. The major modification in RAS deternination is the replacement of the one-dimensional search routine using, continuity for quadratic fits, by the method of search by Golden Section (56). This is due to the two types of tansency which occur:

a) a smooth tangency at which $V$ and $\dot{V}=0$ are continuous and

b) a corner tangency lying on $\sigma=0$ where the contours of $\dot{\mathrm{V}}=0$ may 'jump' discontinuously.

The optimization of the area of the RAS was made over the space of the elements of $p\left(p_{11}, p_{12}, p_{22}\right)$ subject to 6.2 .11

\section{Numerical Examples}

\section{System 520}

A relay system having a limit cycle is

$$
\begin{aligned}
& \dot{x}_{1}=x_{2} \\
& \dot{x}_{2}=-x_{1}+x_{2}-\operatorname{sgn}\left(x_{1}+x_{2}\right)
\end{aligned}
$$

Optimization of the RAS area by Powell's method showed a marked oscillatory effect of the valid tangency point between a corner and a smooth tangency. Fig. 6.7 shows an initial LT boundary

$$
2 x_{1}^{2}-x_{1} x_{2}+x_{2}^{2}+\left|x_{1}+x_{2}\right|=1.705
$$

(with $\rho=2.094$ ) with one strooth $R$ S valid tangency and the best LF boundary 


$$
1.589 x_{1}^{2}-x_{1} x_{2}+x_{2}^{2}+\left|x_{1}+x_{2}\right|=1.953
$$

with a corner and a smooth RS valid tangency point, $(\rho=2.73)$. In this case the constraint contours are closed. That one equal tangency surface exists, similar to the curve in the $O Q$ case (C4), is seen $i n F i g .6 .8 \mathrm{a}$ and $6.8 \mathrm{~b}$ where the actual area contours have been plotted through sections of the $3 \mathrm{dim}$. space of elements of $P$ paralle1 to the $\mathrm{p}_{11}-\mathrm{p}_{22}$ plane for $\mathrm{p}_{12}=0.0$ and $\mathrm{p}_{12}=-.5$ respectively (Shown $A B$ ). Values of $P$ giving smooth tangency lie above surface $A B$, the optimal $P$ lying on the section of $A B$ in $F i g .6 .8 b$.

\section{System S21}

An exanple showing a marked discontinuity of $\dot{V}$ on $\sigma=0$ is

$$
\begin{aligned}
& \dot{x}_{1}=x_{2} \\
& \dot{x}_{2}=-2 x_{1}+x_{2}-2 \operatorname{sgn}\left(2 x_{1}+x_{2}\right)
\end{aligned}
$$

Fig. 6.9 shows the initial LF used in the previous example with one valid RS corner tangency point, and again the optimal LF with the two types of valid tangency. The RAS. boundary is.

$$
\begin{gathered}
3.544 x_{1}^{2}-0.8707 x_{1} x_{2}+1.0 x_{2}^{2}+\left|2 x_{1}+x_{2}\right|=3.9941 \\
(\rho=4.17)
\end{gathered}
$$

Convergence was decraded once powell!s method hit the équal tangency surface and various initial p's were tried to get the optimun. 


\section{System 522}

A case there the optinum piecevise quadratic LF approaches a piecewise linear LF is the following

$$
\begin{aligned}
& \dot{x}_{1}=x_{2} \\
& \dot{x}_{2}=x_{1}-\operatorname{sgn}\left(x_{1}+2 x_{2}\right)
\end{aligned}
$$

Fig. 6.10 shows an initial LF $(P=I)$ compared to the optimum in which the $\dot{V}=0$ constraint forms part of the RAS boundary, $V=V_{m}$. The latter caused difficulty since the precise tangency at the optimum was not clear. (A check was made to see if $\underline{x}_{1}$ was the valid tangency where $\left.\nabla V\left(x_{1}\right)=0\right)$. Comment

Weissenberger uses a gradient method to optimize the RAS volume for 3 rd order systems. The complex method of $C_{5}$ could also be applied, with better convergence properties. The examples given here certainly show that the equal tangency property of optimal LF's is not restricted to continuous systens.

\section{The Piecewise Linear IF}

Weissenberger (66) gives an analytic expression for the optimal piecewise quadratic LF of 6.2 .9 for the system

$$
\begin{aligned}
\underline{x}=\left[\begin{array}{ll}
0 & 1 \\
p_{1} & p_{2}
\end{array}\right] \underline{x}+\left[\begin{array}{c}
0 \\
-1
\end{array}\right] \operatorname{sgn} \sigma & 6.2 .14 \\
\sigma & =d_{1} x_{1}+d_{2} x_{2}, p_{1}>0
\end{aligned}
$$

We show here that a good RAS boundary for the piecewise linear Lf of 6.2 .12 can be obtained by assuming an'equal tangency property' for the optimum LF.

Consider the LF

$$
v=\left|\underline{c}^{x} \underline{x}\right|+\left|\underline{d}^{2} \underline{x}\right|
$$


and assume sliding for 6.2 .14 is a.s., i.e.

$$
a_{1}, d_{2}>0
$$

Then without loss of generality the situation is shown in Diag. 6.1 with a contour, $\mathrm{V}=\alpha=$ const., having vertices $\mathrm{a}, \mathrm{b}$ and $\mathrm{c}$ of interest in $\sigma>0$ (by symmetry the analysis is considered for $\sigma>0$ only) and normals

$$
\begin{aligned}
& \underline{n}_{1}=\underline{d}+\underline{c} \\
& \underline{n}_{2}=\underline{d}-\underline{c}
\end{aligned}
$$

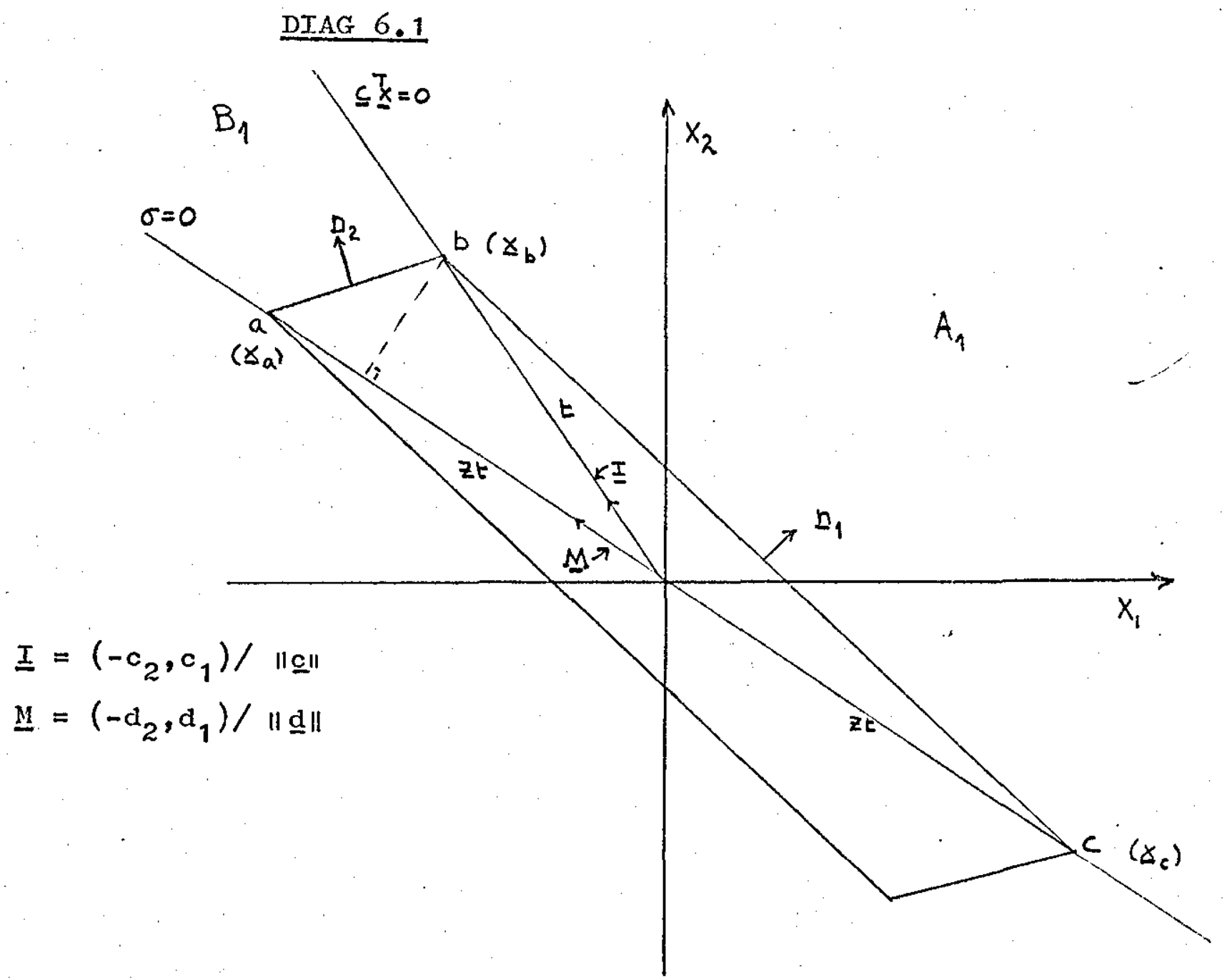

For $V=\alpha$ to be a stability boundary we require

$$
\underline{n}_{i}^{\mathbf{x}} \leqslant 0 \quad i=1,2 .
$$

at the vertices $a, b$ and $c$, or, 


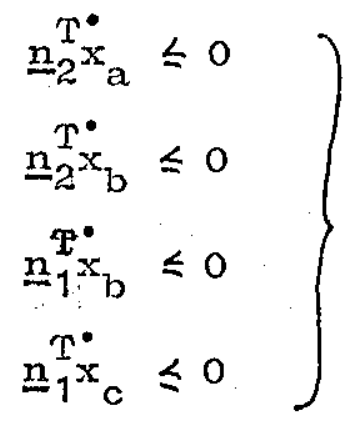

and also for sliding, which on $\sigma=0$ gives

$$
\dot{v}=\frac{n_{2}}{2^{*}}=-c^{T} x=-\frac{x_{2}}{d_{2}}\left(d_{2} c_{1}-d_{1} c_{2}\right) \text { for } x_{2}>0
$$

and $\dot{V}=\underline{n}_{1}^{T^{*}}=-n_{2^{x}}^{T^{*}}$ for $x_{2}<0$

Hence we require

$$
\mathrm{d}_{2} \mathrm{c}_{1}-\mathrm{d}_{1} \mathrm{c}_{2}>0 \quad 6.2 .17
$$

which also implies V p.d. For local stability we also require $\underline{n}_{i}^{T} \underline{b}<0$ which sives

$$
c_{2}+d_{2}>0 \text { and } d_{2}-c_{2}>0 \quad 6.2 .18
$$

Let $M$ and The unit vectors to vertices $a$ and $b$ and $z t$ and $t$ the respective distances

$$
\begin{gathered}
\left(\underline{x}_{a}=z t \underline{M}, \underline{x}_{b}=t \underline{I}, \underline{x}_{c}=-\underline{x}_{a}\right) \text { then } \\
t=\frac{\|\underline{c}\| \alpha}{\left(d_{2} c_{1}-c_{2} d_{1}\right)}
\end{gathered}
$$

and the area of $V<\alpha$ is $\rho=t \alpha /\|\underline{c}\|$ or

$$
\rho=\left(\frac{t}{\|g\|}\right)^{2}\left(d_{2} c_{1}-d_{1} c_{2}\right) \quad 6.2 .19 b
$$

The RAS is determined by the maximum $\alpha$ satisfying 6.2 .16 , or equivalently, by the minimum of the $t(>0)$ giving equality in all 4 relations of 6.2 .16 . The 4 values of $t$ are respectively 


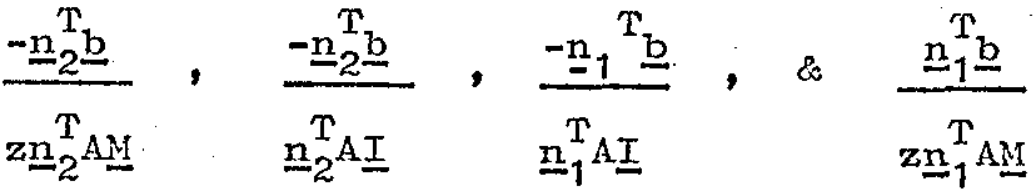

where $\mathrm{z}=\|\underline{d}\| /\|\underline{\mathrm{c}}\|$.

As the boundary $V=\alpha$ is expanded its vertices will have a first contact with the $\dot{V}=0$ constraint - in this case two RS straight lines found in regions $A_{1}$ and $B_{1}$. (Diag. 6.1). In view of the equal tangency properties which usually hold for optimum LF's we assume that the optimum boundary in this case will touch $\dot{v}=0$ at all vertices (and thus $\dot{V}=0$ on all sides of the boundary). For $\dot{\mathrm{V}}=0$ along bc we require the last two terms in 6.2.20 to be equal i.e

$$
\frac{-\underline{n}_{1}^{T} \underline{b}}{\underline{n}_{1}^{T} \underline{I}}=\frac{\underline{\underline{n}}_{1}^{T} \underline{b}}{\underline{z}_{-1}^{T} A M}
$$

which gives

$$
\left(d_{1}+c_{1}\right)^{2}-p_{1}\left(d_{2}+c_{2}\right)^{2}+p_{2}\left(d_{1}+c_{1}\right)\left(d_{2}+c_{2}\right)=0
$$

or

$$
\mathrm{u}^{2}+\mathrm{p}_{2} \mathrm{u}-\mathrm{p}_{1}=0
$$

with $u=\left(c_{1}+d_{1}\right) /\left(c_{2}+d_{2}\right)$.

Let $x=p_{2} / 2 \cdot \sqrt{p_{2}^{2} / 4+p_{1}}<0$ be the negative of onetroot of 6.2.22, then $c_{1}$ and $c_{2}$ must satisfy

$$
c_{1}+r c_{2}=-\left(r d_{2}+d_{1}\right)
$$

in order that 6.2 .21 holds (the other root gives $c_{1}, c_{2}<0$ ). The RAS boundary for such $c_{1}, c_{2}$ is determined as

$$
\mathrm{v}=\left(\frac{\mathrm{t}}{\|\underline{\mathrm{c}}\|}\right)\left(\mathrm{d}_{2} \mathrm{c}_{1}-\mathrm{c}_{2} \mathrm{~d}_{1}\right)
$$

from 6.2.19, where, from the substitution of 6.2 .23 into 6.2 .21 , we have 


$$
\frac{t}{\|\underline{c}\|}=1 /\left(p_{1} d_{2}-p_{2} d_{1}+d_{1} r\right) \quad 6.2 .24
$$

Using the fact that

$$
c_{1} d_{2}-c_{2} d_{1}=-\left(d_{2}+c_{2}\right)\left(r d_{2}+d_{1}\right)
$$

and

$$
\frac{p_{1}}{r}\left(d_{1}+r d_{2}\right)=p_{1} d_{2}-p_{2} d_{1}+d_{1} r
$$

from 6.2.23, substitution of 6.2 .24 into $6.2 .19 \mathrm{~b}$ gives the area in terms of $c_{2}$ as

$$
\rho=-\frac{x}{p_{1}}\left(c_{2}+d_{2}\right)
$$

The only restriction on $c_{2}$ is from 6.2 .18 and $\rho$ is naximized when $c_{2}=d_{2}$ Eiving the boundary

$$
\left|d_{2} x_{2}-\left(d_{1}+2 r d_{2}\right) x_{1}\right|+\left|d_{1} x_{1}+d_{2} x_{2}\right|=-\frac{2 r d_{2}}{p_{1}}=\rho
$$

(Note the first two terms in 6.2.20 are both zero, and $\dot{v}=0$ on ab) Fig. 6.10 shows this boundary for system 522 , and is the optimum (Veissenberger (66)).

\subsection{Finite Regions of Attraction For The Problem of Lure}

\section{Introduction}

We shall be concerned with the system of Lure' form

$$
\underline{x}=A \underline{x}+\underline{b} f(\sigma)
$$

$$
\sigma=\underline{c}^{2} x, \text { A stable, }
$$

where $f(\sigma)$ is a continuous non-1inear function which may leave the sector

$$
\left.\begin{array}{c}
0<f(\sigma) / \sigma<\mathrm{K}, \sigma \neq 0 \\
f(0)=0
\end{array}\right\}
$$


at some $\sigma \leqslant \sigma_{1}<0$ and/or $\sigma \geq \sigma_{2}>0$, but satisfies 6.3 .2 for $\sigma_{2} \leqslant \sigma \leqslant \sigma_{1}$. The latter restrictions often occur in practical systems where $f(\sigma)$ is unknown for Iarge $\sigma$. Even with these restrictions a region of attraction exists if the Popov condition

$$
\operatorname{Re}(1+i \omega q) G(i \omega)+\frac{1}{\mathbb{K}}>0, \quad \forall \omega \geqslant 0 \quad 6.3 \cdot 3
$$

holds for some real $q$ where

$$
G(s)=c^{T}(A-s I)^{-1} \underline{b}
$$

and $A$ is stable (Aizerman and Gantmacher ( 71$)$ ). The standard LF associated with this problem is

$$
V=x^{T} P_{x}+q \int_{0}^{\sigma} f(s) d s
$$

with $P$ p.d. If such a LF can be found which proves s.a.s. for 6.3 .2 without restriction, then with restriction, $\mathrm{v}<\mathrm{v}_{\mathrm{n}}$ will be a region of attraction if it lies in $\sigma_{2} \leqslant \sigma \leqslant \sigma_{1}$. The largest boundary $V=v_{m}$ will be tangential to $\sigma=\sigma_{1}$ or $\sigma=\sigma_{2}$ and thus

$$
\mathrm{V}=\mathrm{V}_{\mathrm{m}}=\min _{i} \mathrm{M}_{i}
$$

where

$$
M_{i}=\sigma_{i}^{2} / c^{T} p^{-1} c+q \int_{0}^{\sigma_{i}} f(s) \mathrm{ds} \quad 6.3 .7
$$

\section{An Ontimal Quadratic For the Infinite Sector}

For $\mathrm{q}=0$ and $\mathrm{K}=\infty$ a quadratic $\mathrm{V}=\underline{x}^{\mathrm{T}} \mathrm{P} \underline{\mathrm{x}}$ (if it exists) giving $\dot{V} \leq 0$ without restriction on $f$ in 6.3 .2 is given by

$$
\underline{P} \underline{b}=-\underline{c} / 2
$$

and

$$
A^{T} P+P A=-Q \leqslant 0
$$


(Then $\dot{V}=-\underline{x}^{T} \underline{x}-\sigma f(\sigma) \leq 0$ ) Weissenberger (72) has proposed the optimization of the volume of region 6.3 .6 ,

$$
\rho=\max _{p \in \bar{P}} \pi\left[\frac{v_{m}^{n}}{d(p)}\right]^{\frac{1}{2}}
$$

with $\bar{P}:(P / 6.3 .8$ and 6.3 .9 satisfied), to obtain the optimal quadratic when the popov condition holds, ensuring $\overline{\mathrm{P}}$ is non-empty. Walker and McClamroch (73), hovever, suggest a single LF obtained via the Kalman construction procedure (67). For the general case $(q \neq 0)$ the assumptions made are (1) the pairs $(\Lambda, \underline{D})$ and $\left(A^{T}, \underline{c}\right)$ are completely controllabie (see (43)) and (2) the Popov condition holds for some real $q$ with $a(q \Lambda+I) \not 0$. Then a LF of form 6.3 .5 exists where $P$ solves

$$
A^{T} P+P A=-\underline{u} \underline{u}^{T}
$$

and $\underline{u}$ is a real vector determined by writing

$$
\operatorname{Re}(1+i \omega q) G(i \omega)+\frac{1}{K}=\left|\frac{\theta(i \omega)}{d(i \omega I-A)}\right|^{2} \quad 6.3 \cdot 12
$$

and setting

$$
\underline{u}^{T}(s I-A)^{-1} \underline{b}=\sqrt{z}-\theta(s) / d(s I-A) \quad 6.3 .13
$$

( $\theta$ is a real polynomial of degree $n$ and $z=1 / K-q c^{T} \underline{b}$. Kalman chooses roots of $\theta(\omega)$ to have neg. real parts). The following relation now holds between the two approaches above:

\section{Theorem 6.1}

For $n=2$ the maximizing $P$ in 6.3 .10 is determined via the Kalman construction procedure i.e. 6.3 .11 
With a genexal $\Lambda, \underline{b}$ and $\subseteq$ the proof is long and tedious. However, since it is assuned the pair (A, b) is completely controllable a transformation exists (see Lefshetz (43)) transforming 6.3 .1 to cononical form without changing the problem. Hence without loss of generality ve assume the system

$$
\underline{\dot{x}}=\left[\begin{array}{cc}
0 & 1 \\
-b & -a
\end{array}\right] \underline{x}+\left[\begin{array}{c}
0 \\
-1
\end{array}\right] f(\sigma) \quad 6.3 .14
$$

Here

$$
G(s)=\left(c_{2} s+c_{1}\right) /\left(s^{2}+a s+b\right)
$$

and the relation for $\theta$ in 6.3 .12 reduces to

$$
x+\omega^{2} t=\theta(i \omega) \theta(-i \omega)
$$

with $r=c_{1} b>0$ and $t=a c_{2}-c_{1}>0$. The two choices of $\theta$ are $\theta(i \omega)=\sqrt{r}+i \omega \sqrt{t}$ and $\theta(j \omega)=\sqrt{r}-i \omega f$. With the former, $\underline{u}=-(\sqrt{r}, \sqrt{t})$ giving

$$
\underline{u u}^{x}=\left[\begin{array}{cc}
x & \sqrt{x t} \\
. & t
\end{array}\right]
$$

It is easy to show that the $Q$ corresponding to the optimal $\mathrm{P}$ in 6.3 .10 is just this. For 6.3 .8 implies $P$ of the form

$$
p=\left[\begin{array}{ll}
\alpha & c_{1} / 2 \\
& c_{2} / 2
\end{array}\right] \quad \text { with } a(p)=\left(2 \alpha c_{2}-c_{1}^{2}\right) / 4
$$

and

$$
Q=\left[\begin{array}{cc}
r & -\left(\alpha-\frac{1}{2}\left(a c_{1}+b c_{2}\right)\right) \\
t & t
\end{array}\right]
$$

Hence we require to minimize a subject to $Q$ p.d. or

$$
-\sqrt{r t}<\alpha-\frac{1}{2}\left(a c_{1}+b c_{2}\right)<\sqrt{r t}
$$

Hence the optimal $Q$ is that in 6.3 .15 . 
Interestingly, the $P$ minimizing the area in 6.3 .10 corresponds to the other choice of $\theta$.

The result was also shown valid for specific 3 rd order examples and is conjectured to hold for general n.

Variation of $p$ with $q(K=\infty)$

The system 6.3 .1 will be globally asymptotically stable if the modified frequency response (X vs. Y) with

$$
G(i \omega)=X(\omega)+i Y(\omega) / \omega
$$

lies to the right of the Popov line

$$
X-q Y+\frac{1}{F}=0
$$

for some $q$ in the $X-Y$ plane. A range of such $q, q_{2}<q<q_{1}$ will usually exist and the question arises of whether a search for the $q$ giving the best stability region in 6.3 .6 is profitable, with $\mathrm{V}$ obtained via the Kalman construction procedure. We consider 4 second order examples for the infinite sector which give different types of frequency response and range of $q$.

For system 6.3 .14 and LF 6.3 .5 the modified frequency response is a convex curve and the Popov lines tangential to it give the range of $q$. Let $\alpha=a c_{2}-c_{1}$ and $\beta=c_{2} b-c_{1} a$ then the tangency values give

$$
q_{1}=\frac{1}{\beta^{2}}\left(\alpha \beta+2 b c_{1} c_{2}\right)+\sqrt{4 c_{1} c_{2} b+\left(\alpha \beta+c_{1} c_{2} b\right)}
$$

and

$$
q_{2}=
$$

$$
\text { n }
$$

The following ranges then hold

Case 1: $\alpha \leq 0, \beta>0 ; q_{2}<q<q_{1}$

Case 2: $\alpha \geqslant 0, \beta>0 ; 0 \leqslant q<q_{1}$

Case 3: $a<0, \beta<0 ; q_{2}<q<\infty$

Case 4: $\alpha>0, \beta<0 ; 0 \leqslant q<\infty$ 
Fig. 6.11 to 6.14 show examples of the 4 cases for $f=\sigma-\sigma^{3} \quad(-1<\sigma<1)$. Evidently, marked increases in the size of stability regions are found for some $q$ and in only one case (Case 3 ) is the optimum q near one of the extreme points $q=q_{1}$ or $q=q_{2}$ (i.e $q=10^{4}$ in Fig. 6.13). 


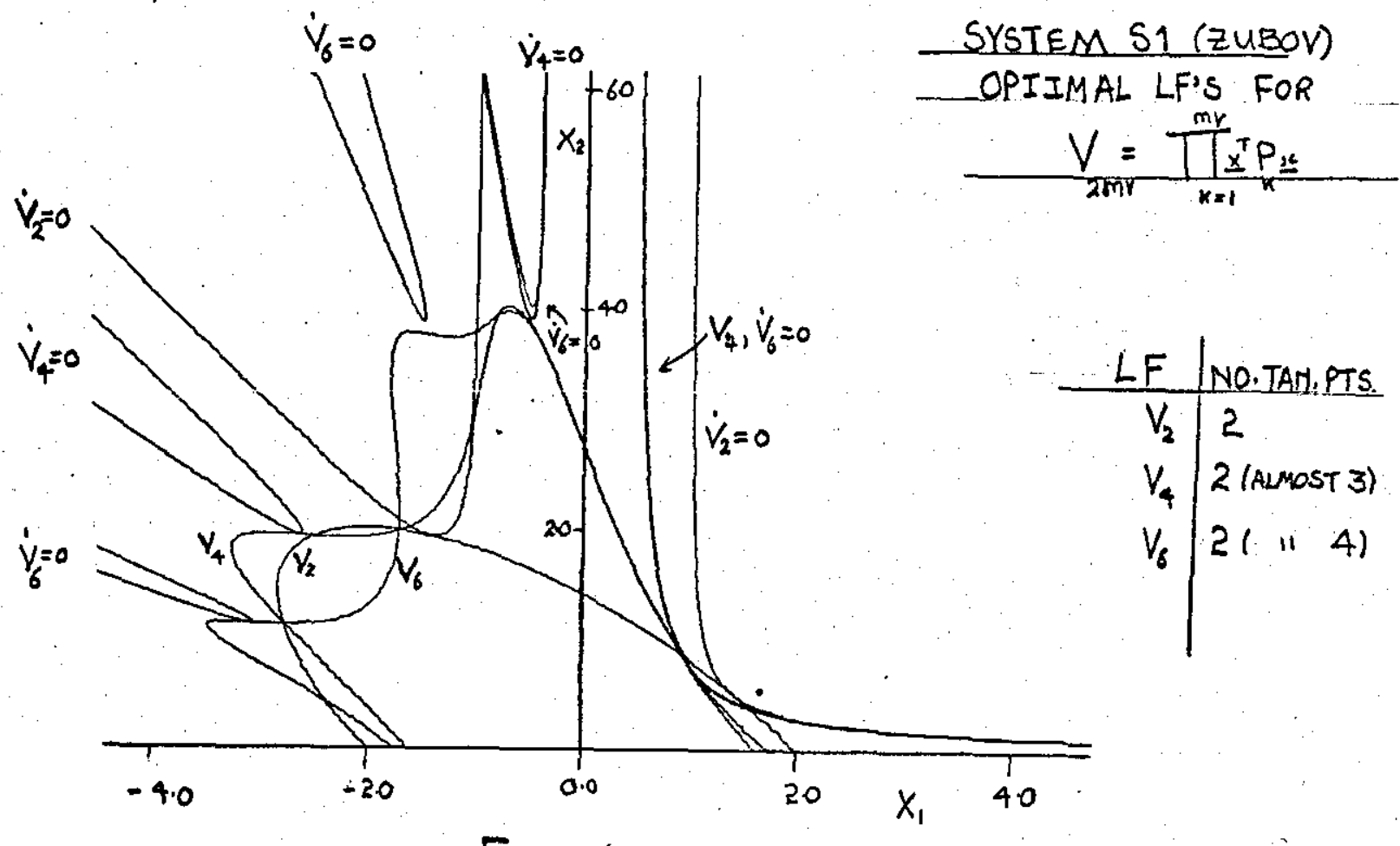

FIG.6.1.

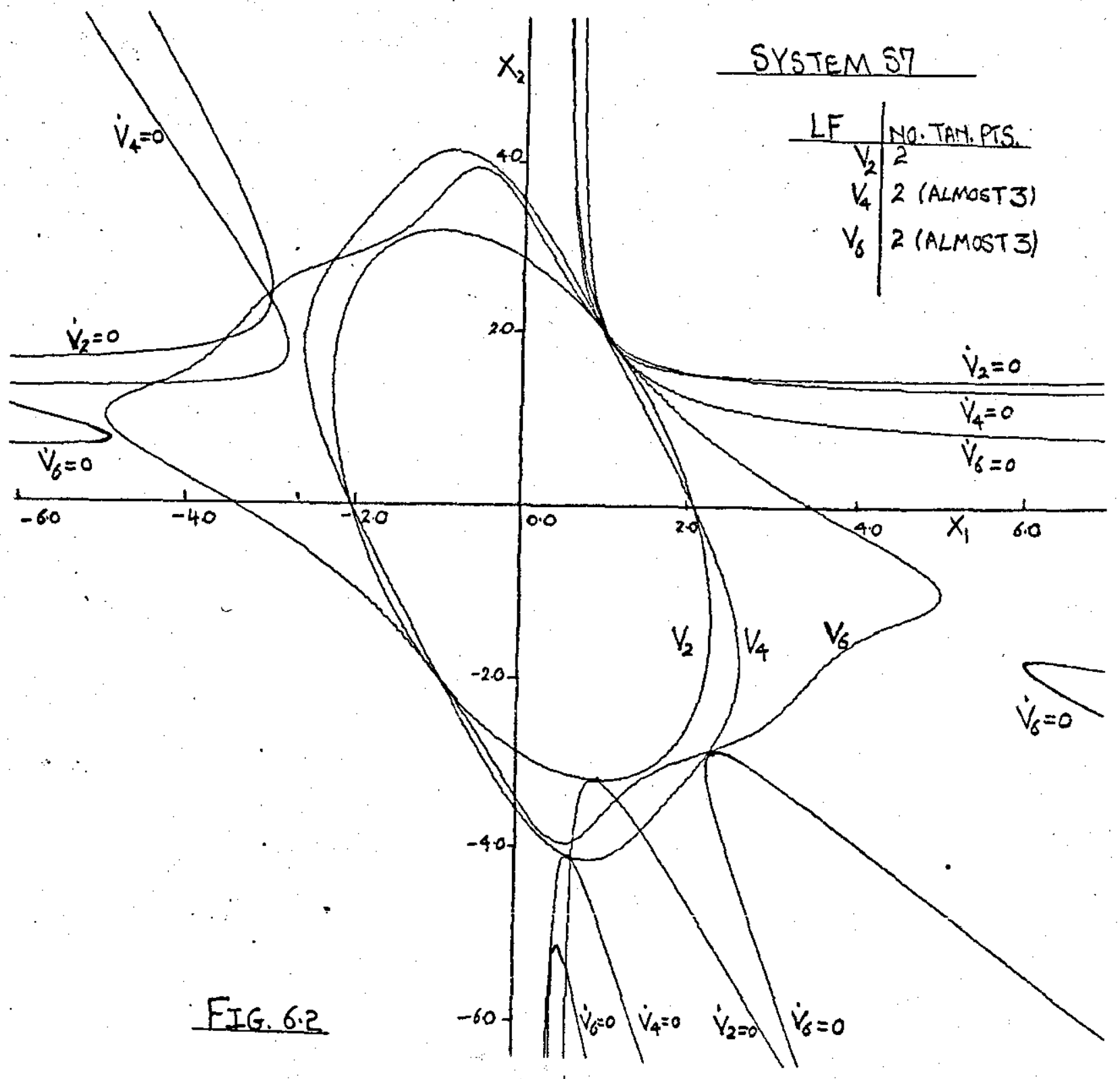




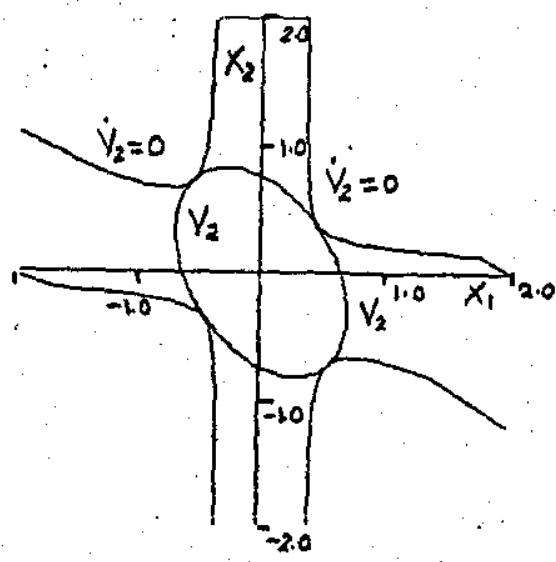

$\frac{V=V_{2}}{2 \text { VALID RS TAM. BS. }}$

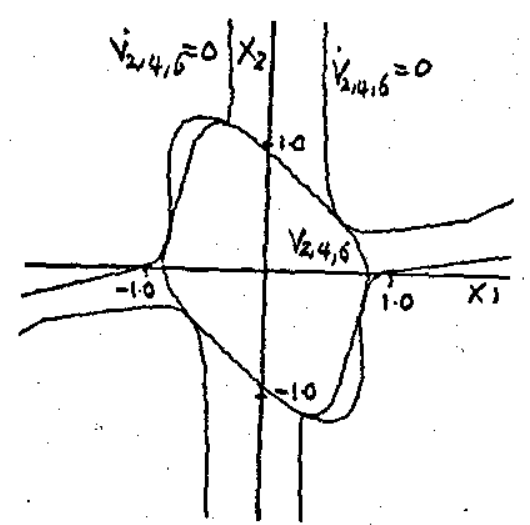

$\frac{V=V_{2}+V_{4}+V_{6}}{3 \text { VALID RS TAN.PTS. }}$

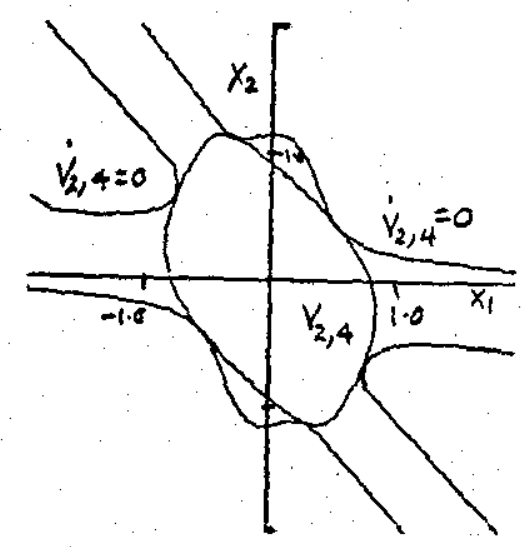

$\frac{V=V_{2}+V_{4}}{3 \text { VALID RS TAN.PTS. }}$ OPTIMAL CONTOURS FOR SERIES LE $(6.1 .3)$

- SYSTEM S2 (VAM DER POL, E=10)

\begin{tabular}{|c|c|c|c|}
\hline$V$ & $P$ & NO OF & TIME \\
\hline$V_{2}$ & 1.44 & 70 & 760 \\
\hline$V_{2,4}$ & 1.798 & 184 & 883 \\
\hline$V_{2,416}$ & 1.805 & 290 & 3050 \\
\hline * RESTARTED
\end{tabular}

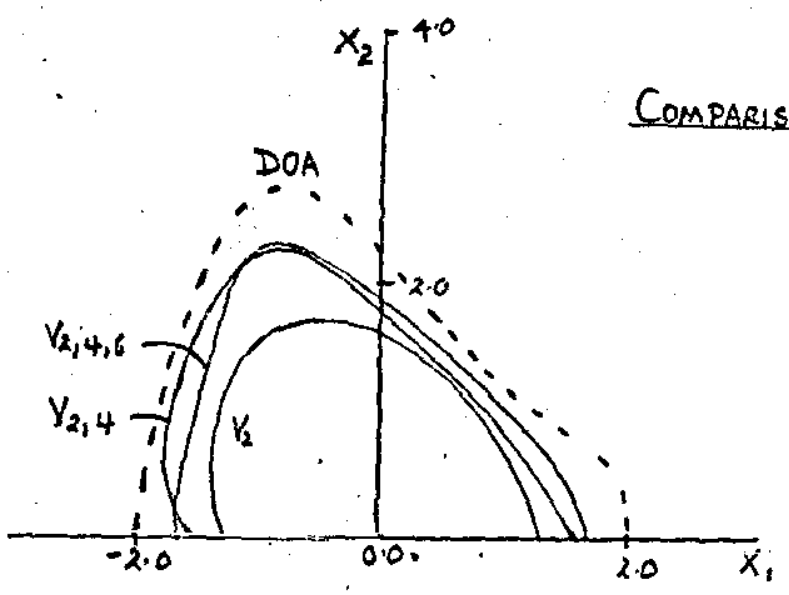


156

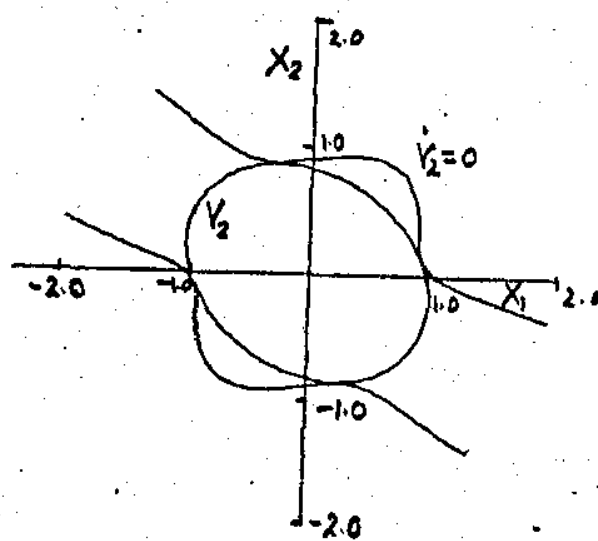

$$
\frac{V=V_{2}}{2 V A L I D \text { TAM. PIS }}
$$

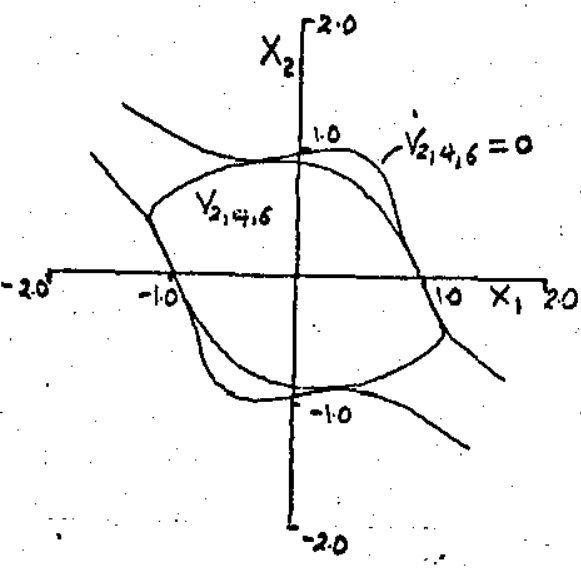

$$
\frac{V=V_{2}+V_{4}+V_{0}}{2 \text { VALID TAN PTS. }}
$$

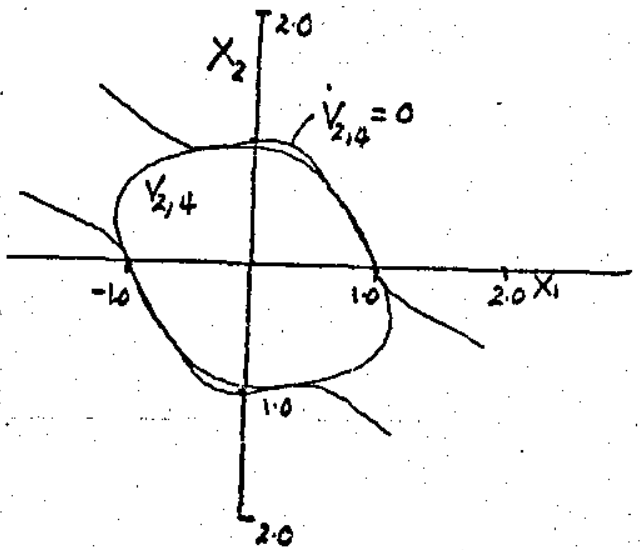

$$
\frac{V=V_{2}+V_{4}}{2 \text { VALID TAM PTS }}
$$

- OPTIMAL CONTOURS FOR SERIES LE (6.13) SYSTEM SIP

\begin{tabular}{|c|c|c|c|}
\hline$V$ & $\begin{array}{c}\text { P } \\
\text { (AV RAD })\end{array}$ & $\begin{array}{c}\text { NO OF } \\
\text { FESS }\end{array}$ & $\begin{array}{c}\text { TIME } \\
\text { (MILL/SEO }\end{array}$ \\
\hline$V_{2}$ & 0.903 & 70 & 630 \\
\hline$V_{2,4}$ & 0.997 & 136 & 1024 \\
\hline$V_{2,4,6}$ & 1.00 & 288 & 2592 \\
\hline
\end{tabular}

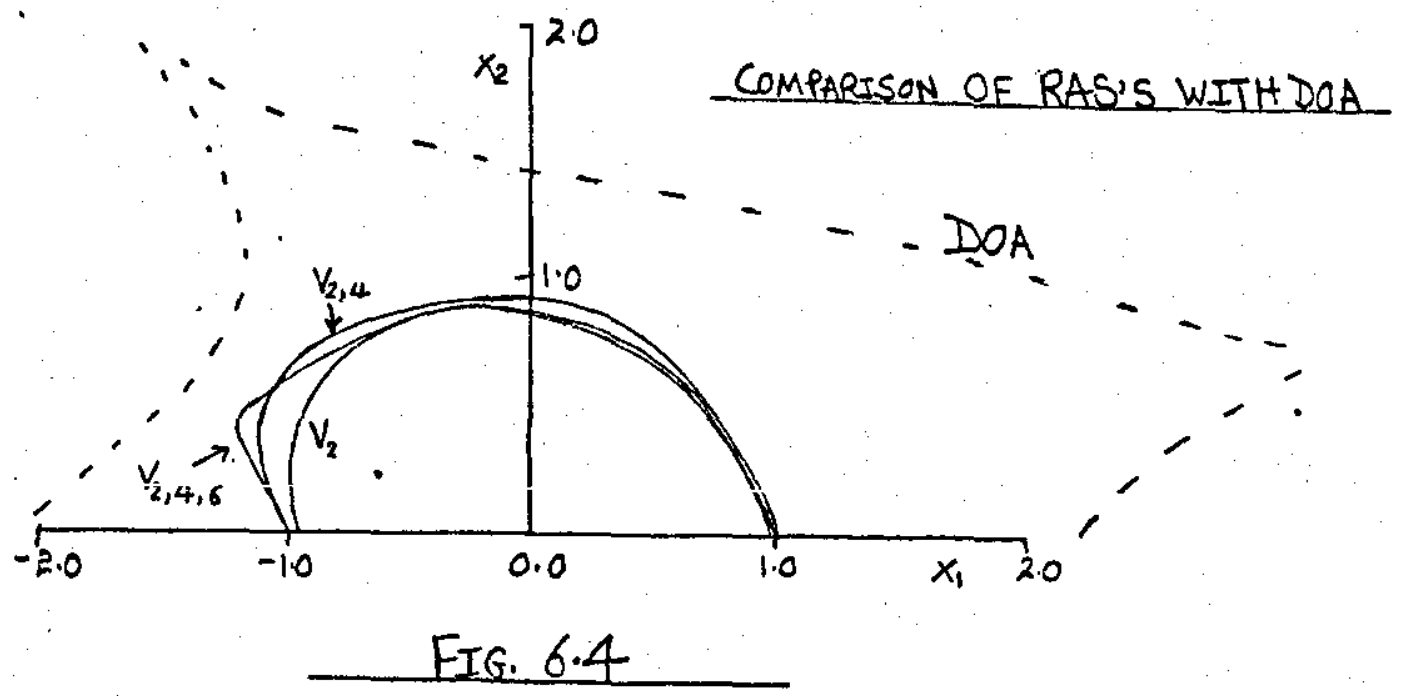


157

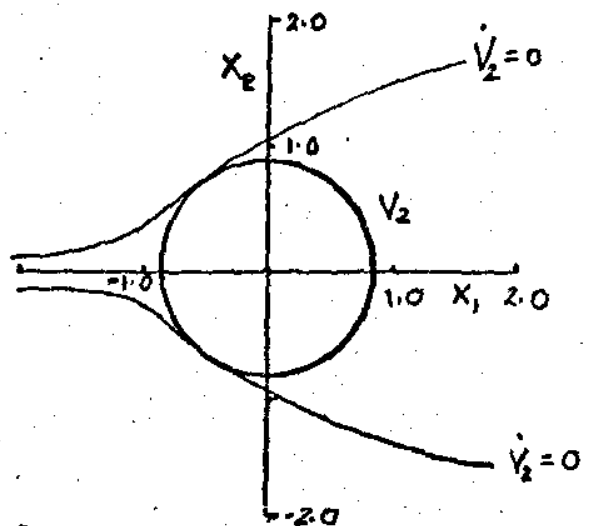

$V=V_{2}$

2 VALID TAR PTS.

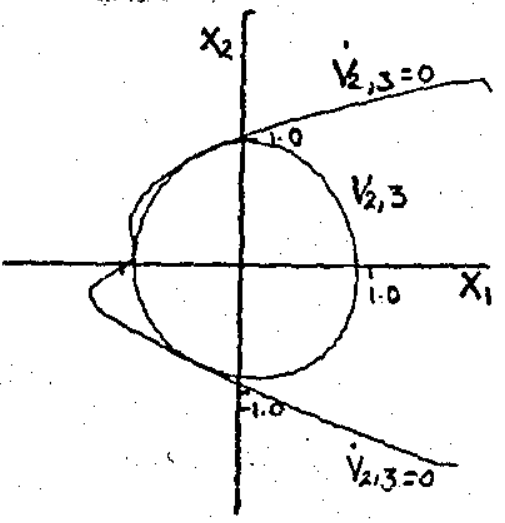

$\frac{V=V_{2}+V_{3}}{3 \text { WALD TAN PTS. }}$

- OPTIMAL CONTOURS for SERIES LE $(6.1 .3)$ SYSTEM S19

\begin{tabular}{|c|c|c|c|}
\hline$V$ & AX PARD & NO I OF & TIME \\
\hline$V_{2}$ & 0.86 & 40 & 174 \\
\hline$V_{2, x}$ & 0.903 & 101 & 590 \\
\hline$V_{2,3,4}$ & 0.951 & 318 & 1900 \\
\hline
\end{tabular}

3 VALID TAN. PTS.

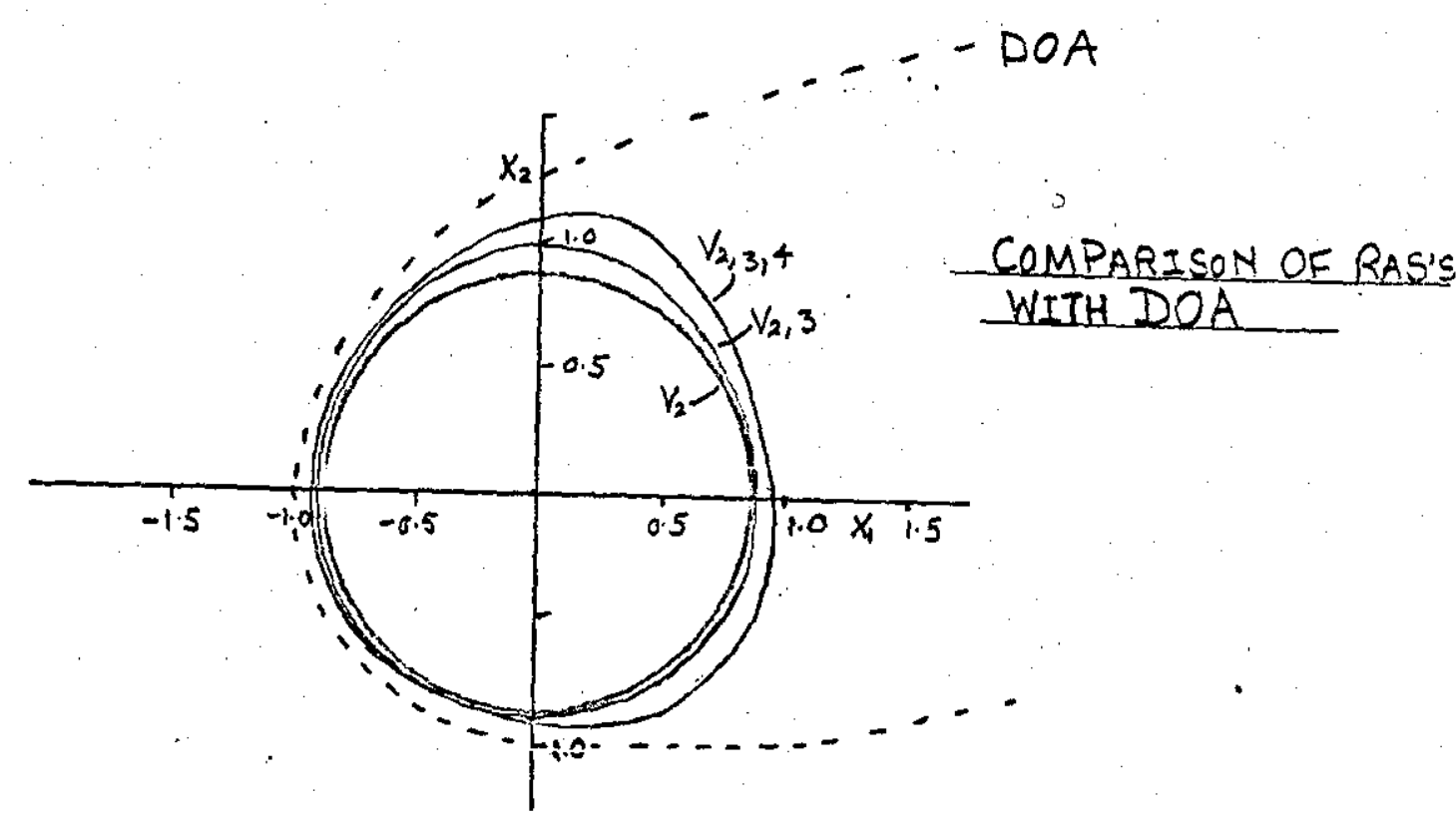

FIG .6.5 


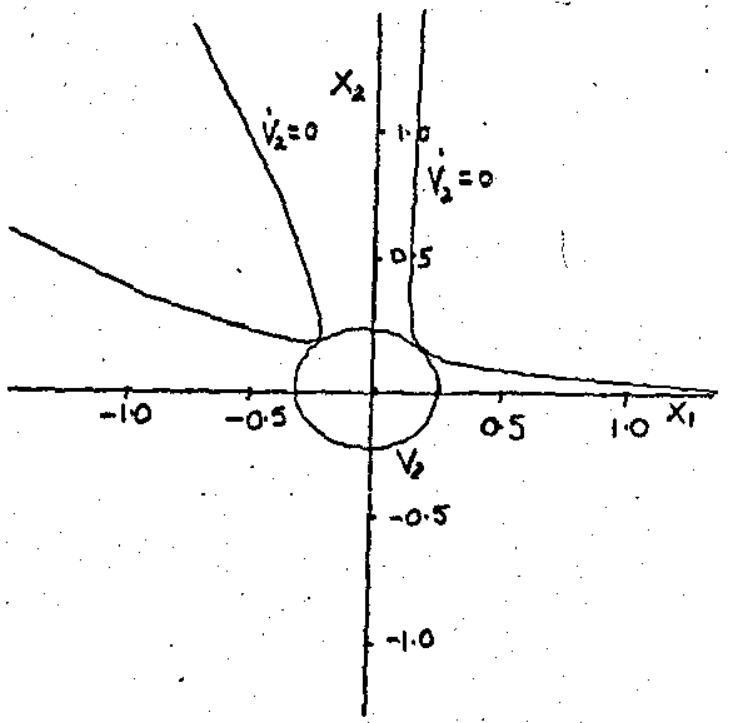

$V=V_{2}$

2 VALID TAN.PTS.

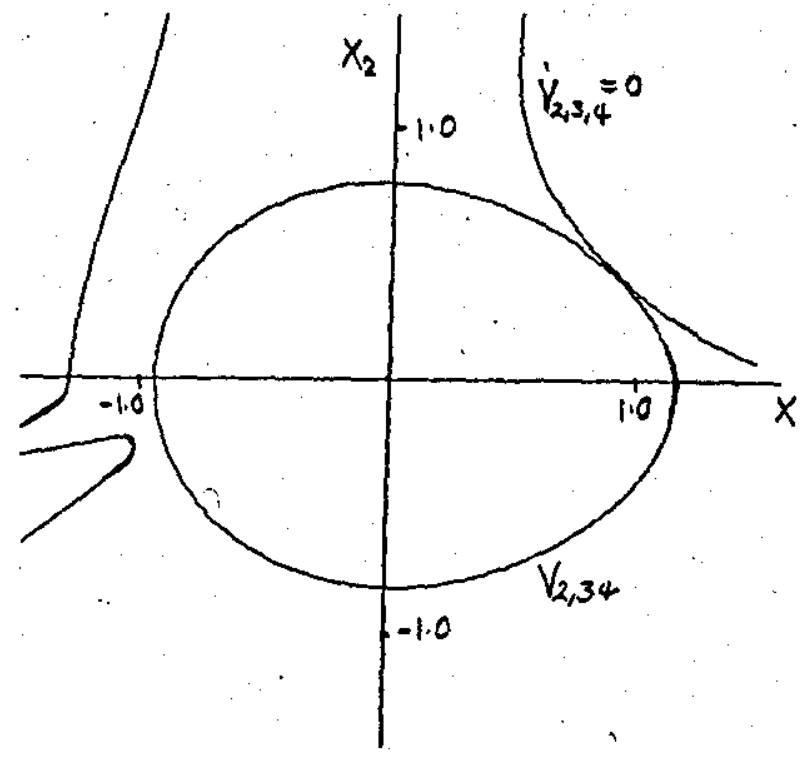

$V=V_{2}+V_{3}+V_{4}$

1 VALD TAW.PT.

(NOT CONVERGED) $x_{2}$

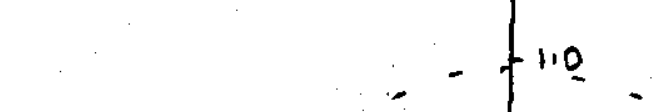

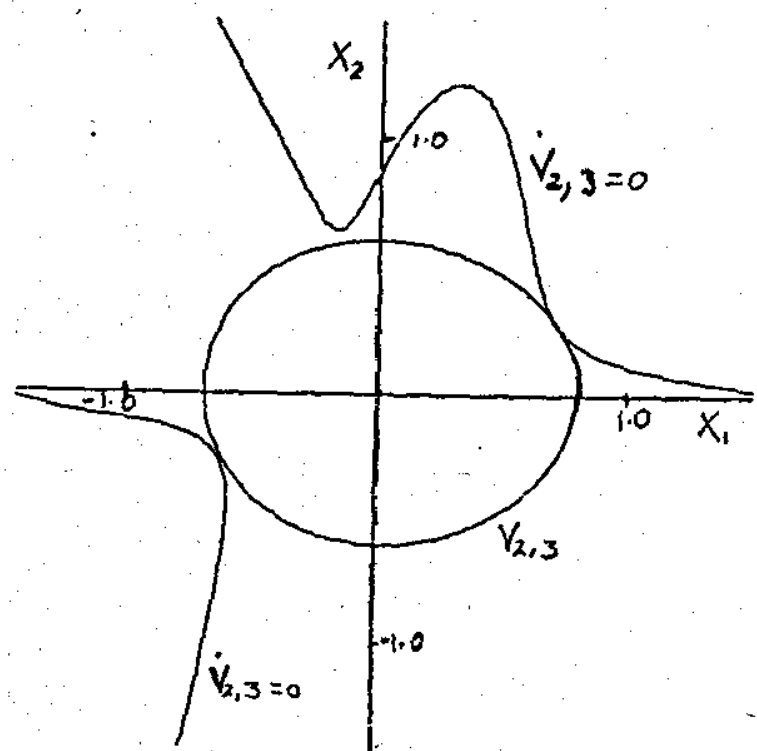

$\frac{V=V_{2}+V_{3}}{\frac{2 V A L T D \text { TAN, PTS. }}{\text { (NOT CONYERGED) }}}$

OPIIMAL CONTOURS OF SERIESLE $(6.13)$

SYSTEM (Yu) 513

\begin{tabular}{|c|c|c|c|}
\hline$V$ & $\begin{array}{c}P \\
\text { (AV.RAD) }\end{array}$ & $\begin{array}{l}\text { NO. OF } \\
\text { FE'S }\end{array}$ & $\begin{array}{c}\text { TIMEE } \\
\text { (MILLISEC) }\end{array}$ \\
\hline$V_{2}$ & 0.265 & 70 & 600 \\
\hline$V_{2,3}$ & 0.865 & 200 & 2400 \\
\hline \multirow{2}{*}{$V_{2,3,4}$} & 0.795 & 226 & 2700 \\
\cline { 2 - 5 } & 0.906 & 59 & $5000^{*}$ \\
\hline
\end{tabular}

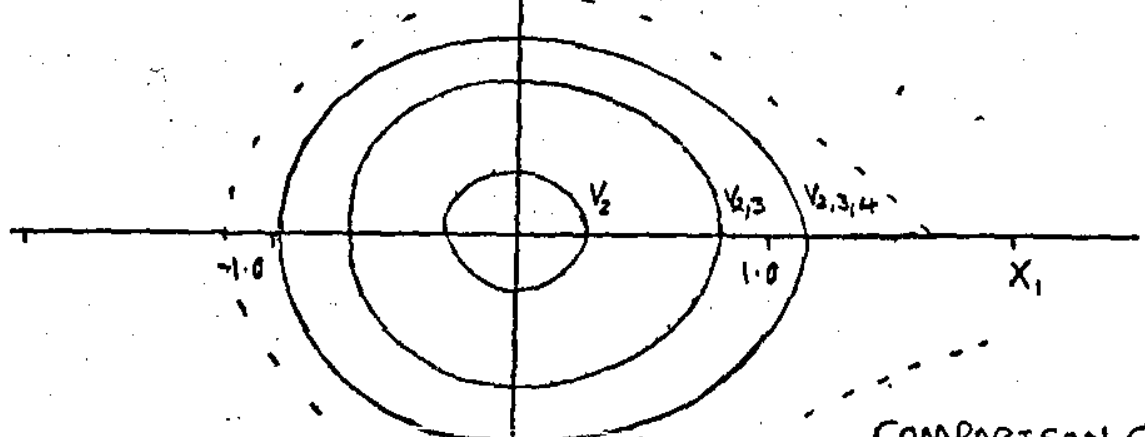

COMPARISON dF RAS'S WITH DOA 
159

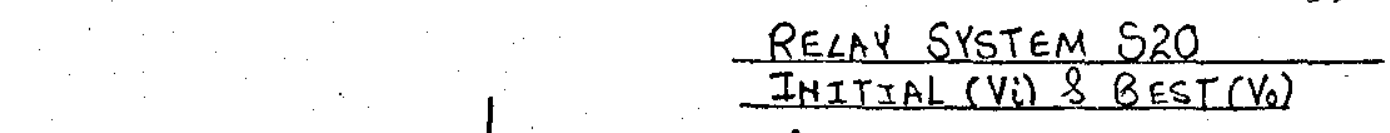

RELEWISE QUADRATIC
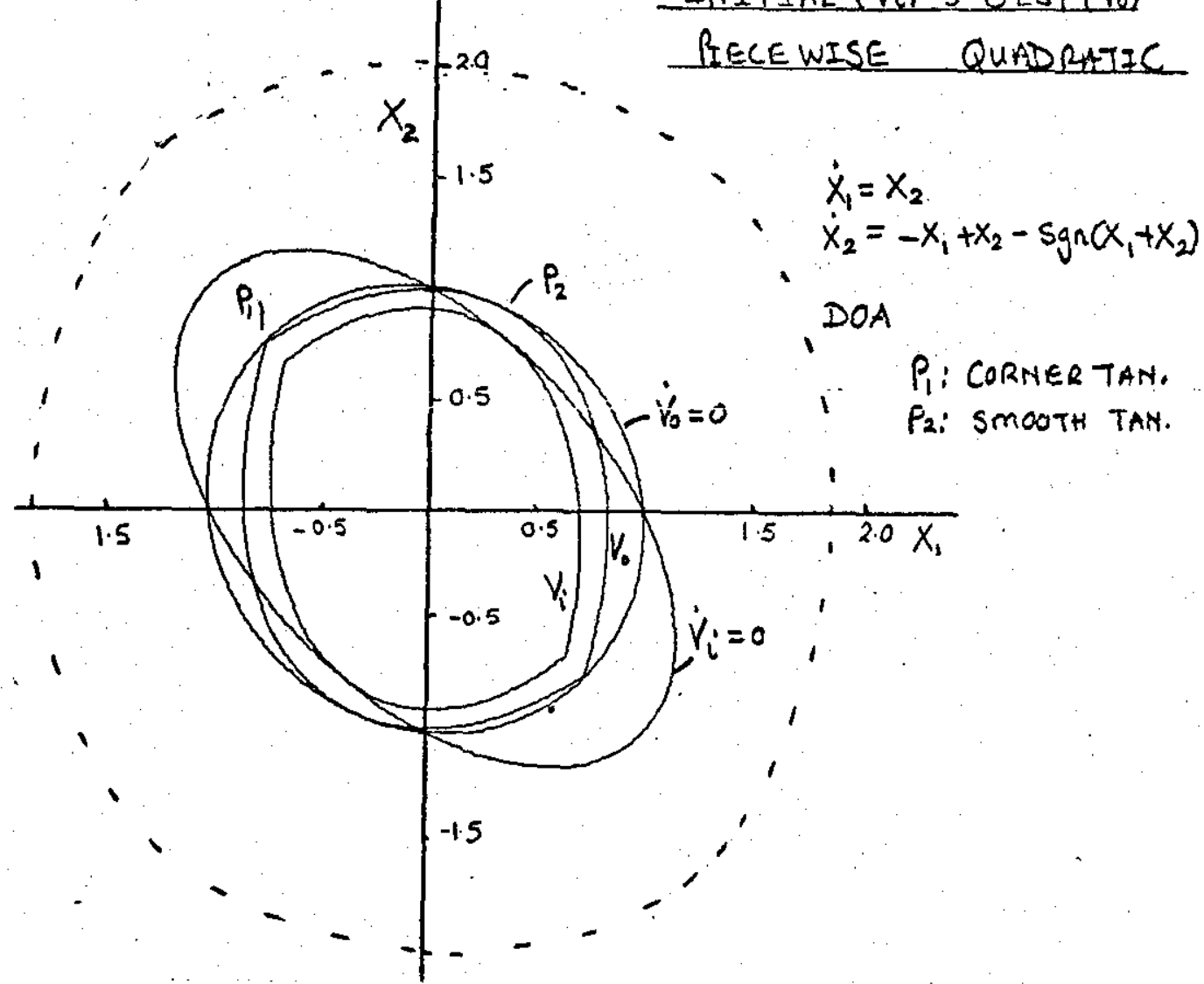

$$
, D O A
$$

$P_{1}$ : CORNER TAN. $P_{2}$ : SMOOTH TAN.

FIG .6.7

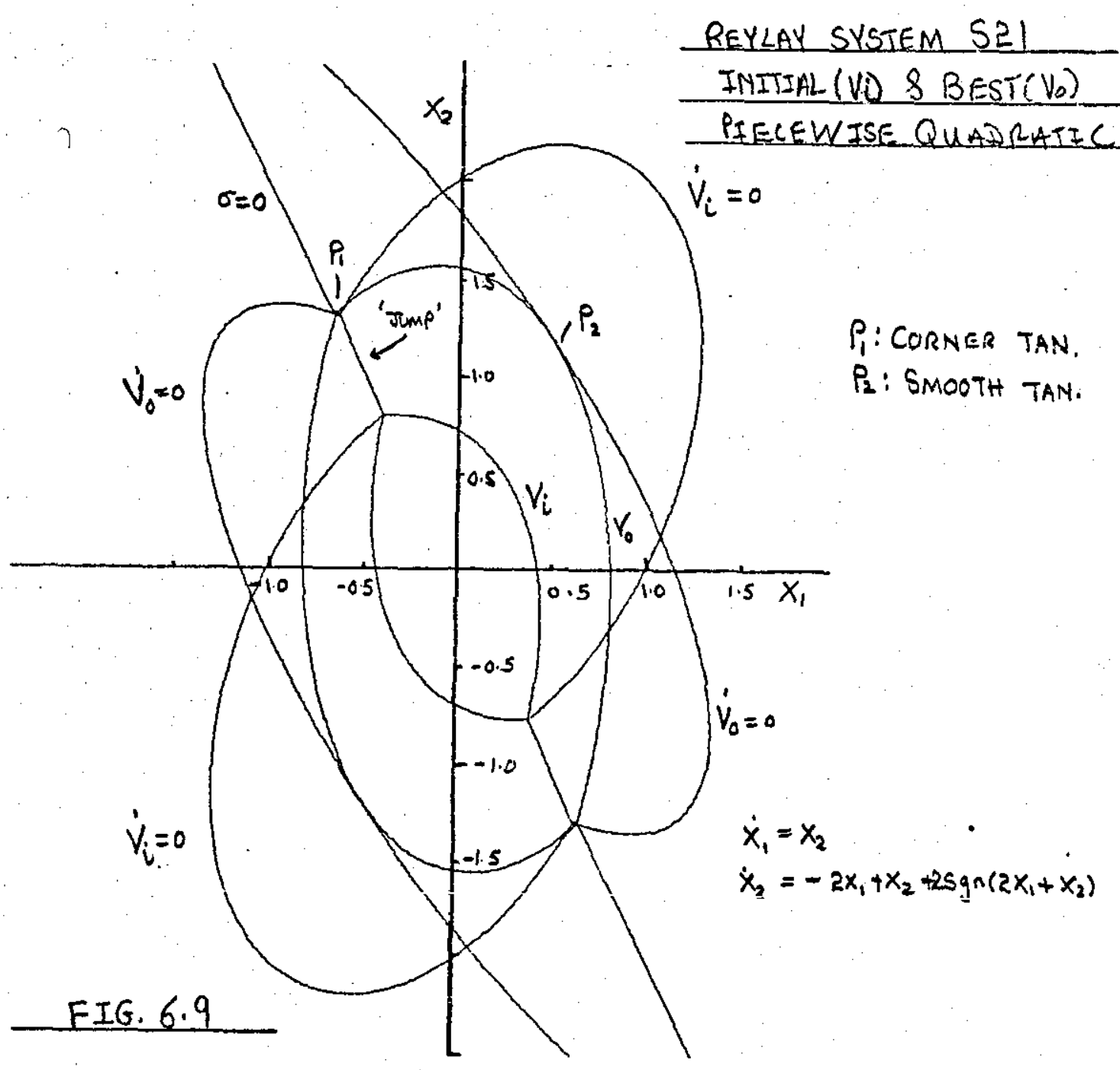




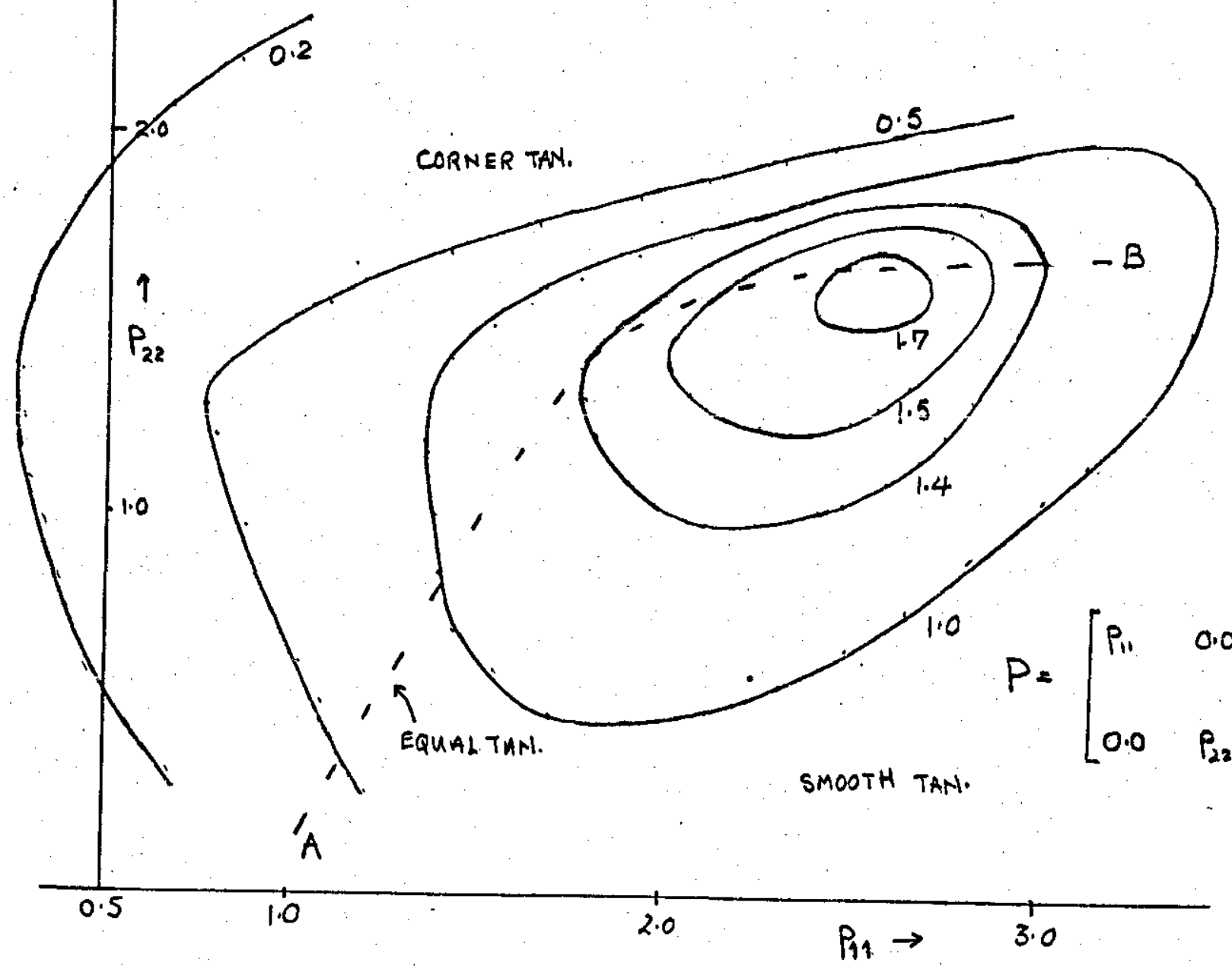

AREA CONTOURS FOR PFECEWISE QUNDRHTIC, SYSTEM SZO (REYLAY)

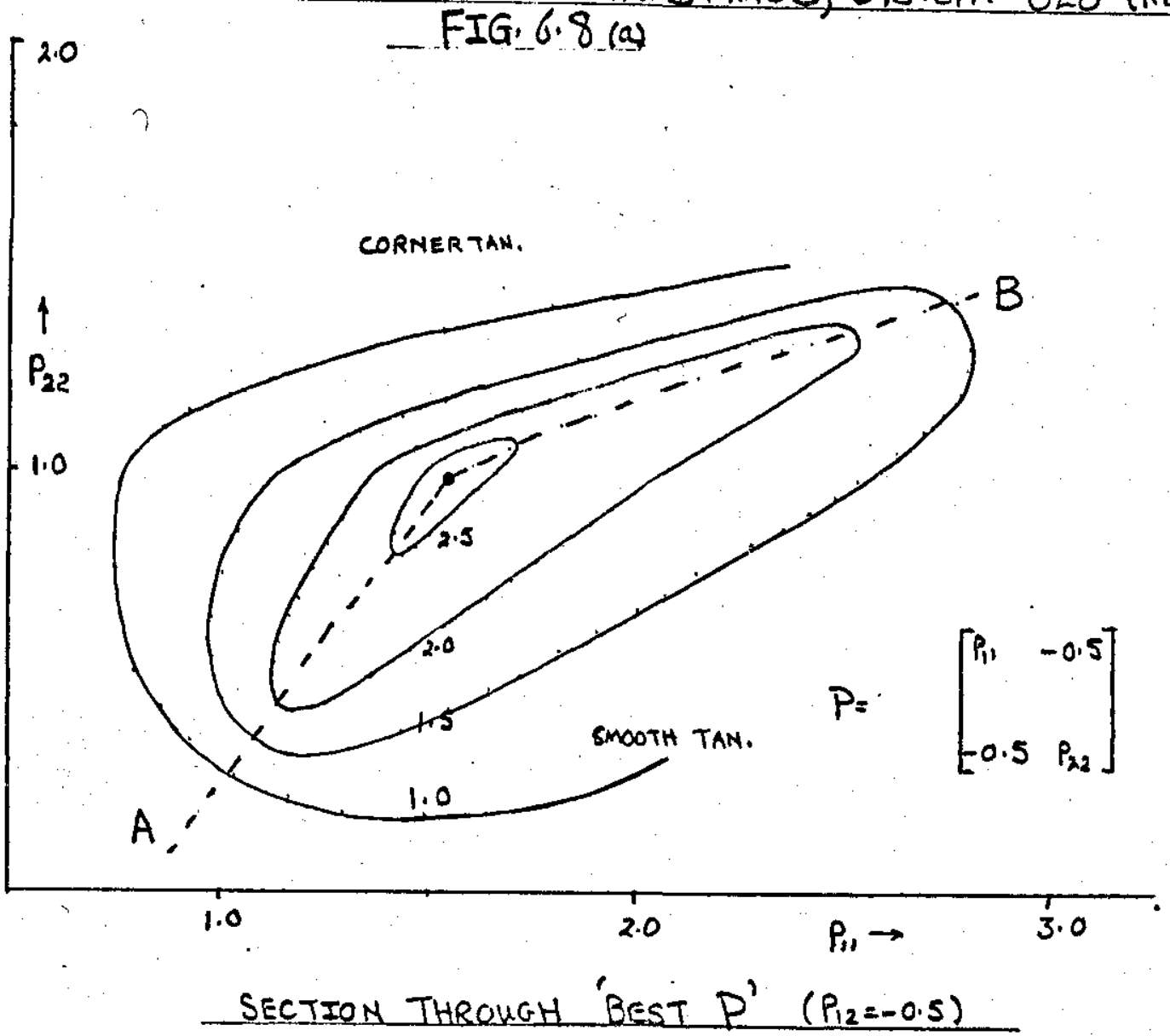




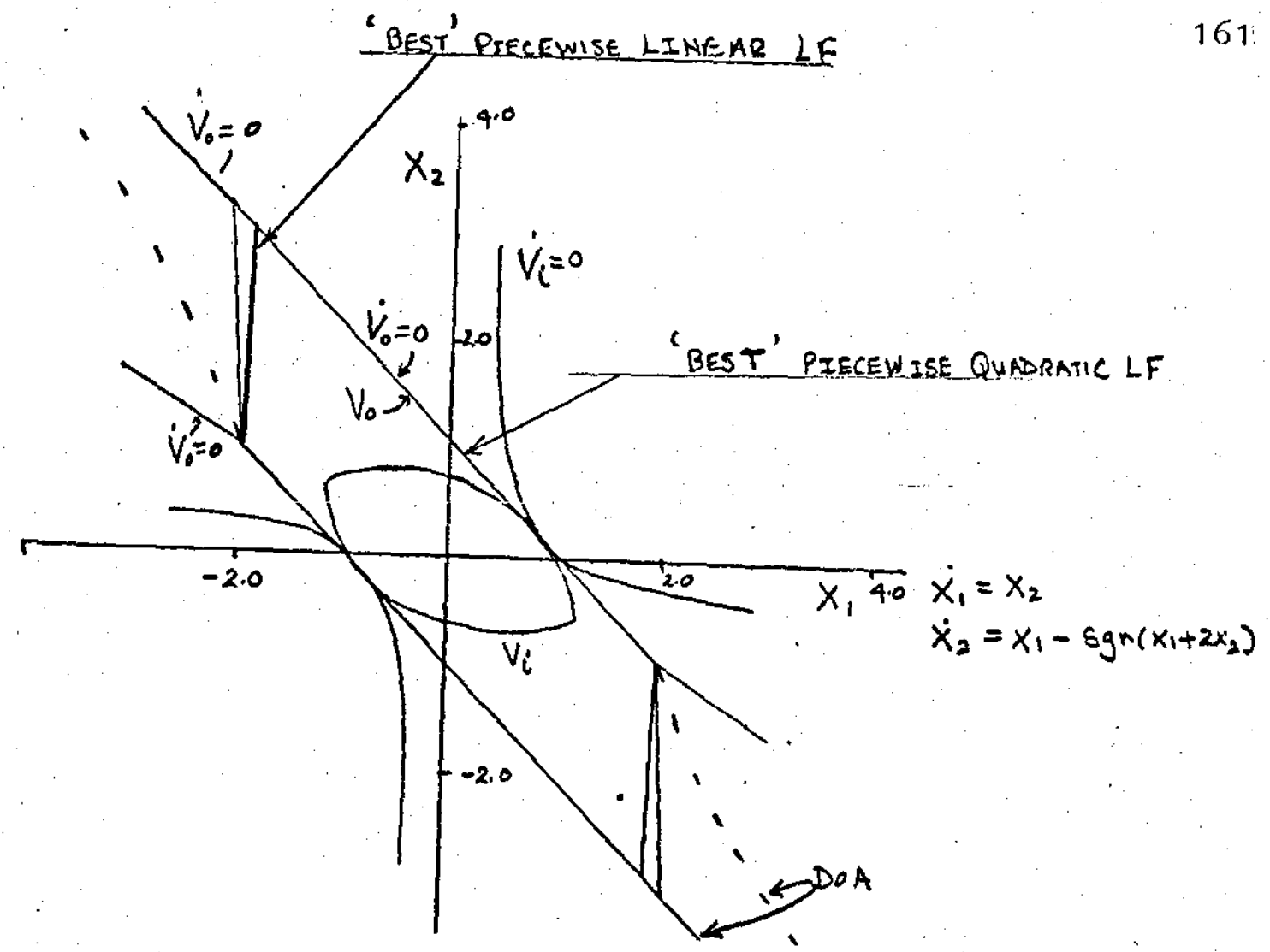

INIIIAL (Vi) \& BEST (Vo) PIECEWISE QUADRATIC SYSTEM S??

$F I G .6 .10$

$$
V=\underline{x}^{\top} P \underline{x}+\int_{0}^{\sigma} f(s) d s=V_{m}
$$

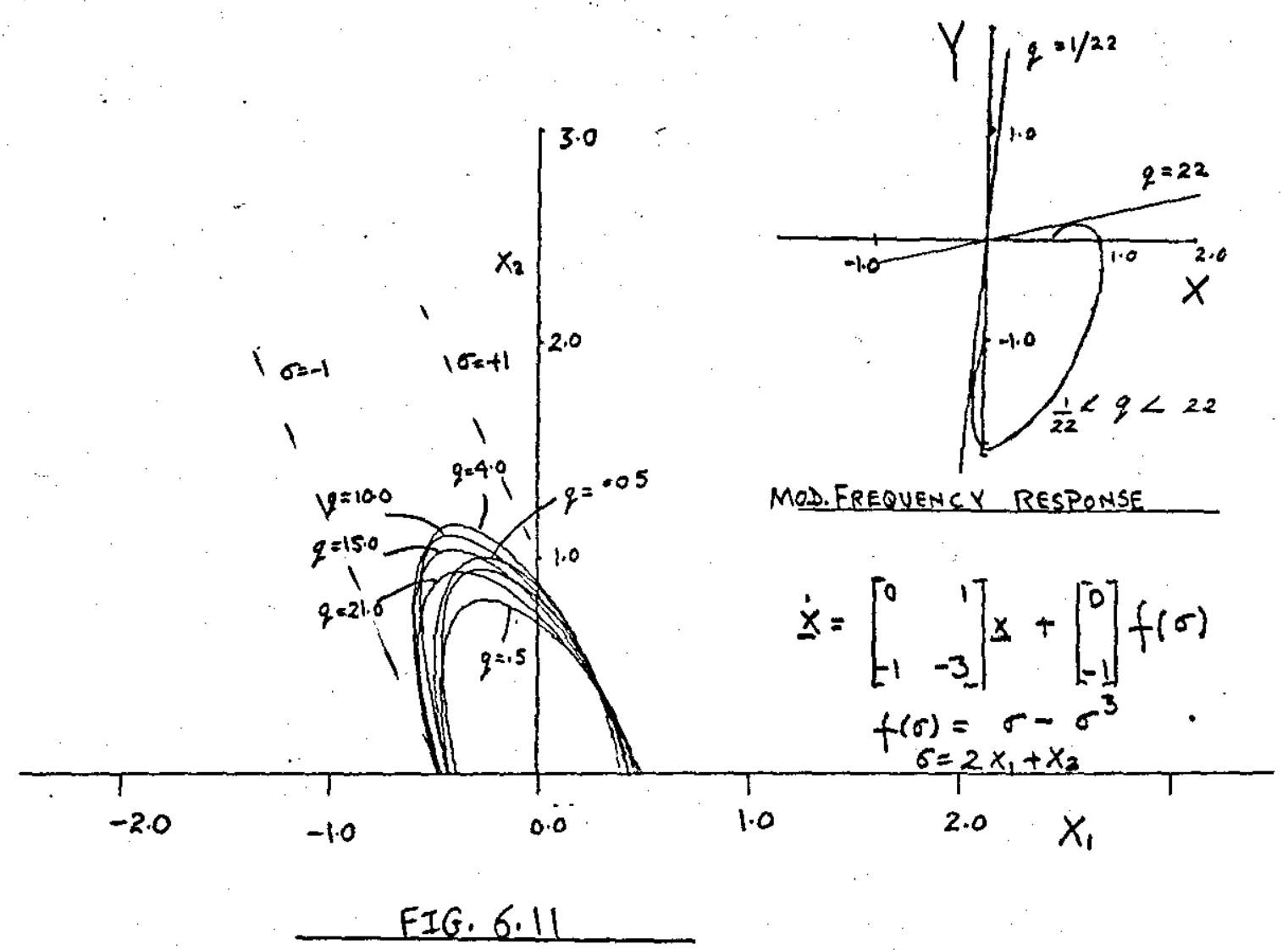


162
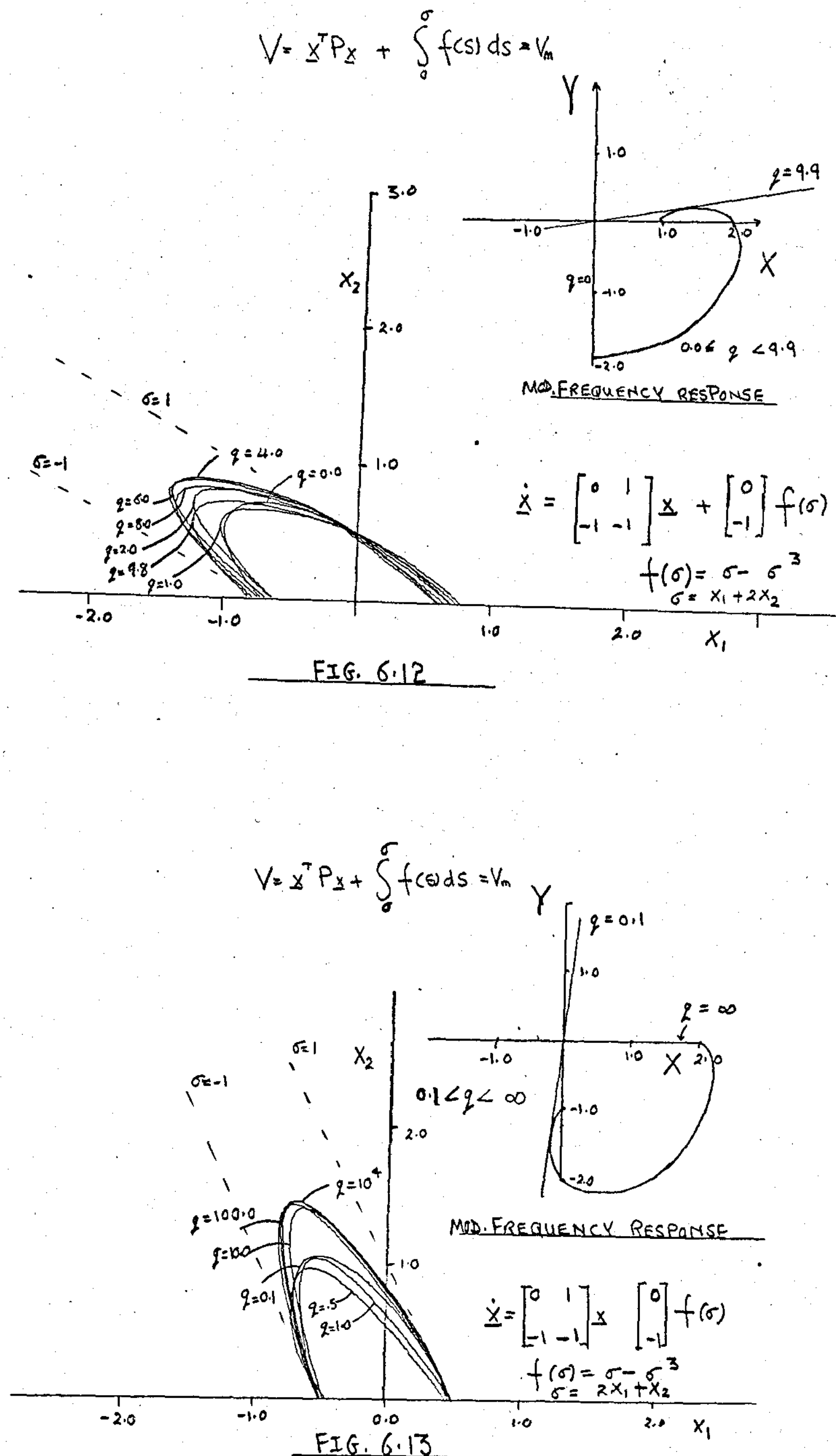
$x=x^{\top} P_{x}+\int_{0}^{\sigma} f(s) d s$

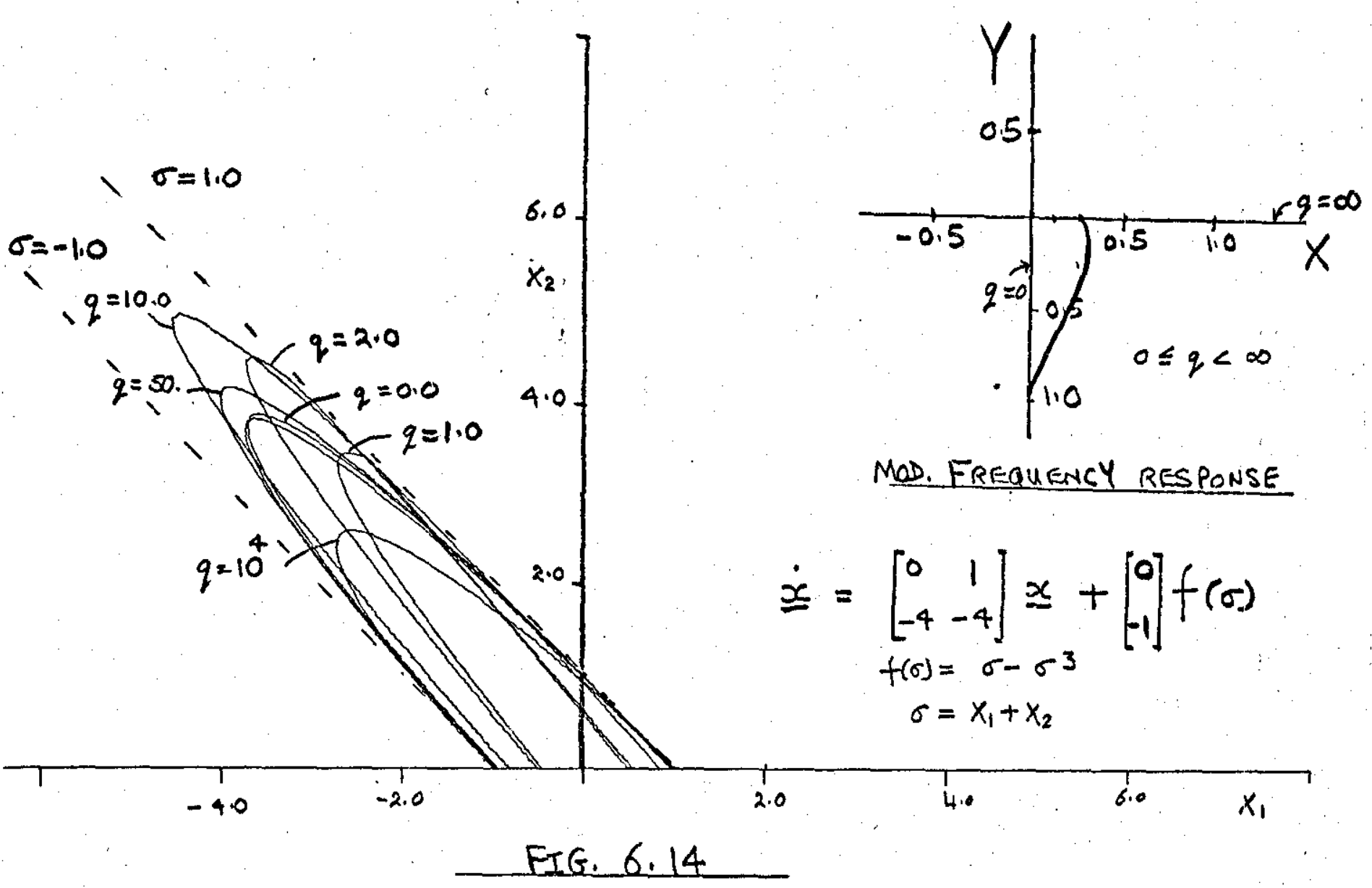


CONCLUSIONS 


\section{Conclusions}

The continuous theme throughout this thesis has been the search for improved results when applying Lyapunov functions to autonomous non-linear systems. We intend here to bring together the main points arising from this search and add some suggestions for future work.

The problem of improving a crude RAS or an estimate of the transient response of a non-linear system was considered in Chapter 2. For the two forms of system considered the rate at which the trajectories approach the origin can be estimated through quantities $\alpha$ or $\beta$ where $\alpha=\eta / 2-\sqrt{\mu} c_{0}$ and $\beta=\eta / 2-\mu_{c_{2}}$; where $\alpha^{-1}$ and $\beta^{-1}$ behave as 'time-constants' of the systems. The sub-optirrum problem of maximizing $\eta\left(\mathrm{m}\left(\mathrm{P}^{-1} \mathrm{Q}\right)\right)$ and then minimizing $\mu(P)(M(P) / m(P))$ over the space of p.d.s.matrices $\bar{Q}$ which results, was solved for a special case where A was in companion form with reai eigenvalues. A conjecture and a bound of Wiberg were also proved invalid.

Although the theory of condition numbers is well established there are a surprisingly small number of useful bounds that are obtainable for $\mu(P)$. The main drawback of the ones proposed, namely, $F(P), F\left(P^{-1}\right)$ and that of 2.6 .14 , is the calculation of $d(s)$, although simplification arises with $A$ in companion form.

The numerical work shows that the procedure of minimizing $\mu(P)\left(P^{-1}=(S D)(S D)^{*}\right)$ over the extended space of hermitian matrices Eives no improvenent over that of the real matrices. The results in Table 2.1 show the extra effort in computing the estimates $\left\||S|\left|S^{-1}\right|\right\|$ and $\mu\left(S S^{*}\right)\left(\left\|s_{i}\right\|=1\right)$ may be worthwhile. The evidence in Table 2.2 causes some doubt on the sub-optimum problem as simple choices of $P$ or $Q$ often give larger values of $\alpha \operatorname{or} \beta$. 
Although the choice of a quadratic Lyapunov function gives rise to many simplifications in obtaining bounds for the transient response, the optimizations of quantities such as $\alpha^{-1}$ or $\beta^{-1}$ are essentially independent of the non-linear parts of the system thus giving crude bounds.Unless one considers a very special type of system,LF's incorporating the non-1inearity are hard to find.

An analytic solution of the zubov eqn. in Chapter 3 would give the optimum result in that the exact DOA would be prescribed, $\mathrm{V}<1$ (regular equation). The impossibility of analytic solutions in general has led to the Zubov construction procedure with its non-uniform convergence of the RAS to the DOA. The analytic example given has thrown some light on the reason for this. However, the problem of determining whether the convergence of. the RAS to the DOA is always non-uniform when the region of convergence of the pover sexies LF is a subset of the DOA, would appear intractable analytically, and may be so numerically in view of the difficulty in finding the region of convergence. The breakdown of the difference method near the DOA boundary when solving Zubov's PDE, is disappointing. It is due. to the possibility of the analytic solution for $V$ not being defined outside the DOA (Example 3.6 .1 shows that with $\phi=r^{2}$, $V$ is defined everywhere, whereas for $\phi=10 x^{2}+y^{2}$, only in the DOA). The validity of the difference approach is also questionable and breakdowns occur. A more critical comparison of the methods of Troch, Burnand \& Sarlos and Davidson and Cowan may prove interestinß̊. 
In the author's view the work in Chapters 4,526 contain the essential result of this thesis. It has been shorn, both from an analytical and numerical standpoint, that given, a class of Lyapunov functions the optimal LF, $V$ say, can have more thax one valid tangency point with its constraint contour, $\dot{v}=0$. Although the number of such points depends upon the system considered, for a quadratic LF, the property can be suitably defined as 'property A'. Analytic evidence has shown the intractability of proving that the property A always holds for autonomous systems not asymtotically stable in the whole. However, the numerical evidence gives some overwhelming evidence for such a property. A contrary result has not yet been encountered Defining a similar property for higher degree LI's is difficult due to the number of special cases - for example, a system giving only one tangency point for any LF - but the view that multiple valid tangency is a general phenomenon encountered with any class of suitable LF's is certainly supported by the evidence in this thesis.

In the following paragraphs we give the main points of each chapter.

The problem of RLS determination and its associated search for the global minima of a constrained function is a big obstacle in using Lyapunov's direct method. With this in mind Chapter 4 investigates the simplest class of Lyapunov functions, 
the quadratics. Even with very simple non-linear second order systems RAS determination is impossible. The systems studied show this in detail but in two cases optimal quadratics are obtained, one of which gives a lead to the equal tangency property, property A.

It is hoped that the second and higher order optimal quadratic algorithms will be of use to future researchers. They have certainly shown the existance of property A for a number of second and third order examples where accuracy was needed. Future work would be to compare some of the best optimization methods (e.g Fletcher and Lill, Nelder and Mead, Powell and DCS) on a varied selection of optimal quadratic problems. The degrading of the search directions near an equal. tangency curve as for Powell's method, should affect these other methods to varying degrees. Future optinization al gorithms should be chosen or developed on the fact that, although a good direction of search is along the equal tangency curve or surface, the RAS determination for a quadratic corresponding to a point on this curve is also degraded because of the extra effort in verifying the valid tangency point.

An extension of Chapter 4 is the problem of optimizing over the class of quadratic LF's in order to obtain a large region of the origin in which trajectories have a certain exponential stability. For example we maximize the volume inside a boundary $\mathrm{V}=\mathrm{v}_{\mathrm{m}}$ in which also $\dot{\mathrm{v}}+2 \lambda \mathrm{V}<0\left(2 \lambda<\mathrm{m}\left(\mathrm{p}^{-1} \mathrm{Q}\right)\right)$. The problem is similar to the one of the optimal quadratic and it is easily shown that for system S1 equal tangency exists. In Chapter 5 the superiority of the 'complex' optimal quadratic algorithm over that of Davidson and Kurak was mainly due to some unsatisfactory steps in the latter algorithm, namely, solving the constrained problem by Rosenbrock's hill-climber and 
minimizing the eccentricity of the initial stability boundary. The main reason for the latter step was to avoid local minima of the objective function $\rho$ (volume). In our experience local minima were rarely encountered. The main point was that of a bad initial quadratic which in the space of elements of $\mathrm{L}$ $\left(P=L^{T} L\right)$ meant that the search for the optjmm engaged an equal tangency surface someway from the optimum with little hope of reaching it.

It would be advantageous to find a large class of systems which for a quadratic LF have only one RS tangency point where the above maladies do not occur, A member is the system 54 .

The penalty function approach to the quadratic RAS probiem in Chapter 5 has given good results. Further experience with the penalty function method of rletcher and Lill (63) for high order systems is required in view of the claims of the method by the authors.

The extensions made in Chapter 6 of obtaining quite general optimal LF's for non-linear systems, including relay systems, give conclusive numerical evidence for the equal. tangency phenomenon being a general one, and not solely attributed to quadratics.

The problem of determining optimal LF's in the series form $(6.1 .3)$ has numerous pitfalls. Besides those of the quadratic, the rreater number of valid tangency points for $\mathrm{mv}$ increasing, the possible open nature of the $\mathrm{V}_{\mathrm{mv}}=$ constant boundaries and the vanishing of $\nabla_{\mathrm{mv}}$ at tangency, all cause serious problems in RAS determination for mv $>2$. From a purely mathematical standpoint the question of why multiple valid tangencies occur is striking. As the degree of the Li increases so do the degrees of freedom of its contours, as shown in Figures 6.1 to 6.6 , but the direct relationship between these 
contours and those of $\dot{\mathrm{V}}_{\mathrm{mv}}=0$ at tansency, is obscured by the 1. RAS determination. Finding the number of valid tangency points a' priori for a given LF of derree mv and a given system is an unsolved problem. It is hoped that the numerical evidence presented in this thesis has made some contribution to its solution.

By assuning an equal tangency property a useful suboptimun piecewise linear LF has been obtained in Chapter 6 for a second order relay system. Perhaps this idea could be extended to other systems. The proof for general $n$ that the Kalman construction procedure gives the optimal quadratic.would be worthwhile in view of the ease in obtaining the region of attraction.

The numerous practical systems have shown that the optimal RAS of a quadratic often givesa good estimate of the DOA when the latter is radially symmetric, but that in general it is poor, especially for an open DOA (Figures 4.4, 5.3, 5.4, $5.5,6.4$ and 6.5 show this) Hewit (2), after comparing the best RAS's for a number of constructed Lyapunov functions (those of Krasovski, Ingwerson, Zubov and Szegoi) concluded that Lyapunov's direct method was unsatisfactory when applying numerical construction procedures to it. The presence of the equal tangency phenomenon for second and high order systems and its associated convergence problems, gives even more evidence against Lyapunov's direct method as a tool for estinating the DOA.

We can conclude from the work in this thesis that the search for optimal results has been profitable and that applications range from simple autonomous systems to systems of discontinuous right-hand sides. It is hoped that the optimal phenomena shown have contributed to a better understanding of 
Lyapunov's direct method. 


\section{REFIRENCISS}

1. Zubov V.I, Methods of A.M.Iyapunov and Their Applications, Noordhoff Ltd, Gronigen, Netherlands, 1964.

2. Hewit J.R., A Comparison of Numerical Methods in Non-Linear Stability Analysis, Ph.D Thesis, 1968.

3. Rodden J.J, Numerical Applications of Lyapunov Stability Theory, J.A.C.C, Session IX, Paper 2, Stanford University, California, June 1964.

4. Margolis S.G., Vogt V.G., Control Engineering Applications of V.I.Zubov's Construction Procedures for Lyapunov Functions, I.E.E.E, AC-3, 1963, pp104-113.

5. De Sarker A.K, RAO N.D; Zubov's Method and Transient-stability Problems of Power Systems, I.E.E. Proc. Vo1 118, pp1035-1040, Aug. 1971.

6. Yu Y.N and Vongsuriya, Non-Linear Pover System Stability Study by Lyapunov Function and Zubov's Method, I.E.E.E, Trans. Power App. Syst., Vol PA.S86, pp1480-1485, Dec. 1967.

7. Ferguson D.R, Generation of Zubov's Construction Procedure for Lyapunov Functions, Elec. Letters, Vol. 6, no.3, Feb. 1970.

8. Froberg C.E., Introduction to Numerical Analysis, AddisonWesley, 1965.

9. Richtmyex R.D. and Morton K.W., Difference liethods for Initial-Value Problems, Interscience Pub., 2nd Ed. 1969.

10. Burnand G., Sarlos G., Determination of the Domain of Stability, J.Math. Anal. Appl., Vo1 23, pp714-722, 1968.

11. Davidson E.J, Cowan K.C., A Computational Method for Determining the Stability Region of a second-order NonIinear Autonomous System, Int. J. Control, 1969, Vo1.9, No.3, pp349-357. 
12. Trocil I, The Evaluation of the Domain of Attraction of Non-linear Control Systems with Hybrid Computing Systéms, Procd. $5^{\text {th }}$ IFAC Cong., Part 4 No.32.1.

13. Kormanik J, Li C.C, Decision Surface Estimate of Non-1inear System Stability Domain by Lie Series Method, TEEE, AC-17, oct. 1972, pp666-669.

14. La Salle J.P. \& Lefshetz S., Stability by Lyapunov's Direct Method with Applications, Acad. Press New York (1961).

15. Hahn W., Theory and Applications of Lyapunov's Direct Method, Prentice-Ha11, 1963.

16. Kalman $\mathrm{R} . \mathrm{H}$ \& Bertrain J.E, Control Synthesis and Design via the Second Method of Lyapunov, Part 1, A.S.M.E, D82, p371, 1960.

17. Lehnigk S.Hn, Stability Theorems for Linear Niotions, PrenticeHall 1966.

18. Barbashin E.A, Introduction to the Theory of Stability, Wolten-Noordhof̂, 1970.

19. Vogt W.G., Transient Response from the Lyapunov Stability Eqn: Joint Automatic Control Conference, 1965.

20. Barnett S., Solution, of some Algebraic Probleins Arising in the Theory of Stability and Sensitivity of Systems, with Paxticular Reference to the Lyapunov Matrix Equation, Ph.d Thesis (1967) loughborough University.

21. Barnett S. \& Storey C., Matrix Methods in Stability Theory, Nelson, 1970 .

22. Bauer F.I, Optimally Scaled Natrices, Numerische Mathenatik S.73-87, 1963.

23. Wiberg D.M, A Simple Bound for Stable Non-linear Systems with Input, Int. J. Control, Vol.6, No.4, 343-350, 1967. 
24. Lewis D.C., Taussky a, On the Eigenvalues of The Lyapunov Matrix Operator, J. Math. Phs., 1, 234, 1960.

25. Bass R.W, Control Synthesis \& Optimization, NASA Langley Research Centre, Aug. 1961.

26. Bellman R.E., Stability Theory of Diff. Eqns., New York : McCraw-Hil1, 1953, p35.

27. Marcustlo Haynsworth E, Bound for the P Condition No. of Matrices with Positive Roots, Nat. B. Stds., 65B, No 1, $1961,13-14$.

28. Gautshi W., On Inverses of Vandermonde 2 Confluent Vandermonde Matrices, Num. Math. 4, 113-123, 1962.

29. Reiss G., Inversion of the Vandermonde Matrix, I.E. B.E, AC-12, p793, 1967 .

30. Marcus M., Minc H., A Survey of Matrix Theory and Natrix Inequalities, A11yn and Bacon, Boston, 1964.

31. I.S. Pace \& S. Barnett, Comparison of Numerical Methods for Solving Lyapunov Matrix Eqns., I.J.C, Vo1 15, pp907-915, 1972.

32. Rosenbrock H.H., An Automatic Method For Finding The Greatest or Least Value of a Function, Comp. Jnl., 3, pp175-84, 1960 .

33. Powell M.J.D., An Efficient kethod of Finding the Minimum of a Function of Several Variables Vithout Calculating Derivatives, Comp. Jn1., 7, pp155-62, 1964.

34. Nelder J.A., Mead R., A Simplex Method for Function Minimization, Comp. Jnl., 7, pp308-13, 1965. 
35. Wilkinson J. H., Solution of Real and Complex Systems of . Linear Iquations, Num. Math., 8, pp217-234, 1966.

36. Wilkinson J.'II., Three Contributions to Handbook Sexies Linear Aleobra, Num. Math., 4, pp354-376, 1962.

37. Tait K. E., Stability Properties of DiscreteContinuous Feedback Control Systems With Signal Dependent Sampling, Int. J. Control, Vol 8, No 6, pp591-620, 1968.

38. Brockett 2. E., The Status of Stability Theory ror Deterministic Systems, I.E. E. I., Trans. Aut. Contro1, AC-11, 596, 1966.

39. Reiss G. G., Inversion of The Vandermonde Matrix, I.E.E.I., $\mathrm{AC}-12, \mathrm{pp} 793,1967$.

40. Benny L.B., Mathematics for Students of Engineering: and Applied Science, Oxford Univ. Press, 1966.

41. Box M. J., Davies D., Swann W.H., Non-Iinear Optimization Techniques, I.C.I Monograph No 5 , Oliver and Boyd, 1969. 
42. Powell M.J.D., A Method for Non-linear Constraints in Minimization Problems, Optimation Symposium, Acadenic Press, 1968.

43. Lefshetz S., Stability of Non-linear Control Systems, Academic Press, New York, 1965.

44. Davies H.In, Introduction to Non-1inear Differential Eqns. \& Integral Eqns., (1962), Dover Pub. Inc.

45. Bream R.C., M Sc. Thesis, Optimal Lyapunov Functions, Loughborough University, 1970.

46. Nelder J.A \& Mead N., A Simplex Method for Function Minimization, Computer Journal, Vol 7, No.4, Jan 1965.

47. Davidson E.J.\& Kurak D.M, A Computational Method for Determining Quadratic Lyapunov Functions for Non-linear Systems, Automatica, Vo1 7, pp627-636, 1971.

48. Weissenberger S., Stability Analysis of Relay Control Systems via the Direct Method of Lyapunov, NASA, CR. 320, 1965.

49. Lapidus L \& Berger A, Stability of High Dimensional Nonlinear Systems using Krasovski's Theorem, A.I.CH.E. Journal 15, 1969, pp171-173.

50. Geiss G.R, Estimation of the DOA, Grunmman Res. Dept., Report RN 3167, Betgpase, NY, 1966.

51. Geiss, Coh en and Rothschild : Development of an Algorithm for NL Stability Analysis of an Orbiting Astronomical Abs. Control System, Grunmman R.D, Dep RE-307 1967.

52. Szego G.P, On the Numerical Construction of Lyapunov Functions, IFAC, No.20, pro 3-7, June 1969.

53. Smith R.A,/Mournal Diff. Eqns., Vo1 3, 2, pp208-217, 1966. 
54. Fletcher R., Function Minimization Without Calculating Derivatives - A Review, Comp. Jnl., $8, \operatorname{pp} 33-41,1965$.

55. Box M. J., A New liethod of Constrained Optimization and a Comparison With Other Methods, Comp. JnI, 8, pp42-52, 1965.

56. Box M. J., A Comparison of Several Current Optimization lethods, and the use of Transformations in Constrained Problems, Comp. Jn1., 9, pp67-77, 1966.

57. Julich P. M., On Estimating the Region of Asymptotic Stability Using Lyapunov's Method, I.E.E.E., AC-12, pp787-788, 1967.

53. Muddle R. P., Determination of Stability Boundaries Using Lyapunov Functions and Numerical Optimization, R.A.E. Report No. $67148,1967$.

59. Fiacco A. V. \& MicCormick G.'P., The Sequential Unconstrained Minimization Technique for Non-Linear Programming, a Primal-Dual Method, Mange. Sci., 10, $\operatorname{pp} 360,1964$.

60. Fletcher R. \& Powell M. J., A Rapidly Convergent Descent Method For Minimization, Compt. Jnl., 6, pp163-68, 1963.

61. Wilson F., The Structure of the Level Surfaces of a Lyapunov Function, Journal Diff. Eqns., 3, pp323-329, 1967 .

62. Miele A. - Coggins G. M., On the Method of Multipliers for Math. Programming Problems, JoTA, 10, No.1, 1972, pp1-32.

63. Fletcher R. \& Lill S., Proceed. of Non-linear Programming Symp., Madison, 1970. 
64. Fletcher R., Certification of Algorithm 251 Function Minimization, Comm. ACM, 9, pp686, 1966.

65. Haarhoff P. C. \& Buys J. D., A New lethod for the Optimization of a Non-Jinear Function Subject to Non-Linear Constraints, Conp. Jn1., Vo1 13, 2, pp173, 1970

66. Weissenberger S., Piecewise-Quadratic and PieceriseLinear Lyapunov Functions For Discontinuous Systems, Int. Jn1. Contro1, 10, No.2, pp171-180, 1969.

67. Kalman R. E., Lyapunov Functions for the Problem of Lure' in Autonatic Contro1, Vo1.. 49, 2, pp201-205, 1963.

68. Anosov D. V., Stability of the Equilibrium Positions in Relay Systems, Autmn. Remote Control, Vo1. 20, 2, pp130-143, 1959.

69. Alimov Y. I., On The Applications of Lyapunov's Direct Nethod To Differential Equations With Ambiguous Rigit Sides, Aut. Rem. Cortrol, Vol. 22, No. 7, pp713-725.

70. Rosenbrock H. H., Some Conditions for the Stability of Nonlinear Time-dependent Differential Equations, Jn1. SIAif Control, 2, pp171, 1964.

71. Weissemberger S., Desien for Stability Region Maxinization, I.E.E.E., AC-14, pp314-315, 1969.

72. Walker J.A. \& MCClamroch N. H.; Finite Regions of Attraction For The Problem of Lure', Int. J. Control, Vol. 6, No.4, pp331-336, 1967 . 


\section{Appendix 1.}

The following definition is important in defining the closed surfaces or contours of a continuous function $V(x)$ in some region $R(h)$.

Def. A1.1

The level surface $V(\underline{x})=c$ (the set of points satisfying $\mathrm{V} \rightleftharpoons \mathrm{c}=$ constant) is closed in $\mathrm{R}(\mathrm{h})$ if any continuous line from the origin meets this surface in at least one point.

Theorem A1.1

If $V(x)$ is a p.d. (n.d.) function in $R(h)$ then $\exists$ a $c$, such that for $0<c<c_{1}\left(c_{1}<c<0\right)$ the level surfaces $V=c$ are closed.

Theonem A1.2 (Barbashin (18))

If $V(x)$ is p.d. (n.d.) and radially unbounded i.e. $\lim V(\underline{x})=\infty(-\infty)$ $\|x\| \rightarrow \infty$

Its level surfaces are closed in $\mathrm{E}^{\mathrm{n}}$. 


\section{Appendix 2}

\section{Solving a Set of Linear Equations}

Since we are interested only in the 1 th stage of the calculation of the $v_{i j}$ we omit the $i$ subscript. The set of linear equations 4.1 .6 gives $(n=N$ in $C 3)$

$$
\begin{aligned}
v_{1} a_{1}+v_{2} b_{1} & =c_{1} \\
v_{2} a_{2}+v_{3} b_{2} & =c_{2} \\
\cdot & \cdot \\
\cdot & \cdot \\
v_{n-1} a_{n-1}+v_{n} b_{n-1} & =c_{n-1} \\
v_{1} b_{n} \cdot \quad \cdot \quad+v_{n} a_{n} & =c_{n}
\end{aligned}
$$

or $\quad A \underline{V}=\underline{C}$

Consider the following two algortthms:

a) starting with 1st equation express $v_{j}$ in terms of $v_{1}$ up to the $n-1$ th equation; substition into the last equation then gives

$$
\begin{array}{r}
v_{1}\left(1-(-1)^{n} \prod_{1=1}^{n} a_{1} a_{1}\right)=\left(\frac{c_{n}}{b_{n}}-\frac{a_{n} c_{n-1}}{b_{n} b_{n-1}}\right. \\
\left.\quad \frac{a_{n} a_{n-1} c_{n-2}}{b_{n} b_{n-1} b_{n-2}} \cdot \cdot \cdot \cdot \cdot \cdot \cdot a_{2} c_{1}\right) \\
(-1)^{n+1} \frac{a_{n} a_{n-1} \cdot \cdot b_{2}^{b_{1}}}{b_{n} \cdot \cdot \cdot b^{2}}
\end{array}
$$


If $b_{i} \neq 0 \& 1-(-1)^{n} z \neq 0$ back substition gives

$$
v_{j}=\frac{1}{b-1}\left(c_{j-1}-a_{j-1} v_{j-\pi}\right), j=2, N
$$

where

$$
z=\prod^{n} \frac{a_{j}}{b_{j}}
$$

b) starting with the last equation in $\mathrm{A} 2 \mathrm{C}$

express $V_{j}$ in terms of $v_{1}$ down to the 2nd equation substition into the 1st then gives

$$
\begin{aligned}
v_{1}\left(1-\frac{(-1)^{n}}{2}\right)= & \left(\frac{c_{1}}{a_{1}}-\frac{b_{1} c_{2}}{a_{1} a_{2}}+\frac{b_{1} b_{2} c_{3}}{a_{1} a_{2} a_{3}}\right. \\
& \cdots(-1)^{n+1} b_{1} \cdot b_{n-1} c_{n} \\
& \frac{a_{1} a_{2} \cdot a_{n-1} a_{n}}{a}
\end{aligned}
$$

Provided $a_{i} \neq 0 \quad 1-\frac{(-1)^{n}}{2} \neq 0$ back substition gives,

$$
v_{n-j+1}=\frac{1}{a_{n-j}}\left(c_{n-j}-b_{n-j} v_{n-j+2}\right) .
$$

$$
\mathrm{v}_{\mathrm{N}+1 !}=\mathrm{v}_{1}
$$

Thus provided $a(A)=\left(\prod^{\pi} b_{i}\right)(z-1) \neq 0$ and with round off errors in view, choose algorithm
(a) if $|z| \leq 1$
and
(b) if $|a|>1$ 


\section{Appendix 3}

A Crude R.A.S.

With the assumptions on $(x)$ in 2.1 , the bound

2.1 .19 holds in some region $\mathrm{R}(\mathrm{h})$ for which $\beta>0$ 1.e.

$$
\|g(x)\| \leq \frac{n}{2 \mu}\|x\|
$$

It is easily seen that A 3.5 is satisfied for all solutions of 2.1 .2 with

$$
\|\underline{x}(0)\|<\mathrm{h} / \sqrt{\mu} \quad \text { A } 3.6
$$

where $h=\min \|\underline{x}\|$ for $\underline{x}$ satisfying $A 3.5$

For then $\|\underline{x}(t)\|<h$ by 2.11.21 which implies:

A 3.5 and hence $\beta>0$.

When $\underline{u}=0$ and $G(\underline{x}, t)=G(\underline{x})$ in 2.1 .1 , a possibly

sharper estimate can be obtained if $\lim G(x)=0$.

The crude R.A.S. is given by A. 3.6 with

$$
\mathrm{h}=\min _{\underline{x}}\|\underline{x}\|
$$

for $\underline{x}$ satisfying $\|G(\underline{x})\|=n / 2 \sqrt{\mu}$

The Minimizing $D$ (Bauer (22))

The diagonal matrix $D$ giving the bound in 2.3 .11 is determined as follows.

Let

$$
M=\left|s^{*} s\right|\left|\left(s^{*} s\right)^{-1}\right|
$$

and for this non-negative matrix let $e^{T} Y_{1}$ and $\underline{X}_{t}$ e be left and right eigenvectors (Perron) of $M$ corresponding to the largest positive eigenvalue of $M$, 
where $\underline{e}=(1,1, \ldots, 1)$ and $y_{1}$ and $x_{1}$ are diagonal matrices. Then

$$
\mathrm{D}=\mathrm{Y}_{1}^{\frac{1}{2}} \mathrm{X}_{1}{ }^{-\frac{1}{2}}
$$

\section{A Minimization Algorithm}

Lat $p_{i j}$ and $q_{i, j}$ be the $i, j$ tin elements of $P$ and $Q$ where $Q$ is any p.a.s. matrix with $m(=n(n+t) / 2)$ elements. The optimization problems are to minimize

$$
\begin{aligned}
& \text { (a) } s\left(P^{-1} Q\right)=M\left(P^{-1} Q\right)-m\left(P^{-1} Q\right) \\
& \text { (b) } \mu(P) \\
& \text { (c) }-\eta \\
& \text { (d) }-\eta^{2} / \mu
\end{aligned}
$$

over the $\left(m-1_{i}\right)$ dimensional space of elements $q_{i j}$ subject to

$$
Q>0 \text { (positive definite) }
$$

where $A^{\mathrm{T}} \mathrm{P}+\mathrm{PA}=-Q$

(Note one element of $Q$ can be aribitrarily chosen)

The non-linear optimization algorithms of Rosenbrock (32) or Powell (33) are well suited to these problems and standard subroutines are available. (See A4). These routines require an auxiliary routine to evaluate the objective function and in this case the main steps were:

$$
\text { (1) from } I \text {, a lower triangular matrix input }
$$
to the routine, calculate $Q$ via

$$
\mathrm{Q}=\mathrm{I}^{\mathrm{T}} \mathrm{I}+\epsilon \mathrm{I}
$$

which is p.d. for $\epsilon>0$; 
(2) Solve A 3.16 for $\mathrm{P}$ by the direct method (20) 1.e. solve

$$
=-q \quad \text { A } 3.18
$$

where $\mathrm{p}=\left(\mathrm{p}_{11}, \mathrm{p}_{12}, \mathrm{p}_{22}, \mathrm{p}_{13}, \mathrm{p}_{23}, \ldots \mathrm{p}_{\mathrm{nn}}\right)$

$$
g=\left(q_{11}, q_{12}, q_{22}, \ldots \ldots \ldots, a_{n n}\right)
$$

and $B$ the $m x$ matrix of coefficients (See Barnett (20));

(3) compute $R$ and $R^{-1}$ where $P=R^{T}$ and $R$ a lower triangular matxix;

(4) compute $M\left(P^{-1} Q\right)=M(T)$ and $\Pi=m(T)$ where $T=R^{-T} R^{-1} \quad\left(T \sim P^{-1} Q\right)$;

(5) For (b) and ( $d$ ) compute $m(P)$ and $M(P)$. The introduction of $I$ avoids the $n$ implied constraints of A 3.15 and the minization is thus over the $\mathrm{m}-1$ elements $\left(I_{i j}, I=2 ; n, j=1,2 . .1\right.$ ) of $I\left(I_{11}=1.0 \mathrm{say}\right)$ (In practice A 3.18 was solved by a Crout iterative method (35) and the eigervalues of T by the method of Householder (36). The choice of $\epsilon=10^{-10}$ was sufficient in A 3.17 . 


\section{Appendix 4}

The optimization methods used in this thesis concern the programming problem

$$
\min F(x) \quad 44.1
$$

subject to (a) an equality constraint $e(\underline{x})=0$

or (b) an inequality constraint $c(x)=0$

The class of methods used to solve A4.1 depends on the form of $F$ and the constraints. Fletcher (54) and Box (56) give good accounts. The methods of Rosenbrock (32), Powe11(33) and Nelder and Mead (34) are well known and standard packages were used written in Fortran IV. We only mention that Rosenbrock deals with the constraint, $c \leq 0$, by introducing an implicit variable $x_{n+1}=c$ which is deemed feasible if

$$
z\left(1-10^{-4}\right) \leq x_{n+1} \leq z 10^{-4}
$$

where $z$ is a lower bound for c. Should this not be satisfied but. $x_{n+1}$ lies in one of the boundary zones $z 10^{-4}<x_{n+1} \leqslant 0$ or $z \leq x_{n+1}<z\left(1-10^{-4}\right)$ a special penalty function is used otherwise the value is termed a failure. Box's Complex Method For Constraint (b)

A method modifying the Simplex method, it consists of forming a complex - a set of $K(\geqslant n+1)$ independent points $\underline{p}_{j}(j=1, \ldots, K)$, at which $F$ is evaluated. Initially, assume all points are feasible i.e. $c\left(\mathfrak{p}_{j}\right) \leq 0$. Let $\mathfrak{p}_{h}$ correspond to $F_{h}$, the highest value of $F$ in the complex, and $\bar{p}_{h}$ the centroid of points $\mathrm{I}_{\mathrm{j}}$ excluding $\mathrm{p}_{\mathrm{h}}$. Then a stage consists of the following: with $\alpha=\alpha_{0}$ and

$$
\underline{p}=\bar{p}_{h}+\alpha\left(\bar{p}_{h}-\underline{p}_{h}\right)
$$

(an over-reflection of $p_{n}$ through the centroid) evaluate $F(p)$ and $(1)$ if $c(p)>0, \alpha$ is halved and the calculation repeated until $c(p) \leqslant 0$, then (2) if $F(p)<F_{h}$ is not satisfied $\alpha$ is 
again halved until it is. The final values of $\mathbb{D}$ and $F(p)$ then replace $p_{h}$ and $F_{h}$ and new values of $p_{h}$ and $F_{h}$ are found with new centroid

$$
\bar{p}_{h}(\text { new })=\bar{p}_{h}(o l d)+\left(p-p_{h}\right) /(K-1)
$$

This completes a stage. The calculation is renefted until arter I stages, $I \geq I_{\max }$ or $\left|F_{n}-F_{\text {lowest }}\right|<\epsilon \cdot\left(\operatorname{In} C 5, \alpha_{0}=1.3\right.$, $\left.K=2 n+1, \epsilon=10^{-4}\right)$.

\section{Penalty Function Updating}

With respect to the penalty function of Miele (62),

$$
W(\underline{x})=R(\underline{x}, \lambda)+K e^{2}(\underline{x})
$$

the following rules are used for the conjugate gradient(DFP) algorithm :

(1) Initially select $\lambda_{=} \lambda_{1}=0, K=K_{1}\left(K_{1}=20\right.$ say $)$

(2) With some initial $\underline{x}_{0}$ and $H=I$ perform $n$ iterations of DFP on $W(\underline{x})$. Let $\underline{x}^{*}$ be the final point.

(3) If $\left\|\mathbb{R}^{R}\left(\underline{x}^{*}, \lambda_{1}\right)\right\|<\epsilon$ and $\left|e\left(\underline{x}^{*}\right)\right|<\epsilon$ convergence is assumed, otherwise

(4) An updated $\lambda$ is chosen to minimize $\left\|\nabla R\left(\underline{x}^{*}, \lambda_{2}\right)\right\|^{2}$, of the form $\lambda_{2}=\lambda_{1}+2 \gamma_{e}$, which gives

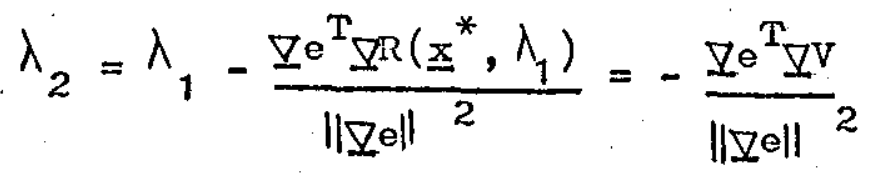

The value of $K$ is either kept constant or a criterion of reducing $\|\mathbb{R}\|$ and lel at the same rate is employed which gives for the updated $\mathrm{K}, \mathrm{K}_{2}$;

$$
\begin{aligned}
& K_{2}=\min \left(K_{0}, K_{1}\right) \text { if }|e| \leqslant\left\|\underline{R}\left(\underline{x}^{*}, \lambda_{2}\right)\right\| \\
& K_{2}=\max \left(K_{0}, \beta K_{1}\right) \text { if }|e|>\left\|\nabla R\left(\underline{x}^{*}, \lambda_{2}\right)\right\|
\end{aligned}
$$

where $\beta \succeq 1$ and $K_{0}=\left|\lambda_{2} / e\right|$ where the latter makes the order of 
the term $\mathrm{Ke}^{2}$ the same as that of $\mathrm{R}$. Step (2) is now repeated. Fletcher Powell Nethod (DFP)

When derivatives of A5.1 are available the algorithm determines a sequence of iterates $\underline{x}_{i}$ and directions $\underline{s}_{i}$ as follows : with some initial p.d.s. $H, H=I$ say, and initial $\underline{x}_{0}$,

$$
\underline{x}_{i+1}=\underline{x}_{i}+\lambda \underline{s}_{i}=\underline{x}_{i}+\underline{z}_{i}
$$

where $\underline{s}_{i}=-H_{i} \underline{s}_{i}, \quad g_{i}=\nabla F\left(\underline{x}_{i}\right)$

and $\lambda$ determines the minimum of $F$ along a line

$$
\left.\frac{\partial F}{\partial \alpha}\left(\underline{x}_{i}+\alpha \underline{s}_{i}\right)\right|_{\alpha=\lambda}=0 .
$$

Here $H_{i}$ is updated as

$$
\mathrm{H}_{i+1}=\mathrm{H}_{i}+\frac{\underline{z}_{i}^{\mathrm{T}} \underline{z}_{i}}{\underline{z}_{i}^{\mathrm{T}} \underline{u}_{i}}-\frac{\mathrm{H}_{i} \underline{u}_{i} \underline{u}_{i}^{\mathrm{T}_{\mathrm{H}}}}{\underline{u}_{i}^{\mathrm{T}} \underline{H}_{i}}
$$

where $\underline{u}_{i}=\underline{g}_{i+1}-\underline{g}_{i}$.

A converted Algol program of Fletcher (64) was used where cubic interpolation is advocated to determine the min. along a Iine. The initial step, $\lambda=s_{1}$, along the latter was restricted to

$$
s_{1}=\frac{0.1\left\|\underline{x}_{0}\right\| s_{i}}{\left\|\underline{s}_{i}\right\|}
$$

for the problem in Chapter 5. 


\section{Appendix 5}

\section{The Complex Optimal Quadratic Algorithm}

The program, designated 1459 , is written in Fortran 4 and flow diagrams are given in Figures 5.1 and 5.2 . Access to the ICL library of scientific subroutines is needed to run the program (TPQRVS, FPDIRHESSF, FPQRHESSE, FPBACK, F4ACSL and F4ROOT1 are from it). The instructions are intended for guidance in running the program.

\section{Input}

The sequence of input variables are as follows:

1) $N$ and LOADQ are set in the Master segment; $n$ being stored in $N$ and LOADQ being of logical value. TRUE. or . FALSE. .

2) The initial complex matrix $\mathrm{D}$ is input and stored in $\operatorname{array} \operatorname{DEL}(i, j)(j=1,2 n, i=1, n)$

3) The elements of $A, a_{i j}$, are input in array $A(I)$ such that $A(k)$ contains $a_{i j}, k=n(j-1)+i$. (In the Rao system in the listing, $A(k)$ have been calculated in the program for accuracy).

The r.h.s. of the system $\underline{f}(x)$ are set through subroutine $\operatorname{CALFX}(N, X, F)$ where $X$ and $F$ are one-dim, arrays containing $x_{i}$ and $f_{i}$. Unit vectors $y_{j}$ are stored in array $Y(i)$ such that the $i^{\text {th }}$ component of $y_{j}$ is contained in $Y(n(j-1)+j)$. They are calculated through a call to $\operatorname{SETUNVEC}(S, N V, N, Y, N X, P)$ where $s$ is the step in $\theta_{1}$ and $s / \cos \theta_{2}$ the step in $\theta_{2}$ for unit vectors calculated via polar co-ordinates 4.5 .1 ; NV is the number of unit vectors calculated; $N X=N(N+1) / 2$, and the array $P(i)$ contains the initial $P$ such that $p_{i j}(j=1, n, i=1, j)=$ $P(i+j(j-1) / 2)$. If the 'box' method is used $S$ is imaterial and N1, the number of mesh points along a half-edge of the box, is set in SETUNVEC ( $N 1$ is set after statement $61 . \mathrm{S}$ is set after statement 100 in the master segment). 


\section{Output}

After some checking output the following sequence is observed:

1) The elements of initial $P$ and $Q$ are output in the order that the $p_{i j}$ are read.

2) Aftox cach cvaluation of $\dot{V}_{M}(1)$ the values of $y_{\text {max }}$, $\dot{V}_{M}(1)$ and 1 are output in order, where $y_{\max }$ gives $\dot{V}_{M}(1)$.

3) The final $1, I_{2}$, is output (called E) followed by the volume of $\underline{x}^{T} P_{x}<1_{2}$.

4) Output from the setting up of the initial feasible complex - rows containing $\rho(v \circ 1), \dot{V}\left(p_{j}\right)$ and $s$ (initially $\left.2 \max \left|t_{i}\right|\right)$. If no feasible complex is found the words

'IFEASIBLE SIMPLEX'

are output.

5) The complex points $p_{j}(j=1,2 n+1)$ are output.

6) After every 10 evaluations of $\dot{V}_{M}(L), y_{\text {nax }}, p_{i j}(j=1, n$, $i=1, j), \dot{v}_{M}(L)$ and $-p$ are output. Then $t_{i}(i=1, N X)$ are output on a new line (elements of $L$ ). The co-efficients of $y_{\max }$ follow the word 'MAXVDOT'. 
MASTFR $1455^{\circ}$

I.NGTCAL 10400

COONDIEX SS $(4,4), S U N$

DI YENSIOM DEI $(6,12)$

DIMEIS IIIN T $(190), A(16), X A(16), R E I N T(5), X(16), D(10)$

DTMENSTONN V $(4000)$

DTMENSIOHE INT $(G)$.

DIMENSION G(94), H(14)

$A A(16), E X(4), E Y(4), I T S(4), A A A(16)$

$N=$ ?

$N=3$

$N X=(N *(N+1)) / 2$

$N K=2 * N X$

$N S Q=N * N$

RFAD $(1, q)((N F L(I, J), T=1, N X), J=1, N K)$

IOANO=. TRUE.

IOAND $=$. CAISF.

RFAn $(1.9)(A(1), I=1, N S Q)$

1 FORYAT (6F1?.5)

$7>=\cos (473)$

$71=59 N(478)$

$A A(1), A A(6), A A(7)=0$.

$A A(C)=1$

$A A(j)=43.06 * 0.0 S(.956)-84.99 * 1.98 * 72$

$A A(3)=0 * 21 * 71$

$A A(3)=-A A(3)$

$A A(5)=080 ? * 72 * 22+1.04 * 21 * 21$

$A A(5)=-A A(5)$

$A A(R)=-34.09 * 79$

$A A(0)=-621$

DO \& $3 \quad I=1$. NSO

$83-A(T)=A A(T)$

IF(INAAO) GO TO 69

c

DO in $1=9, \mathrm{NSO}$

$10 \quad A A(1)=A(1)$

$F(N-2) 0,0.49$

$71=(A(1)+A(4)) / 2$.

$72=A(9)+A(6)-A(2) * A(3)$

$n=71 * 21-72$.

$D 1=S D R T(A R S(D))$

$I F(D) 0.52 .52$

$F \times(1) . E \times(2)=79$

$F Y(1)=-n 1$

$F Y(3)=109$

60 In 53

$52 F Y(9), F v(2)=1)$

$F \times(9)=7.1-01$

$F X(7)=71+n 1$

53 CALI FPORVS (N.AA(1),AAA(1),FX(1),FY(1),T(1))

GO TO 48

4Q. CONTINIJF

CAII FPOTRHESSF(N,AAC1), INT (1))

IVS $=$ ?

CALI FPORHESSF (N,AA(1), TTS(1), FX(1),FY(1),AAA(1),IVS)

c

FIGFNVALUFS ARE $E X+E Y$

CAL! FPORVS(N,AA(1), AAA(1),EX(9),EY(1), T(1))

CALI FPRACK (N.AA(1),AAA(1),EY(1), INT(1))

48 CONTINUIE

WRITF(?.44) (TAAA $(1+N *(J-1)), J=1, N), I=1, N)$

c

44 FOR:IAT ( $3 F 92.5)$ 
$I=1$

6 IF(ARS(EY (J)) -1.0E-10)0.0.2

no $3 \quad 1=1, N$

$K=I+N *(I-1)$

$3 s S(i, J)=\operatorname{SPP} \times(A A A(K), 0.0)$

$J=l+1$

an $\operatorname{ton} 5$

$2 D 0 \quad \& \quad I=9, N$

$K=I+N *(1-1)$

$S S(I, J)=C M P(X(A A A(K), A A A(K+N))$

$4 S S(j, J+1)=C H D L X(A A A(K),=A A A(K+N))$

$J=J+?$

C

5 IE $(.1-N ; 6,6.0$

DO $\angle 9 \mathrm{~J}=9, \mathrm{~N}$

FIND INIT VECTORS

$S M=0.0$

DO $4 ? I=1, N$

$A M D=C A B S(S S(1, J))$

$42 . S M=C M+A M D * A M D$

SUN $=$ CMDIX X SNRT (SM), O.O)

$D O \& 1 \quad I=1, N$

$41 S S(1, J)=S S(1, J) / S U N$

WRITE(?.43)(CS $(I, J), J \equiv 1, N), I=1, N)$

43 FORMAT (3(2E1?.5))

C

FIND $P=S * S$, REUSF AAA FOR STORAGE

DO $7 . J=1 . N$

$R E-U S E$ AA TO SOLVE AAA $S * S=I$

DO $7 \quad 1=0, N$

$S U N=(0.0,0.0)$

DO $\& \quad K=1, N$

$8 \quad S U N=S U N+\operatorname{CONJG(SS}(J, K), 2+S S(I, K)$

$K K=i+N *(J-1)$

WRITE(2.51) SIJN

51. FOR

$7 A A A(K K)=R E A L(S U N)$

C. IN $\times \overline{1}$

DO $11 \mathrm{~K}=1$.NSR

$11 \quad A A(\underline{x})=00$

DO $1>I=1, N$

12. $A A(T+N(Y-1))=1.0$

CALI FLACSL (AAA,AA,N,NSQ,NSQ,IN,X,O,ID,IT,XA,T, REINT)

DO $13 \quad J=1, N$

no $13, i=1, J$

$K=1+(J *(1-1)) / 2$

13. $P(K i=X(i+N *(1-1))$

GO $T 0$, 6 ?

$61 N S Q T=N S O+N S Q$

CALI SETA (N,NSQ,NSQ2,T,A)

C READ Q MATRIX RY ROWS

C DIM $O E Q=N+N$

$R E A \cap B(1, T)(X A(1), 1=1, N S Q)$

$I N=9$

CALI FGACSLCT,XA,NSQ,NSQ2,NSQ, IN,AA,D, ID,II,Y,AAA,A)

$K=0$

DO $63, I=9, N$

DO $43, J=1,1$

$K=k+1$

$63 P(K)=-A A(A+(1-1)+J)$

WRITF (2.05)

65. FOR IAT ( $\cap \cap X, 1$ GHINITIAI Q MATRIX)

WRITF(2.,1) $($ IXA $(N *(I-1)+J), J=1,1), 1=1, N)$ 
62 RONTINUE

WR!TF(?.45)

45 FOR MAT (IX.OHTNITIALD)

WRIYF(?: 44$)(R(K), K=1, N X)$

$X(1)=\operatorname{SART}(P(1))$

no $14 \quad J=?, N$

$k=(1 *(J-1)) / ?+1$

$14 x(K)=D(K) / X(1)$

no is $I=2, N$

$k q=(1 *(\mathrm{r}+1)) / ?$

S $(1)=P(K T)$

$k 2=(1 *(1-1)) / 2$

no $5 \quad l=1,1-1$

15 SUM $=S U M-X(K 2+L)+X(K 2+L)$

$x(K T)=\operatorname{SORT}(\mathrm{S}(\mathrm{I} M)$

nก $16 \mathrm{~J}=1+1 . \mathrm{N}$

$K=(1 *(J-1)) /$ ?

$S U M=D(I+K)$

no $1>1=1 \cdot 1-1$

17 SUM $=S U H-X(L+K 2)+X(L+K)$

$16 \times(I+K)=511 M / X(K 1)$

WRITF $(2.47)(X(1), I=1, N X)$

47 FORAAT $(9 X .9$ HINITIAL $\times .3 E 12.5)$

C

100 CONTINUE

START INITIAL VDOT CONTACT

$F R 1=2$

$F R 9=3$

$S=.1$

$s=$ ?

CALI SETINVEC $(S, N V, N, Y, N X, P)$

$i T=0$

$F 1=1: 05-5$

$F 1=1 . O F-10$

$F 1=01$

$N N V=N \backsim N V$

SMAX $=10$ " 0

$s=\cdot 1$

101 CONTTNIIE

CALI VMAXI (N,NV,Y,NNV,X,NX,TI,EI,SMI)

IF TSMYI 20.0 .0

$F 1=1 * F 1$

$I F(E 9-1,0 E-10) 0,109,109$

WRITF $(3.21)$

21 FORMAT (AX.14HSMALL VOL STOP)

$7=1 \div 10$.

$S T O D$

$20 F ?=F 1+S$

CAI! VMAXI (N,NV,Y,NNV,X,NX, I1,EZ,SM2)

If isM2i $0.23,23$

$F q=F$ ?

$S M 9=S M 2$

If $(S-\operatorname{siAX}) 75,25,0$

WRITF $(2,24)$ E1

24 FORMAT i9X .16HVOOT NOT REACHED.E92.5)

$S T O D^{\circ}$

$25 s=2 * s$

an $r$ in $2 n$

23 CONTINUF

$S=S i ?$.

$27 \quad F 3=F 1+s$

CALI VMAXI (N,NV,Y,NNV,X,NX, II,EZ,SMB

SEST?. 
IF $\operatorname{ssm} 3 i 0,28,28$

$S M 9=S M 3$

$F 1=F 3$

an tก 20

28 SM? $=543$

F2 $=53$

20 $F=S M 1 / E 9$

$I F(J * S I E 2-F R 1) 0.27,27$

WRITF (?.66)F?

46 FORYAT( $X .9$ OHVALIIE OF E.F12.5)

$N W=1000$

NWa? 000

$F=\operatorname{SOR} T(F 1)$

$Y(N w)=H N$

$Y(N \cdot i-1)=F$

$V(N, 1-2)=N$

$i v=n$

CAII CAIF (NX,X,F,M,IV,XM,Y,NW)

$X A(1)=F$

WRITF (?.1)E

$F F=1$ I

no $81 \quad l=1 . N x$

$89 T(1 T, X(T)=X(1) * E E$

STR $=.9$

$V(N-1-1)=1$

$S T P=0$.

DO $R>i=1, N X$

82. STP $=A H A \times 1(S T P, A B S(X(1)))$

$S T D=1 \div S T P$

C CAL DTS OF NON REGUIAR SIMPLEX $K=N X+1$

$I V=1$

DO $84 \mathrm{~J}=9$, NK

HSESTP

95 NFG $=0$

93 NFG $=N E G+1$ nO $86 \quad 1=1, N X$

$86 G(I)=X(i)+D F L(I, J) * H S$

87 CAL CAIF (NX,G,F,M,IV,XM, Y,NW)

$X A(1+1)=F$

WRITF(2.1) F,XM,HS

IF(XM) 85,85,0

I. $(N F G-T) 0,0,94$

HS $=$ in $H$

GO in 9 i

9.4 HSI-HS/?

IF(STP* O1-HS) $95,92,02$

$85.00 .891=1 . N X$

$T(K)=G(Y)$

$89 K=K+9$

84. CONTINUF

GO TO BR

92 CONTINUF

WR ITF $(2.90)$

90 FORMAT ( $1 \times .18 H I N F E A S I B L F$ SIMPLEX)

STOD

$88 K 2=\bar{K} 2+N \dot{X}$

$N K=N K+1$

WR ITF (?:1) $(H(I), I=1, N X)$

WRITF $(2.1)(T(I), I=1, N X * N X)$

$Y(N$ ij- 1$)=1.0$

$M F=500$ 
$M F=300$

$M F=1000$

$F D S=A B S(X A(1))+1.0 \mathrm{E}=3$

$F P S=1.0=-4$

FDS $=A B S(X A(1))+1.0 E-4$

$N K=j * N X+1$

$N P=(N K+T) \approx N X$

CALI COMPSIM XX,F,EPS,T,XA,NX,ND,MF,Y,NW,NK)

Go in 9 ino

$S T O P$

FND

SUBROUTINE AMATV $(X, N X, B, N)$

$D I M F N S \operatorname{InN} X(N X), B(N)$

C

ROUTINE MULTIPLY XB-X UPPER TRIANGULA

DO $>1=9 . \mathrm{N}$

RY COLS. STORE RFSULT IN B - A VECTOZ

$S U M=0.0$

no $1: J=i, N$

$K=1+(J *(J-1) / 2)$

1. $s(M M=S U M+X(K)+B(J)$

2. $B C T$ I $=S U M$

RFTIIRN

END

SUBROUTTNE CATFX(N,X,F)

AIMENSION $X(N), F(N)$

$E=x(3)+1.181665534$

$D=X^{\prime}(9)+4779929590$

$A=\sin (0 ;$

$R=C \cap S(D)$

$F(9) \times x(3)$

$F(2)=28 \% 61-84.09 * E * A+21.53 * \operatorname{SIN}(2, * D)-X(2) *($

$11.04 * A * A+0.8 \cap 2 * B * B)$

$F(3)=.36-.621 * F+.421 * B$

RETIIRN

END 
SURROUTTNE COMPSIM(X,F,FPS,D,FP,N,NP,MF,V,NW,NK)

DIMFNSION $X(N), P(N P), F P(N K)$

DIMENSIIN $Y(N W)$.

DIMENSION XS $(10)$

$1 C=10$

$N N N=Y(N W-2)$

$A D O A=5$

$A P O=1.3$

$N N=N * N K$

$N 1=N K$

IV $=$ in

$I S H=0$

$M=1$

c.

SORT TH(TGHEST) AND II (OWFST)

35 IFCEP(1)-FP(2) $0,0.5$

$I H=2$

11.1

60 TO $\mathrm{K}$

5. $I H=1$

$11=?$

$6 \mathrm{DO} 71=3 . \mathrm{N1}$

IECDP(I)-FP(IH)) 0.0.8

IF(FD(I)-FP(IL) $0,7,7$

$11=i$

an To?

8. $T H=1$

7 CONTTNIIF

C ISHEO RFELFCTION,FIND CENTROID OF SUBSIMPIEX

IE $(T S H) \cap 0,30$

$X N=N K-1$

$50 \times 1=N N$

$D O Q . \quad I=1 . N$

$K=1$

$s=0$

$D O-10 \mathrm{~J}=1 . \mathrm{NI}$

IF (I-IHIO.10.0

$s=s+D(k i$

$10 \quad K=K+N$

$K 1=K 1+1$

9. $P(K i)=S / X N$

WRITF (2.71) $(P(N N+1), I=1 . N)$

71 FOR MAT $(\bar{T} X, 6 E 12.5)$

$155 K=(Y H-1) * N+1$

$Y(N(j-3)=1 C$

67 IV $=1$

$A D=\triangle D O$

$62 A P q=A P+1$.

$K O=N N+1$

Dn is $\quad I=1, N$

$x(1)=A P I * P(K 0)-A P * P(K)$

$K=k+1$

16. $K 0=K 0+1$

$K=K-N$

CALI CAIF (N,X,F,M,IV,XM, Y,NU)

$M=M+1$

If (IV)0.61.0

$I F(X M) 69.61,0$

$A D=A D / 2$

GO TO G?

61 IF $\left(5-F D^{\circ}(1 H)\right) 64.0 .0$

$A D=\triangle D /$ ? 
$i v=n$

G0 Tत 6?

64 CONTTNUE

DO $65 \quad 1=1, N$

$D(k)=x(i)$

$65 k=K+1$

$K=K-N$

FP( IH)

I $S H=1$

on 1035

$30 \times N=N K=1$

IF( $(E P(T H)$ OFP(IL)) NEDS $100,100,0$

IF $(M-M E-1) 0,100,100$

C NEU CFNTROIO

$K 1=(1 H-1) * N+1$

$K>=N N+1$

no $66 \quad i=1, N$

$P(K)=P(K))+(X(I)-P(K I)) / X N$

$K 1=K 9+1$

$66 k \geqslant=k ?+1$

$r e q i c+1$

IF( $)(0-1 n) 155,0.0$

$$
I C=0
$$

Go $\operatorname{Tn}$ in

DO \& $I=1, N$

$81 \times S(I) \neq D(N N+T)$

I $=90$ :

CAII CAIF(N,XS,FC,II, TV,XMC,Y,NW)

WRITF(?.72) $(P(N N+1), I=1, N), F C, X M C$

72 FORMAT $(9 X, 1 H C, 3 E 92.5)$

WR TTF (?.73)IH.1L

73 FORMAT $(1 \times, 215)$

$K I=(T(-1) * N$

WRITF(2,?) $(P(K 1+I), I=1, N), F P(I L)$

2 FORMAT $(9 X, 3 H \times L ., 6 E 12,5)$

102 CONTIHUE

WR ITF $(?, 3)(X(1), 1=1, N), X M, F$

3 SORMAT $(1 X, 3 H \times R=, 6 E 92.5)$

$K 1 \cdot k ?=0$

no $104 . i=1, N N N$

$K 2=\times 1$

no ins $\quad I=I, N N N$

$K=1+K 2$

$X S(k)=0$

Do $106 \quad i=1,1$

$106 \times S(K)=x S(K)+x(K 1+L)+\times(K 2+L)$

$105 k 2=k 2+j$

$104 \times 1=x+1$

WRITF $(2,3)(X S(1), 1=1, N)$

(io in 155

$K 1=0$

DO OA $I=1, N K$

WRI TF $(2.71)(D(K 1+J), I=1, N), F P(1)$

$99 . K 9=\vec{K} 9+N$

100 COATTNUF

RFTIIRN

FND 


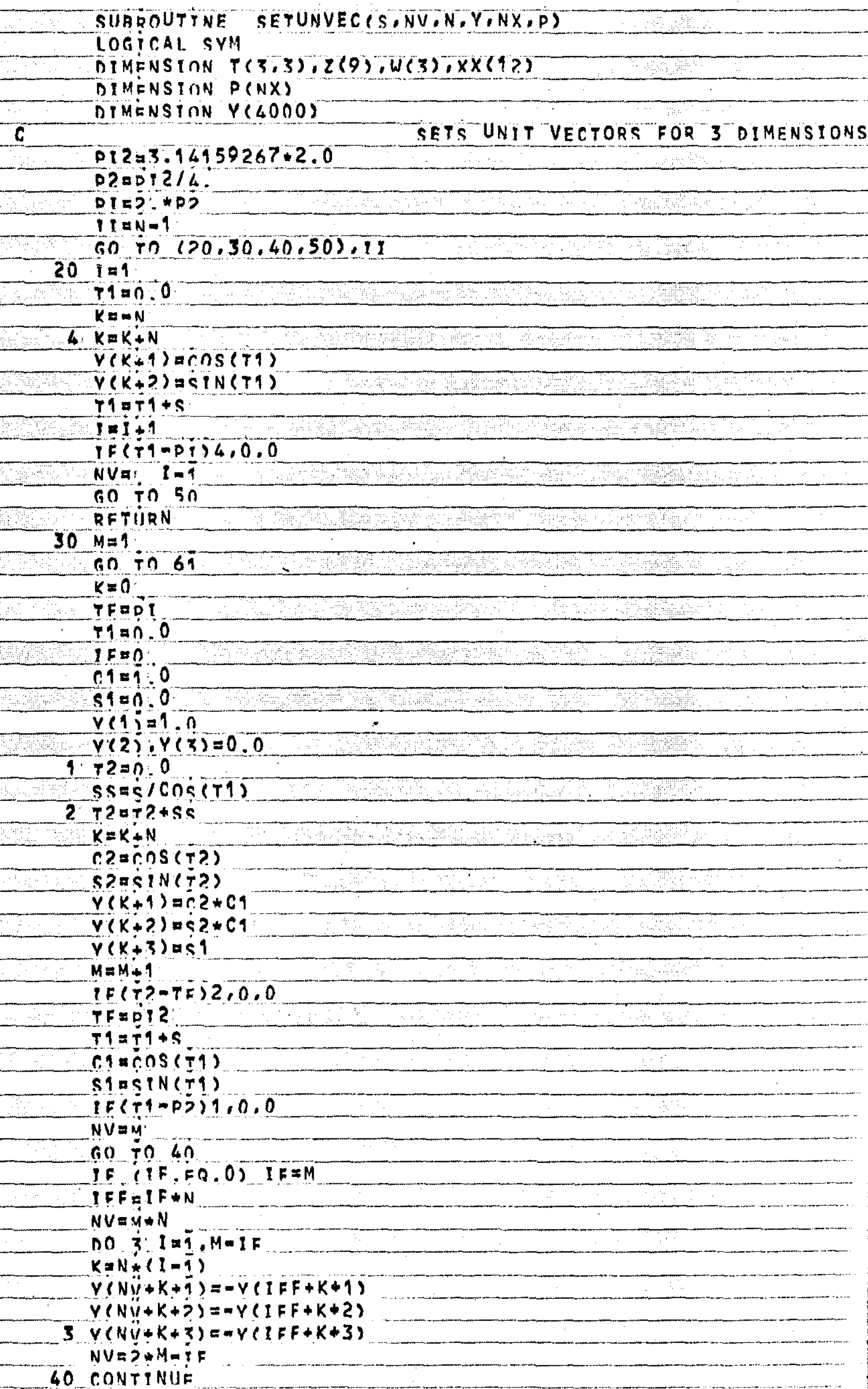


61. CONTTNUF

ND $=2000$

NPajo00

NP $=1500$

$N D=3000$

$N D=4000$

SYM TRUE.

SYM FAISE.

$N 1=15$

$N 1=5$

$N 9 \geq 8$

$N I=K$

$N 9=5$

$N 9=10$

$M 4=4 * N$

$M 2 \square N * N$

IV $S=0$

NEF 3

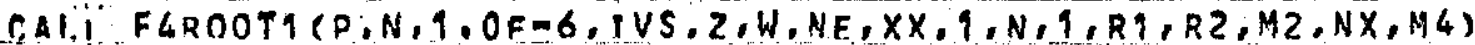

DO $62 \quad 4=1, N$

$D O$. $P ? \quad I=1, N$

$K=N+(N-i)+1$

$62 T(1,1)=?(K)$

BESORTIU(N)/W(1))

$A=\operatorname{SORT}(\mathrm{N}(N) / W(2))$

$X N=N 1$

N2?N3=N?

S.S $1=1 . \bar{T} \times \mathrm{N}$

$S ?=-1 \times N$

$S 3=\mathrm{B} / \times \mathrm{N}$

WRITF(2.51)(W(1),I:1,N),A,B,S

WRITE (2.52)N9,N2,N3

52 FORMAT $(T / 1 X, 415 /)$

C

RASE NF CYLNDR.

$K=0$

$x=1:$ :

no $73 \quad I=9,2$

DO $74, J=1, N 1+1$

$x J=i-1$

$y(k+1)=x$

$y(x+2)=x J \star s 2$

$v(k+3)=0$.

$74 K E K+N$

$73 x=9:$

DO $75 I=1,2 * N 1+1$

$x \in=1-1$

$v(k+1)=-1 .+51 * x 1$

$y(k+3)=n$.

75. $K=K+N$

C SIDES NF CLOR.

$D O, 76, K \bar{k}=2, N 1$

$X K=\tilde{K} K=1$

$x=-1:$

DO $77 \quad I=1,2$

DO $78 \quad J=1,2 * N 1+1$

$x J=i=1$

$Y(k+1)=x$

$Y(K+2)=-A+52 * X J$

$Y(x+3)=<3 * x K$

$78 . K=K+N$

$77 x=1$ 
$80 \mathrm{~K} \approx \mathrm{K}+\mathrm{N}$

79. $x=A$

76 CONTTNUE

C

TOD NF CYLNDR.

Do $81 \quad I=1,2 * N 1+1$

$X i=1-1$

DO $81 \mathrm{l}=1,2 * N 1+1$

$x$ J $a: 1-1$

$V(k+1)=-1 .+51+x 1$

$V(\bar{X}+Z)=-A+5 ? * X J$

$V(K+3)=R$

$81 K=K+N$

$N V=K / N$

WRITE (?.82) NV,K

82. FORMAT $(1 / 1 X, 29 H N O$ VECTORS ABOVE $X$ YPLANE, I5,8HSTORAGE = WRITF (2, 51$)(Y(J), J=1, N V * N)$

IF (NV*N IE.NP)GO TO 83

STOD

C FIN UNTT VFCTORS AND TRANSFORM

$8300.00 \quad I=1, N \mathrm{~V}$

$K=N *(1-1)$

SUM $=0$.

nO $91 \quad I=1, N$

$z(j)=Y(i+K)$

O1 SUM $=S U M+Z(J) * Z(J)$

SUM=1. I SORT (SUM)

DO ?? $J=1, N$

$92>(j)=2(i) * S U M$

no os $1 .=1, N$

SUM $=0$.

Dn o\& $J=1, N$

9.4 $S U M=S U M+T(L, J) \oplus Z(J)$

$73 Y(K+1)=S 1 J M$

OO CONTINUE

RETIIRN

50 CONTINUE

51. FOQMAT $(1 \times . O F B .4)$

RFTURN

END.

SEGMENT, LENGTH O99, NAMF SETUNVEC 
SUBROUT INE VMAX1 (N,NV,Y INNV, $X, N X, 11, F F, S M)$.

DIMENSION $B(4), C(4), F(4), F 2(4)$

DIMENSION $Y(N N V), X(N X)$

IF (11.50.1) G0 T0 9

C

$K=-N$

SET $Y$

DO $4 \quad I=9, N V$

$K=K+N$

DO $? J=\bar{M} \cdot N$

$2 R(j)=Y(I+K)$

C

CALI AMATV $(X, N X, B, N)$

CALCULATE LB, STORE IN B

SUM $=0.0$

$\operatorname{BO} ; L=9, N$

$3 \quad S U M=S U M+B(L)+B(L)$

$S U H=S Q R T$ ( $S$ UH)

DO \& JET.M

$4 V(J+K)=\vec{v}(J+K) / S U M$

C

Q EESPRT (FE)

THIS INITIALISES UNIT VECTORS

NOW FIND MAX. VDOT

$S M=-1.0<60$

$K=-N$

$D O F, I=9 . N V$

$K=K+N$

DO $K J=1 \cdot N$

$R(j)=Y(I+K) * F$

$G C(J)=-R(i)$

CAI $C A I E X(N, B, F)$

$C A L C$ CAIFX (N.C.F2)

CALI AMATV $(X, N X, B, N)$

CALI AMATV $(X, N X, F 2, N)$

CALI AMATV $(X, N X, F, N)$

IF STORED IN F

c

s $=0.0$

IB STORED IN B

$52=0$

DO $7 \mathrm{LET}, \mathrm{N}$

$S 2 \nexists S ?-F ?(L) * B(L)$

7 S1 $=(1+F(1) * B(1)$

$s q=\sum \leqslant 9$

s? $20 * 5 \%$

c.

IF(ST-SM) $13,13,0$

CHECK MAX

SMES?

NME $K$

Al 1 a -1 .

13 I $F(S 1-S M) 5,5,0$

$S M=S i$

$N M=\underline{E}$

$A L=1$.

5 CONTINUF

$A L=A I * F$

DO I? $I=1, N$

$12 B(I)=V(I+N M) * A L$

WRI TF(? I 1 1) (R(I),IE1,N),SM,FE

11 FNRMAT (KF12.5)

II $=1$

RFTIIRN

FND 
SUBROUT INE VMAXZ(N,NV,Y,NNV,X,NX,SM,E,IC)

DIMENSION $Y(N N V), X(N X)$

DIMENSION $B(4), F(4), F Y(4), C(4), C 1(4)$

$S M=-1.0 F 60$

$K a \sim N$

กก $4 . I=1, N V$

$K=K+N$

DO $?=T=T, N$

2 $B(j)=Y(i+K)$

C

CAL, AMATV $(X, N X, B, N)$

SUM $=0.0$

DO $\quad Z=T \cdot N$

$3 \quad S \cup M=S U M+B(L) * B(L)$

$S U M=S Q R T$ ( $S U M)$

$A I=F / S U M$

DO $\{\quad J=9, N$

$C(j)=Y(j+K) * A L$

6. $c 1(1)=m(1)$

C

CAII,CAIFX(N,C,F)

CALCULATE LB. STORE IN B

CALI CAIFX $N, C 1, F 9)$

CALI AMATV $(X, N X, F, N)$

CACI AMATV $X, N X, F I, N)$

sian:0

$s ?=0$.

DO 5 LET.N

$S 2=52-B(1)+F 9(1)$

$5 S 1=S 1+F(L) * B(L)$

IF (AMAXT(S1,S2))12,17,0

SHa?

RFTIIRN

$12(5(S)-5 M) 13.13 .0$

$S M=S$ ?

$N M=\bar{K}$

AI $M=-A I$.

13 if $(S-S M) 4,4,0$

$N M=\bar{K}$

$A L M=A L$

$S M=S$ ?

6. CONTINUE

If IC- $-9 i 11,0.0$

no $8 \quad I=9, N$

$8 C C I)=Y(N M+I) * A L M$

WRITF (2.7) $(C(1), I=9, N)$

7 FORMAT $(9 X, 8 H M A X D O T V=, 5 E 12.5)$

11 CONTTNIIE

RFTIIRN

FND

F SEGMENT. LENGTH 376 . NAME VMAX? 
SUBROUTINE SFTA(N,NSB,NSOZ,T,A)

DIMENSION T(NSA2), A(NSO)

$K 3=n$

DO ? I In.NSOS

$2+C I)=0.0$

Dn $4 . K=9 . N$

DO $L$ LET,N

$K T=N S Q * N *(L-1)$

$K 3=x 3+1$

DO $1: 1=1 . N$

$K 2=N S Q+(1-1)+I+(K-1)+N$

$1 . T(K 1+K) i=A(K 3)$

$1 F(1-K) 4,0,4$

KK@

no $31=9 . N$

$00.3 \mathrm{Ja}, \mathrm{N}$

$K K \backsim \tilde{K} K+1$

$K 2=N S Q+(J-1)+1+(K-1) * N$

$3 T(K T+K \geqslant i=T(K 1+K 2)+A(K K)$

4. CONTINIIE

WR T TF $(2.5)(T(1), 1=9, N S O 2)$

5 FORMAT (TX.4EY2.5)

RETIIRN

END:

SUBROUTINE CALE (NX,X,F,M,IV,XM,V,NW)

DIMENSION $X(N X), Y(N W)$

$N \approx V(N W->)$

NVaY(NW;

$E=V(N W=9)$

$N N V=N * N Y$

IECIV-1) $i 2 \cdot 0.2$

IC $C=V(N W-3)$

$C A L I V M A X ?(N, N V, Y, N N V, X, N X, X M, E, I C)$

$2 k=1$

Eax (1)

Dn $1902 . \mathrm{N}$

$K=K+?$

$1 E=F \times(K)$

$E=3: 14959267 / F$

$X N=N$

$E=F * F * X N$

Ea-F

RETIIRN

FND 

